Frauke Mörike

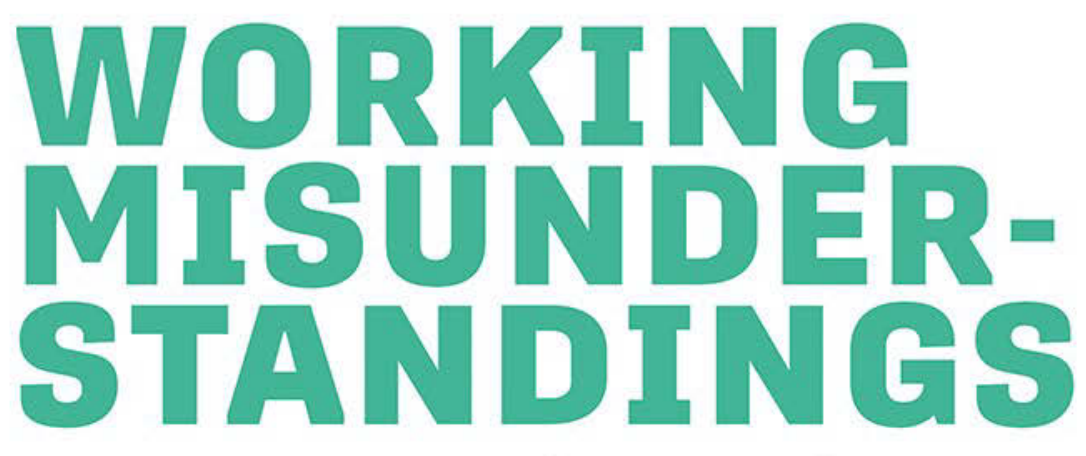

An Ethnography of Project Collaboration in a Multinational Corporation in India

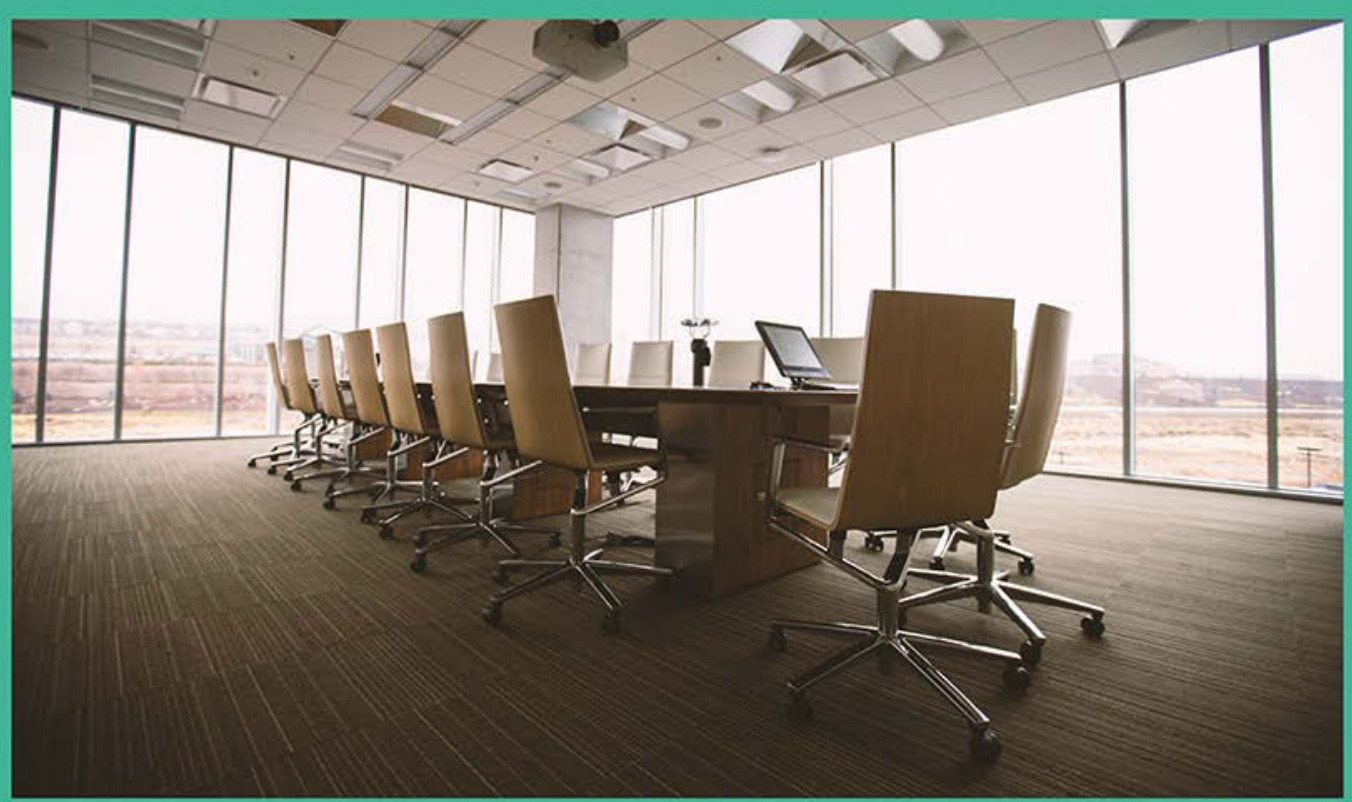

[transcript] LABOR AND ORGANIZATION 
Frauke Mörike

Working Misunderstandings

Labor and Organization | Volume 5 
Frauke Mörike works as a research fellow at the Division of Ergonomics at the Institute of Psychology and Ergonomics of Technische Universität Berlin. She studied business information technology, social anthropology and psychology. Prior to her $\mathrm{PhD}$ in organizational anthropology at the Universität Heidelberg, she worked as an IT-professional in the industry for over a decade. Her research interests focus on Computer Supported Cooperative Work (CSCW) in complex organisations, on assistive technologies in the workplace, and on the development of ethnographic methods for systems design and evaluation. 
Frauke Mörike

\section{Working Misunderstandings}

An Ethnography of Project Collaboration

in a Multinational Corporation in India 
This publication was supported by the Open Access Publication Fund of Technische Universität Berlin

\section{Bibliographic information published by the Deutsche Nationalbibliothek}

The Deutsche Nationalbibliothek lists this publication in the Deutsche Nationalbibliografie; detailed bibliographic data are available in the Internet at http:// dnb.d-nb.de

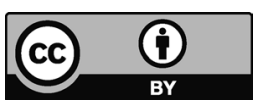

This work is licensed under the Creative Commons Attribution 4.0 (BY) license, which means that the text may be remixed, transformed and built upon and be copied and redistributed in any medium or format even commercially, provided credit is given to the author. For details go to http://creativecommons.org/licenses/by/4.0/

Creative Commons license terms for re-use do not apply to any content (such as graphs, figures, photos, excerpts, etc.) not original to the Open Access publication and further permission may be required from the rights holder. The obligation to research and clear permission lies solely with the party re-using the material.

\section{First published in 2022 by transcript Verlag, Bielefeld (๑) Frauke Mörike}

Cover layout: Maria Arndt, Bielefeld

Cover illustration: https://pixabay.com/

Printed by Majuskel Medienproduktion $\mathrm{GmbH}$, Wetzlar

Print-ISBN 978-3-8376-5867-5

PDF-ISBN 978-3-8394-5867-9

https://doi.org/10.14361/9783839458679

ISSN of series: $2702-7910$

eISSN of series: $2703-0326$

Printed on permanent acid-free text paper. 


\section{Contents}

1. Introduction, or: From IT Projects to Organisational Ethnography .......... 11

1.1. "You should be able to resolve this, right?" ......................... 11

1.2. Office fieldwork in India ....................................... 13

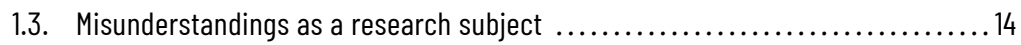

1.4. Organisational ethnography and its limits .......................... 15

1.5. Client centricity and ground reality as opposing values ................. 16

1.6. Chapter outline ........................................... 18

2. Anthropology, Organisational Systems and Misunderstandings ........... 21

2.1. Complex organisations as a field of inquiry .......................... 22

2.2. From organisational culture to social systems ......................... 31

2.3. The organisation as a social system............................ 37

2.4. Conceptualising misunderstanding .............................. 42

2.5. Ethnography as a communication process ....................... 48

3. Fieldwork in Corporate Offices .................................. 51

3.1. Office ethnography: Access and the role of the researcher................. 51

3.2. The fieldwork setting: In and around Advice Company .................. 55

3.3. Methods: Classics with a twist .................................. 64

3.4. Concluding remarks on fieldwork in corporate offices ................. 73

\section{Part I: The Organisation as a Social System}

4. System/Environment Boundaries .............................. 81

4.1. Passing gates: Access procedures ............................. 82

4.2. Differentiated environment:

Clients, freelancers, universities, contractors..................... 96 
4.3. Organisational membership ......................................... 109

4.4. Concluding remarks: Operative closure and openness to the environment ...... 119

5. Internal Differentiation: The Offices .................................. 121

5.1. Increasing differentiation to reduce complexity ....................... 122

5.2. Access procedures: From elaborate to basic .......................... 124

5.3. Inside the offices: Differences in space and equipment.................. 129

5.4. Atmospheres as "tempered spaces": Office perceptions .................. 133

5.5. Concluding remarks: Client centricity as a continuum .................... 150

6. Formal Boundaries, Informal Bridges: Departments and Teams .......... 155

6.1. Differentiating function and hierarchy: Job types and teams................ 155

6.2. Lunchmates and batchmates: Informal bridges across the office ............ 165

6.3. Concluding remarks on the organisational system ....................... 171

\section{Part II: Working Misunderstandings}

7. Working Misunderstandings....................................... 179

7.1. Working misunderstandings and ethnographic insight ...................179

7.2. Working misunderstandings as an analytical category .................. 181

7.3. The client project as a service commodity .......................... 187

8. Collaboration as a Working Misunderstanding ......................... 191

8.1. Discovering "collaboration"...................................... 192

8.2. From a non-intentional to an intentional working misunderstanding........... 195

8.3. Working (with) a misunderstanding............................... 197

8.4. Concluding remarks on collaboration as a working misunderstanding ......... 198

9. Modus intentional: Date games ................................ 201

9.1. Double contingency and cross-system interaction ..................... 202

9.2. Date games and working misunderstandings ......................... 203

9.3. Date games reversed: Status reports and escalation...................... 209

9.4. Date games across system boundaries, and their limits....................215

9.5. Concluding remarks on intentional working misunderstandings ............. 223

10. Modus Non-Intentional: Project Representations ..................... 227

10.1. Organisational decision-making and "black boxes" ..................... 228 
10.2. Lead management: Translating uncertainty ........................ 232

10.3. From strategy to project actions .............................. 237

10.4. The client project as a plan and the "ground reality" ................... 246

10.5. From data to presentations: Project view from "behind the wall" ........... 258

10.6. From presentation files to strategy............................ 270

10.7. Concluding remarks on working misunderstandings ................. 278

11. Conclusion ................................................... 281

11.1. How "Indian" is Advice Company? ................................. 282

11.2. Advice Company as a client-centric social system ...................... 285

11.3. Guiding difference as working misunderstandings ................... 287

11.4. Mutually exclusive values .................................... 289

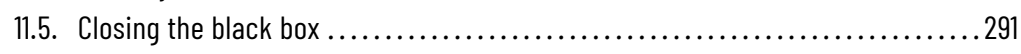

Acknowledgments ........................................ 293

List of Figures........................................... 295

References ................................................ 297 

Dedicated to Matti-

may you keep your interest in opening boxes to find out what's in them. 



\title{
1. Introduction, or: From IT Projects to Organisational Ethnography
}

\author{
You study misunderstandings?! Then \\ you should come to us - there you'll see \\ a lot of them!
}

Whenever I have mentioned the topic of my research, the reactions of my conversation partners have been similar to that of my physiotherapist in the above quote. There has been remarkable similarity across these comments about "misunderstandings", from people of very different organisational settings and work profiles. Whether I have been talking to a childcare teacher, the university's canteen staff, my hairdresser, employees of different business organisations or medical scientists - I have found many to perceive misunderstandings as ubiquitous concomitants of interaction in the work context. From this proposition, it might not be surprising that the idea for this research stemmed directly from my own professional background as an IT project manager in a multinational corporation (MNC). The following example occurred years ago during a project I managed for my former employer.

\section{1. "You should be able to resolve this, right?"}

It was a spring evening in April 2011 and I had worked late in the office in order to prepare for a project status meeting the next day. The project was roughly on track, in most of the work streams. Only one was causing me a headache: a tool that had to be redeveloped, as the old one was no longer compatible with the organisation's new technology standard. The development job had been outsourced to our offshore IT service provider, located in Hyderabad (India). From a seemingly smooth start, the situation had recently problematised. 
Initially, the details of what was expected from the tool had been written into the "requirements document" by a member of the local German IT staff and sent to the development sub-team of the Indian programming team in Hyderabad. Several weeks, over 20 emails and more than 10 meeting hours later, there was zero progress to report on that work stream. Four to five colleagues from teams in Germany and India had spent a significant amount of their time and effort on the project. Nevertheless, the requirements document, which was now enhanced with five additional pages of emails and meeting minutes, was still not answering the Hyderabad programmers' questions. Consequently, the first prototypes were far from the needed solution. Escalation of the situation to management levels in both organisations had not eased the situation. The next day, I would have to - once again - report a red light status for this part of the project.

Why had such a situation occurred? Was it because "those Indian programmers simply were not good enough, although I described everything so well and anyhow I should have programmed it myself", as a German team member concluded? Or was it due to "incomplete and undetailed documentation" from the German team, as the Indian team coordinator stated? I thought I had done everything correctly: I had organised the work tasks in line with established communication forms and processes, team responsibilities and numerous standardised documents. Although all parties worked according to these communication routes, there were clearly inter- and intra-organisational boundaries. At lunch, one of my colleagues from the IT team in Germany asked: "Don't you study something on culture?! You should be able to resolve this, right?" Yes, was I indeed studying "something on culture". Whenever I was not chasing colleagues across the globe to complete their work on my project I was sitting in the library at Heidelberg University, writing my master's thesis in social anthropology on bride price in Papua New Guinea. I subliminally agreed with the colleague's notion that some of the issues we were encountering in our project seemed to pertain to mechanisms operating under the popular label of "culture". But I was not able to see how these issues related to the theories I had learned - let alone how I could use them to "resolve" the situation of the tilting IT project. Apart from being in an unreflexive Lebenswelt of deadlines, task lists and budget numbers at the office, I did not know how to apply the rich body of knowledge from anthropological research to the familiar settings around me.

In the end, the project was successfully delivered - only slightly over time and budget, but involving many more hours and much more nerve than I had 
wished to dedicate to it. Such situations left me with the subtle notion that some misunderstanding had occurred that went beyond the actual exchange of emails and documents; but this understanding was impossible for me to label.

\subsection{Office fieldwork in India}

One year later, in the summer of 2012, I discovered the missing link between my remaining questions on (dys)functioning project collaboration and social anthropology: the field of organisational anthropology. I was electrified to read about the ethnographies of companies similar to my own, involving an analysis of issues I could strongly relate to from personal experience. Two previously very distant worlds with no overlap suddenly appeared mutually interlocked, as I realised that corporate offices could be a fieldwork site for anthropological research. Within a few weeks I decided to embark on a dissertation project in the field, motivated to gain insight into the functioning of the type of organisation I had been working in for more than a decade.

Remembering the project problem from the previous year, I decided to attempt to gain access to an MNC in a typical offshore location, such as India, Malaysia or the Philippines. I did not aim to "resolve" the sorts of critical project situations I had experienced, as my IT colleague had suggested. Rather, I was much more driven by a deep-rooted curiosity about views on the corporate world from a different perspective. For this, I sought a field that was unmarked by my previous work entanglements and former colleagues. Therefore, I refrained from considering research within an office location of my former employer. For the same reason, I also eliminated IT offshore partners and software supplier organisations I had worked with in the past. This left me with an almost blank sheet of options and hard work ahead of me to find an organisation for my project.

In Chapter 2 (Section 2.1), I will outline the various strategies I employed to gain access to an organisation for fieldwork - a task that proved extremely difficult. In this process, I experienced what many anthropologists have written about: gaining access to a business organisation as a research site is much more an organisation's choice than the researcher's (Krause-Jensen 2013: 45). Ultimately, a mixture of persistence, daring and luck resulted in a research opportunity at an MNC in a major Indian city, which will remain anonymous. "Advice Company", as I call the organisation in this book, is a Western- 
origin MNC in the professional service industry and was exactly what I had hoped for: an organisation akin to, but not congruent to, my former company. I was primarily interested in different perspectives on organisational functioning and did not specifically aim for a fieldwork location in India but in any non-western offshore location. Consequently, this work does not attempt to address and analyse Advice Company's traits of "Indian-ness" (Khandelwal 2009). Nor does it seek to map cultural differences between the Indian employees and their counterparts in organisations across the world, as works by other scholars have done (Gupte and Müller-Gupte 2010, Mahadevan 2009, Mayer-Ahuja 2011b). I deliberately chose a theoretical framework and research questions that would allow me to focus on Advice Company's specific organisational structure and internal differentiation. The outcome is a picture or in anthropological terms, an ethnography - of Advice Company, from its employees' perspective.

\subsection{Misunderstandings as a research subject}

Misunderstandings are commonly viewed as inadvertently emerging phenomena that should be avoided. My work, however, will show that Advice Company's working practice relies on misunderstandings as a basic component of communication. I will furthermore illustrate that misunderstandings are used to shape and reinforce mechanisms of power or status in the organisation. Both of these aspects of misunderstandings are relevant for the maintenance of the organisational system, and serve to fortify organisational structures. This work will therefore demonstrate the productive element of misunderstandings and argue that they are necessary for organisational functioning. Drawing on this notion of working misunderstandings - a specific type of misunderstanding characterised by the potential for "parallel encoding" (Sahlins 1982: 82) of a given term or situation - I will address two main research questions:

How do working misunderstandings shape the organisational system?

Why are they productive and necessary for the system's organisation?

This work will build on existing scholarship in anthropology and closely related disciplines, such as Niklas Luhmann's Systems Theory, which argues that 
social systems consist of communication to create and maintain themselves by enforcing their borders with the outside world (see Chapter 2, Section 2.3.3). Misunderstandings are, according to Luhmann, an integral aspect of communication chains. This sociological theory serves as the core theoretical framework under which I will scrutinise the organisation and demonstrate the way in which communication and misunderstandings shape its structure.

This work will contribute to anthropological theory, as Luhmann's Systems Theory has not been adopted broadly in this field (for exceptions see Gershon 2005, Krasberg 1998, Sprenger 2016, Sprenger 2017), even though it is highly suitable for analysing organisations. While Systems Theory has been widely used in organisational sociology and organisational studies, my analysis will demonstrate its further applicability for anthropology. The ethnographic analysis will combine Luhmann's theory with concepts from philosophy (Gernot Böhme's new phenomenology - see Chapter 4, Section 4.4), Louis Dumont's Theory of inverted hierarchy (Dumont 1980 [1966]; Dumont 2013) and the theory of circulating references and translation chains (Latour 2000; see Chapter 10).

More generally, this dissertation will provide new insight into our understanding of misunderstandings in an organisational context: by positioning working misunderstandings at the centre of my project I will add to the field of anthropology of misunderstanding. Furthermore, this office ethnography's focus on misunderstandings in the professional service sector will add to the body of literature in organisational anthropology that aims at understanding organisational functioning.

\subsection{Organisational ethnography and its limits}

This ethnography is the outcome of long-term fieldwork carried out in $2013 / 2014$ at an MNC in the professional service sector in a major Indian city $^{1}$. Advice Company provides advice to clients on strategic decisions. The specific consultancy services the organisation sells is offered by a few global organisations and slightly more locally operating companies. Therefore, I must remain particularly vague about the type of consultancy services offered by Advice Company in order to protect its identity. Similarly, descriptions tion. 
of clients, products and projects will be limited in detail in order to ensure the anonymity of all parties. I have furthermore changed the names of the interlocutors mentioned in this work. By remaining vague about the organisation's background, I am able to provide particularly detailed descriptions of events and my interlocutors' opinions of these events. I have deliberately chosen a personal and self-reflexive writing style with the aim of taking readers with me into this office world - a seemingly all-familiar terrain for most of us.

This work relates events and practices that occurred at Advice Company during the 12 months of fieldwork carried out between February 2013 and June 2014. These events will probably appear to the employees (and maybe by now ex-employees) of Advice Company as accounts of a "very distant past" (Krause-Jensen 2013: 51). The fast-changing organisational system makes the field a "temporal phenomenon", and thus this ethnography provides only a snapshot of a given moment in the organisation's history (Dalsgaard 2013). As Advice Company has an average staff turnover rate of $25 \%$ per year, only a small share of my interlocutors will likely still be members of the organisation when the work is published, and an even smaller number will be likely to hold the same functional positions.

\subsection{Client centricity and ground reality as opposing values}

During my research phase, Advice Company's employees changed teams and offices, or left the organisation as new employees joined. The case studies will illustrate, however, that the organisation's structure and its transactions are not dependent on the individual employees, but on the operations and communication dynamics that are determined by its guiding difference. According to Luhmann, a guiding difference (Leitdifferenz) consists of (at least) two opposing values which steer a system's operations and structure (Luhmann 1995a: 4). At Advice Company, two values are of direct relevance for shaping the organisational structure: "client centricity" and "ground reality". The superior and hence more salient of the two is client centricity, which prioritises closeness to the client as the leading determination for decision-making and working practices.

Advice Company is dependent on a constant flow of project orders from its clients. Consequently, the organisation has established the client at the centre of its dominating value. Knowing what a client wants and delivering a 
project according to the client's expectation form the overarching paradigm that structures the organisation. This might not be too surprising, as client orientation is a well-known management concept, aiming at structuring an organisation and its employees to cater for changing, short-term and differentiated client wishes and expectations (Voswinkel 2005: 11). At Advice Company, this value is established through the connection of internal and external (i.e. client) appreciation - for example, through the display of awards from clients for exceptionally successful projects and a corresponding internal recognition system based on awards for particularly client-centric work. I will show that both organisational structure and internal differentiation are guided by the client centricity paradigm. Furthermore, working misunderstandings and ambiguities relating to the actual meaning of client-centric behaviour serve to maintain these structures.

Client centricity therefore goes beyond a mere principle of efficient organisation, but depicts the primary value according to which the agents align their everyday actions and interactions. This means for example that functions dealing more directly with the client such as client consulting are associated with a higher ranking in the organisation's local value system. Client centricity is salient in management presentations, office talk and during new employee induction trainings with a repetition of rules such as "we never say 'no' to our client". This continuous salience of the value client centricity indicates its overriding importance for the organisational system. In addition, however, the repeated emphasis allows reasoning that the everyday practices are not all running as flawlessly client centric as the organisation's management would like them to run. Based on the principle that if rules have to be accentuated they most likely are not completely adhered to; client centricity has to be repeated frequently because of a competing value undermining it.

This undermining value is not explicitly labelled yet implicitly present in the persistent repetition of the client centricity paradigm. The farther I veered away from the client centric functions in the course of my fieldwork the more pronounced appeared the existence of an opposing value to client centricity. As this value is subordinated, it is less clearly expressed by the agents and hence crystallised only gradually. I have decided to call this opposing value "ground reality" representing all the different manifold aspects of the antonym to client centricity, referring to everything that disturbs the flawless client centric work process. "Ground reality" is a term used in Advice Company referring (amongst other connotations) to those functions and processes most distant to the client. 
Although being a subordinate value located at the lower end of the organisation's ranking, ground reality nevertheless plays a similarly dynamic role in the organisational system and serves to counterbalance the organisation's inclination towards the client. I will show in this work that the two values are mutually exclusive and denote the guiding difference of the organisation.

\subsection{Chapter outline}

The research questions, which focus on working misunderstandings and their relationship to organisational structure, are addressed in the 11 chapters of this book. ${ }^{2}$ Chapter 2 introduces organisational anthropology and complex organisations as a field of enquiry. Following a review of popular approaches to analysing MNCs from the field of intercultural communication, an introduction to Niklas Luhmann's Systems Theory is provided and connected to working misunderstandings as a central element of organisational maintenance. This chapter is intentionally succinct, as more detailed outlines of relevant theoretical frameworks are provided at the beginning of each analytical chapter. The fieldwork at and around Advice Company, together with the methodological approaches taken for data collection, are outlined in Chapter 3.

Chapters 4-10 present the ethnographic analysis and are structured into two consecutive sections in order to open the black box of organisational functioning (Czarniawska 1997: 1): Part I looks at the organisation as a social system and Part II addresses working misunderstandings. Part I illustrates the way in which Advice Company delineates a social system, in the sense of Luhmann's Systems Theory, on the basis of client centricity as a leading marker of relevance, hence the dominant value of the guiding difference (Leitdifferenz). The organisational analysis is developed concentrically, beginning from outside the organisation and moving towards its internal structure. Chapter 4 shows how the organisation establishes its boundaries to the environment and conditions organisational membership. Internal differentiation on a macro-level is discussed in Chapter 5 , which positions the three offices of Advice Company on a continuum ranging from client centricity to ground reality. This differentiation is triangulated via examples of access procedures, 
office equipment and perceptions of atmosphere at the three offices. Chapter 6 traces the organisational differentiation within each of the three offices along a hierarchy of functions (or job types) that corresponds with the two opposing values of the guiding difference. A layer of informal sub-systems is shown to cut across the functional hierarchy of the organisation; in their selfobservation, these sub-systems reinforce the organisational structure along the organisation's reference system. In an interim concluding remark to Part I, Advice Company is positioned as a social system structured along the guiding difference client centricity/ground reality.

Part II builds on Part I's analysis of Advice Company as a client-centric organisation. This second analytical block focuses on working misunderstandings in the organisation that are connected to the client project as a central commodity. It demonstrates how these working misunderstandings shape the organisational system and why they are necessary for its functioning. Chapter 7 commences Part II by introducing working misunderstandings as an analytical category for ethnographic insight, along with a quadrant typology of working misunderstandings. This typology serves as a basic structure for the following chapters, starting in Chapter 8 , which presents a working misunderstanding relating to collaboration that occurred between myself and my interlocutors. Chapter 9 illustrates how "date games" around project timelines contribute intentional working misunderstandings to the planning process across opaque sub-systems, which reinforce the client-centric organisational structure. The hierarchical structure of the values, however, is inverted to favour the ground reality over client centricity during the project execution phase - at least up to a certain point. The client project - as Advice Company's main commodity - is the central topic of Chapter 10. In six steps, the client project is followed through the various departments of the organisation. The analysis begins with a vague project opportunity which might lead to an order and continues along the project development stages to the final delivery to the client. The case studies illustrate that the actual meaning of a project is subject to differing ascriptions along the organisation's project development process. These ascriptions are orientated towards either client centricity or ground reality, and there is constant tension between these opposing values. The organisation manages this tension - or incompatibility - by maintaining the opacity of different project representations as a working misunderstanding. I will show that this working misunderstanding is of central relevance for the communication chain and, hence, the social system. 
The concluding remarks seek to trace the role of the fieldwork country, India, by presenting the reflections of Advice Company's employees and their perceptions of the company as "Indian" or not. The chapter also summarises the various angles taken in the analysis of working misunderstandings and their influence on the organisational system. The answers to the research questions will reveal the rather counterintuitive insight that the successful functioning of an organisation as complex as Advice Company is dependent on the opacity of not only working misunderstandings, but also black boxed organisational processes. 


\section{Anthropology, Organisational Systems and Misunderstandings}

Chapter 2 establishes the theoretical outline by presenting the field of inquiry, the state of research and the main theoretical approaches I depend on for my analysis. I provide a more detailed introduction to the relevant theoretical aspects at the beginning of each analytical chapter (chapters 4-10). Anthropologists' use of organisations as research sites is not new, yet it has recently received an increasing amount of attention. Section 2.1 provides a brief overview of the history of organisational anthropology and contemporary anthropological research in this field. So far, working misunderstandings have not been a focus of anthropological research within organisations, and my work will contribute to filling this lacuna.

The broad potential of the application of organisational anthropology to the business world can be assessed from the rising number of anthropologists working in the industry. With this popularisation has come a risk for oversimplification in response to organisational time and budget limits. Using the example of "interculturalists" (Dahlén 1997), I will show how the tendency to become successful by providing easy answers to complex issues in the fast-paced corporate world has also applied to the popular metaphor of "organisational culture" since the 1980 s. Section 2.2 reviews the most popular theoretical approaches to "organisational culture" within business studies and intercultural management. A critical review of the theories of Geert Hofstede and others provides a first line of thought on why I did not use such approaches as a theoretical framework in my research, but instead adopted the view of Niklas Luhmann's Systems Theory, which understands complex organisations as social systems. An overview of the key elements of Systems Theory is provided in Section 2.3. Section 2.4 illuminates the connection between misunderstanding and systems theory by developing the concept of misunderstanding from that of early hermeneutics to one that sees it as a constitutive element of 
social systems, following the work of Guido Sprenger (2016). A reflection on ethnographic fieldwork as a communication process (Section 2.5) concludes the theoretical sketch that leads to Chapter 3's overview of methodology.

\subsection{Complex organisations as a field of inquiry}

Today's world is an organised world: we live with and within organisations from an early age, when attending childcare and school, joining a sport or chess club and becoming employees of a business corporation or public institution (Garsten and Nyqvist 2013: 1). Organisations have such a dominant position in modern society that even short phases of not belonging to one are regarded as unusual: a year-long world trip requires justification, as does the role of mother or housewife (Kühl 2011: 11). The contradiction that organisations are of central significance in our lives while "knowledge about the actual functioning of formal organisations is successfully blackboxed" (Czarniawska 1997: 1) was one of the motivating factors for my research in this field.

The organisational subject of this work can be characterised as what Christina Garsten and Anette Nyqvist call a "complex organisation" (Garsten and Nyqvist 2013: 12). While acknowledging the potential lack of precision in the term, they confer the adjective "complex" to organisations with high internal differentiation of social positions and roles. Moreover, complex organisations tend not to be defined by their topographic limits: they are much more than their office locality. Garsten and Nyqvist argue that such organisations can be understood more in the sense of Appadurai's "translocalities" (2008), which attempts to capture the interconnections and exchange processes at play between physical places. Garsten and Nyquvist also cite Ulf Hannerz's (2003b) view of complex organisations as "frameworks for flows of people, meanings, ideas and material objects"; this definition stands in close connection to Erikson's concept of "transnational flows", in the context of globalisation (Eriksen 2007: 14). Therefore, complex organisations show interconnected social networks across teams, departments and offices "with formal and informal organisational layers of differentiation" (Garsten and Nyqvist 2013: 12).

This ethnography is an analysis of such a complex organisation, and I will situate it theoretically within the realms of Niklas Luhmann's Systems Theory, which addresses layers of differentiation and complexity (see Section 2.3). To methodologically respond to the translocal nature of complex organisations, 
a multi-sited fieldwork design was employed (see Chapter 3, Section 3.3). This section (2.1) provides an overview of the emergence of organisational anthropology, current research areas and the application of anthropological expertise as a profession in the industry.

\subsubsection{The emergence of organisational anthropology}

Organisations exist everywhere in the modern world, but "at the beginnings of anthropologists' ethnographic research there were few organisations" (Gellner and Hirsch 2001: 3). Although the origins of social anthropology and its key research focus lay in the investigation of ethnic groups in remote, unknown parts of the world, social anthropologists were already part of an interdisciplinary team studying workers at a US manufacturing site in the 1930 s. This project gained major recognition as comprising the "Hawthorne studies", which identified the "Hawthorne effect", according to which employees' motivation and work performance are positively dependent on management attention and individualised treatment rather than physical factors such as brighter light and more breaks (Wright 1994: 6).

Furthermore, the final phase of the Hawthorne studies revealed that the motivational system based on piece rates for an assembly line actually resulted in the opposite of what management had originally intended: the workers did not approximate to the maximum number of pieces they could physically produce in a day in order to increase their earnings. Instead, they had established their own ideas about a "fair day's work", which were considerably below the management's expectation. This study was the first to empirically show a chasm between workers and management - and the existence of an informal organisation (Schwartzman 1993: 13).

Organisational anthropology therefore stood as a counter-movement to Taylorism and other models based on the concept of the homo economicus - the individual driven by rational choice who seeks to maximise a subjective return for all activities (Baba 2006: 85). In this counter-position, organisational anthropology emphasised the existence of an informal organisation by mapping and quantifying interactions between workers that stood in opposition to the formal organisation of corporate management policies and rules (ibid.: 88). Gaining insight into this relationship, the behind-the-scenes politics and other interactions in the organisational "back stage" (Goffman 1959, Stevenson et al. 2003) was quickly seen as a key success factor for business corporations. Therefore, organisational anthropology established itself in business 
consulting in the 1940s and led to a new professional branch for anthropologists outside academia (see Section 2.1.3).

In the 1960s and 1970s, organisational anthropology decreased in popularity until a number of scholars from the US renewed its interest in the 1980s, as reflected in publications such as edited volumes (e.g. Jones et al. 1988) and ethnographic studies focusing on, for example, meetings in organisations (Schwartzman 1989) ${ }^{1}$. Since the 1990s, organisational ethnography has been - especially in the North American sphere - an established subject and management practice tool, and it has been widely taught at US universities (Gamst and Helmers 1991: 37, Cefkin 2010a: 6). In Europe, anthropological institutes (predominantly in Scandinavia; e.g. the University of Copenhagen) offer students an opportunity to focus on organisational research; this has resulted in a range of scholars in the field. A reader with the most prominent texts in the field (Jiménez 2016 [2007]), secondary literature textbooks such as General Business Anthropology (Tian et al. 2010), or the practical guide to methodology by David Silverman (2007) have conversely increased the visibility of anthropological approaches in organisational studies (Ybema et al. 2009: 4).

\subsubsection{Research directions of contemporary organisational anthropology}

In contrast to organisational anthropology as a profession in the industry (see Section 2.1.3), academic base research does not primarily aim at resolving problems that might exist in an organisation. Much more, "the organisational ethnographer is there to map, document, organise, understand, and render a narrative of what was discovered" (Gavin 2015: 99).

The processes, structures and aims of any organisation are heavily dependent on the type of organisation it is. Broadly speaking, organisations can be divided into private business enterprises and corporations, governments, non-governmental organisations, international organisations, armed forces, not-for-profit corporations and universities. On a business area level, corporations can be broken down into manufacturing and service corporations (Tian et al. 2010: 17). The majority of anthropological studies of organisations are conducted on business enterprises, but other organisational types, such 
as trade unions, welfare institutions, civil service offices, universities, hospitals and religious organisations have also been the focus of anthropological research (Gamst and Helmers 1991: 27). Over the past two decades, monograph-length ethnographies have provided perspectives on work in different organisational contexts, ranging from printing machine producers (Orr 1996), management consultants (Stein 2017), innovation agencies (Seitz 2017), to telecommunication industries (Augustynek 2010), restaurant kitchens (Fine 2009), IT firms (Garsten 1994, Wittel 1997, Alvesson 1995), Department Stores (Bachmann 2014), University career career centres (Glauser 2016), NGOs in London (Hopgood 2006) and Zimbabwe (Wels 2003), advertising agencies in Japan (Moeran 2007) and India (Mazzarella 2004), the European Commission (Shore 2000) and contracting and freelance firms at organisational borders (Huber 2012, Barley and Kunda 2004). For a more detailed overview of ethnographic works in organisations, see, for example, Platt and colleagues (2013) or Smith (2001). For an annotated bibliography, see Ybema and colleagues (2009: 260 onwards).

The broad range of monographs shows how various types of organisations play a decisive role in contemporary life. Indeed, areas of study in organisational anthropology are based on the fact that organisations do not exist in a vacuum; rather, they operate in a wider context - an environment - that both provides them with targets and limits their operations. Gender inequalities have long been classic subjects of study in organisations (Hawkins 2008, Ogasawara 1998, Salzinger 2009); for instance, Carla Freeman's work on the female "pink collar" informatics workers of Barbados elaborates on their quasi-professional identity, which is established through their distinctive fashion style and "cool" office look (Freeman 2009).

Turner's notion of "liminality" (1964) has been applied to various contexts of organisational research. While many authors have veered far away from Turner's concept, common applications of the term refer to interlocutors' experiences of ambiguity, unclear roles or organisational structures and the notion of uncertainty at the centre of research. Other aspects of liminality encompass an experience of bonding (comunitas) as a consequence of the liminal status; re-integration into existing or new organisational structures is rarely included in the analysis. Liminality is thus predominantly understood in the organisational setting as a "longitudinal experience of ambiguity and in-between-ness in a changeful context" (Beech 2011: 288). This is the case in Manos Spyriakis' monograph (2016), which explores the meaning of liminal work in contemporary Greece from the perspectives of tobacco and ship- 
building workers, as well as white-collar bank employees. His case studies illustrate that experiences of liminality and liminal status are independent of work type or skill level and encompass diverse forms of individual agency within the economic limits of the actor's choice. Liminality as a state of longterm instability of organisational belonging is also reflected in the white-collar sector by Garsten (1999). Her study positions liminality for highly skilled experts as, on the one hand, a self-chosen lifestyle, and on the other hand, an experience of marginality at the periphery of the organisations they long to join. The latter perception also applies to Advice Company's temporary workers - the contractors - and their desire to make it "on payroll". The work of freelancers in creative industries with liminal work positions is reflected by Tempest and Starkey (2004) and Huber (2012). Similarly, other studies (Czarniawska and Mazza 2003, Sturdy et al. 2006, Borg and Söderlund 2013) have described consultants as occupying a liminal position, as their role as temporary agency workers means they are constantly in a situation of organisational change (Winkler and Mahmood 2015). I will revisit aspects of liminality when analysing the role of temporary contractors at Advice Company (Chapter 4, Section 4.2.4).

"Work culture" as a research field was approached by Wittel (1997) and Krause-Jensen (2013) in the context of ideology, while Augystynek (2010) carved out employees' perspectives on the challenges arising from organisational change and restructuring at the German Telekom. In her study, she traced employees' perceptions of the constant permutation from state-owned institution to private corporation under the laws of economic efficiency and rationalisation. Questions of multi-nationalism are at the centre of Frohnen's study (2005) on the car manufacturer Ford in Germany. Similarly, the relationship of transnational organisations and their managerial practices is reflected in the work of Garsten (1994), who looked at the core-periphery interplay at Apple, and by Røyrvik (2013), who examined a globally operating energy corporation at a moment of crisis.

\subsubsection{Organisational anthropology as an industry profession}

In 2007, Harvard Business Review featured an article titled "The Rise of Corporate Anthropology" (Davenport 2007), in which the author named examples of business corporations that were starting to employ and gain insight from anthropologists' methods of systematic observation. But in fact, the application of anthropological expertise to organisational contexts as an indus- 
try profession started shortly after the Hawthorne studies: Students of W. Lloyd Warner, one of the anthropologists involved in the Hawthorne studies, founded the Society for Applied Anthropology (SfAA) in 1941, which is today the oldest and largest professional association of applied anthropologists, with Human Organisation as the leading journal in the field. Warner himself left the academic setting in 1946 to establish the first anthropologist management consulting firm, and ran a number of large projects on consumer behaviour and both organisational and design anthropology with the leading business corporations of that time (Jordan 2003: 12).

After a decline in interest for anthropologists in business during the 1960 s and 1970s, the tremendous success of Japanese firms in the global economy led Western organisations (in the 1980s) to focus their agendas on understanding the role of culture in the economy and - especially - in business organisations around the world. This development not only led to a rise in scientific publications in this area, but also to the founding of industry-led research institutes using anthropological methodology (Breidenbach and Nyíri 2009: 17). "Culture", which had previously related (in popular usage) to arts, literature and theatre, became a broadly employed buzz word, and the metaphor of "corporate culture" entered everyday language (ibid.: 21-23).

As a consequence, an increasing number of anthropologists began to work outside academia in the business environment, concerning themselves with design, market and consumer research (Sunderland and Denny 2007), organisational development (Diel-Khalil and Götz 1999) and consulting (Cefkin 2010a: 16). Several anthropologists made it into top-level management positions, such as Genevieve Bell, who holds a $\mathrm{PhD}$ in Anthropology from Harvard and headed up the corporate strategy group at Intel before returning to academia. Others became entrepreneurs, such as Jan Chipchase, who founded his own design consulting company after working in top senior positions at Nokia and the design firm Frog. Edited volumes of case studies from industry practitioners provide insight into the applied side of anthropology in business (e.g. Cefkin 2010b, Denny and Sunderland 2014, Pink 2006, Gunn et al. 2013). The EPIC (Ethnographic Praxis in the Industry) conference promotes ethnographic methodology in the industry setting through its yearly conference and active blog ${ }^{2}$.

In 2014, anthropology as a method of insight abounded in popular media: management magazines boasted catchy headlines such as "Stories that De- 
liver Business Insights" (Cayla et. al., MIT Sloan Management Review), "Here's Why Companies Are Desperate to Hire Anthropologists" (Business Insider) and "Applying Anthropology Concepts to Business Models" (Huffington Post). In 2016, the Australian online news source cited the organisational anthropologist Michael Henderson as an expert on "corporate culture" (news.com.au), the German journal managerSeminare (2009) explained the work of organisational anthropologists and anthropologist Andrea Simon wrote in Forbes magazine's online edition about "How Corporate Anthropology can Help Women Drive Change" (2016). Video communities offer a variety of short presentations at TEDx, and other popular conferences are available online, attracting up to 10,000 views. $^{3}$

Such "fast media" (Eriksen 2006: 72) magazine articles and 8- to 15-minute talks from business anthropologists must deal with the challenge of delivering scientific accuracy despite the limitations of the context. Yet their easy to consume, bite-size pieces of information are not only the most accessible accounts of our subject for the wider public, but they are also attuned to the expectations of a business audience for a marketable commodity, which Eriksen claims is lacking in many academic writings (ibid.: 30). In line with this argumentation, Eriksen pled a decade ago for a more visible presence of anthropologists in public debates, stating: "Anthropology should have changed the world, yet the subject is almost invisible in the public sphere outside the academy" (2006: 1). He suggested that we should engage with these "fast media" to require prompt responses to public debates in order to be heard; however, in so doing, we must not forget that "our job partly consists in being speed bumps in the information society, making easy answers to complex questions slightly more difficult to defend" (ibid.: 41).

The work of Julia Bayer (2013) addresses the conflict between journalism and anthropology. She relegates the work and production of journalism by virtue of its need for efficiency and reduction. The journalistic environment is one with which anthropological research struggles and - based on its selfunderstanding - often refuses to connect (ibid.:13). Consequently, while the media presence of anthropologists as consultants allows for an impressive representation of our field in the industry, a number of differences must be taken into account when looking at applied studies of organisational anthropology. The ethnographic work of practitioners in the industry, whether as 
external consultants or researchers inside an organisation, is characterised by the predicaments of a commercial framework requiring proof of direct relevance and applicability of the results. In contrast to academic research projects, projects conducted at, for example, Intel, IBM or Adidas are directed by a narrow focus that is prescribed by the client or stakeholder, with a clear aim and executable results in connection with the expectation of a return on investment for the organisation (Cefkin 2010a: 9).

Similar to the glossy TEDx talks, the results of ethnographic research services are seen as "deliverables" - the central commodity in the service sector. This term characterises successful research as producing clearly refined results at the end of the research and knowledge production process. Such "deliverables" may vary in format from video clips or presentation to standardised project reports, and they often require a compact overview of findings and recommendations for improvement, mitigation or maintenance of a particular situation. The ability to generate such recommendations is generally a core competency for consultants and a requirement that falls very far from that of scientific research. Furthermore, time pressure and budget constraints often result in shorter periods of data collection and analysis. Hence, one of the leading paradigms of anthropological fieldwork, the immersion of the researcher into the Lebenswelt of his or her interlocutors, is barely achievable. This obviously limits complexity while increasing the risk of generalisation.

Insights from such projects must be viewed with all of the above differences in mind. Taking this into consideration - and withstanding the tendency for "academic elitism" (Eriksen 2006: 28) - accounts from office hallways, meeting rooms, production lines and computer screens can provide optimism about the potential for organisational anthropology within business (Mörike and Spülbeck 2019). Reports from peers in the applied industry world give insight into new working practices such as agile software development (Hanson 2014), the practical application of ethnographic methods in consumer research (Valtonen et al. 2010, Barab et al. 2004, Sunderland and Denny 2007) and human-computer interaction (Williams and Irani 2010, Baskerville and Myers 2015; Mörike 2019). Furthermore, examples of anthropologists drawing the line and quitting their industry jobs (e.g. Kitner 2014) can help to advance important ethical reflections in both academic and business contexts. Last but not least, the experiences of those "out there" who need to explain anthropology and the ethnographic method in a few appealing sentences when pitching for a project can be helpful for advocating anthropology (e.g. Jordan and Dalal 2006). 


\subsubsection{Multinational organisations in India as a regional focus}

Offshoring and offshore outsourcing - the process of hiring an external service provider organisation in a foreign (mostly low-wage) country - have been major drivers of the Indian IT industry since the late 1990s (Upadhya and Vasavi 2008: 10). ${ }^{4}$ Several ethnographic studies have been published in this business context; IT and software engineers in both Indian and foreign MNCs in offshore working relationships with their European colleagues (Upadhya 2016). Eaton's thesis (2011) focuses on the collaboration of virtual teams in an IT offshore outsourcing situation between India and the US. While classified as ethnographic work, Eaton's methodological focus lays on virtual ethnography, drawing on interviews and short periods of on-site fieldwork in India. Another work that comprises shorter periods of on-site ethnographic fieldwork in an Indian office environment discusses different perspectives on work across Indian, German and Austrian IT engineers (Mahadevan 2009). The limit of homogenisation in the IT industry across Germany and India is the topic of an interview-based study within the realms of the sociology of work (Mayer-Ahuja 2011a). The edited volume In an Outpost of the Global Economy (Upadhya and Vasavi 2008) garners a range of sociological and ethnographic articles on gender, identity, power and social class in the context of high-technology employment.

A remarkably different view of the Indian IT services industry was taken by Biao (2007), who followed Indian IT professionals who were placed on project-based labour contracts around the world through a practice known as "body shopping" (ibid.: 3). A short auto-ethnographic account of the experiences of a sales representative at an Indian pharmaceutical company sheds light on negotiation practices with clients and organisational structure, though the theoretical insights remain unclear (Banerjee et al. 2011). The monograph Shovelling Smoke (Mazzarella 2004) provides an in-depth review of negotiation processes in the advertising industry, predominantly based on interviews and document analysis. Another relevant edited volume is Anthropologists Inside Organisations - South Asian Case Studies (Sridhar 2008), which delineates different perspectives on anthropological engagement in public sector health and education settings. There are several publications within

For a non-scientific yet interesting account of the Indian offshore phenomenon, see Rastogi and Pradhan (2011), who wrote the Infosys company story as senior managers of the organisation. 
the Indian manufacturing industry sector based on research within business enterprises, including relatively recent works focusing on manufacturing plants (Strümpell 2006, Parry 2009, De Neve 2009).

Within the subject of Indian office work and transnational work relationships, several studies have been carried out within other disciplines, such as linguistics (Gupte and Müller-Gupte 2010, Nakar-Wallraff 2010), business studies (Khandelwal 2009, Pereira and Malik 2015) and business information systems (Winkler et al. 2007). Several of these studies have concentrated on the cultural differences between Indian and European (or Western) enterprises, using concepts relating to national culture and the metaphor of "corporate culture" as a set of measurable dimensions (see Section 2.2 for a critical review of such concepts). Other publications within business studies have focused on foreign MNCs in India (Singh 1979, Garg 1992, Murty 1998, Martinussen 1988) and their business (Johri 1983) and labour strategies (Davala 1995). More recent publications have reflected on the inversion of the development - the expansion of Indian corporations into other markets (Nayak 2011), their (economic) dynamics of developing into MNCs (Vedpuriswar 2008) and their strategies of acquiring firms abroad (Rajmanohar 2007).

This literature review and state of the field analysis has shown that this book connects with established scholarly research in the field of organisational anthropology, as it takes as its basis long-term ethnographic fieldwork at an MNC in India. At the same time, this work fills a gap in the body of research within the professional service industry in India, in general, and corporate MNC office settings, in particular. Similarly, the focus on working misunderstandings adds another dimension to the literature, advancing our understanding of organisational functioning.

\subsection{From organisational culture to social systems}

This section reviews theoretical approaches that aim to shed light on organisational functioning and the mechanisms of collaboration in the work context. In connection with the previously mentioned rise in popularity of the term "culture", several theories have sought to relate organisational success with the metaphor of "organisational culture" (Hüsken 2006: ix). As these theories still enjoy high popularity in business studies, psychology and intercultural communication, especially in the context of MNCs, I will discuss them in this review. Other approaches from organisational sociology and intercultural 
communication will also be reviewed, leading to the conclusion that Niklas Luhmann's Systems Theory is best-placed to serve as the central theoretical anchor for my research, given its detachment of the individual from communication.

\subsubsection{The rise of "organisational culture" as a popular term}

In the 1980s, business leaders and the mass media took a sudden interest in anthropological expertise. The term "culture" became popular and a number of bestselling books broadcast the notion that successful businesses must be concerned with culture (Jordan 2003: 16). In fact, several top-selling management books, such as In Search of Excellence (Peters and Waterman 1982), suggested that organisational culture was an influential "soft fact" in an organisation's success.

This trend was partly due to the rise of Japanese corporations, whose economic strength required explanation; thus, culture leapt to the forefront. In this context, it might not be surprising that the concept of "culture" - relating to the metaphor of organisational culture - was only rarely related to any anthropological understanding of the term (Gamst and Helmers 1991). Even more, the studies of organisational culture in the 1980s often contrasted Western organisations with organisations from Japan and other non-Western countries. This initiated an understanding of organisational culture based on national denominations (Ouchi and Wilkins 1985: 458). Concomitant with the rise of "culture" as a popular term, intercultural trainers appeared in the industry with the promise of resolving the issues arising from intercultural contact. Dahlén's ethnographic study Among the Interculturalists (1997) provided vivid insight into this field, as did Hüsken's ethnography (2006), which portrayed the "tribe of experts" in intercultural management in the context of development projects.

\subsubsection{Dimensions and measures: Hofstede, GLOBE and others}

A scholar whose concepts rose to immense popularity during that time - and who is still taught in intercultural training workshops and university courses - is the Dutch psychologist Geert Hofstede. On the basis of 100,000 questionnaires that were filled in by employees of subsidiaries of the multinational IT firm IBM across 64 countries, Hofstede developed a framework of relevant cultural dimensions in the organisational work context. Taking a view 
of organisational culture as an onion, with "values" at the core and "rituals", "heroes" and "symbols" in the surrounding outer rings, Hofstede related it to national culture-based parameters in order to explain and quantify observed differences in practices between the subsidiaries.

In 1980, Hofstede suggested four (which have since increased to six) dimensions with opposing traits, according to which nations are classified along an index scale from 1 (showing the least degree of one trait and the highest degree of the opposing trait) to 120 (showing the inverse relationship). Currently, the model distinguishes six dimensions along dichotomies of individualism-collectivism, masculinity-femininity (task versus person orientation), level of uncertainty avoidance, power distance (strength of social hierarchy), long-term orientation and indulgence versus self-restraint. Hofstede's model, with its easy-to-grasp, dualistic oppositions advocating an understanding of national culture as the "software of the mind", became vastly popular; along with this popularity came a definition of organisational culture as the "collective programming of the mind that distinguishes the members of one organisation from others" (Hofstede et al. 2010: 344). He connected the approach to national cultures with organisational models by focusing on two of his dimensions, uncertainty avoidance and power distance. Through the combination of the dimensions he proposed a quadrant with four stereotypical organisational models. According to his approach, the typical German organisation is coined by a high level of uncertainty avoidance and low power distance. He refers to publications by other scholars when describing the ideal organisational functioning like a "well-oiled machine" in contrast to the metaphor of the organisation as a "family" for Hong Kong (Hofstede 1994: 7).

Hofstede's approach, which assumed culture was an entity with measurable traits and viewed the world as a set of distinct national cultures, was already outdated in anthropological scholarship before his first publication in 1980 (Breidenbach and Nyíri 2009: 275). Accordingly, Hofstede was criticised not only from anthropology, but also from various disciplines for "mistaking passports for cultural categories" (Gjerde 2004: 144), for "never hav[ing] studied culture" (Baskerville 2003), for being "culturally questionable" (Jones 2007), for showing a "perpetuation of cultural ignorance" (Venaik and Brewer 2016) and for being "a triumph of faith - a failure of analysis" (McSweeney 2002). The debate continues with an article with the title "Does Country Equate with Culture? Beyond Geography in the Search for Cultural Boundaries" (Taras et al. 2016) appeared in an international management journal. While this list of challenging accounts could be continued, it will suffice to state that the criti- 
cism of Hofstede's framework primarily took aim at his suggestion of cultural homogeneity and his neglect of internal differentiation. Critical views from anthropology that Hofstede's assumptions were rooted in an idea of culture as an ascertainable entity are now rare, but still present (Frohnen 2005, Breidenbach and Nyíri 2001, Hüsken 2006, Dahlén 1997). My analysis of Advice Company will illustrate that the notion of a national organisational culture with a homogeneous, holistic construct that functions as a "mental programme" (Hofstede et al. 2010) is undermined by an array of dynamic practices and hybrid constructions of identity within the same organisation and across colleagues with the same country written on their passports: India.

Ironically, one of the loudest critiques of Hofstede's dimensional model in the early 1990s came from Fons Trompenaars, a Dutch business consultant who challenged Hofstede for having only collected data from employees of a single corporation (Hampden-Turner and Trompenaars 1997). Trompenaars' competing model was based on questionnaires that had been sent to multiple corporations in 55 countries; corporations were only included in the study if they passed the threshold of 50 returned questionnaires. Based on this data, Trompenaars provided a framework of seven dimensions to describe the "dilemmas" encountered in organisational culture when collaborating across national boundaries. Hofstede challenged Trompenaars' empirical evidence for the dimensions and accused him of being uninterested in scholarship and tuning "his messages to what he thinks the customer likes to hear" (Hofstede 1996). Apart from the unignorable fact that Trompenaar's consultancy firm grew by $40 \%$ annually in the second half of the 1990s (Kleiner 2001), this discourse shows a persistence in the idea of organisational culture as a measurable entity in connection with national origin.

And it persists even today: In 2004, the Global Leadership and Organizational Behavior Effectiveness research programme, commonly referred to as the "GLOBE" study, published the findings of an extensive series of studies with 17,000 managers in 950 organisations (House et al. 2004). The resulting set of nine cultural dimensions is based on Hofstede's model and provides, per dimension, two separate scores for each country: an "as is" score for actual practices and a score for the way respondents claimed things "should be" done, which the researchers labelled as "values" (Chhokar et al. 2008).

The most recent bestselling publication to build on the idea of employing measurable dimensions to grasp the complexities of organisational culture in a multinational context is by Erin Meyer, an American business consultant who teaches at INSEAD business school. At the end of 2014, she published 
her book The Culture Map: Breaking Through the Invisible Boundaries of Global Business, in which she presented eight scales on which individual countries were placed. However, it is very difficult to determine whether the "research" she and her team conducted over the last decade extends beyond the anecdotes and conversations referred to in her book, on her website and in the various articles she has published in popular management magazines.

Like most of the bestselling books in popular management, Meyer's work has a highly entertaining quality featuring extreme examples that reduce a complex argument to a single insight, and behind-the-scenes anecdotes that provide readers with the impression of a detailed view into the management boards of large multinational players. At the same time, books such as the ones of Trompenaars and Meyers provide short-handed recipes for resolution with little more than shallow theoretical insight (Neuberger and Kompa 1987: 12). Furthermore, these books characterise agents in the organisation as carriers of sub-conscious national cultural practices, and they therefore focus on differences in values, assumptions and behaviour (Frohnen 2005: 44). Viewing culture as a static construction, differences in forms of communication are viewed as cultural differences with a large potential for leading to misunderstanding, resulting in economic inefficiencies or severe losses (Moosmüller and Schönhuth 2009: 216). This view creates a market for intercultural training and consulting projects within the realms of corporate culture and intercultural management. For executives, HR managers and organisational development experts, the idea of culture as a controllable, homogeneous and measurable entity is a driver of notions of organisational culture. The partially problematic line of argumentation of these models seems self-evident for anyone who has been anthropologically trained. Yet the overwhelming presence of these approaches in current business studies curricula, management training and scientific publications (Winkler et al. 2007, Steenkamp and Geyskens 2012), also in the context of Indian MNCs (Khandelwal 2009, Pereira and Malik 2015, Sinha and Sinha 1990), has led me to reiterate the criticism here. Individuals from business organisations who are interested in working misunderstandings in the context of MNC offices might expect this book to centre on dimensions and scales of communication behaviour. Instead, they will be presented with very different insight into the black box of organisational functioning.

Alois Moosmüller claims that both intercultural communication and anthropology seek to promote a world in which cultural diversity is respected or even seen as a resource. However, while the anthropologist is satisfied with 
describing the behaviour and concepts of agents experiencing cultural diversity, interculturalists seek to improve these encounters and to educate and eradicate misunderstandings (Moosmüller 2007:38, Dahlén 1997: 15). But misunderstandings are, as I will demonstrate, a productive element of social interaction.

\subsubsection{Towards a communication-based approach to organisations}

The popular concepts of culture that I introduced above reiterate a limiting and inflexible perspective on MNCs, taking an agent-based view of personality that is multiplied in a community. Conversely, I suggest an approach to organisations that relies on a communication-based concept, for which works on diversity from an anthropological perspective provide a helpful starting point: culture is here regarded as the "product of actors' links through communication" (Moosmüller 2009: 14). An individual usurps the culture of the group with whom he or she interacts most frequently and with the highest intensity (i.e. one's company or family). Via communication, implicit rules and constructs of meaning are created that enable social interaction and impose a certain level of obligation. The term "cultural diversity", in Moosmüller's sense, does not demarcate differences between cultures, but describes the specific differences in culture that become apparent when different cultures directly interact (2009: 15). Organisational culture can therefore be seen as a set of communication rules that become apparent when agents change positions within or across organisations. Employees can change their positions and roles without deconstructing these boundaries. Thus, organisational culture is primarily concerned with distinct sets of communication rules and habits that must be learned upon joining a new team or organisation. Along with these new rules and habits come a new set of communication expectations. This view enables us to conceptually exclude the individual from organisational culture and analytically focus on communication. Communication is the central operation of Niklas Luhmann's Systems Theory, which is introduced in the following Section. 


\subsection{The organisation as a social system}

This section introduces the most relevant topics of Niklas Luhmann's (1995a) ${ }^{5}$ Systems Theory, as they apply to this book. Only an overview of the theory is provided here, as more detailed aspects of Luhmann's comprehensive theory will be provided at the beginning of each analysis section in the following chapters.

\subsubsection{Autopoietic social systems of differentiation}

The German sociologist Niklas Luhmann developed systems theory on the basis of the key assumption that systems do not consist of things, persons or objects, but of operations: biological systems live, psychological systems perform cognitive processes and social systems communicate.

A central element of his theory is the concept of "autopoeisis", which was originally developed by the biologists Humberto Maturana and Francisco Varela. Autopoietic systems are able to reproduce from within themselves, just as plants reproduce their own cells with their own cells. According to Luhmann, the basic idea of autopoiesis can be applied to social systems. Economics, education, politics and organisations are, for Luhmann, social systems within a functionally differentiated society. Through communication - the operation through which social systems create themselves autopoietically - these systems differentiate themselves from their respective environments (Luhmann 1995a: 30). For each system, the environment may be different, but it always defines its boundary to the outer world.

Active and constant differentiation from the environment is necessary to ensure the ongoing existence of the social system. Luhmann refers here to Talcott Parsons, who changed the definition of a system from a static understanding of structure to a dynamic relation to the environment that requires "boundary maintenance" (Luhmann 2006b: 38). This active differentiation to the environment is, for Luhmann, akin to the idea that identity is only

5 The most relevant publication for the theoretical basis of my work was published by Luhmann in German (1984). To avoid translation mistakes I refer in this thesis to the English translation of the title by John Bednarz and Dirk Baecker (Luhmann 1995a). Similarly, other direct translations of Luhmann are also based on English publications of him to ensure coherence. 
possible via difference: a system and its environment exist in constant reference to each other. All operations belong to a system and, at the same time, the environment of another system (Luhmann 1995a: 177). Every system must therefore "maintain itself against the overwhelming complexity of its environment" (ibid.: 182) through constant reproduction (i.e. communication). Reproduction through communication, however, does not guarantee successful system persistence and boundary maintenance. The structures along which a social system reproduces itself are part of the autopoietic system and are determined by the system. This self-organisation or self-reference of a social system can therefore be understood as a determination of structures from within that system (Seidl 2005: 24). As a social system is not only determined by its internal structure but also its differentiation to the environment, this relationship is of particular interest.

\subsubsection{Relationship to the environment: Closure and openness}

While communication is the constitutive operation of social systems, no operation can leave or enter a social system: communication, for example, does not occur across different social systems, as operationally, these systems are closed. At the same time, social systems must have contact with their environment, as they do not exist in isolation: „A system can only reproduce itself in an environment. If it were not continually irritated, stimulated or disturbed and faced with changes in the environment, it would after a short time terminate its own operations, cease its autopoiesis." (Luhmann 1988: 335)

This contact is referred to as interactional openness and it is regulated by the system. The operative closure of a social system means that, on the operational level, the system does not receive direct input from the environment and communication does not directly enter the system. Luhmann compares this to the blood-brain barrier. The human brain is not directly connected to the rest of the body and a number of transformation processes must occur before any matter from the body's physical environment has an effect on the brain. Similarly, an impulse from a social system's environment results in a stimulus at its boundary. This irritation might prompt an internal communication process, but not necessarily. Whether the system ignores this impulse or replies with internal communication cannot be influenced from outside the system. Luhmann refers to this as trigger-causality (Auslösekausalität) (Luhmann 2006a: 401). Through trigger-causality, external events are able to provoke an internal operation in the system. However, the communication 
process that is started (if any communication is triggered at all) is determined by the structure of the social system: „[While] internal events or processes are supposedly relevant to the system and can trigger connective action, events or processes in the environment are supposedly irrelevant to the system and can remain unheeded." (Luhmann 1995a: 183)

Environmental stimuli are thus subject to selection criteria that are set by the system according to its self-determined structures. When a stimulus is deemed relevant to the system according to its self-organised guiding difference (Leitdifferenz), internal communication processes are triggered. The guiding difference of a social system structures the selection process of relevant information and reduces complexity. A system distinguishes information from noise on the basis of this guiding difference, which can be understood as a binary code relating to the system's structure. "Guiding difference" thus refers to the difference between a system and its environment (Luhmann 1995a: xix). For social systems, the binary code organising the selection of meaning can determine an impulse useful / not useful without determining what is selected - only that a selection process must occur (ibid.: 32). Other guiding differences Luhmann identifies for a functionally differentiated society include wrong/correct for science, payable/non-payable for the economy and lawful/unlawful for the legal system. As guiding differences vary between social systems, it is difficult for a system to pre-determine the selection processes at play within another system. Luhmann describes this situation as "double contingency" in cross-system interaction (Luhmann 1995a: 111-13) a concept he adopted from Talcott Parsons to describe insecure knowledge about the interpretation and action of an interacting party. In this context, Luhmann's idea of "structural coupling" plays an important role. When a system's structures are adjusted to the structures of another system in its environment, it is structurally coupled to that system. As a consequence, the structure of the system expects or presupposes specific states or changes in the environment, and this expectation allows it to react to important environmental events and not rely to on contingency (Luhmann 1991: 1432).

\subsubsection{Communication}

According to Luhmann, anything involving a minimum of two psychic systems can be regarded as a social system. However, the constitutive element of a social system is the existence of communication, not the participation of human beings. Luhmann defines communication considerably differently 
from conventional understandings, which rest on the metaphor of transmission from a sender to a receiver (Luhmann 1995a: 139). For Luhmann, communication is a three-part selection process of information, an utterance and an understanding, respectively. Each step selects from a range of options and communication is a synthesis of all three selection processes (ibid.: 141).

None of the three processes form communication in isolation: differentiation between a selection of information and an utterance in the understanding process distinguishes communication from the mere notion of others' behaviour. Consequently, not only can information and utterances be selected from a range of multiple possibilities, but understanding can also be selected from a range of possibilities in order to distinguish information from an utterance (Luhmann 1995b: 115). The inclusion of the selection of understanding in the communication process is not only the main distinction of Luhmann's definition relative to other understandings of communication, but this threestage process of synthesis moves the emphasis from a speaker's intended meaning to the selection of understanding by an addressee. This runs contrary to the temporal sequence of events, viewing communication as a phenomenon that "is made possible, so to speak, from behind" (Luhmann 1995a: 143). The selection of understanding, furthermore, is the crucial element of follow-up communication, as an addressee's selection of understanding steers the next communication event, irrespective of the speaker's intended meaning.

The inclusion of the selection of understanding in communication explains how a communication system can be operationally closed: a system produces its components and structure through communication, itself, and "only communication can influence communication" (Luhmann 1995b: 117, own translation). This is of particular relevance, as it emphasises the participation of at least two psychic systems that do not interact directly. The selection of understanding is therefore not dependent on an understanding of the psychic system, but on an understanding that is implied by the followup communication (Nassehi 2005: 182).

Communication is a synthesis of all three selection steps and cannot be attributed to an individual; rather, it "constitutes an emergent property of interaction between many (at least two) psychic systems" (Seidl 2005: 29, emphasis in original). The relationship between a psychic system and a communication system is nevertheless determined by structural coupling: without a psychic system, communication is impossible. 


\subsubsection{Organisational systems}

Luhmann distinguishes between three types of social systems: societies, interaction-based systems (such as university seminars) and organisations. An organisation is therefore a specific form of social system that can be distinguished from other social constructs, such as families, networks and protest movements. Some organisations carry the very word "organisation" directly in their name (UNO, OPEC); others use demarcating words such as "agency" (NSA) and still others do not have a demarcation at all (Microsoft, Volkswagen) (Kühl 2011: 18).

For Luhmann, organisations are social systems that reproduce themselves on the basis of a specific type of communication: decisions. Decisions are not taken by actors but the social system, itself; in Luhmann's terms, an organisation is a "recursive network of decisions" (Luhmann 2006a: 68, own translation). As outlined in Section 2.3.3, communication - as the synthesis of a selection of information, an utterance and understanding - engenders followup communication and is therefore the constitutive operation of social systems. Similarly, organisations maintain their structure by making decisions on the basis of their previous decisions: a decision is an operation based on former organisational decisions, and it delineates the ground for follow-up decisions in the manner of the autopoiesis of a social system (Brandhoff 2009: 320). A decision is marked by the selection from at least two options, which might be contingent at the moment when the decision is made. While the decision might be initially volatile, it becomes a stable entity on which subsequent decisions are made, and hence enables the maintenance of the organisational system. Organisations can therefore be understood as "decision machines" (Nassehi 2005: 185).

According to Luhmann, the three attributes of membership, purpose and hierarchy are key to illustrating the specifics of modern organisations. Organisations can decide on more or less strong conditions of membership, and can determine who belongs to and who is excluded from the organisation on the basis of alignment with these rules. An organisation sets boundaries for its members' operations; members who do not stay within these boundaries are excluded (Luhmann 1964: 64, Kühl 2011: 18).

Organisations have a clear purpose, such as making profit, serving clients or meeting a demand in the community. Furthermore, organisations operate along hierarchical, rather than democratic lines. They can freely decide upon the specifics of these attributes: they can determine their members, purpose, 
hierarchical structure and the roles within it. By regulating membership, they can highly engineer their members' conduct for a relatively long time (Nassehi 2005: 185).

As a consequence of this, the individual is excluded from Luhmann's systems theory. This exclusion is beneficial for the structured analysis of communication in the organisational system of Advice Company, as it enables a focus on the communication, rather than the individuals, in the organisation. Tracing "organisational culture" therefore means focusing on Advice Company's communication processes (Martens 2006: 104). In Luhmann's sense, operations that create an organisation's social system demarcate its boundaries to the environment and allow for internal differentiation through the emergence of sub-systems. I will therefore focus on Advice Company's communication processes, with a particular focus on misunderstandings.

\subsection{Conceptualising misunderstanding}

Adding the prefix "mis" to the word understanding indicates negative meaning such as "wrong" or "deficient". Misunderstanding, however, is neither the direct opposite of understanding nor doubtlessly located in the area of not understanding; furthermore, the precise meaning of misunderstanding is significantly different from its use in everyday language as merely not understanding. In a misunderstanding, a process of understanding takes place on the basis of an input received, but not in the normatively expected direction of the person judging the misunderstanding. Because of a perceived normatively correct possibility of understanding, both misunderstanding and not understanding are failures to understand, and both are commonly thought to be best avoided. But as a misunderstanding delineates an alternative understanding of an utterance or a situation - in contrast to the "right" (i.e. the normatively expected) direction - it provides a highly valuable source of information about the perspectives of the interacting parties. It denotes a boundary zone where "les cultures s'expliquent et se confrontent, se découvrant différentes" (La Cecla 2002: 103) - where cultures explain and confront, and discover their differences.

In this section, I will argue that misunderstandings are indeed very productive and hence represent positive instances of social interaction. For this, I will follow an approach inspired by Sprenger's (2016) model of structured misunderstandings. In line with Sprenger's argumentation, I will also incor- 
porate Gadamer's (Gadamer 2010 [1960]) theory of hermeneutics, in which misunderstandings are a key element in the process of understanding, and I will connect this theory to Luhmann's Systems Theory.

\subsubsection{Hermeneutics and prejudice}

Hermeneutics, or the art of interpretation, was originally limited to sacred texts and the discovery of the exact, objective, "true" meaning of words. Schleiermacher later widened its scope to include a general hermeneutic of understanding that could be applied to all human texts and communication (Roth 2002: 435). He was furthermore the first scholar to locate misunderstanding at the basis of understanding, as misunderstanding is the default situation when individuals do not make an effort: "The more strict practice assumes that misunderstanding results as a matter of course and that understanding must be desired and sought at every point" (Schleiermacher 1998[1838]: 22). Individuals then risk "qualitative misunderstanding of the content, and the misunderstanding of the tone or quantitative misunderstanding" (ibid.: 23). Schleiermacher goes beyond recommending the avoidance of misunderstanding by arguing for the constitutive significance of misunderstanding as a complement to understanding (Roth 2002: 447). In order to overcome a qualitative misunderstanding, a person's Ideenkreis (body of ideas) must be set aside to allow for an understanding of the writer's distinctive character and point of view. Schleiermacher's approach has constituted a revolutionary shift towards subjectivity and the individuality of the mind (Schurz 1995: 21).

For Gadamer, there is neither not-understanding nor ultimate understanding. Rather, we exist in a constant dialectical process of misunderstanding based on our language and history, which form our encompassing frame - the horizon, or Dasein (existence), of our experience, from which we cannot escape (Roberts 1995: 4). A key element in his argumentation is that persons have prejudices towards certain objects and situations. The concept of "prejudice" is strongly negatively connoted in today's language. Following the tradition of modern Enlightenment discourse it is typically considered as something to be avoided. The reason for this lays in its connotation as an ultimate judgement that is immobile, rather than incomplete (Gadamer 2004: 273). According to Gadamer, a prejudice is merely a point of departure for reflection a pre-understanding from which one can start to ask questions and begin to articulate them. According to Gadamer, we must melt the horizons of under- 
standing and acknowledge that any input received will change our horizons and prejudices (Gadamer 2010[1960]: 311). Even when we are unable to accept a certain standpoint or position, our horizon is enhanced and shifted (ibid: 272). The dialectic process of misunderstanding reworks our prejudices and is thus a productive process that moves us towards understanding. Prejudice and misunderstanding, however, delineate a form of distinction between different social systems (Sprenger 2016: 32). In the following, I will discuss how misunderstanding is an integral part of communication in systems theory and therefore central to the maintenance of social systems.

\subsubsection{Systems Theory and misunderstanding}

Communication, in Luhmann's sense, materialises in the synthesis of the three selection processes of information, an utterance and understanding. Each step selects from a range of possible options and cannot occur in isolation. The third step, the selection of understanding, is crucial with regards to misunderstanding. As psychic systems can never be directly connected, the selected understanding of information is only revealed in follow-up communication, which forms further ambiguities and leads to another selection process (Luhmann 1995b: 116). Luhmann's approach is to move away from the sender/receiver transmission model and the intended meaning of the sender and to instead consider the selection of understanding by the addressee as most relevant (see Section 2.3.3). As this understanding is communicated in the follow-up communication, Luhmann includes misunderstanding as a likely element in communication. He classifies misunderstandings as "controllable and correctionable" (ibid.: 141), as the follow-up communication can indicate which understanding of a communication was selected (ibid.: 143). This iterative line of action can be related to Gadamer's approach to understanding based on re-working prejudices. According to Luhmann, however, full understanding cannot be reached, as the interacting psychic systems are never directly connected.

Furthermore, when communication occurs across social systems, selection processes are performed differently in each system. This increases the potential for misunderstanding due to different selections of understanding. Sprenger (2016: 30) emphasises misunderstanding in this context as a central function of the differentiation of social systems. Following this line of thought, misunderstandings can be regarded as a communicative symptom of system differentiation. In an analysis of misunderstandings, therefore, the 
boundaries between a social system and its environment can become salient for analysis (La Cecla 2002: 14). However, the hypothesis here is that communication functions not only despite, but also much more due to these misunderstandings, as they engender further communication (Sprenger 2016:31). Systems Theory does not assume that information travels across systems; rather, information at a system's boundaries is thought to be reproduced within the system through the selection of understanding, which, at the same time, reestablishes the boundaries to the environment.

In this context, Sprenger distinguishes between structured and unstructured misunderstandings, whereby unstructured misunderstandings relate to "the imposition of one system's concepts upon the semantics and code of another system" (ibid.: 32). When two systems seem to agree on a set of terms and are able to communicate without becoming aware of the underlying discrepancies over a longer chain of communication, Sprenger speaks of a structured misunderstanding. A structured misunderstanding enables two systems to "understand" each other, even though the structures - or the code steering the selection of understanding and follow-up communication - might be fundamentally different in each system (ibid.: 32). As the communication chain still functions, however, the structured misunderstandings are productive for social interaction.

Even Luhmann, himself, refers in the opening chapter of his fundamental work on social systems to structured misunderstandings. Without further clarification the use of the word "system" in the theoretical discussion between scholars suggests an "illusory precision" that one could "only suppose or infer from the argumentation that the participants have different ideas in mind when they speak of systems" (Luhmann 1995a: 1). At the same time, these misunderstandings can be seen as the necessary bridge between systems, enabling uninterrupted (i.e. successful) interaction and hence making interaction "work".

\subsubsection{Misunderstandings in the social system "organisation"}

Organisations are marked not only by conditioned membership and constant negotiation of their organisational boundaries, but also by a high level of internal differentiation. Internal differentiation refers to the way in which a system builds sub-systems (i.e. different systems and (internal) environments within itself). Each sub-system accepts, for its external communicative processes, the primacy of its own. All other sub-systems belong to its environ- 
ment, and vice versa. Hence, communication frequently involves different systems in the sense of interactional openness, and is therefore subject to different selection structures.

In order to foster autopoiesis, an organisation communicates with both systems in its environment and internally, across its various sub-systems that emerged from the need for internal differentiation. Communication chains are therefore often subject to differing selection processes that arise from different selection codes in each of the (sub-)systems. Consequently, an organisation must rely on structured misunderstandings as a basis for continued communication. The communication chain must remain intact; hence, misunderstandings must work to maintain the organisation.

Structured misunderstandings, in Sprenger's sense (2016), are the conceptual basis of "working misunderstandings" - misunderstandings that retain the continuity of communication chains and hence the social system. The term "working misunderstanding" was coined by anthropologist Paul Bohannan to explain the successful interaction between colonisers and African colonies, as both parties were able to "understand" each other through the selections of understanding of their respective systems (Bohannan 1964). Marshall Sahlins refers to working misunderstandings as terms or situations that allow for "parallel encoding" (Sahlins 1982) and thus enable social interaction without dissonance. Working misunderstandings and their role for ethnographic insight are covered in detail at the beginning of Part II of this book, in Chapter 7.

One of my key assumptions about working misunderstandings is that they might not necessarily only work "accidently", due to a fortunate compatibility of selection processes between two systems' structures. While such nonintentional working misunderstandings are certainly an important element of successful social interaction, the examples will illustrate that misunderstandings are sometimes also deliberately maintained by one or both parties. In these cases, although at least one of the involved parties is aware of the different structures and selection processes at play, the communication chain is retained and dissonances do not become salient. These situations describe a "malentendu bien entendu" - a well understood misunderstanding (La Cecla 2002: 25). Organisational culture can hence be understood as a "framework of meaning, a system of reference that can generate both shared understandings and the working misunderstandings that enable social life to go on" (Batteau 2000: 726). 
Communication, including working misunderstandings, as a constitutive operation in organisations and their sub-systems, therefore plays a central role in organisational functioning. Social interaction not only occurs across organisations and sub-systems, with their different codes for selection processes, but it also occurs virtually. Virtual interaction, as a less complex mode of communication, requires recipients to hold a greater number of assumptions, as appearance, attitude and so forth are not on display. Hence, the selection of understanding is more dependent on internal system structures. In virtual interaction, only a few sentences in an email or a brief chat message may be sent. Such messages may be kept short to save time (as they may assume that all parties have a high knowledge of the relevant context and/or historical background (in Gadamer's sense)), but they are often so numerous that they create high communication density (see Chapter 5, Section 5.4.1).

The ethnographic examples will illustrate that Advice Company's functioning is dependent on communication across its sub-systems, which each have their own selection of understanding; such communication can only be realised through working misunderstandings. The social system is maintained on the basis of working misunderstandings, and participating parties reproduce them afresh with each communication between them (see Chapter 10). The case studies further support the hypothesis that such situations also occur in interactions (i.e. communication) between the organisational subsystems and with other organisations. In these situations, working misunderstandings play a major role in retaining the continuity of the communication chains and hence the structure of the social system. In this respect, there is, a "triple entendre" in the title of this book, as "Working Misunderstandings" refers to the theoretical concept of parallel encoding, its relevance for the maintenance of the organisational system and the agents at Advice Company, who are faced with and employ misunderstandings in their daily office interactions.

As Luhmann eradicates the person from his analytical framework, his theory raises reservations - or at least ambiguity (Scheffer 2010: 141) - with respect to its applicability to anthropological research (Lee 2007: 457). Yet his viewpoint is very useful for analysing social networks that the agents also perceive as systems, such as bureaucracies, institutions and other organisations (Gershon 2005: 100). 


\subsection{Ethnography as a communication process}

Given the theoretical presupposition of systems theory, taking communication as a synthesis of selecting information, an utterance and understanding, ethnographic research can also be critically regarded from that perspective. I would like to position my research in a communication process in Luhmann's sense, structured by various selections by different systems. First, this view enables self-reflection on my selection processes; second, it highlights the potential limitations of the insights gained; and third, it allows this work to be understood as part of an ongoing communication process.

As I outlined in Section 2.3.2, the guiding difference of each system determines whether a communicative event is regarded as information or noise. In the context of research, the guiding difference relates to the research questions or topic, which reduce the complexity of the observed system(s) (Keiding 2010: 57). The focus of this research project was the role of misunderstanding in organisational functioning. Carrying out research with my chosen guiding difference of "misunderstanding" most likely resulted in a different set of data than a focus on constructions of gender or food and consumption habits would have. This decision to focus on a specific area of interest inevitably led to a pre-selection of events from the continuous stream of communication at the office, leaving a wide range of events outside my attention. Furthermore, my interlocutors selected information and utterances in relation to the organisational context, but also in relation to my role as a researcher. During lunch, for example, my interlocutors would sometimes switch to Bengali, Marathi or other local languages that I was unable to understand. This selection occurred under the assumption that the communication would be marked as private and hence not interesting for my research. Just as my interlocutors selected information and utterances, I selected an understanding of the information. Depending on the situation - for example, in crisis meetings, phases when my interlocutors had a high workload and when the open plan office suggested that there were too many interested listeners - it was not always possible for me to engage in follow-up communication to clarify my understanding.

Working misunderstandings, on the basis of structured misunderstandings, could occur in the interactions between different sub-systems at Advice Company or between Advice Company and its clients. They could also occur in interactions between myself and the interlocutors. Chapter 7 deals with such a working misunderstanding - drawing on the concept of "collaboration" between me and my interlocutors. My selection of understanding and follow- 
up communications initially "worked" without creating any dissonance in the interaction. But when I changed to a different team during fieldwork, my differing concept of "collaboration" became apparent. While this working misunderstanding came to a point of unravelling, there might have been many other working misunderstandings that remained undetected. This delineates a limitation of research on complex organisations.

This work should therefore be understood as a product of numerous communication chains, with my selection of understanding steering the structure of the following 10 chapters. Consequently, I see this ethnography as my selection of information and utterances relating to the black box of organisational functioning at Advice Company, aiming at stimulating follow-up communication to continue the dialogue. 



\section{Fieldwork in Corporate Offices}

The work environment is, perhaps, the next wave of contemporary ethnographic field research.

(Gavin 2015: 95)

\subsection{Office ethnography: Access and the role of the researcher}

\subsubsection{Why ethnographic research in offices?}

When considering methodology for qualitative research, there are two general options for data collection. The first is to speak with agents about their practices and to analyse the corresponding documents. The second is participant observation over an extensive period of time, with the researcher present on site with access to situational practices and local knowledge(Lueders, 2000 \#6: 384; Latour, 2002 \#37). Extended periods of fieldwork generate results with a depth that is impossible to achieve in less time (Jordan and Dalal 2006: 362). The duration of fieldwork plays a major role in validating this method (see Section 3.2 for a discussion of the research situation); one of the main reasons for this is the involvement of the researcher. Long-term fieldwork demands high research competency, as the main instrument of research is the researcher, herself. It is in the anthropologist's interest to develop an insider's perspective, and for this, she must have exposure to situational orders and practices in order to live with and adapt to them and become immersed in the field (Lueders 2000: 91).

Several ethnographies show that the social research method of participant observation, which was initially developed to study non-industrial societies, is one of the most useful methods for studying human behaviour in general also in the world of industry (Gellner and Hirsch 2001: 9). As participant observation is a context-related strategy of data collection through pluralistic 
methods, it is particularly suitable for my research area: the fast-changing and diverse setting of an MNC, allowing for participation in and observation of cultural events. Furthermore, in this setting, such observations can be complemented by interviews with employees on their opinions, memories and descriptions. The key prerequisite for such ethnographic research in the office context is, similar to all fieldwork, access to the field and establishment of a role that is accepted by the participants (Lueders 2000: 392).

\subsubsection{Access to the field: Managing the gatekeeper}

Once my decision was made to conduct research in an MNC in India, the Philippines or Malaysia, the next practical challenge was gaining access to a research site (Lueders 2000: 392). As I wanted to be as open-eyed as possible, I refrained both from researching the organisation I had worked in for the previous decade and from requesting any funds from the organisation I sought to conduct fieldwork in. Instead, I reached out to all of my contacts in other organisations in that region. However, about 80 emails and 20 phone calls later I was no closer to finding an interested organisation. My contacts were primarily in the business departments of the organisations, and these departments did not have the necessary links to the organisational gatekeepers, typically found in HR departments (McDonald 2005: 457). Another action I took was to contact the Frankfurt Economic Development group and join one of their "India meets Frankfurt Business" sessions. There, I had the opportunity to meet the leaders of organisations of Indian origin; but again, these contacts - while proving quite interesting - did not lead to a research placement. Rather, they requested that I "share my insights" once I was finished with my research.

In the end, a totally different strategy with a component of chance proved successful: I scanned interviews with top managers of MNCs in each of the three countries on expressions of openness to new approaches to maintaining and improving the quality of their employees. When I emailed the chief executive officer (CEO) of Advice Company in India and related my research project to an interview he had given, I received a positive response within 24 hours and was given the opportunity to explain my project in a brief phone call to his HR director. Shortly thereafter, I received a tentative email confirmation that I could conduct my "study internship", as the director liked to call it, at their offices in a major Indian city. 
Having approval from the leader of the organisation proved key to passing through most gates during my research. While gatekeeper management was initially a practical issue, the analysis of gatekeeping structures proved highly valuable for learning about the processes and functions in that organisation (Morrill et al. 1999: 53). The official approval from the uppermost level of Advice Company in India was extremely helpful during the fieldwork, especially when I wanted to gain access to a new department or office location. Poorva from the HR team, for example, once commented on our way to lunch that she found my project great, yet slightly unusual. But she concluded: "You came upon the CEOs recommendation, so there are no questions asked." At the same time, my mode of entry into the organisation via the top was a circumstance that had to be carefully managed during the constant negotiation of my role during fieldwork (Lueders 2000: 392).

\subsubsection{Field entry and role negotiation}

Four months prior to my intended fieldwork start date I planned a three-week pilot field trip to the main office to gain an impression of the feasibility of my research project. I wrote to the HR director about the pilot study idea and potential dates in a detailed, carefully structured email spanning multiple pages. His almost instantaneous response was: "Yes, pls do come. Looking forward." On the basis of these two sentences I booked my flight and stood slightly nervous on a Monday morning at the sleek marble reception of Advice Company, located in one of the many compounds of offices and production sites that represent India's increasing commercial centres (Maitra 2008: 263). After 30 minutes, two people from the HR department, with whom I had never been in touch, picked me up from the reception. One of them greeted me with the words: "Nice to meet you! So you are the one who always writes these looong emails". I was given a visitor's card and we passed the large entrance into the office area. I was asked to explain again - "but briefly!" - who I was and what I wanted. I cut my introduction to the following: "I am a PhD student and I would like to accompany people here in the office to learn about misunderstandings." As none of my previous contact partners was present, the legitimisation I had received from the organisation's top management functioned.

I was asked to sit down at an empty desk and wait again. Apparently nothing had been arranged for my arrival. Deepika appeared again two hours later with a printout containing a list of names - the people I would accompany 
over the next weeks, during my pre-study, each for a day. When she briefly explained who they were, I realised with amazement that she had managed to find members of four different departments, located all across the office of 450 employees. I did not know at that moment that Deepika, with her widereaching ties across the organisation and her practical skills of informally manoeuvring in this setting, would be an invaluable source of information and help throughout my fieldwork. At 2.oopm that first day, I was introduced to the first team manager, who then introduced me to his team member - my first accompanying partner.

I realised quickly that I could not rely on any internal communication about me or my project to pour through the organisation, and that there was no time to explain my role in detail. I had an attention window of only a few sentences to give the colleagues I accompanied an impression of why I would be sitting next to him or her for the rest of the day, before they would turn back to their work. So, I memorised a few key words for the introduction to position myself as a $\mathrm{PhD}$ student, with no ties to management interests and corporate strategy targets. Although my introductions via the respective team managers initially suggested a contrary intention, the fact that the project was financially independent from the company proved a major trust-building argument, and I was very often asked about this. At the same time, I tried to overcome the issue that a Western-looking person in that environment is usually an expatriate or a visiting manager from another global office, by carrying a bag, a note pad and pens with the university logo as visible signs of my student status.

Through this pre-study I not only got a first opportunity to refine my research methods before returning for the main fieldwork, but I also gained confidence that I would receive full support from the gatekeepers (the HR department) and would be able to leverage my role as a student researcher. I was even able to establish a few less formal contacts with some of the younger female colleagues in the office, and this proved invaluable in many aspects upon my return.

Although I always introduced myself as a student, I had to make a constant effort during all of the fieldwork phases to scale my role down to one of a junior researcher and to manage expectations from my direct interlocutors and their managers. Some of my interlocutors, for example, would ask me at the end of a day: "How was I? What is your feedback?" I initially felt slightly uncomfortable in these moments and would start to explain my role again; but I quickly learned to view such questions as an opportunity. By responding: "I 
don't know, I can't judge this - you tell me!" the conversations would veer towards perceived deviations from the average workday or how that day had been different from the way in which the employee would have liked to work. I had other instances of being perceived by middle management as a consultant expected to provide "deliverables" with solutions in respect to their team and work issues.

I also had to clarify at several points that I would under no circumstances convey any information about an individual to any other person - not only for obvious ethical reasons but also in order to not be considered a management spy (Bernard 2006: 357). Interestingly, the HR director, himself, raised the potential issue of me being associated with upper management (Reeves 2010: 319f.). He told me at a later phase of the research that he had intentionally not let me be seen too much with him and the managers: "We wanted to make sure you can move as freely and independently as possible." Yet I observed with interest the developing stories and rumours about my access to the organisation. Agents from different parts of Advice Company referred to communication exchanges between the CEO and my academic supervisor, which had never actually occurred.

My role in the field was thus clearly marked as external to the organisational boundaries, with no formal membership to or financial dependence on Advice Company. This position was nevertheless only partially independent, since I had to align myself with the managers of each team that I accompanied in the assigned timeframe. My interlocutors were certainly aware of this fact and most likely shaped their own behaviour in the beginning with care, infused with a degree of uncertainty over how much of management was sitting next to them in those moments. Consequently, when I analysed the data, I had to consider the point of time in the research process in order to incorporate who my interlocutors felt they were speaking to, and the effect this might have had on their comments.

\subsection{The fieldwork setting: In and around Advice Company}

Advice Company is an MNC of Western origin in the professional service sector, with about 30,000 employees worldwide. Professional service firms, also referred to as knowledge intensive firms (Alvesson 1995) or knowledge-based organisations, rely on a professionalised workforce with specialised expertise and skills in order to produce their products. The products offered by profes- 
sional service firms are characterised by low-capital intensity; that is, their production processes operate without significant non-human assets, such as factories or equipment (von Nordenflycht 2010: 162) - a fact that makes communication-centred analysis on the basis of Luhmann's theory even more pertinent. Advice Company offers consultancy, analysis and advice to clients on relevant business decisions. Within India, Advice Company employs almost 3,000 people and has offices in major cities across the country. As I will show in Chapter 5 , there are three office locations in the city I was located at, each with a different role within the organisation. I was allowed to conduct fieldwork in all three locations, and this determined, to a large extent, my research year and the study design as a multi-sited ethnography in the sense of Marcus (1995). In this section (3.2), I will explore how the field was initially classically defined as a spatial setting in a geographically distant location to my area of origin. Through the inclusion of digital and virtual modes of communication, however, it had to be considered much more widely. The different modes of communication were not only relevant for the field set-up, but were also factored into the selection of research methods (see Section 3.3). Furthermore, I argue that "the field", in the corporate setting, is a temporal phenomenon with consequences for knowledge management during fieldwork, and discuss the relevance of seasonality in this environment.

\subsubsection{Constituting the multi-sited field}

The first office I went to was Advice Company's Indian headquarters, where most of the top management, consultants and service departments (such as HR and finance) are located. With 450 employees covering a diversity of departments and roles, the main office was, for me, the most important research site. Consequently, I allocated most of my research time there and also aimed at setting up my accommodation nearby. Thanks to the contacts I had established during the pre-study, I had the opportunity to move into a shared flat with two young Indian ladies who were friends with some of the Advice Company employees. I lived in this flat, which was located in one of the 10 towers of a large housing compound (called "society") within walking distance to the main office, throughout the entire period of fieldwork. Due to the convenient location to the office, several colleagues from Advice Company also lived in the compound. This circumstance allowed for many opportunities for us to walk to or from the office together, and turned my accommodation - as well as the 
adjacent shopping mall, with its multiple restaurants and coffee shops - into another research site.

The second largest office is located in a central area of the city in direct proximity to the city's primary shopping and leisure areas. Approximately 250 employees work in this office, supporting international colleagues located all across the world through an offshore work model. While the city office location is clearly intended for commercial use, the third office, which I call the street office, is of a remarkably different profile. A windowless, two-storey brick house, it caters for 50 employees, each with an allocated desk, and provides a unique temporary interaction space for 150-200 freelancers. Although the offices are vastly different in their set-ups and are perceived as distinct by the employees (see Chapter 5, Section 5.4), all employees contribute to the organisation's project development process, and this defines the formal relationship between them. A key assumption of multi-sited ethnographic research is that translocal relationships are as relevant as those within each office; such studies depend on the discovery and analysis of the "ties, linkages and relationships different to a mere comparison of localities" (Hannerz 2003a: 206).

Figure 1 provides an overview of the fieldwork locations and the research phases during which I predominantly worked in each office. The research design incorporated all three offices and examined the entire sequence of Advice Company's project development process. I used a multi-sited approach on the micro-level by accompanying members of different departments and teams within each office.

Figure 1: Fieldwork phases and locations

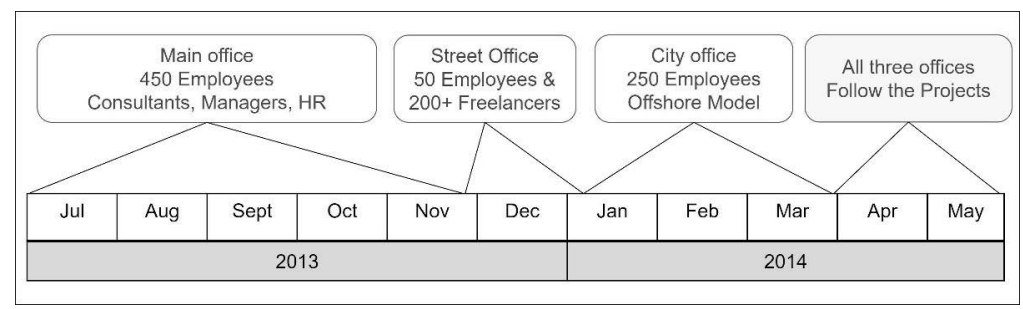

In response to the complex research setting, I employed two of the six strategies of multi-sited ethnography (Marcus 1995: 106): For the majority of my fieldwork, I used the strategy of "following the people", which involved 
tracing the daily work patterns of employees across the office floor, meeting rooms and cafeteria. Accompanying my interlocutors to meetings at other offices or to after-hours activities with their work colleagues (such as wedding receptions, team dinners and weekend coffees), however, added a number of locations across the city to the multi-sited field. During the last phase of my fieldwork, by which point I was familiar with a large number of the employees and business processes, I employed a different strategy in order to change my perspective on the field: I attached myself to three client projects and followed these projects to different agents in various departments and office locations. I accompanied whoever was working on the projects at any given point in order to observe the work steps involved. Although not material objects as such, the projects were the central commodities around which the departments geared up their processes. This second strategy of "following the thing" (Marcus 1995: 107) provided insight into everyday working practices, and this insight was surprisingly different from the insight gained previously. This method can be compared to Bruno Latour's shadowing of a soil sample from its initial capture to its reflection in published findings in a scientific journal (2000). While client projects did not change in status while they circulated through different contexts and departments - as in Appadurai's The Social Life of Things (1988) - the strategy of "following the thing" revealed the previously hidden collaboration strategies involved in their execution (see Chapter 8).

Though I conducted research in each of Advice Company's three offices, which would seem to comprise a clearly circumscribed field, I cannot claim to have gained an ethnographic grasp of the entire field, as multi-sited approaches always require a selection process - in my case, a selection of the departments I would work in and the individuals I would accompany. I was not trying to study the entire culture and social life of these offices, nor of all the employees. Rather, I was trying to get insight into the direct working environment of a number of employees, their daily experiences and their opinions of those experiences (Hannerz 2003a: 208).

\subsubsection{Enhancing the field: Shopping malls, Facebook and other sites}

With the above meta-structure of fieldwork phases in hand and my four key fieldwork sites determined (the three offices and my home), I had to quickly add a number of physical sites to the field as my immersion level rose, such as shopping malls, coffee shops, wedding venues and interlocutors' homes in other "societies", while I accompanied them to out-of-office activities with 
other colleagues. My field mirrored the "enclaved gaze" (Brosius 2010: 66-67) on the city from the perspective of my interlocutors, who would fall within descriptions of "India's middle class" (Lobo and Shah 2015, Baviskar and Ray 2011). My field sites were almost exclusively "new urban spaces", which served as "quasi-colonies, islands or planets of a different kind, only partially connected with their physical environment that lies between them" (Brosius 2010: 69). The descriptions of my travel between these places, such as the trajectory from my accommodation in "society" to the main office, as well as my interlocutors' statements relating to their commuting experiences, illustrate this notion (see Chapter 4, Section 4.1). My field was clearly shaped by my interlocutors and, hence, comprised a restricted selection of places in the city.

Along with my immersion and participation in after-office activities, my involvement in virtual modes of communication also widened my field to include social media platforms such as Facebook, email, WhatsApp and interactive games such as QuizUp. Through this broadening of the research field, I was able to trace relationships and gain visibility into the various channels of virtual communication and bonding used by the employees.

For example, one evening after office hours, I accompanied Sakshi and Raveena to a cake shop with an impressive collection of high-calorie temptations. As soon as we ordered our cake and sat down, the two women took out their smartphones and "checked-in" to the location via Facebook. Then Raveena tagged all three of us to indicate we were at the cake shop together, and posted this with a funny comment that would appear on each of our Facebook pages for everyone in our friends list to see. Reactions such as "likes" or comments were instantly posted by other teammates, and during the next morning's coffee break, our cake session was a topic of further conversation. Similarly, team members would challenge each other to play the mobile phone game QuizUp (a trivia game that requires an opponent) during the day or in the evening, and the result of this game would be subject to cheerful gossip the next day. This field extension was not only useful for tracing informal social relationships (see Chapter 6, Section 6.2), but it also proved a powerful research method for connecting actual events with their representations in the virtual world. The extended multi-sited field hence encompassed a number of physical locations and virtual spaces. My connection to several members of the organisation through social networking platforms and other virtual modes of communication was certainly a great source of insight, as it allowed me to gain a different perspective on interactions between colleagues in the online community. However, this connection not only gave me insight 
into the actors' virtual networks, but it also provided direct access to my own appearance in virtual space, forcing me to engage in "reciprocal transactions of granting each other access to one's virtual social worlds" (Dalsgaard and Nielsen 2013:2).

I would be asked questions about my brother, who had posted a comment on my "pin wall", or where exactly the picture in Singapore that I was tagged in had been taken, and whether it was close to the hotel a person had been to earlier in the year on their vacation; thus, marking also the fact that I was am not the only one transient (Hannerz 2003a: 209). Despite having travelled over 6,000 kilometres, suddenly my "home"1 - even if it was virtual and carefully managed - was part of the field, and my friends and family the subject of casual conversation at the office. A separation of home and work, or my professional and personal self, was not possible - I watched these two domains melt together in front of my eyes on my Facebook page when my "home" friends and my Indian colleagues began to answer each other's comments on a group photo of a wedding reception I had attended with the office crew.

This illustrates the fact that "space", as a primary organising principle of fieldwork, is increasingly challenged and physical distances are more easily overcome through digital modes of communication (Petermann 2010: 120). After my return from India, I remained updated on my interlocutors' afterwork dinners, holidays, weddings and job changes via Facebook and remained in contact with some individuals through occasional chats. I shared online documents containing short sections of the manuscript (generally sections with case studies) with interlocutors who had agreed to look at it and collected their feedback via virtual comments. A year after completing the physical part of the fieldwork, as I like to call this phase, I met one of my interlocutors from the city office for a coffee and interview at Oxford University, where he was about to complete his MBA. One of my flatmates visited Heidelberg during her project assignment in Munich and half a dozen other (ex-)employees of Advice Company moved temporarily or permanently to cities across Europe. Marcus and Fischer refer to this fusion as a "messy, qualitative experience" (1986: 22), yet it constitutes the fieldwork reality (Amit 2000: 8) and represents a classic experience of "study sideways", in the sense of Hannerz (2006:30).

1 "Home" is an unspecific term with an assumed yet subjective quality. In my case, "home" comprised virtual contact with my friends and family via Facebook. For a discussion of the different aspects of "home", see Madden (2010: 45-48). 


\subsubsection{Knowing who doesn't know: Company tenure and knowledge}

I explored above how "the field" - as a spatially demarcated entity - had to be enhanced by virtual components, or, much more, how the agents widened it by giving me access and including me in their various modes of communication. However, especially in organisational contexts, the aspect of temporality should be discussed, as the field must be seen as a temporal phenomenon (Dalsgaard and Nielsen 2013: :2). The structure of the organisation is subject to constant change, with employees joining, leaving and changing departments for personal or career opportunities or due to management-induced major or minor re-organisation.

Within my first weeks of fieldwork, I attended a farewell lunch for a colleague and got an impressive demonstration of the temporal structure of her team when she conversed with a colleague about the different managers they had had. This resulted in a count and the realisation that they had had 10 and 12 managers, respectively, in their two years at the company. The wider discussion at the table then led to a count of the team members who had joined and left the 13-person team in that timeframe and concluded with the stunning number of 27 individuals. When I completed my fieldwork 11 months later, 9 people had changed their seats again. This situation wildly ruptures the "classic image and assumption about the durability of fields and the involvement of 'natives' in them" (Hannerz 2003a: 209), as the length of my tenure in the company equalled and sometimes even exceeded that of the colleagues I accompanied.

When I began my fieldwork I took part in a two-day "new employees welcome" workshop. At this workshop, I was able to meet the 35 people who joined about the same time as I did and who were, much as I, more like "strangers" to the organisation than "natives". However, over the course of my fieldwork - including my stint in the organisation during the pilot study four months prior to the main fieldwork - colleagues began to perceive me as having a certain level of "seniority" in the organisation, or at least a certain "expertise" about it: When I sat with a colleague I was accompanying, Asif from the project coordination team (who I had previously accompanied) passed by, saying: "Hey Nimesh, you don't need to fake a good impression anymore she's here for too long now and has seen everything!" This certainly was not the case, but it significantly eased my trust-building process and helped me to build rapport, and it might have also lowered the all-present observer effect (McDonald 2005: 259). Due to the commonly known fact that I moved 
throughout several teams and offices, I quickly became a source of information for colleagues. I was asked, for example, if I could point out somebody in the database team, whether I knew if it was okay to go ahead with a project despite a final finance signature pending, if I could explain what kind of work the project coordination team did or how "corporate" the main office was. Through these questions, I learned about my interlocutors' different and quite restricted views of and within the organisation, and realised that grasping the emic perspective meant "switching off" parts of my knowledge. Being part of one team meant ignoring certain aspects of the work process that were central to and assumed by another team, and vice versa (see Part II).

Especially when it came to studying the non-intentional and intentional working misunderstandings driven by knowledge concealment between individuals and departments, I found myself in a dilemma. Making use of my full knowledge about certain projects or circumstances and sharing this with the different individuals would have altered the situation significantly, as this information imparity constituted the very structures I was analysing. Hannerz (2003: 210) asserts that with this problematisation of the "local's" and the ethnographer's knowledge, "we have moved away from the classic field work model". Now, the model is not primarily about gaining maximum knowledge, but is much more about knowing what certain individuals do not know relative to others in the organisation.

\subsubsection{Aspects of seasonality and the duration of fieldwork}

There is a lively debate in the discipline on the duration of fieldwork. Previously, exposure to long-term fieldwork of a year or more was taken to indicate research quality and depth of understanding. A number of scholars have challenged this "tacit standard" in our discipline, arguing that fieldwork should instead target the nature of the field and the relevant research questions (Dalsgaard and Nielsen 2013: 4, Hannerz 2003a: 209). Although my total duration of on-site fieldwork added up to one year (including the pre-study), the amount of time spent in the respective offices was aligned with the number of employees, the type of departments and the complexity of the business processes they served, rather than assumed standards of long-term immersion.

The "classic" threshold of about one year for ethnographic fieldwork to generate valid insights was originally motivated by the fact that this duration enables an anthropologist to observe different seasons and to follow the agri- 
cultural food-producing process that (stereo)typically determines the lives of the ethnic groups under analysis. The offices of Advice Company are located in a major city in India; the offices are reasonably equipped and the services offered by the organisation are not dependent on agricultural products. However, seasonality was of relevance to the employees' working life, on various levels.

The weather played a much bigger role than one would expect in a fully air conditioned office environment. Most employees were dependent on public transport and had to commute 60 to 120 minutes from their homes each day. During monsoon season, this meant facing train cancellations and delays, long queues for taxis or rickshaws and endless traffic jams when travelling by bus, private car or motor bike. Constant heavy rains affected even those living in close vicinity to the office, as most were likely to arrive drenched and covered with muddy water from the street. On these days of intense rainfall, people would be challenged to get to the office at all, let alone back home again. Having finally reached the office in the morning, female employees would meet in the ladies' washroom to change their wet dresses and shoes, restore their hairstyles and make-up and exchange stories of who had encountered the longest commute to the office and which parts of the city were completely flooded. Also during the hot and dusty summer months, commuting ladies would meet each morning in the washroom to wash their faces of street dust and city pollution, change their sweaty kurta and style up again.

Religious festivals would bring work-free days and motivated holidays and trips home to the family, as well as after-hours gatherings (and also office gatherings, at desks). Neha, for example, got up from her desk in the late morning and indicated that I should follow her, with the words: "It's Eid, we have to go to Tanika, I'm sure she has brought awesome sevaiyyan" "' Advice Company distributed greeting cards to the employees according to religious group on Diwali, Eid Mubarak and Christmas. These cards were signed by the $\mathrm{CEO}$ and collected and displayed at desks. During the wedding season, many evenings would be spent at teammates' wedding receptions and the next day's coffee talk would be dominated by discussions about the food that had been served. Also from a business perspective, the year was structured by internal and external events. Externally, clients would divide the year into quarterly deliverables and file a larger number of project requests towards the end of 
the calendar year in order to use up their budgets. This would result in higher anticipated and perceived workloads around this time.

As Advice Company's financial year was at the end of March, members of the finance team would push their colleagues in the consulting departments heavily during that month to file invoices and follow up on payments. Advice Company would furthermore structure the year according to a performance review process, wherein the first review would be conducted after six months and a final grade would be given in January/February. The annual performance review, against which bonus payments and promotions would be fixed, would be followed by a wave of people leaving the organisation due to unmet expectations. In contrast, employees showing extraordinary dedication would be recognised through quarterly performance awards, which would be bestowed in a public office ceremony.

These layers of seasonality, which structured the employees' work life, would have been impossible to experience in a shorter period of fieldwork. Conducting the pre-study several months prior to the main fieldwork also proved very important, as it led me to be perceived as having been with the company longer than I actually had been, and ensured a very smooth research start. The fact that I was able to recall situations and events that had happened during the pre-study phase supported my immersion process insofar as I was able to join conversations and stories right at the beginning of the fieldwork. For example, during my pre-study phase, Advice Company organised a party on the roof terrace of the office building. At this party, a magician called three female employees on stage - including me - to assist him with some tricks. Revisiting this experience with laughter with the two other women during morning coffee on the second day of my main fieldwork phase was an invaluable start to the first phase of research.

\subsection{Methods: Classics with a twist}

\subsubsection{Shadowing in the office: Participant/observation?}

Participant observation has been the most well known and widely used fieldwork method in our discipline since Malinowski described it in Argonauts of the Western Pacific (2005 [1921]). The method has the strong potential to capture detailed firsthand knowledge of agents' daily practices and their opinions on these practices, and to provide insight into the often unarticulated 
networks of relationships. In the direct sense of the term, the researcher observes agents' practices while also participating in them. When researching daily practices within complex organisations, the aspect of "participation" is limited insofar as the skills required to take part in the work processes require specialised expertise - commonly referred to as "knowledge work" (Alvesson 1995). As such expertise is not achievable without extensive training, organisational anthropology has adopted the method of shadowing.

Akin to participant observation, shadowing refers to the practice of accompanying a member of the organisation throughout his or her workday. This includes not only sitting next to the person at his or her desk, but also following him or her to spontaneous catch-ups with colleagues and joining meetings, coffee breaks and - in my case - even after-hours outings with team members. Throughout the day, the researcher may ask the occasional question to understand the context of the activity or conversation and to get a direct reaction from the agent on the situation (McDonald 2005: 456). By doing so, the researcher can connect the behaviour or the situation to a comment or opinion on it, and in this way gain deeper insight into the context of the event. Through this method, the organisation can be seen through the viewpoint of the accompanied individual and his or her paths, connections and strategies of working in the organisation (ibid.: 457).

As the general rule of this method is to incur the least possible disturbance of the employee's workday, the researcher must always balance a fine line between asking enough to understand what is going on and to build rapport, and being too "chatty". In Barbara Czarniawska's words, the "point is never to behave like a fly on the wall [...], but to behave like a responsible adult, showing respect and sympathy to others" (Czarniawska 2007: 56). Throughout the day, I would take notes on the agents' activities and conversations, including the small, informal questions I would ask and the employees' emotional states in certain situations throughout the workday.

I shadowed the employees of Advice Company between one and three days per person and changed departments every four to six weeks. But my companionship with each actor was not inactive. Hannerz (2003: 212) discusses this passivity of observation in "settings of modernity", which are difficult to access, and asks what the researcher should "do when 'your people' spend hours alone at a desk, perhaps concentrating on a computer screen". I certainly agree that the corporate context is a setting of modernity. However, it was hardly ever the case that my interlocutors sat isolated at their desks in front of a document and only worked on that task. It was much more common 
to observe a constant flow of conversations on different topics with a broad number of people, both virtually and across the office floor (see Chapter 5, Section 5.4.1).

Even when my interlocutors sat silently in front of a screen and were not involved in conversation (which rarely occurred for periods of more than 20 minutes), they would be exposed to constant input. This input would enter, for example, in the form of emails - announced by a short message popping up in a corner of the computer screen - even though about one-third of the emails would not be directly addressed to the colleague but would only copy him or her in. Furthermore, the organisation's chat programme would constantly inform colleagues of who in their contact list had logged in to the software, with similar pop-up notifications that would disappear after a few seconds. Finally, the general talk of team members in the vicinity of the interlocutor's desk would create a steady flow of information that had to be screened for relevance.

Along with shadowing, I employed several other data collection methods, such as formal interviews. These interviews complemented the seemingly casual conversations that were held during lunch breaks and the feedback collected in observation forms (see Section 3.3.2). Despite the abovementioned limits of participation in this research setting, I was able to switch into a more "participating" format at several points during the day, for example by sharing my dabbah (lunch box) contents with colleagues or by joining after-hours activities such as a coffee with female interlocutors in the evening, a team function at a colleague's home in a suburban setting or a trip with a female colleague to her hometown over the weekend.

During the heavy monsoon phase, I would sometimes physically participate by arriving drenched, swearing to myself under my breath and meeting female colleagues in the ladies' washroom who, like me, had to change their entire outfit. Furthermore, active participation meant the experience of frustration while hunting for feedback and approval from an HR manager on my next department assignment. Participation also involved meeting my neighbour, who was one of the Advice Company managers, for a walk downstairs in our housing compound and accepting the request to play a few rounds of QuizUp from a team member at 11.0opm, just when I wanted to go to sleep. 


\subsubsection{Field notes: Tools and strategies}

One of the most practical issues that I needed to resolve related to the necessity of taking notes on interlocutors who were often on the move (Czarniawska 2007: 57), and lacking a suitable position - let alone the time - to do so. Upon my very first day in the office, I realised that I did not have space to write notes. I sat next to an employee at the corner of their desk and did not want to "invade" their personal work zone. Consequently, the area I considered "my space" was basically the office chair I was sitting on. In this position, it was quite difficult for me to write in my paper note book. Therefore I switched to a small, 7-inch handheld device that I could hold with both hands and type notes into almost like writing a text message. This incidentally resembled the activity of colleagues working on their smartphones and therefore enabled me to blend in more easily with the surrounding behaviour. Most colleagues, in fact, assumed I was writing emails, playing one of the popular online games or browsing the Internet rather than taking notes about my observations. This probably prevented the occurrence of the "embarrassing situation" of my interlocutors "catching" me obviously writing notes about the conversations and events occurring around me (Krause-Jensen 2013: 51). The tool also proved suitable for the office area, as it enabled me to accompany my interlocutors when they spontaneously visited colleagues; I would keep my note pad with me in case these conversations extended to longer discussions. The handheld device featured a simple text application into which I could write my field notes, log and summarise the different interview situations and jot down desk layouts. Furthermore, I used its voice recording programme for formal interviews.

My daily field notes consisted of general descriptions of the setting, the style of the employee's desk, the projects and tasks performed, the outline of the day and - most importantly - the interactions with colleagues. As I accompanied my interlocutors during their average workday at the office (for about 10 hours), I sat next to them at their desk for long periods of time. This gave me an opportunity to note some of their statements - as well as their shorter conversations with colleagues - word-for-word. Over the course of the workday, it frequently occurred that one of my brief casual chats with an interlocutor would intensify into an informal interview lasting 10 to $15 \mathrm{~min}$ utes, before usually being interrupted by a phone call or colleague. Unless the interruption led to another potentially interesting situation, I would use the time to document a paraphrase of our interview directly after it occurred, in- 
cluding a few verbatim quotes; such opportunities are a rare luxury in ethnographic fieldwork. Although these relatively short informal interviews often raised further questions, I would not resume the conversation as soon as the situation allowed. As I had gained permission to conduct my fieldwork under the condition that I would not disturb the employees in their workday, I tried to avoid actively initiating longer conversations. However, at the same time, I did not cut a conversation short if my interlocutors resumed talking about the topic or initiated a new one later in the day.

I created one file of field notes for each day. I wrote predominantly in English, but switched into German for emotional remarks and personal reflections. This was for two reasons: First, I was able to express personal emotional nuances more precisely in German, as it is my native language. Second, the German language in these passages allowed me to quickly identify phases of higher emotional intensity in the data analysis process and to subsequently set the other notes taken during that period into context. For example, such notes could suggest potential biases I might have had at the time of notetaking (Bernard 2006: 392). My note-taking device was pin protected, so my notes were kept safe throughout the fieldwork during the day. Each night, at home, I downloaded the day's jottings from the handheld device to my laptop computer with an encrypted harddrive to compose my consolidated field notes for the day.

\subsubsection{Snapshots and heat maps: How to capture "I'm busy"}

As I touched upon in Section 3.3.1, the employees not only interacted on the basis of direct conversations in the office but also via virtual communication methods such as email or chat, and even within informal virtual channels such as social networks (Facebook) and online games. Therefore, the challenge was not only to conceptualise a multi-sited approach to studying practices within and across physical spaces, but also to manage the integration of "face-toface and digital modes of connection" (Dirksen et al. 2010: 1046). Dirksen and colleagues suggest a solution of "connective ethnography", which goes a step beyond Marcus' method by including the server data of employees' activities on corporate team collaboration sites for social network analysis. Although access to such data was not feasible in my research, I was certainly able to trace and connect social events (e.g. a "cake shop visit") to their virtual representations on social media and other communication channels - or vice versa, to monitor the way in which virtual modes of connection enabled so- 
cial events to be reflected upon and followed up in direct office interactions the following day (e.g. in relation to QuizUp challenges).

However, gaining insight into the connection of face-to-face and virtual communication in work-related situations remained challenging, as I was not included in all email or chat messages exchanged. My interlocutors made statements such as "[The] office is great to coordinate things, but real, concentrated work I prefer to do at home", which suggested that it might be important for me to gain an understanding of the qualities of "real work". Gaining insight into the details of daily work practices therefore meant observing what was actually happening during a "busy" work phase by tracing both physical and virtual communication channels. To achieve this, I adapted observation forms and developed a method I call "activity snapshots", inspired by Wollcott's tracking of a school dean's work day (2003 [1973]).

Throughout their workdays, the employees would interact with each other via various communication channels, often running different active communication channels in parallel and generating a communication density that was experienced as "being busy" and "not getting things done". Through "activity snapshots", this notion could be made visible and could provide a seemingly objective, non-emotional basis for discussion of the topic with my interlocutors. The activity snapshots tracked the flow of information through various channels, such as email, chat, phone (mobile or landline), face-toface discussion and ad-hoc mini-meetings at a desk, over the duration of one hour. For this, I tried to choose an hour when interlocutors were either sitting at their desks or had planned to do so (i.e. when no formal meeting was scheduled). During this hour, I would note in an observation form the frequency with which employees connected with other colleagues and via which communication channels. I aimed at taking snapshots twice per day - once in the morning, once in the afternoon - in order to regularly remind myself of the task and to achieve a broad number of tracking moments. As the employees would sometimes come into the office later or leave early, attend meetings lasting several hours and participate in teleconferences or workshops, this was not always feasible. During tracking, I would ask interlocutors at the beginning of the hour for their perceived work intensity level on a scale from 1 to 10 . Then the distinction would be made between incoming and outgoing connections (i.e. between received and sent emails, received and made calls and conversations initiated by the employee or others). As the employees I accompanied were - in most cases - interested in the results of the snap- 
shot, they were willing to openly share their computer screen with me for that timeframe.

After the hour, I would spend a few minutes with my interlocutors briefly reviewing the data, leaning over my note pad, explaining one or the other situation and checking back with their emails to distinguish "content email" versus "system-generated" alerts, which they didn't read. They would sometimes even point out other contact that had occurred during that timeframe, such as text messages from clients and colleagues. When the situation allowed for it, the activity snapshots were complemented by a more detailed description of the communication pattern and exact timing of each communication. This description gave an impression of interwoven face-to-face and digital modes of connection and the notion of "being busy" in the context of the office atmosphere (see Chapter 5 , Section 5.4.1).

\subsubsection{Tracing informal connections: Webwork diagrams}

While activity snapshots are helpful for collecting data about communication during work, techniques inspired by network analysis (Schnegg and Lang 2002: 7) can help deliver data on whom employees interact with in work-related and non-work-related situations. Especially in Advice Company's spatially defined office environment, it was possible to obtain information about formal and informal relationships through observation. The office comprised a relatively closed environment in which direct interactions could be easily observed and matched with interlocutors' own assessments. I systematically traced agents' activities and asked agents to draw small-scale egocentric network diagrams. Through these observations, my aim was to uncover informal relationships by analysing which agents interacted with each other in relatively "free-choice" situations such as breakfast, lunch and high tea and general questions versus non-choice situations (e.g. those relating to client projects). For this, I distinguished the contact category "Work-related nonchoice" (e.g. project team meetings); "Work-related choice" (e.g. asking for advice or help); and "Non-work related" (e.g. lunch or chai (tea) breaks).This analysis was conducted for each of the colleagues I accompanied, for at least two days. I am aware that the data gathered through observing visible interactions in the office is more accurate than the data gathered on less openly visible contact channels, such as chat programs, except in cases when the agents chose to share their computer screen with me (for example, during collection of the activity snapshot). 
The egocentric network diagrams aimed at uncovering relationships with members of the organisation (irrespective of team) in more detail, and were based on employees' own assertions. These diagrams were built beginning in the second half of the fieldwork phase, and primarily with colleagues I shared good rapport with. This is because I not only asked interlocutors about the colleagues they preferred to interact with, but also about the colleagues they tried to avoid, and why. Through applying this method, I was able to trace the informal network of lunch groups in the city office, as presented in Chapter 6, Section 6.2.

\subsubsection{Interviews: Strategising the record button}

Interviews were my central method of collecting information about the professional background of my interlocutors and they enabled me to understand the wider context of the situations I observed during the day. The interviews were conducted formally (i.e. in situations clearly marked as interviews that were arranged in advance). Furthermore, as described above with respect to the field notes, I employed the shadowing technique for many informal interview situations and created structured documentation of these. While the spontaneous, informal interviews throughout the day provided valuable insights and often concise statements from my interlocutors about situations or interactions, the formal interviews allowed for more concentrated and (mostly) undisturbed in-depth conversations on potentially controversial topics.

Ad-hoc, informal interviews were of tremendous importance in this office environment. My casual questions about the latest timeline discussion while accompanying an interlocutor to the coffee machine, chit-chat about an interlocutor's background situation over lunch, or an interlocutor's upset grumble to me about a heavy workload as we walked to the gate of the office compound provided deeper insight into their opinions of the events I observed as part of their work life. Some employees had three meals per day at the canteen and others took cigarette and chai breaks; all of these moments provided good opportunities for me to gain extra moments of their time, build rapport and ask questions. When informal interviews took place away from my interlocutors' desks, meetings or other places where I could document their comments immediately, I would write the minutes from memory as soon as I could access my handheld, following the rule: "If you don't write it down, it's gone" (Bernard 2006: 389). Yet in these settings, I had to bear in mind that the 
situation was never really informal and that my interlocutors were very aware of my role and my interest in "browsing for information", as an interlocutor put it.

Semi-structured formal interviews were conducted with employees from all levels of Advice Company. I adjusted the interview guide to each situation by adding questions relating to situations I had observed with the interviewee. As the majority of the interviews were held during office hours, I had to target a length of 20 to 30 minutes to ensure my interlocutors would agree to the interview. In order to fit employees' work schedules, the interviews usually took place in the early evening, between 6.00 and $8.00 \mathrm{pm}$. Whenever possible, I held these in a meeting room, which my interlocutors had to book. Once all of the logistics and schedules had been sorted out, the interlocutor and I would sit in one of these meeting rooms - windowless rooms of 5 square metres equipped with a glass sliding door into the office area, a round table and three chairs.

Doubtlessly, the formal setting and the official nature of a recorded interview situation emphasised my role as a researcher collecting data. However, through this set-up I ensured that I had the opportunity to talk to my interlocutors in a relatively secluded, peaceful and confidential environment, even if only for a very limited amount of time. As the meeting rooms were reserved by my interlocutors, they were assigned a formal hosting role and this balanced the potential hierarchical disequilibrium of my position as interviewer. Our situation also resembled the familiar event of a business meeting, in which one talks about a previously agreed topic. As Bernard points out, semi-structured interviews are useful for actors who are used to efficiently using their time (Bernard 2006: 212).

To achieve the greatest possible focus on my interlocutors in the interviews I seated myself at the small table facing the sliding door into the office, so that my interview partners would automatically sit in the opposite chair. With their back towards the office, they would avoid scanning the actions going on in the office during our interview. Placing the handheld device on the table and pushing the red button to record the interview sometimes also helped to prevent interruptions from mobile phone calls, and again stressed the formal nature of the situation. To create a less formal setting, I switched off the recording after having asked around two-thirds of the questions. I then continued to ask the remaining questions in a less formal manner and took note of the answers in bullet points in a small paper note book. This resulted in a significantly more relaxed, literally "off-the-record" atmosphere, giving 
me the opportunity to ask about controversial topics. Often, this technique resulted in a voluntarily prolonged interview.

In other situations, I refrained from recording a formal interview at all. These situations mostly occurred when an interview had to be conducted at a location unsuitable for recording due to loud background noise, such as the street office, the office canteen or a public coffee shop. In some cases, I judged recording as inappropriate as it would place too much emphasis on the formality of the situation; for example, when interviewing an employee about his or her decision to change departments or leave the organisation. In these situations, I took notes during the interview, and transferred these notes into a more detailed memory protocol on my laptop as soon as possible after the interview.

\subsection{Concluding remarks on fieldwork in corporate offices}

This chapter has shown that fieldwork in corporate offices faces similar challenges to research in other settings: gatekeeper management, role definition and an interlocking analogue and virtual world are part and parcel of ethnographic research. However, the corporate office, with its clear structures, requires a correspondingly structured fieldwork approach with a clear plan. Accordingly, the "classic" methods of ethnographic research must be adapted. In the present environment of highly specialised experts of commercial topics, I used shadowing, rather than participant observation, as the primary method of data collection. Getting a share of the actors' time - a highly precious resource at work - required constantly hunting for opportunities while not competing with colleagues. On the other hand, the office provided a very suitable setting for analysing communication channels and observing social networks.

Because I was outside the organisational system, I was able to move freely within each of the three offices and to chat with Advice Company's employees on very different teams and hierarchy levels. Although I was assigned to different teams throughout most of my fieldwork, I enjoyed an almost limitless level of mobility. This allowed me access to conversations with a wide network of people that only long-term managers at Advice Company enjoyed similar access to. At the same time, my external position enabled me to more easily build rapport with my interlocutors and lowered their reluctance to engage in informal conversations with me. 
This position, however, also limited the applicability of a particular research method I had initially planned to employ: focus groups. With the aim of engendering discussion among participants, focus groups are viewed as useful tools for gaining deep understanding of potentially controversial topics. After running three focus groups and attempting another two, I decided to refrain from using the method due to my practical inability to recruit a handful of people at the same time. My attempts were fruitless: whether I planned the focus group meeting days in advance or tried to gather participants spontaneously - neither method worked. Client deliverables, urgent issues or simply rare opportunities for colleagues to knock off early led to situations in which I was sitting on my own in the meeting room. The only ways I achieved some level of presence were either employing a confederate amongst the participants to motivate his or her peers to reluctantly leave their tasks or mentioning to a manager that I wanted to hold a focus group with some of his or her reports. Either way, I had to rely on the organisation's structures to get participants into the meeting room, and this did not lead to the open and motivated discussions I had imagined. On the contrary, my dependence on the organisational hierarchy or advocates in a team endangered the rapport I had built with the interlocutors. My position outside Advice Company's structures was beneficial for the remainder of my research, but clearly not for conducting focus groups. In closer alignment to my role, I therefore used lunch tables, taxi rides and other informal, more or less incidental group moments to instigate discussions of topics I was interested in.

Of all the opportunities and challenges during fieldwork discussed above, the most remarkable was the information asymmetry I encountered between different teams and departments. Assessing and managing the different levels of information provided about a client project required careful management. However, analysis of the different selection processes at play in each of the teams proved a fruitful source of insight into the organisational functioning. 
Part I: The Organisation as a Social System 



\section{The Organisation as a Social System}

For the analysis of complex organisations and misunderstandings Niklas Luhmann's Systems Theory serves as the central theoretic approach and part I of this book analyses Advice Company as a social system. In order to grasp the complexity of the organisation, I adopted a concentric approach and chapter structure.

Figure 2: Chapter outline, Part I

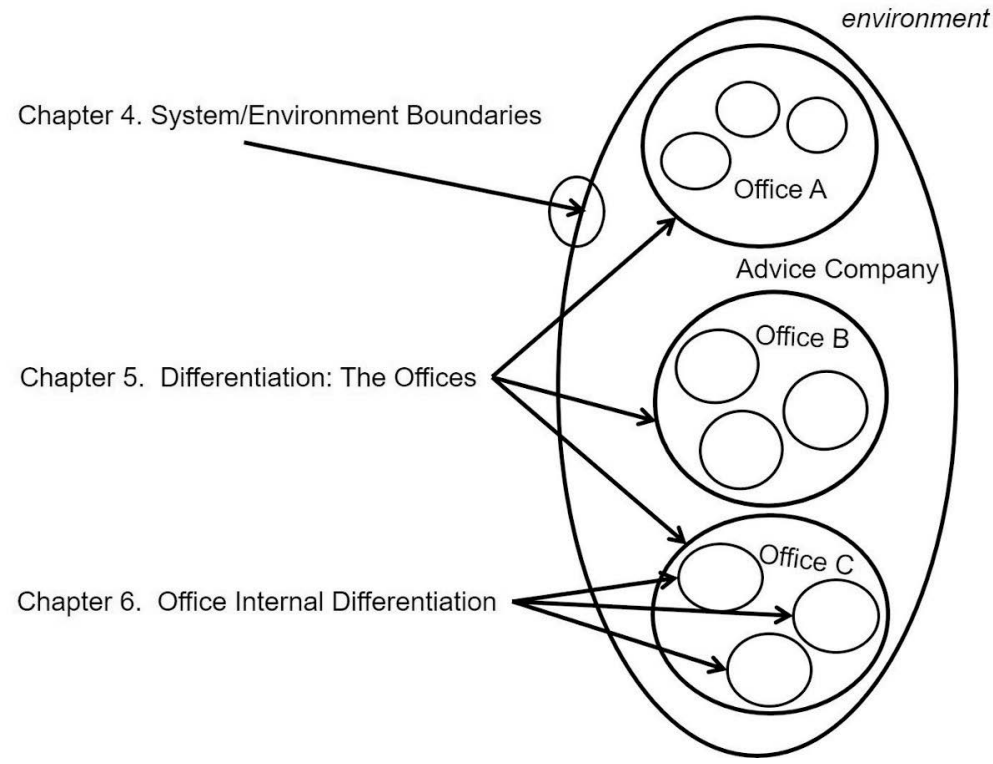

Beginning with an emphasis on the boundaries of the organisation as such, Chapter 4 concentrates on the differentiation of Advice Company from the environment. An overview of daily practises of organisational boundarymaking is followed by an introduction to the relevant systems in Advice Company's environment, before aspects of organisational membership are discussed. The chapter concludes with a reflection on the operational closure and interactional openness of these organisational boundaries. In Chapter 5 , the focus narrows to the internal differentiation of Advice Company. By comparing the access procedures, office equipment and perceived atmosphere of the organisation's three offices, I show that they can be placed on a contin- 
uum orientated on the central organisational value "client centricity" and its opposition, the "ground reality". Chapter 6 provides insight into each individual office and traces the invisible boundaries that cut across the open plan office, as perceived by the employees in their daily lived praxis. The ethnographic data illustrates the way in which these internal boundaries repeat the inter-office differentiation structures, as described in Chapter 5. Within each office, further sub-systems emerge along the value client centricity. In the street office, the incompatibility of client centricity with its opposition, ground reality, with respect to emerging sub-systems, is almost tangible. But this study would not be ethnographic without also shedding light on the informal "bridges" across these boundaries. The informal sub-systems of lunch groups, for example, allow for an even more differentiated view of the organisational structure.

\section{Organisational differentiation and decision-making}

According to Luhmann, systems are processes that are created by their perpetual self-(re)construction (autopoiesis) through a single, specific mode of operation that marks their difference to the environment; in the case of social systems, this mode of operation is communication (Luhmann 1995a: 35-37). The system-environment relationship is constitutive of a system's evolution insofar as identity emerges through differentiation to the environment. But the environment, itself, is highly relevant for the maintenance of the system, as it supplies the necessary resources of energy and information (ibid.: 177). For the following analysis, it is important to note that the system-environment distinction occurs twice: once when the difference is produced by the system through its operations and again when this difference is observed within the system through the process of self-observation (ibid.: 178-80; Luhmann 1997: 45).

According to Luhmann, organisations are a special type of social system with distinct properties. For an organisation to emerge, there must be educational, economic and legal systems to support it: the first supplies adequately skilled resources, the second allows for paid labour and the third enables the enforcement of binding contracts (Luhmann 1997: 828). By observing the access procedures at the reception of Advice Company's main office, I identified the environmental systems that the organisation interacted with, and the relevance of these systems. Every system determines which parts of an 
environment are relevant, and these decisions are manifested in the system's observable communication structures. Organisations are further marked by conditioned membership, with the decision to enter an organisation based on a combination of self and extrinsic selection, which is terminated by the decision to leave the organisation or to be released from it. Through entry into an organisation, a member agrees to comply with the given rules. Failure to do so results in loss of membership. However, this process only functions if membership remains sufficiently attractive; this is usually regulated through salary (ibid.: 830).

The central operation of an organisation is decision-making. Therefore, Luhmann also defines organisations as those social systems that engage in communication processes relating to decisions (ibid.: 833). Organisational decisions most obviously relate to personnel decisions on membership and internal roles, but also to communication channels, with structural implications on reporting lines, work task programmes, vision statements and so forth (Luhmann 2006a: 225). One decision engenders the next one: for example, the decision to hire a member triggers subsequent decisions on who gets assigned to which project. For Luhmann, this chain allows for uncertainty to be absorbed, as a decision always implies at least one alternative. The decision is therefore a volatile construct in the moment it is taken, yet receives affirmation through the decisions that follow it, as the condition under which the initial decision was taken becomes irrelevant (Luhmann 1997: 830). An example of this is provided by Advice Company's decision to provide me with access to the organisation as a field site: Once this decision was taken by the management, subsequent decisions were grounded on this proposition. Consequently, the following decisions did not refer to my presence in the organisation, but instead related to whether I should have access to internal documents (the ultimate decision was that I should not) and with which department I would start. Subsequent decisions neither questioned nor challenged how and why the initial permission had been given but continued with the decision-making. 



\section{System/Environment Boundaries}

Organisations are, similar to all social systems, operationally closed. This means that all operations - in particular decisions and communication exist to reproduce that particular system and no other. Operations cannot leave or enter a system, but organisations can manage interaction with other systems in the environment; they are open, on an interactional level. Such interactions are regulated and controlled by the organisation, mostly through hierarchy (Luhmann 1997: 834). Only specific elements participate in environmental interactions and they also influence the system internally (Luhmann 1995a: 197-98). In Advice Company, such interactions are performed by certain functions that are specifically assigned to have contact with other systems in the environment.

Emil Røyrvik concluded, from his study on corporate managers, that such externally communicative functions are primarily concerned with regulating a company's "boundary conditions" (2013: 156). Self-evidently, not all members of an organisation are involved in such "organisational borderwork" (Faßauer and Geithner 2016: 94), but I will demonstrate in this chapter that, in Advice Company, several functions - situated at different levels of the organisational hierarchy - are concerned with such tasks. The most apparent of these are the client consultants, who manage the interaction with the client systems. However, HR employees also interact with the educational systems and manage the entry of new organisational members.

This chapter analyses my observations and interviews relating to performed and perceived boundaries between the organisation and its environment. Section 4.1 illustrates the way in which Advice Company stresses its structural limits, not only in physical, functional and territorial terms, but also in temporal and linguistic terms, as exhibited by the working practices of its employees. I commence with observations relating to physical access to the organisation and focus on the diverse processes through which differen- 
tiation is produced at the reception of Advice Company's main office. Section 4.2 analyses the organisational environment, focusing on the systems that play a relevant role for Advice Company. Based on the ethnographic material, I draw further inferences about the interacting systems, their distinctive qualities and the way in which these interactions are managed within the organisation. I illustrate this with an example of boundary maintenance in conjunction with decision-making around the topic of organisational membership. I also analyse the processes of entering and leaving Advice Company and the conditions on which employees maintain membership. The chapter concludes with a discussion of the organisation's boundaries with the environment and the structural implications that arise from this set-up.

\subsection{Passing gates: Access procedures}

The main office of Advice Company in the city serves as the organisation's national headquarters. It is located in one of the newly developed business park areas in the city, where several larger national and multi-national offices are grouped in one of India's typical "urban high-tech spaces" (Maitra 2008: 263). The office building is therefore part of a larger office compound that hosts other MNC offices and production sites. Advice Company has approximately 450 employees at that location and, in total, an estimated 15,000 people (including those from other companies) pass through the gates of the business park each day. In the neighbourhood, several other office compounds display the names of other MNCs in the consulting industry. An estimated 100,000 workers travel in and out of the area every day, resulting in a challenging commute, as outlined in Section 4.1.3.

Entrance to the office compound is only possible via a road with heavy traffic. The massive entry gate for cars and buses is patrolled by security guards in uniform, who require each entering person to produce "the badge" - an employee ID card from one of the residing companies - before passing on to the next checkpoint. Pedestrians must pass through two smaller gates, each with two guards, who will not let them pass without "the badge". For persons who cannot produce such a badge, a small booth with a hectic queue in front of it issues visitor passes each day. Upon indicating which company one wants to visit, a paper printout carrying the visitor's name, a webcam photo and the name of the company one is visiting enables the visitor to pass the guards and enter through the gate. This process resembles procedures at 
national borders, wherein direct access is granted to the country's passport holders and temporarily limited access is granted to outsiders, upon registration with legitimising parties from within.

While the road is loud, busy and - during the monsoon season - muddy and dirty, the world within the compound appears quite different: guards guide pedestrians along neatly paved walkways and a park-like square with benches. A fish pond welcomes all who enter the compound and a small food plaza hosts an expensive coffee shop and a popular American fast-food chain. One must walk 400 metres across the office compound, along a palm tree shadowed corridor, to reach the Advice Company office building. When the sliding doors open, a cool, air conditioned breeze provides instant relief from the hot humidity outside. Before one notices the wide reception area with beige, shiny floor tiles and a big counter at which another security guard is stationed, one perceives the marked difference of the interior climate. Across the reception hall stands another guard, who asks for a badge before allowing persons to enter the elevator area.

To call the elevator, one must indicate the required floor on a small panel. With a "beep", a small screen indicates one of six elevators that takes the person to the desired floor without them pressing any further buttons. First-time visitors are immediately marked by their confused stance in front of the panel or their search for buttons to press in the elevator. When stepping out of the elevator, the temperature drops by another five degrees. On the right side, a sign indicates the washrooms; upon turning to the left, one steps into the reception area - a 40 square metre windowless room with the same shiny beige tiles as used on the ground floor. The area is dominated by a massive welcome desk that nearly spans the full length of the room, behind which a watchman in uniform and a female receptionist in an Indian kurta or salwar kameez sit. The rest of the room features television screens, a water dispenser and comfortable chairs. To enter the office area, one must cross the room along the reception desk, literally passing the two gatekeepers, in order to reach a 1.5-metre wide door of frosted glass. To unlock it, one must swipe his or her badge against a small panel and, following a single "beep" sound, pull the door open. The walls carry Advice Company's logo and the video screens show trailers about Advice Company's services and display quotes from clients. Visitors are asked whom they want to see and are told to take a seat.

On the first day of my fieldwork I was also asked to take a seat as it would take a while for the HR representative to pick me up from reception. Meanwhile, a young man offered me a coffee in a small china cup, and I used the 
opportunity to observe the reception more closely: Each time one of the elevators arrived, a group of people would pour from around the corner, either into the reception area or towards the washrooms. The washrooms served, as I later learned, as a transformation space following the commute into the office. The majority of the people who reached the reception area had a badge carrying their photo and name on a lanyard keychain with Advice Company's logo. They would greet their two reception colleagues, go straight to the frosted entry door, swipe their badge in front of the small control panel and walk into the office area. Even when the first person in a group had unlocked the door and held it open for his or her colleagues, subsequent persons would swipe their badge; the beep sound could be heard several times, legitimising each person's passage through the door.

Others had badges without a photo or a name; these badges had the word "Consultant" written on them. Persons with these badges would stop at the reception desk and write into a blue book lying on the counter. Following this, they would proceed to the frosted door, swipe their card and enter. On my first day, a group of three people came from the elevators with a pass that had been printed at the entry booth of the compound (similar to the one I had obtained in order to pass through the pedestrian gate from the street). These persons approached the woman at reception and stated the name of the person they wished to see, just as I did. The receptionist picked up the phone and called the colleague. The man in uniform opened a drawer behind him to fetch three badges with "Visitor" printed on them. He passed them over the counter to the three persons and asked them to sign in to the blue book. He also offered them coffee. A few minutes later, the frosted door opened and two persons with names on their badges entered the reception area. Handshakes were exchanged and introductions made, as not everybody seemed to know each other, and the group moved amidst laughs and lively conversation through the frosted door into the office.

Two females appeared, both with a photo badge in their hands. But instead of going straight to the door they stopped at the desk and wrote into the book without any intervention from the watchman. Only at this moment did I realise that there were two books - a blue and a black one. This time it was the black book that the two ladies wrote in. They were each handed a "Consultant" badge and then they proceeded on their own through the door. A young man with a big box came to the reception and talked to the watchman, who checked the box, picked up the phone and said a few sentences. Then he stood up and accompanied the man with the box through the glass door. Both 
returned shortly after and the man disappeared around the corner towards the elevators.

The reception phone rang and, after a short conversation, the woman at reception nodded at me with a smile. This motivated me to get up from my chair. I was asked to sign in to the blue book. The column headings indicated that I should enter my name, company, visit purpose, person visiting, phone number and entering date and time. As I filled in this information, I was handed a badge with "Consultant" on it. I used a few idle moments to look at the black book that the two ladies had signed in to and realised that it had fewer columns to fill out: only name, office location, date and person visiting. I was later told that the black book was for employees from different office locations. But before I was able to think more about the two books, the frosted door opened. I was greeted by the HR colleague whom I was assigned to for the first few days. We exchanged a few polite words and entered through the door. Here, the beige tiles were replaced by a dark grey carpet and we walked along a 15-metre long hallway into the open office area.

My observation of physical entry procedures at the office's reception provided insight into Advice Company's production of differentiation to the environment, as well as other systems that the company regarded as relevant. The ethnographic material allowed me to analyse the demarcation of organisational boundaries and the multi-layered structure of the organisational context. The next pages will provide a more detailed interpretation of office access. The various levels of organisational membership will be identified and introduced, and this discussion will be followed by reflections on the temporal and linguistic organisational boundaries.

\subsubsection{Producing differentiation along the three gates}

The massive security protected entry gates depict an explicit territorial differentiation between the office and the street. This system differentiation occurs with respect to the management of the compound and the maintenance of the walkways. While the compound itself is not a social system - just as the residing companies are not sub-systems of the compound - it nevertheless contributes to the differentiation strategies and entry procedures that facilitate Advice Company's system-environment differentiation. For this reason, I discuss the entry procedures here, in detail. The first distinction between individuals with or without a badge is executed at the entrance of the office compound. Here, it is irrelevant which of the residing companies issues the 
badge, as any relevant badge is equally qualified to grant its holder access, whether or not it displays a name and a photo. The word "Consultant" together with the logo of an appropriate company on the badge suffices, at this first gate, to enable the badge holder to pass from the street into the office compound. This shows that no differentiations are made with regards to the type of association with an organisation. Whether the badge is of the individualised or generic type, access to the office compound is given, both to members and to associates of residing organisations, who are able to produce this general symbol of organisational membership. My Advice Company badge indicated, on the backside, that the badge was the property of Advice Company and, if found, must be returned to the organisation.

In contrast to the badge holders, members of other systems in the organisational environment are allowed temporary access to the premises with a visitor pass, which is printed on paper. This pass must be returned to the guards when the visitor leaves. Irrespective of the nature of interaction with Advice Company, which might be a client meeting, a delivery of ordered goods or a job interview, any non-badge holding individual is classified as a visitor. Persons who are unable to qualify their interaction with one of the residing organisations are not allowed to enter the compound.

Especially during the first weeks of my research I perceived this boundary as extremely salient. Entering the calm and well-groomed compound from what seemed to me an irritatingly loud, chaotic and crowded street felt a bit like arriving at a safe harbour after an adventurous journey. Only gradually, that notion changed to the point that I felt comfortable enough after a month to regularly stop in the morning at the small stall selling green coconuts to enjoy a first treat of the day, while observing the entertaining street scenery. It took another month for me to learn to appreciate the various street food snacks offered in great variety in front of the gates to the compound, which the colleagues I accompanied introduced me to as an alternative lunch or predinner option. As I rushed in and out of the compound in the beginning, the perception of the gate area as a clear-cut dichotomy between chaos and order gradually decreased in salience, and the street area in front of the gates became a place for me to hang out before and after office hours, or even during the day on chai breaks.

At the second checkpoint, right at the entrance to the office building, the display of a badge from one of the building's organisations or a visitor pass with an appropriate company name must be produced for a person to gain access to the elevator calling panel. I only became aware of the fact that entry 
into the office building constituted a further differentiation several months into the fieldwork. In my daily, semi-automated coming-to-office routine, I once mistakenly entered the neighbouring office building, which looked identical but was 50 metres up the palm tree corridor. I did not even make it to the second step towards the sliding doors when the watchman stepped out of the hall and pointed me determinedly towards the next building's entry. The colour of my lanyard keychain was different to that of the employees of the company residing in that building. Therefore, he had evidently been able to identify me as a non-member from far away.

Figure 3: Map of entry procedures to the main office

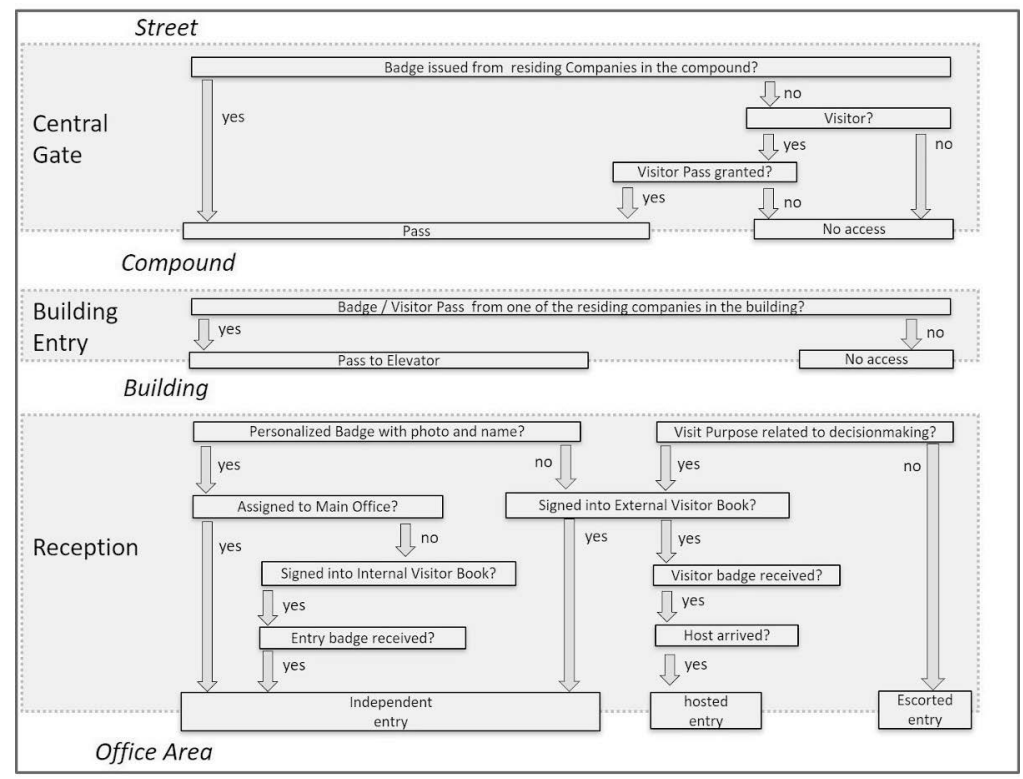

In contrast to the first two gates, the office reception of Advice Company performs significantly more sophisticated procedures of distinction, representing a number of the organisation's decisions to produce system-environment differentiation through the use of multi-dimensional access procedures. Figure 3 provides a logical illustration of the access sequence with the relevant decision parameters. The grey blocks represent the three gates: the central gate must be passed from the street into the compound; the second gate 
occurs upon reaching the office building; and the third gate is found at the organisation's reception. The logical process illustrates the decision tree upon which differentiation is produced at each gate and shows the granularity of decision processes at the reception. Based on these decision processes, I will describe in the next section the various groups that are marked by distinct decision processes at the reception and can therefore be regarded as relevant for further analysis.

\subsubsection{Differentiation at the reception: Members and other groups}

This section illustrates Advice Company's differentiation strategies relating to entrance into the office, as observed at the reception. The entry procedures at the main office contribute to the organisation's system-environment differentiation and reveal the relevant types of organisational membership. Similarly, members of interacting systems in Advice Company's environment are provided access on the basis of their relevance to the organisation.

Entry for organisational members

Individuals carrying a badge with a photo and a name are permanent employees of Advice Company. This means that they have been selected as members of the organisation and are bound to Advice Company as official employees, by contract. They are "on payroll" and eligible for social benefits such as health insurance for themselves and their family. The process of gaining organisational membership is discussed in more detail in Section 4.3. If an employee's primary work location is the main office, he or she does not need to sign in upon arriving and can enter the office directly by swiping his or her badge in front of the control panel at the frosted glass door. The control panel has the primary purpose of unlocking the door, and a similar panel is installed on the other side of the door to unlock it for those who wish to leave the office area. The panel is connected to a software that registers the entry and exit events of each badge. Employees are instructed to swipe their badge each time they pass through the door, for security reasons: in the event of a fire or any other emergency, this practice enables rescue teams to have an indication of who might still be trapped inside the office area. Therefore, when passing through the door, each individual swipes his or her badge, producing the beep sound. This is also done when employees go to the washrooms next to the elevators, instead of using the ones located within the office area, or when they sneak out for a quick cigarette break. This act of swiping the badge marks the or- 
ganisation's territorial borders and is performed an estimated thousand times per day. While this check-in/check-out procedure is both a visible production of system-environment distinction and furthermore accentuated by the audible signal of the "beep", it also illustrates the organisational understanding of the distinction between workspace and non-workspace.

Compared to other means of entering, this mode of access into the office area clearly stresses the status of full membership to the organisation. This status, which allows persons the simplest, most direct entry, is only granted once an individual passes the elaborate decision process that grants him or her membership. This full membership status is furthermore highlighted by the fact that it is a sine qua non conditio to serve as a host and bring visitors into the office.

Employees based at other office locations of Advice Company must sign in to the employee visitor book. These employees receive a badge to open the door and then are able to move freely in the office area. This act of internal differentiation reinforces the already established boundaries with the environment. It enables a setting of reduced complexity (Luhmann 1995a: 189), as symbolised by the employee visitor book, which requires significantly less information than the book that non-members are required to fill in. At the same time, it reinforces the boundary with the environment by executing and therefore highlighting an internal sign-in procedure, in contrast to external sign-in procedures (ibid.: 193).

"Visitors" with a badge: The temps

Those with a "Consultant" badge are required to perform another mode of entry. These individuals must, on the one hand, sign in to the blue book each day like a visitor; but on the other hand, they are able to pass the frosted glass door on their own and enter the office space independently, similar to employees. Such colleagues are predominantly on temporary third party contracts and therefore not official employees. The hybrid nature of their roles, resembling a liminal status of not belonging to the organisation yet involving tasks that are similar to those who are full members, has been demonstrated in other organisations (e.g. Garsten 1999) and can be illustrated by this mode of gaining access to the main office. The badge allows these persons entry into the compound and the office building, similar to permanent employees. At the Advice Company reception, however, their status as an organisation-external element of the workforce is reproduced by the sign-in process every morning. 
For the duration of the research, I received such a "Consultant" badge; hence, the sign-in procedure was part of my daily routine. I would often meet a colleague on the walk to the office and start a conversation with him or her, but this conversation would be abruptly stalled as soon as we reached the reception. While I would stop to pick up the pen and sign in to the blue book, the colleague would walk on towards the glass door and our conversation would come to a sudden end.

\section{Priority visitors: Clients}

In the description of the reception scene I recounted my observation of a group of three people who were handed "Visitor" badges and asked to sign in to the blue book for external visitors. These individuals were members of a different organisation and thus Advice Company clients. Such external visitors are only allowed to enter the office when accompanied by a member of Advice Company who has been assigned to manage them within the environment. The visitors must indicate these persons as their hosts in the visitor's book.

In spite of their position outside of the organisation, visitors are an important category with regards to their influence on the work conducted within it. They are kept waiting for minimum periods and are even quoted in the video clips played in the reception area.

Low-interaction visitors: Service and support staff

In the case of the young man who delivered a box of leaflets, the purpose of his visit was not connected to decision-making - the central constituting operation of the organisation. Therefore, he was not asked to sign in to the visitor book or hand over a badge. Instead, he was accompanied by the watch guard. Once he delivered his package, he disappeared again. This is an example of an interaction of the organisation with its environment, which supplies a necessary service to the organisation without having a direct impact on its structure.

\subsubsection{Territoriality: A merely symbolic value?}

Luhmann classifies territoriality as a type of "meaning-constituted boundary" with only minor importance for modern organisations, asserting that territories have a mere symbolic meaning (Luhmann 1995a: 194). As I illustrated above, the frosted glass door symbolises the entrance into the centre of the 
organisation: guarded by two gatekeepers and elaborate entry procedures, it constitutes a strong demarcation of the organisation from its environment. Furthermore, each act of entering into that space becomes part of the discourse on the organisational boundary. The differentiation strategies that are expressed through the entry procedures at the reception recur inside the office space through the display of distinct badges for temporary contractors and the constant supervision of clients by their hosts (or the parcel delivery man, by the watchman).

Chapter 5 addresses the corporation's three offices in the city and their relationship in the context of the broader organisational structure. With respect to the negotiation of organisational boundaries through territoriality, the set-up of Advice Company's street office - the smallest of the three locations - builds an ostensive case. Located in a two-storey, windowless brick house of approximately 150 square metres on each level, the office is characterised by a strict division between the ground floor and the upper level. The air conditioned ground floor features two office spaces with desk bays, computers and other office equipment, such as printers, phones and office chairs. The upstairs area is of comparable size, yet is equipped fundamentally differently: the rooms are vested with 40-centimetre wide plain tables, which are set up along the walls, and white plastic chairs. Three phones and a printer serve as equipment, and there is a tiny room for meetings. The area does not provide individualised workspaces and the air is hot and humid, as there is no air conditioning. The ground floor hosts the office for Advice Company's employees, whereas the upstairs rooms are the work environments for the "freelancers". Freelancers are recruited on a project basis to collect data required for a client project. I discuss their role in Section 4.2.2; at this point, I would like to use the street office as an example of a clear demarcation of territory, in connection with organisational boundaries.

The "freelancers" are not formal members of the organisation. However, this fact is not emphasised through the use of different badges (as freelancers do not receive a badge) or sign-in procedures, as practised at the main office for temporary employees and visitors. Instead, the distinction is marked by the allocated space, which is unmistakably distinct from the space occupied by internal employees. The organisational boundary is furthermore established by the convention that freelancers do not enter the ground floor office area. Rather, when Advice Company employees want to talk with one of the freelancers, they go upstairs rather than asking the freelancer to come down. 
This aspect of the office as a territory that distinguishes the organisation from its environment can also be illustrated by the daily physical and temporal efforts required by the individuals to overcome the border between their home and the organisational space. Commuting is a central topic of casual conversation in the office. Whenever I engaged in a conversation with a new interlocutor, often the first questions asked would be: "Where are you put up?" and "How long did it take you to travel into the office?" "By which means of transportation?" Notably, geographical distance to the office in kilometres was not of high informative value in my answers, as the evaluation of commuting was based on the availability of public transport connections or the proximity of one's home to major motorways that one could take to reach the office.

When I arrived for the pilot study I was convinced that I had made a smart choice to stay in a hostel "only" 10 kilometres away from the main office location. The first morning, I learnt that the road leading to the office was a central connection between the eastern and western parts of the city. As no train service covered that area, the road seemed to be perpetually congested. It took me more than an hour to travel that 10 kilometre stretch, and I quickly learned that this put me on the lower end of the commuting time range experienced by my colleagues. Right at the beginning I was told that commuting times usually varied from 60 to 90 minutes, but could easily reach two hours at peak traffic times or during heavy monsoon days. Not all employees, however, commuted for several hours each day. Those who could afford it or were flexible ${ }^{1}$ would move to one of the neighbourhoods in close vicinity to the office. As a consequence of my commuting experience during the pilot study, this was my strategy, too.

Lamentations about the commute were very common, as the employees travelling by overcrowded buses or trains described it as a physically exertive act, and those coming by motorbike or car classified it as mentally stressful. Ruchika told me that she had a car at home but preferred to take a rickshaw each day for 90 minutes, as she did not feel comfortable driving in what she referred to as "chaotic and sometimes aggressive traffic during rush hours", despite having tried several times. Anyone who has experienced the traffic in India's mega-cities during peak times will probably relate to her sentiments.

Not only driving, but also commuting by train was described to me as unpleasant. While the benefit of trains was the mostly predictable duration and therefore had no obligation to live in their family households. 
of travel, Ananya from the city office stressed the physical effort it required to stand tightly pressed against others in a sweaty crowd: "It's an involuntary full body massage!" Others mentioned the extreme caution and aloofness needed to avoid fights on trains and the tracks, especially in the evenings, when all of the commuters would be tired after a long day. When leaving the office, the ladies were not the only ones to gear up for their commute with more robust shoes and modest dress: several men would also switch their shoes and glasses for travelling. Even when taking a comparably comfortable rickshaw, ladies would wear a scarf tightly fixed around their head with only a small gap revealing their eyes. The reason for this habit, I was told, was to prevent inhalation of the polluted dust on the street, which stuck tightly to sweaty skin; it also prevented the face from tanning due to exposure to sunlight.

The notion of the commute as a border between work and home is also symbolised in the transformation processes that occur in the washrooms right next to the elevators before one proceeds towards the reception. Women take small pouches of vanity sets from their handbags or backpacks to wash off the street's dust, redo their eye make-up and hairstyle and apply deodorant. Some change their shoes and others put on a dupatta (scarf that matches their outfit) to complete their dress. During monsoon season, sometimes even an entire outfit must be changed because it has got completely drenched. Such an experience once led me to carry a spare set of clothes with me to the office.

While the commute to the office is an evergreen topic of conversation, it becomes a serious concern for employees during the peak of the monsoon season. When streets are flooded from several days of constant, heavy rain, the traffic situation aggravates to even longer traffic jams and more unpredictable road conditions, and this leads more people to turn to public transport. The high demand results in rickshaw drivers becoming more selective of the customers they accept for a journey. Waiting an hour for a rickshaw driver willing to provide a ride to a relatively close (i.e. unprofitable) destination is a common situation during these weeks. Those who depend on train services are faced with the prospect that trains might encounter technical issues that will leave them stalled for several hours. The organisation recognises the commute as a serious obstacle, and this is reflected in office-wide emails that are sent on days with exceptionally heavy rain forecasted. These communications prompt people to leave the office early, "preferably by $4.00 \mathrm{pm}$ ", and allow all employees to work from home the next day.

Both the commuting as well as the split office structure suggests that territoriality has not only a symbolic value in relation to organisational bound- 
aries, but also a structural value. The Advice Company employees perceive the commute as an activity requiring substantial resources. Reversely, the organisation marks - through its email communications - its awareness of the potentially hazardous travel home from the office during the monsoon season, and even suspends its territorial focus for the next day by offering all employees permission to work from home. Similarly, the separation of the city office into an internal space and an exchange area for freelancers goes beyond symbolic meaning, enabling a structural frame for cross-system collaboration (see Chapter 5, Section 5.4). Therefore, I argue that - in this case - territoriality fulfils significantly more than a symbolic function for the demarcation of organisational boundaries with the environment.

\subsubsection{Temporal boundaries}

Organisations not only differentiate themselves from the environment in terms of membership and territorial boundaries, but also through time dimensions. Luhmann speaks of the emergence of a system-immanent time that must also fit the time of the environment (Luhmann 1995a: 185). While this idea is particularly important for analysing how the organisation translates input from the environment (i.e. from clients) into its own context and how time is used to produce internal differentiation (see Chapter 9), time dimensions can also be analysed in the context of their effect on organisational boundaries.

When I heard that some colleagues occasionally came to the office on the weekend, I realised that I had taken my own perceptions of temporal boundaries in a work environment for granted. At the next opportunity, I sought approval to come to the office on the weekends, as well. The office life on Saturdays resembled that of early morning or late evening weekdays, with only the watchman as a gatekeeper at the reception, the canteen closed and around 50 to 80 people working at the desks. Sundays, however, felt significantly different: the frosted glass door would stand fixed wide open and the air conditioning would be switched off. The watchman would not wear his usual uniform, but a stylish casual outfit with a flat cap. I would not have to sign in to the visitor's book, which was on all other days obligatory. Several cleaning personnel would be busy vacuuming the carpet and wiping off the desks, which would produce a comparably loud and unsettled environment. I visited the office on about 10 Sundays and never saw more than a dozen colleagues. Those who came to the office on a Sunday would also be dressed 
very casually, some even in board shorts and flip flops - an absolutely inappropriate and impossible attire during the week.

The activities of the cleaning team and the dress of the watchman emphasised that this was "not really a work environment", as one of my HR contacts remarked when I requested permission to come to the office on this day. The location was not able to serve the organisational members for their work tasks on Sundays. Rather, the office would be in a state of resetting and preparation for the new week to come, with several cleaning and maintenance activities being performed. Sunday marked the temporal boundary that the organisation had set up to distinguish workdays from work-free days. Despite the fact that several employees apparently worked in the office on Sundays, their extremely casual dress accounted for that notion.

\subsubsection{Linguistic boundaries}

When walking through the main office, one notices the broad range of languages spoken. As the colleagues of Advice Company originate from all across India, local languages such as Bengali and Tamil can be heard, as well as English and Hindi, which serve as the two linguae francae in this context. But while Hindi is associated with the verbal internal office conversation, all written communication and any communication with clients is strictly conducted in English. This emphasises not only the more formal mode of communication but also the perception of the organisational boundary.

In contrast to the office communication, conversations at lunch time were occasionally difficult for me to follow. While at the beginning of the lunch hour conversations would mostly be held in Hindi, the conversations would often drift - depending on the composition of the group - towards Marathi or other languages. In the beginning I perceived this as irritating, but I came to realise that this practice showed that the use of more local languages marked a situation as "private", in contrast to the use of English or Hindi, which marked official work, as pre-determined by the organisation. The switch between languages furthermore demarcated the boundary (as perceived by the employees) between work settings (which were thought to be of interest to me and my research project) and non-work situations (which were assumed to be uninteresting to $\mathrm{me}$ ). 


\subsection{Differentiated environment: Clients, freelancers, universities, contractors}

Drawing on my observations of the access procedures at the reception of Advice Company's main office and the organisation's manifestations of territoriality, I illustrated in the previous section how the organisation produces system-environment differentiation and (re-)enforces its boundaries. This section focuses on the organisation's environment - in particular, systems that are of direct relevance to Advice Company: its clients, its freelancers and universities. Contractors can be regarded as a fourth system, yet they occupy an opaque state. They are positioned in not only a perceived liminal state towards full organisational membership (as suggested in Section 4.1.2), but also a "betwixt-and-between" position, between the organisation and its environment (Garsten 1999: 604). Continuing to apply the theoretical framework of Luhmann, I will structure the analysis by questioning which elements in Advice Company interact with the environment. I will then analyse the transactional openness of the system, how the organisational boundaries are constantly negotiated through communication, how the input from the environment is interpreted or translated into the organisation and how the environmental systems influence organisational functioning, and/or vice versa.

\subsubsection{Clients}

Clients are members of other business organisations who purchase the services of Advice Company. They constitute the most influential system in the organisation's environment. Clients' decisions to spend monetary resources on Advice Company products - mostly in the form of projects - constitute the key input for the system, triggering a range of decision chains. Consequently, "the client" is the central focus of decision processes in the organisation. While basically all elements of Advice Company are directly or indirectly involved in client projects, and "client centricity" is an often-used buzz word in the office, only a few functions in the organisation interact with clients directly. Such direct interaction with clients is performed exclusively by specific roles within Advice Company, namely top managers and client consultants. These roles function as a specialised membrane to manage interaction with these environmental systems, and are the most visible roles in connection with the organisational "boundary work" (Holtgrewe 2003: 64). But belonging to the client consulting team is not the only requirement for managing client rela- 
tionships. The right to interact with clients is subject to organisational control, and the level of interaction - from emails up to active participation in presentation meetings - is related to both one's position in the hierarchy and one's tenure in the organisation. This produces a distinctive internal differentiation of individual client consulting teams and provides a good example of how the organisation's decisions in relation to the demarcation of boundaries with the environment relate to internal structures.

The status associated with client interaction is suggested by the words of Kashish. He had joined the client consulting team only a few weeks before I accompanied him for the first time. When I asked him to describe his work to me, he answered: "The work I am doing now is only the operational part. But my actual job is the client side, so once I have learned enough I will be talking to the client, maybe in a month or so." Kashish's response implies a difference in status between "only operational work" and client interaction, with the former deemed an activity of lower value. The statement of his manager Bright on the same question mirrors this understanding from the opposite perspective of the team's internal hierarchy: "whenever there's a client interaction I am involved, but the operational part [...], I don't deal too much with this. My job is to deal with the client, so any major communication with the client."

Kashish understood that before he could interact with clients he would have to gain experience with the internal processes. In this way, he connected seniority to the right to engage in interactions with client systems in the environment. Both Kashish and Bright's statements position "operational work" at the extreme lower end of the consulting team's value system. These operational tasks refer to Advice Company's internal communication with colleagues in project coordination teams or, in crisis situations, even project execution teams. They denominate the opposition of client-centric operations and are associated with the value ground reality in the context of project execution. In the course of this book, I will show that these opposing values play an important role in the organisational system. In Kashish and Bright's client consulting team, information was clearly selected by orientating on client centricity. Although Kashish was a novice to the team, he had already applied the same selection mechanism as Bright, which suggests that the guiding difference of a social system shapes communication and not individuals.

Kashish and Bright's focus on client interaction stresses not only the desirability of the task, but also the internal observation of a strictly organisation-controlled boundary with the client. This is also reflected in Bright's notion that he, in his role as a manager, had to review and control the emails 
sent to the client: "We want to ensure that everything that goes to the client comes through me for review." Such self-determined organisational mechanisms controlled interaction with the environment and highlighted the selfobservation of the organisational boundary with the environment. Furthermore, the consulting team colleagues were very aware that that the selected information would be subject to a selection of understanding by a different system. Aniket, an experienced consultant, had very clear views on how the information should be shaped (i.e. selected) from the internal version to that of the client's system:

When communicating to the client, don't ever tell him you have issues on our side. There has always to be a different explanation to it. I would tell of issues with conversion rates being too low, or inflate small issues with the client's base data to be the key reason of delay. For example, we always have issue with the work teams in Chennai, so when we can, we try to get around being too dependent on them, but obviously without saying to the client that this is due to the internal issues in that city team. Of course we have to find other useful explanations that make sense to them. So when there are two comparable options we try to make them not selecting the issue-areas... because they wouldn't understand and get it all wrong.

Aniket's last remark, in particular, shows that his strategies of using specific utterances were concerned with information selection in a social system and not predominantly with achieving a competitively favourable position in front of the client. Similarly, the input in the form of project briefings received by the client representatives was subject to translation work performed by the translation specialists on the client consulting team. Their role required them to "understand the clients' needs", as Bright explained; they needed to be able to select an understanding of the client that would be compatible with the system's language, in order to deliver satisfactory projects. The ethnographic case studies presented in Chapter 10 address such translation chains and working misunderstandings in the context of client projects.

Understanding the "client's needs" through a client-centric orientation is a skill that is explicitly advocated by organisational leaders, for example during "town hall meetings". All employees in the main office are invited (or requested) to attend these events. At a "town hall" meeting, representatives of India's senior management team or executive board members from abroad stand on a small stage in the main office and present on topics that are relevant to the organisation. Their voices are transmitted via an audio system to 
the entire office area. Usually, "client" is by far the most frequently used word in these presentations. Only once, at the end of my research, did I attempt to quantify this observation: During a speech given by a senior manager from the US I counted how often he used the word "client". The talk centred on the topic of how he had previously, during his early years as a client consultant, played a personal game to "never say no to a client" and how this had made him successful. During his 12-minute talk, the word "client" was broadcasted 34 times through the speakers over the heads of the 450 employees across the office floor.

This central value client centricity is also implicitly present as a key trait of employees' "most remarkable projects". This topic featured in one of the standard questions I asked all of my interlocutors in the interviews. In particular, younger colleagues with limited work experience would choose to talk about a successful project and a few others would opt to describe a particularly disastrous client project that they vividly remembered. About two-thirds of my interlocutors, however, described a successful project within Advice Company that they had been involved in. These projects were remarkably similar in their plot of the interlocutor's intense effort in either a project in crisis status doomed to fail or a particularly challenging project requiring tricky solutions, which was ultimately fulfilled to the client's satisfaction.

Most remarkable project... hm... that was one I got directly after I joined the new team. Some escalations had happened and the client was really upset with us and our service. And then I was asked to take over. My achievement was to get the account [i.e. the client] really back on board again, up to the point that when they hear that I am on a project they relax, because they know all will be fine and taken care of. (Sakshi, Client Consultant)

I got a lot of client appreciation, although I don't have client interaction. But in [the] last one and a half years I got three projects where I actually received client appreciation. Often it's clients saying that "Your project coordination team has done a good job". They [the clients] do not know me, but still it's indirect appreciation. (Hitesh, Project Coordinator)

...and when I presented the project report to the client, he praised it, saying that we have changed the way presentations are being done, so I take a lot of pride in that. (Bright, Client Consultant) 
Getting positive feedback from a client for the good work one has done is proof of one's ability to "understand the client's needs" and fulfil the role of translator according to the organisation's expectations. Stories of particularly challenging projects that were mastered under the most averse conditions and resulted in happy clients are told and re-told among colleagues at team outings or prolonged lunch breaks, akin to tales of heroic deeds. Successful mastery of a challenging project associated with positive client feedback finds its most solid manifestation in the form of client awards. Awards can be objects such as trophy cups or laminated paper certificates carrying the client's logo and a description of the award (e.g. "2nd place most innovative project"). These awards are usually placed on desks, in open display. Internally, Advice Company also awards client-centric behaviour. Such awards are handed over as laminated certificates, together with a bank voucher of a few thousand rupees (approximately 15-60 euros), depending on the level of the award (bronze, silver or gold). This award system is an example of the structural coupling of the organisation with the client systems in its environment.

Furthermore, being close to a client and getting appreciation from this external system influences more than simply one's status inside the organisation. "Moving to the client side" is an often-used phrase referring to the career aspiration of gaining a position in a client's organisation. This is a career target voiced predominantly by employees who interact with clients - those who are already as close as possible to a client as a member of Advice Company can be.

Employees' desire to gain a role with client interaction reveals the organisation's client-centric orientation: those who lack contact with clients strive for jobs in functions associated with client interaction. The client system therefore strongly influences the career aspirations of Advice Company employees, and, through client appreciation (i.e. via an external system), internal status is gained. As I will show in Chapter 9, this client-centric orientation is connected with the corresponding value that aligns the organisational structure with cross-departmental work processes on client projects, along the client centricity paradigm. As a consequence of the client-centric orientation, the client system receives such a high status that it constitutes a threat to the organisational membership decisions of Advice Company's employees. The desire to "move to the client side" (i.e. to work in the client's organisation as an employee) is an example of how the environment influences the organisation to the extent that membership can change. Two and a half years after I completed my fieldwork in June 2014, several Advice Company employees had 
moved to roles within client organisations. During a skype call with Kashish two years after I completed my fieldwork, he told me he was happy to have "managed the switch to the client side". Sakshi used to be a consultant at Advice Company and is now an employee of a former client of hers, and Raveena, from the same consulting team, also works at a client's organisation.

\subsubsection{Freelancers}

Although I did not see freelancers during my observation at the reception of the main office, I heard about them on the first day of my fieldwork. Freelancers are external to the organisation, yet they significantly contribute to Advice Company's project development process through the completion of work tasks such as data collection, which subsequent processes build on up to the final project delivery. Freelancers are paid on the basis of the work packages and data they deliver and their position outside of the organisation is clearly marked by their different, less equipped office rooms in the street office, in conjunction with the fact that they are not provided with an email address or an Advice Company badge. The vast majority of the freelancers have other jobs beyond the work they do for Advice Company. Due to the unpredictability of incoming projects, they are taken on and off jobs. Therefore, Advice Company's employees assume that some freelancers work in parallel on projects with competing organisations.

Similar to my interaction with clients, my direct interaction with freelancers was limited. I accompanied employees who had the specialist function of managing interactions between Advice Company and the freelancers. These colleagues held specific roles within the project execution department concerned with organising freelancer teams. I participated in their meetings, observed casual updates between the employees and the freelancers and reviewed interlocutors' email communication history with freelancers on a project. Employees in this function had often started as freelancers, themselves, before becoming members of Advice Company. Therefore, one of my interlocutors organised an opportunity for me to accompany two freelancers for a day to experience "that part of the ground reality", as one of my interlocutors expressed it.

In contrast to client centricity remains ground reality as a term less explicitly tangible. This is partially rooted in its subordinate position in the organisation's discourse but also in the reason that I decided to subsume the different unlabelled notions of counter-currents to client centricity under the term 
ground reality. Therefore differs the meaning of ground reality in relation to the context. The most explicit use of the term I encountered in the context of work with and from the freelancers. Here ground reality referred on the one hand to the data the freelancers deliver and the conditions under which they are produced. On the other hand ground reality was used to describe a distinct and less predictable mode of operation. In this case it encompassed the freelancers-teams and their team managers within Advice Company as a source of disturbance for the client centric processes. An example for this notion became salient during a lunch chat with project coordinator Sheeba and a colleague from the finance department who had joined us spontaneously. As I had not met her before I started to ask the colleague about her job and her current work. I did not get far, as Sheeba cut across my questions with a playfully exaggerated envy in her voice: "She is lucky, she doesn't have to deal with the ground reality, so her world is fine!"

Having to deal with the ground reality therefore required distinct skills. While the client consultants are experts in translating clients' strategic needs into projects, a similar translation is necessary for information to be processed by freelancers and vice versa. For compatibility with the "ground reality", information about projects must be reduced to the minimum and limited to the absolute basics required to perform the respective work task. The need to select information and a message for freelancers is commonly acknowledged within the organisation, often with the connotation of simplification or reduction, in Bruno Latour's terms (see Chapter 10, Section 10.4 for a discussion of projects, translation chains and the "ground reality"). I will show, however, that the translation processes from freelancers in the environment to Advice Company employees are at least equally relevant, although this fact is overseen by the organisation's dominant discourse of client centricity.

The structural set-up of the street office also depicts the functional need to manage communication processes with freelancers. As illustrated above, the organisation manages its interactions with client systems in the environment via the specialised function of client consultants. Much as the main office provides a space for client interaction, the upper level of the street office serves as a zone in which Advice Company controls the exchange between the organisation and freelancers. The employees who manage the interaction with freelancers use a small room on the upper level for briefing meetings and spontaneously go upstairs to informally check on their freelance team's current situation and any potential issues they may have encountered. Detached from individual communication, the upper level facilitates exchange in the 
most literal sense: the freelancers are equipped with laptops for their work and send the data they collected during the day through the organisation's network and get the most current updates on their work packages in return.

Such exchange zones facilitate the interaction between different systems and are often part of contemporary corporate offices. These zones are office "conference centres" and provide a number of meeting rooms with less restricted access than the rest of the office building, where employees' desks are located. In these zones, meetings are held with members of environmental systems, such as clients, suppliers and partners. These areas also commonly provide an Internet connection that can be used by guests, giving them access to email but not to the organisation's internal network. Advice Company's main office also features a large, well equipped meeting room in the corridor that leads to the desk area.

The function of such conference centres is indeed congruent with the function of Advice Company's upper level freelancer zone, which has the purpose of facilitating system-environment interaction and manifesting boundaries. However, the corporate conference centres and the freelancers' zone are, however, located on opposite ends of the prestige continuum. Conference centres boast state-of-the-art technical equipment, stylish design furniture, generous spaciousness and often a tray of coffee and biscuits. The freelancers' space in the upper level of the street office, in contrast, is vested considerably more simply than Advice Company's workspaces in the main office: it was notably geared up for the practical aspect of system-environment interaction rather than image concerns. Correspondingly, the set-up of the street office is seen by the freelancer communication specialists within Advice Company as the most suitable location for inter-system interaction:

You know, here in that more simple environment it is just very easy for the freelancers to come in and catch up. I can't imagine them do[ing] this when they have to stand in line to at the entry gate [to get a visitor's pass] and wear a badge every time they come to the main office. Here is such a big in and out of people in the course of the day, that always doing registration and these things would create a big issue. (Payul, Freelance Work Coordinator)

The main office would not work, it is too far out for the freelancers, difficult to reach. They would have to spend at least 20 rupees per day on travelling, that is too much. (Rohan, Freelance Team Manager) 
These quotes refer first to the benefit of the office being located in an area they consider a "suburban setting" next to a train station. The second aspect of the street office that employees believe makes it a particularly suitable setting for cross-system interaction is the physical space that is not marked as an official area of Advice Company, and thus does not require complex access procedures. The upstairs freelancer zone stands in contrast to the downstairs area of the Advice Company work environment. This territoriality, as well as the perception of freelancers as outsiders to the organisation, was explicitly marked in a situation when a young man (who I later learned belonged to a freelancer team) was folding a few cables into a box in the downstairs area. Suddenly, one of the team managers rose from his desk, walked over to the young man and asked him what he was doing. The young man pointed to the box and said he had packed the cables for a project, just as he had done the week before. At that moment, the manager raised his voice and scolded the young man for touching Advice Company's property without asking permission. Just because the young man had been given permission the previous week did not suggest that he would be granted the same now. The uncontrolled activity of the young man and the exhibited agency without prior permission within the territorial office area of Advice Company constituted - in the manager's perspective - a border infringement. Through the manager's communication, he re-established the organisational boundary with the freelancer's system (as represented by the young man), both in territory as well as in freedom of action.

The freelancers comprise a system in Advice Company's environment that is clearly demarcated through the fact that neither badges nor official email addresses are provided. Furthermore, a specific functional role within Advice Company is assigned to the boundary work with this system, and employees within this function serve to manage the relationship in the organisation's favour. Despite holding positions outside of the organisational boundaries, the freelancers are involved in the project development process and Advice Company is dependent on their labour services and the data they provide in order to maintain itself. The split-level set-up of the street office reproduces these system interdependencies and recognises the need for structures that foster interaction with the environment. It is interesting, in this respect, that both the intersystem dependencies and the organisational structures and functions that manage the relationship with the freelancer system are similar to those employed for the client system. Nonetheless, the two systems are located on opposite extremes of the status scale (Figure 4). 
Figure 4: Structural similarities: Clients vs. freelancers

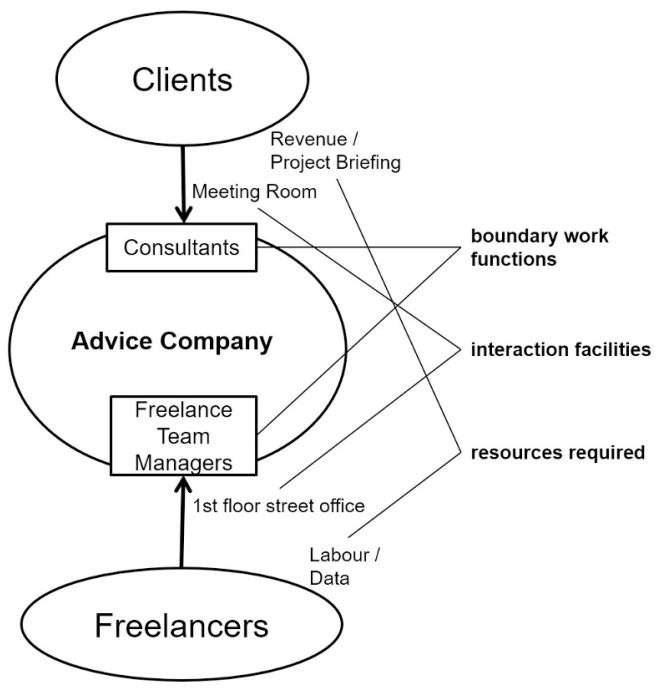

\subsubsection{Universities}

While interaction with clients and freelancers is central to the company's core activity (the planning and execution of client projects), interaction with the higher education system serves to ensure sufficient membership candidates with the traits desired by the organisation. Similar to Advice Company's interaction with clients and freelancers, the organisation's interaction with the educational system is also performed by specific members of the organisation - in this case, those in the HR department. The basis of this interaction is a recruiting partnership between Advice Company and particular universities, which allows Advice Company to conduct membership selection events on campus. The universities, in return, market these events as attractive opportunities for prospective students.

The higher education system in India comprises a large network of over 600 universities and 33,000 colleges, both public and private. The latter have risen from virtually none in the 1980 s to 90 in 2011 , in response to increasing demand for higher education (Tilak 2013: 6). Along the same line, degrees and 
courses have increasingly accommodated the demands of prospective (and paying) students, resulting in more IT-related undergraduate courses, such as electrical engineering and computer science, and more postgraduate courses in management studies. In parallel, enrolment in humanities and social sciences programmes has decreased (ibid.: 8). There is little literature available on the way in which business organisations have influenced and shaped India's higher education system. An employee of Advice Company with whom I talked about the higher education sector voiced the opinion that due to broad differences in educational quality across the country, "campus recruitment" is necessary for channelling opportunities for entry into the organisation.

The campus recruitment events function as follows: When students are approaching graduation, organisations with an established partnership with the university organise recruitment events on campus and use these events to hire new employees. Prior to coming on campus, the organisations announce the types of job on offer, including salary ranges and number of placements available. At the Advice Company event, the HR colleagues (who serve as representatives of the organisation) give a brief introduction to the organisation and the job on offer to the students. Then the interested students take the company's standardised written entry test; those who pass are shortlisted and subjected to two rounds of interviews. At the end of the day, either the new recruits are directly offered a job or a few candidates are invited to one of Advice Company's offices for further job interviews. Hence, a major part of the recruiting process occurs on university premises and is performed by members of Advice Company with the aim of selecting members of the university who have chosen Advice Company as a potential employer from a number of other companies also present at the campus event. On these recruitment days, the focus is changing organisational membership. However, while it seems that this day is primarily one on which decisions are taken, it merely represents the culmination of a chain of decisions from different directions, coming together.

First, the decision of Advice Company to establish a particular campus recruitment partnership is based on the university's ranking. Second, Advice Company decides which job(s) - and therefore which entry-level salary - will be offered to graduates of that university, and this is again dependent on both the university ranking and the current needs of the organisation. A few weeks prior to the campus event, Advice Company decides how many job placements for each role they will offer and announce this number on campus. Only a few of the top 10 universities are involved in the executive trainee programme 
for future leaders of Advice Company, while graduates of universities at the next level have the opportunity to secure consultant roles and students from lower-ranking institutions (ranking between 50 to 110) have the chance of becoming support analysts or project coordinators. Graduates from even lowerranking universities can become coordinator assistants or be hired on temporary contracts. In this way, the environment strongly shapes the way in which employees join the organisation. This external hierarchy also finds its reflection inside the organisation, where employees proudly mention the names of their alma maters. If I did not react with the appropriate sound of admiration when my interlocutors spoke of their graduating institutions, they would often point out that theirs had been a premium institution. On several occasions, I was informed about the top institutions for higher education in India and how difficult it was to gain admission to them.

Some of the employees described to me how - even years before they had filed an application for Advice Company - their aim of becoming a member of the organisation drove their decisions. The business schools in India charge significant tuition fees and administer various admission tests. Based on the number of university applications a person files and his or her test outcomes, he or she might be offered placement at a number of business schools. Universities are expected to state the expected starting income package and the placement rate of their graduates, together with the companies their graduates are employed with. From this information, potential students make a return-on-investment calculation before they enrol. In many cases, the hiring partnership with Advice Company constitutes a major decision parameter for the students who want to work in Advice Company's industry, as access to the organisation is deemed more achievable through campus recruitment events (see also Garsten and Nyqvist 2013:10). Since university ranking influences the particular jobs and salaries offered by advice Company, potential students also take this into account - as do universities, who adjust their tuition fees accordingly. This illustrates the interdependency between Advice Company and the higher education institutions in the organisation's environment.

\subsubsection{Betwixt and between: Contractors}

When individuals on a temporary contract enter the main office, their position as oppositional to that of permanent employees is marked by their different badge and act of signing in to the visitor book each morning. While contractors largely support teams by performing administrative and documen- 
tation work, in some teams they also perform tasks that are identical to those performed by permanent employees. However, contractors are not assigned to jobs that involve direct client or freelancer interaction; hence, they do not represent the organisation to the environment. Also, when interacting internally, their email address reveals their status through the word "consultant" before Advice Company's name: name@consultant.advice-company.com.

I had an opportunity to discuss the potentially conflicting situation of the contractors in more depth when Parul told me to make sure I would be around for her farewell cake-cutting ceremony in a week's time. I had accompanied her for several days and we had often met for after-lunch walks in the car park of the office building. From our conversations, I knew that she had been at Advice Company as a temporary employee with the "Consultant" badge for more than three years, and that her journey had been a layaway from one contract extension to the next, on an annual or even 8-month basis. Parul had often voiced her desire to be taken "on payroll" with her manager and had even been given the prospect of future employment a year ago. But as it became clear that the only option that would be offered to her was yet another temporary contract, she decided to apply for a job in another organisation. When I met her for a last interview three days before she left, the first thing she mentioned was how much she had appreciated the work environment at Advice Company. Parul explicitly expressed her feeling of attachment to a group of colleagues, whom she saw as close friends and whom she would not have considered leaving if it had not been necessary, in her opinion, to "play those politics". She had informed several managers that she would be more than happy to return to the organisation if an opportunity for a job on payroll arose. She said that she liked her job, but also that she saw herself in a client consulting role in the near future. She had sufficient experience and therefore needed to get out of her temporary contractor status. For Parul, being a contractor meant not only a major loss of benefits (such as paid health insurance), but also a lack of appreciation, with poor prospects for career advancement. Parul told me that she earned half of what her permanently employed colleagues earned for the same work. Furthermore, she had not received any incremental raise in her payment package for the past year and a half, although she felt she had worked "harder and longer than many others in this organisation". In the department in which she had worked for the past 15 months, several new hires had joined the team, all as regular employees. Witnessing others achieve the status she had desired for three years had been simply too much for her. 
Parul saw herself as a contractor insofar as she had temporary employment status, constituting a "transitory" situation that she hoped would lead to a permanent position in the organisation (Garsten 1999: 603). This hope was kept alive by several extensions of her contract and examples of colleagues with a similar contract status who had made it on payroll as permanent employees. She felt she had performed her job with at least the same commitment and ambition as her permanently employed colleagues. Despite not having the secure status of a permanent role and full membership to the organisation, she was convinced she had shown full responsibility for the tasks that had been assigned to her, often working way beyond official office hours. The lack of a "structural bond created by a regular employment position", while temporary employees are at the same time "drawn into extended circles of loyalty", makes "temporary employees share some of the inter-structural and ambiguous characteristics of liminality" (ibid.: 602), in Victor Turner's sense of "betwixt and between" social structures (Turner 1964). While Turner's concept of liminality assumes the individual as the central entity, the application of Luhmann's Systems Theory provides an additional view of the contractor's role. As systems consist of communication and not persons, individuals cannot be positioned inside or outside a system. Thus, individual contractors do not belong to Advice Company, but their communications do. Their daily communications with Advice Company employees in various functions are aspects of the social system organisation. Yet the description of these types of membership communicate the contractor role as one that is external to the organisation. This contradicting circumstance might provide an alternative model for understanding the conflicting situation, as perceived by Parul and other contractors. The next section deals with organisational membership only seemingly a more clearly defined category.

\subsection{Organisational membership}

Thus far, Chapter 4 has carved out the way in which Advice Company demarcates its boundaries with the environment, first with respect to the differentiation strategies in access procedures at the main office and, second, with respect to interactions with other systems in the environment. This section deals with organisational membership. Starting with a review of the various routes to becoming an Advice Company employee, I will then examine the way in which the rules of maintaining membership are communicated in the "new 
joiners' training" programme and, finally, discuss the "good" and "bad" ways of leaving the organisation, which are closely connected to re-entry options.

\subsubsection{How to become an employee}

There are several pathways to becoming an Advice Company employee, all of which are partially dependent on the status or skills required for a given job opportunity. But all of the established options have one aspect in common: they each refer to previously established connections with Advice Company, whether through an official organisational context or an informal social system.

The campus recruitment process (as described in Section 4.2.3) represents the most frequent route taken to secure employment in entry-level jobs, and this process stems from established partnerships between Advice Company and universities. The other common method for joining the organisation is via referral - an established praxis for recruiting employees into more senior positions. Those who do not join the organisation via campus recruitment usually have a (sometimes distant) relative, friend or neighbour working for Advice Company who is able to inform them of available positions within the organisation. The Advice Company employee passes the résumé of the interested acquaintance to colleagues in the organisation who manage organisational membership (i.e. those in the HR department). If the résumé is evaluated positively, the person is invited to take the company's standardised entry test; if this is passed successfully, the test is followed by recruitment interviews.

In addition to my asking my interlocutors about their path to employment in the interviews, I also observed the early stages of the rather informal referral process. When I accompanied Amjad in the city office, he was called on his mobile by a distant cousin whom he had met in person only a few times. The cousin was about to complete her studies and was interested in an entrylevel job with Advice Company; she wondered if he could help her with this. Amjad asked the young woman about her interests in the business field, any previous work experience and when she would be able to begin work. He gave her his email address and asked her to send him her résumé. He told her that either he would get back to her or she would be contacted by someone else in the organisation. The entire call took fewer than five minutes. As soon as he was off the phone, he walked to a colleague whose team had an open position and talked with her about the potential applicant. They quickly agreed that he 
would forward the résumé to her as soon as he received it and that she would take care of the next steps.

Referrals also describe cases in which a senior manager joins Advice Company and brings a number of employees from the previous organisation with him or her. Whether the referral is initiated on the basis of family ties, a fellow student or a previous co-worker, there are two prerequisites for this mechanism to function. First, both the referring and the referred parties must be members of the same social system in the Advice Company environment. Second, the referring individual must hold a position in the organisation that enables him or her to learn about open positions and establish a communication channel between the referred party and the relevant function in the organisation.

Finally, a person may join the organisation as a permanent member from a temporary contract or freelancer position. This route is also based on an existing relationship with the organisation. The option of re-entering as a former employee is not unusual, yet it is associated with a certain ambivalence, as discussed in the final section of this chapter (4.3.4). My interlocutors considered Advice Company not only the most prestigious organisation in its sector of the professional services industry, but also a gateway to attractive jobs in other companies. Advice Company employment was therefore often described as a stepping stone once an employee had "served" at least one year. Thus, becoming a member of the "Advice Company family" was rated as quite attractive and desirable. The elaborate entry procedures for non-employees mirror this self-observation of the organisational system, as does the multistage employee selection process. Once a contract is signed, a new employee takes part in extensive on-boarding activities through several induction sessions and a two-day training programme, which is the focus of the following section.

\subsubsection{Access training: Learning the rules}

In an attempt to understand how the organisation communicated its common values and rules of conduct, a good starting point was for me to attend the training for new employees. The "Discover Advice Company" training, as it was officially called, was held every few months, depending on the number of new joiners. It was organised by the HR department, whose members played several active roles in the agenda over the two days. All employees who had 
joined since the previous training were invited to the training from all three offices across the city, irrespective of their job function.

I was glad to also receive an invitation for the Discover Advice Company training via email in my second week of fieldwork. The invitation contained a detailed programme of the activity sessions and presentations. The location for the training was the largest meeting room in the main office, with space for 40 people. About 25 new joiners were already sitting in the room when the first speaker entered and began the introductory session. The speaker mentioned that he had been with the company for eight years and that he held a senior management position, and he asked the attendees to individually announce their name, department and length of time at the company. Most of the departments' names, which were presented in acronyms, did not inform me of much at that early point of my research; nor did they inform most of the attendees around me, as I learned during the coffee break. Instead, the senior manager hosting this first session accompanied each person's statement with a comment such as: "very important for us", "rising team" or "have lots [of] challenging projects on their plate these days".

At the end of the introduction round, three young men entered the room and apologised for being late, claiming to have been based at a different office and to have underestimated the time it would take them to get here. With a smile, the manager asked them to tell a joke, sing or dance in front of the group as punishment for being late. The men laughed and sat down, but the manager made it clear that he was serious, and urged at least one of the three to give a short performance. Complete silence flooded the room and I noted wide-eyed looks expressing a mixture of bewilderment and anguish exchanged between the participants.

After a few seemingly endless seconds, one of the three men slowly rose from his chair, swallowed, took a big breath and sang a short classical song in Hindi with a firm, almost professionally trained voice. The other participants cheered and the young lady sitting next to me leaned over to say: "I'm so glad it didn't hit me, I would have fainted in front of all." During the applause, the atmosphere became more relaxed, yet it did not ease up completely for the entire session. During his talk, the manager continuously called upon attendees at random to answer questions about certain aspects of the organisation's business strategy. While some were able to respond confidently, others clearly felt uncomfortable, biting their pen or fingernails subconsciously when they did not know the answer. Feeling much more like a participant than an observer, I spent the rest of the talk fully concentrated on what I would an- 
swer if he called on me. To my relief, I was spared and after 45 minutes, the next speaker entered the room. This woman was from the HR team, and she led us through a cheerful getting-to-know-each-other game, after which we enjoyed a coffee break.

The other speakers at the training session were managers of different departments of Advice Company. But unlike the first manager, these managers primarily spoke without posing questions to the audience. While the length of their membership to the organisation varied from a few years to a decade, their presentations were remarkably similar. Most talks began by highlighting the speakers' many years in the company, contrasting this to the situation of the new joiners around them. An introduction to the specific purpose and traits of their departments would follow, before the focus would shift to the general characteristics of Advice Company and the relationship to the client. Advice Company was described as a "premium consulting company", giving "more meaning to the answers [to] clients' strategic questions than competitors". The last speaker on the first day claimed that "we deliver solutions that are simple for the clients to understand and have their ROI [return on investment] in mind", and "we have this certain arrogance that the best solutions just come at a premium". These talks provided insight into the organisation's self-description. The audience was comprised of new organisational members, who had yet to learn the organisational structures and the rules of conduct within it. By opening their speeches by highlighting their many years of organisational membership in conjunction with their senior positions, and contrasting this with the situation of the new starters at the bottom of the hierarchy, the managers emphasised the value of tradition. They highlighted that internal status and decision-making power could only be gained from time and experience within the organisation, as opposed to various career steps made via changes between organisations. The importance of tradition was iterated in later months through a celebration of the organisation's anniversary, which again stressed the image of a "premium" consulting service provider in differentiation to competitors, justifying Advice Company's higher service charges.

The new employees were also made aware of the magnitude of the organisation they now belonged to, which operated in more than 100 countries. The messages given in the speeches included: "we are huge", "you are now part of a big global team" and "we can reach all across the globe, you are not small, only India, we are global". Other guidance that was frequently given during those two days included calls for "boundaryless behaviour" in the office and 
"shar[ing] information openly and freely across the organisation". I will return to these points in Chapter 6, when analysing internal differentiation and boundaries. The quest for "boundaryless" behaviour, however, implied a perceived existence of internal boundaries that needed to be overcome through conscious effort; indeed, my interlocutors perceived these boundaries to exist. Based on these circumstances, I will discuss in Chapter 9 how these boundaries play a role in Advice Company's project-related planning processes.

The organisation's strong orientation towards client systems was characterised by the prompt to "live client centricity", though this instruction was never clearly defined. Case studies were presented to emphasise that "it is in the end all about what the client wants" and that it was important to "understand the needs of the client". These case studies had the same narrative structure as my interlocutors' narratives of their most remarkable projects in the interviews: they related to very complex project mandates and hinged on dramatic stories about how the projects seemed too large to realise in too little time, and how the initial reaction had been to push back on the client's demands but how the projects were ultimately realised and turned into major successes for Advice Company, the respective departments and - of course the happy clients. The clear essence of these case studies was subsumed in slogans such as: "You don't say no. We don't say no. Advice Company doesn't say no." Here - again - the continuous emphasis on client centricity as a guiding principle suggests that practices exist which are running against it without mentioning them explicitly.

In the course of my fieldwork I came across many similar narratives negotiating the meaning of client centricity, both in personal interviews and casual conversations, as well as in town hall meetings and project stories that described examples of client centricity and the perceived impossibility of saying "no". However, during the introduction training at the beginning of my fieldwork, I was only slowly starting to suspect the relevance of client centricity as the superior value in the organisation's selection processes - and of an opposing counter-current to it. I did not foresee at that time that the opacity of client centricity was potentially the most productive working misunderstanding in this organisation. I will show, throughout this book, that the lack of clarity about the precise meaning of client centricity made the value particularly productive for the successful operation of the organisation.

I got a first glimpse of this during the lunch break on the second day of training. I had only just sat down at a canteen table with a few other participants when Kashish, who was also attending the training and was part 
of the team I was accompanying in those weeks, approached me. He leaned over and told me in a low voice that everybody from the team would be going to a nearby restaurant for the farewell lunch of a colleague who was leaving the organisation the following day. Kashish had been sent to look for me and to convince me to come along. On the one hand, I beamed with delight that my participation in that team event had been considered relevant enough for Kashish to be sent across the entire office area to fetch me and for the opportunity to see the team interact outside of the office premises; on the other hand, I was a bit reluctant to join, as we would certainly be late for the beginning of the next scheduled presentation of the training. The public avengement of the latecomers was still present in my memories, and I was not keen to sing a song in front of all of the participants. When I raised my concern to Kashish, he smiled and said, "Don't worry, we will be fine" - so I went along with the team to a long, interesting and delicious lunch event.

When we returned to the office after a good two hours of great food and lively conversation, I stood in front of the meeting room with Kashish and hesitated to enter. I must have given him a worried look, as he mumbled before opening the door: "Don't say anything, just look serious and take your seat in the room, I will take care of it." So we entered and received a very sharp "You're late!" comment from the manager holding the presentation. Kashish responded to my astonishment: "Sorry, but there was a very urgent high-priority client deliverable that had to be dealt with." Without another word, he sat down. The manager briefly nodded and continued with his talk. A few moments later, I glanced over at Kashish, who returned my look with one lifted eyebrow and a subtle hint of a smile. Obviously he had understood very quickly how to successfully play on "client centricity" in this organisation he had learned "the 'office talk', the rules of the organisation" (Krause-Jensen 2013: 44). In contrast to the three young men who had arrived late the day before, Kashish had explained the delay in a way the organisation could process; therefore, we got away with it.

\subsubsection{Maintaining membership: The grade-based ranking system}

The final session of the training was held by a senior HR representative, who played a professionally produced 5-minute video clip featuring the well-known top managers of the organisation welcoming the new joiners. He then explained how the new joiners could ensure that they stayed in the organisation, referring to the performance measurement scale along 
which each employee would receive a rating by his or her manager once per year. This ranking would be based on objectives that would be agreed at the beginning of each financial year between the employee and the manager, and would be given on a scale from 1 (worst) to 4 (best). This rating would not only determine the amount of the annual bonus payment, but also enable or disable promotions, which could only be granted to those receiving a rating of 4 . The HR representative furthermore stated:

Employees rated only with level 1 are asked to move out of the system immediately as well as the lower level 2 performers. They are obviously not living up to our values. Only level 3 and 4 performers are to be retained in the organisation, and only with a rating of level 4 you can apply for a different department or for the mobility programme once you have been for at least two years in your initial department.

The mobility programme would allow employees to gain placement in one of Advice Company's overseas offices. This grade-based system illustrates the organisation's perception of objective and quantitatively measured conditioned membership, mirroring the joining process by requiring a graded entry test as a pre-condition for invitation to an interview. However, the "annual performance review" frequently raised several - often bitter - discussions about differing perceptions over the right, "justified" grade between a team member and his or her manager. But no matter how controversial a certain grade might be, all subsequent decisions built on this grade; for this reason, an insufficient grade could lead to a denied application for placement in a different department or promotion.

\subsubsection{Leaving and re-entry}

Similar to the various routes of becoming a member of the organisation, several paths lead out of it. On the basis of the grading concept discussed above, one reason an employee might leave the organisation is that he or she will "have been asked to leave". This is the "organisational euphemism" (KrauseJensen 2013: 51) for being fired due to poor performance. This pathway, which is primarily based on the organisation's decision to discontinue a specific employee's membership, was almost impossible for me to gain insight to. Unlike employees who left the organisation on the basis of other decision routes, those who were asked to leave did not return and were rarely mentioned to me as individual cases. They existed as a threatening category that one might 
have "heard of". Another pathway to exit with a similarly low likeliness of return is taken by young female employees who decide to drop out of the workforce after marriage or motherhood in order to focus on the needs of their family. The extent to which these decisions are driven by individual selection or extrinsic decisions from the family environment vary from case to case; thus, an assessment of this pathway is not included in this work. ${ }^{2}$

The other two pathways for discontinuing organisational membership are based on employees' selection decisions to accept an attractive job offer from a competitor or a client or to continue one's studies. "Putting down the papers" (as the employees call the process of resigning) for a better paying job and/or a career jump is common. This decision is often combined with the hope that one's career will be boosted. A common strategy associated with this step is to re-enter Advice Company after a few years at a higher job level than one would have been able to achieve by remaining in the company for that time. This type of "career plan" is not supported by the top management, as an executive board member said: "I don't want my staff to think there is a shortcut to a career." Nonetheless, I accompanied a number of colleagues who had taken this route. Similarly, I also came across examples of people "who jumped the queue" through this method.

This trend can be connected to the phenomenon that employees who leave the organisation often remain in contact with their former colleagues via organisation-independent communication channels such as Facebook or the team's WhatsApp group. Ex-colleagues frequently take part in their previous team's after-hour activities, meet their previous colleagues for coffee and attend the weddings of their former teammates. During the pilot study in March 2013, I accompanied a young man who, by the time I began official fieldwork four months later, had left the organisation. At that point, his former colleagues knew about his professional life at a competitor's organisation and his honeymoon trip. His name was still mentioned at the team morning coffees. When I accompanied the colleagues of that particular team to a teammate's wedding reception, I was therefore not surprised to meet the young man again. Similarly, he had been informed of my return to Advice Company for fieldwork and the several activities I had attended with his former colleagues. We went together on stage as a group to pay respect to the bridal and parental status on the career persistence of women software professionals in India. 
couple and the ex-colleague stood amongst us when we got our official (and obligatory) group picture taken. Also, when we later posed for less formal pictures and had dinner, he remained with the group and there was no difference between him and the current team members. He was still a member of the group of "groom's workmates".

The client consultant whose farewell lunch I "escaped" to with Kashish during the new joiners training, also remained in touch after her exit from the organisation. I met her several times for an after-work coffee together with another ex-colleague of hers. Her ties with her former teammates remained quite active, as she had started to share a flat with a former teammate a few months after leaving. Even eight months after her exit from the organisation, she showed me several online conversations she had recently had with her former managers at Advice Company. Through all of these channels, she had remained well informed of the team situation and the latest Advice Company gossip. Professionally, she worked in the environment of Advice Company as a competitor, and thus battled with her former teammates for clients' projects. While she remained in contact with her former colleagues, she had not returned to Advice Company as she had initially intended; instead, she had moved to the client side. Despite being a strategy not approved by management, returning to the company after a taking a job elsewhere seemed common. When I accompanied client consultant Gopal, who had just re-entered the organisation after an absence of 18 months, he expressed a feeling of having an obligation to return:

When I resigned, it was clear between my manager and me that I [was] only leaving under the condition to be back here the moment he need[ed] me. While I stayed in touch with the teammates on casual stuff, I did not even speak a single word with him [the manager] during these months. But then I saw his name on the display of my mobile when he called. I picked up and just asked by when he want[ed] me to be back, that was it.

Staff turnover in business organisations in India has been broadly covered in research (Khandelwal 2009). However, the strategy of leaving an organisation with the intention of later re-entering seems to have been less explored. Individuals who have left Advice Company to work for a competitor and later reentered Advice Company at a higher position are critically labelled by management as those intending to take a "shortcut to their career". In contrast to those who re-enter from a competing organisation, "good" returners are those who leave the organisation to continue their studies (e.g. by pursuing an MBA 
or $\mathrm{PhD}$ ) and return at a higher job level because they have earned a more advanced degree. While the perception of re-entry under these circumstances is different, the communication pathways are similar to those described above. In either case, ex-employees make their re-entry to Advice Company via their ties with former teammates. So, despite having left the organisation, their communication with the organisation remains active and the social sub-system continues to function. This makes re-entry realistic, should an opportunity or a perceived obligation to return arise. Gopal captured this notion of a social system emerging beyond the organisational boundaries in the paradoxical phrase: "We might be gone, but still there."

\subsection{Concluding remarks: Operative closure and openness to the environment}

Luhmann's proposition of self-referentially closed systems with interactional openness to the environment "contradicts the classical opposition of closed and open systems" (Luhmann 1995a: 37). In Luhmann's understanding, a system must be operationally closed in order to interact with the environment, otherwise the system-environment difference will cease to exist. This makes the idea of a system boundary with permeable qualities misleading. A system boundary cannot be understood as firmly established and then becoming pervious, but must be understood as a social construct that exists through its communication. When the boundary is not communicated, it disappears. The communication hence acts to define a system's boundary and what is deemed pertinent to it.

I have illustrated how Advice Company's boundaries are, on the one hand, sharply communicated through sophisticated acts of differentiation via elaborate access procedures, outgoing communication control to clients, multilevel recruitment procedures and strict rules of organisational membership. But on the other hand, the boundaries are furthermore maintained through daily interaction with the environment, such as that with freelancers, who are provided a dedicated working area in the street office. This space denotes openness to the environment, yet clearly communicates organisational boundaries.

In contrast to these dividing lines stands the relationship with contractors, who work alongside permanent employees in identical job profiles; therefore, their work clearly belongs to the social system of Advice Company. 
When employees and contractors communicate on project-related topics, the organisational boundary is not communicated and hence does not exist: employees and contractors comprise a social system. Only in situations such as contract renewal or the daily sign-in procedure is the boundary communicated and therefore brought to existence. Second, the established campus recruitment partnerships with universities enable Advice Company to conduct recruitment sessions on campus; in return, universities market this partnership as a selling point for their programmes. This relationship can be viewed as a structural coupling of interdependent systems. Universities and Advice Company collaborate effectively on the basis of wellestablished conventions, along which boundaries emerge (or not). I have also described how employees who voluntarily leave Advice Company maintain over months and years - frequent multi-channel communication with their former colleagues. While this phenomenon might be inevitable in any highly specialised business community, it shows that ex-employees remain a part of a social system that has been initiated in the context of Advice Company. Similarly, continued participation in after-office activities, wedding receptions and the like demonstrates the way in which employees communicate with each other (or with ex-colleagues) outside of the direct work context and illustrates that social sub-systems can emerge beyond the organisational boundaries from which they originate.

The cases presented in this chapter have illustrated how the conventional understanding of organisational boundaries can be challenged by the application of Luhmann's theoretical propositions. At the same time, I have shown how the organisation establishes its differentiation to the environment. With this differentiation as a foundation, further environmental differentiation is carried out through, for example, strategies of aggregation for relevant systems in the environment (Luhmann 1995a: 187). Advice Company differentiates its environment into clients, freelancers, universities and contractors. Generally cleaning personnel, office equipment providers, landlords and other supplying systems would have to be included here. But they are not concerned directly with the business process and are therefore insignificant for this work. The following chapter analyses the internal differentiation of Advice Company by looking more closely at the three offices and their respective relationships to the organisational structure. While a first glance might suggest a clear picture of these relationships, I will present a number of contradictions that will distort attempts at an easy answer. 


\section{Internal Differentiation: The Offices}

Chapter 4 focused on the organisation's differentiation to the environment and its differentiation of the environment. The next step of the analysis is to trace the strategies of internal differentiation and the emergence of subsystems within Advice Company. With this aim, Chapter 5 characterises the three offices of Advice Company in the city and describes their meta-function in the organisational structure. The purpose of this chapter is hence to build another layer of understanding of the organisation as a social system. Chapter 6 builds on this differentiation and zooms in on the internal differentiation within the individual offices by tracing the perceived invisible boundaries between departments and teams.

Commencing with an introduction to the internal differentiation of social systems more broadly, the chapter focuses on the role played by the three offices in system differentiation. On the basis of a comparison of each office's access procedures (Section 5.2) and equipment (Section 5.3), the three offices seem to be located on a continuum representing distance to the client, with a hierarchical structure suggesting client centricity as the primary value along which Advice Company orientates. The analysis of the atmosphere as perceived by the employees across the three offices (as presented in Section 5.4), however, indicates a more complex internal differentiation strategy. The analysis reinforces the theoretical framework of Gernot Böhme (1995), who understands "atmospheres as tempered spaces". The ethnographic data illustrates how the client-centric internal differentiation is recreated in the office atmosphere. But at the same time, the analytical category of "atmosphere" allows for a differentiated view of the offices with contradicting notions that suggest ground reality as an opposing value to client centricity and hence the second term of Advice Company's guiding difference. 


\subsection{Increasing differentiation to reduce complexity}

Internal differentiation is a key trait of any social system. Internal subsystems replicate organisational boundaries with the environment and can therefore use these boundaries to establish a special environment for their operations. Within this "secured" organisational setting, the sub-systems serve as environments to each other. On the one hand, this increases the complexity of the entire organisational system; on the other hand, it reduces the complexity of the sub-system: the sub-system only needs to be concerned with autopoietic reproduction and boundary differentiation to its own system-specific environment, as the reproduction of the whole system is already taken care of. Luhmann describes this reduction of complexity as follows:

Internal differentiation connects onto the boundaries of the already-differentiated system and treats the bounded domain as a special environment in which further systems can be formed. This internal environment exhibits special complexity reductions, which are secured by the external boundaries; relative to the external world, it is an already-domesticated, alreadypacified environment with lessened complexity. (Luhmann 1995a: 189)

While differentiation occurs constantly within an organisation, only a limited number of differentiation forms persist to the extent that they constitute subsystems (ibid.: 190). Luhmann distinguishes the persistent forms of differentiation into five categories:

[D]ifferentiation into similar units (segmentation), the differentiation of center/periphery, the differentiation conforming/deviant (official/unofficial, formal/informal), hierarchical differentiation, and functional differentiation. Apparently, the only forms of differentiation able to survive are those that can mobilise processes of deviation-amplification (positive feedback) to their own advantage and keep themselves from being levelled out again. (ibid.: 190)

Besides listing functional and hierarchical sub-systems, Luhmann also describes sub-systems based on differentiation as centre/periphery or conform/deviant. The segmentation of a system into equal units, which is typical for clans, is less prevalent in the organisational context. According to Luhmann, most emerging sub-systems in an organisation - such as discussion groups that come together in an aisle or around a desk - are of short duration and can only be regarded as interaction systems (ibid.: 193). 
There are several understandings of the notion of a centre/periphery distinction in the context of organisational structure. One point of view stems from network structure theory and understands the "core" as a cohesive group of core actors, in opposition to peripheral actors, who are loosely connected in their network ties to the core group. Such structures are investigated through social network analysis (e.g. Mintz and Schwartz 1981, Borgatti and Everett 2000). Another approach to the centre/periphery distinction is based on the type of membership to an organisation, whereby permanent, highly skilled employees are considered the core, with their knowledge and skill considered of high value to the organisation and worth securing through membership privileges. In this view, the peripheral workforce is the group of employees on temporary contracts, whose expertise can be bought when needed and who are thought to have a loose relationship with the organisation (Deery and Jago 2002: 342). Recent studies have argued that this bifurcation of the centre/periphery structure is not necessarily valid, as more complex layers of internal workforce differentiation exist, and employees on temporary contracts do not necessarily have higher job changing rates than their permanently employed peers. Gino Cattani and Simone Ferrarini, for example, argue that "peripheral actors are more likely to con tribute fresh perspective to the system and maintain high intrinsic motivations, although they lack the visibility and endorsement necessary to boost their work's recognition" (2008: 827).

An example of an anthropological study in the organisational context that adopts the analytical framework of centre/periphery is Christina Garsten's (1994) ethnography, Apple World. Her work investigates the cultural similarities and differences within the three interlinked offices of the technology company Apple. Her field sites include the organisational headquarters in California, the European head office in Paris and the Stockholm city office; along these offices, she traces centre/periphery relationships in conjunction with characteristics of inclusion and exclusion. Furthermore, her work analyses the way in which the concepts and strategies developed at the organisational headquarters are reinterpreted and undergo additions and modifications across the organisation.

Luhmann's distinction of centre/periphery suggests a geographical slope in a hierarchical order; this differentiation might be suitable for a client-centric organisation such as Advice Company. Yet such a differentiation might limit the perspectives one can take on internal differentiation. Therefore, in this chapter, my analysis will focus on the extent to which Advice Company's internal structure repeats the system-environment differentiation based on 
the guiding difference client centricity / ground reality. To provide insight into this first aspect of the black box of organisational functioning, I start with an analysis of the differential access procedures across the three offices.

\subsection{Access procedures: From elaborate to basic}

As illustrated in Section 4.1.1, access to the main office is granted only when one passes three gates that gradually differentiate access based on one's formal relationship to the organisation. Both the elaborate access procedures and the type of office building at the main office provide insight into the organisation's internal differentiation relative to the other two offices of Advice Company in the city. The names I have given the three offices are partially terms used by my interlocutors. There was no consistent nomenclature for the three offices, but the most broadly used terms referred to the names of the districts in the city in which the different offices were located. Another naming strategy was to refer to the acronyms of the main department or function at the location, such as the "TFA office" (fictional acronym). A third strategy named the office in reference to the company operating or renting the building. All the names derived from those three strategies might have threatened to accidently disclose the identity of Advice Company. Therefore I have decided to take "main office" and "city office" as names for the first two offices. These are both terms which were used by my interlocutors, albeit not as often as the first naming strategy I described. "Street office" is a term I have given to the third office, as all local terms were not sufficiently confidential.

\subsubsection{The city office}

Advice Company's second-largest office, with about 250 employees, is located in the centre of the city in a compound of several office buildings in which various organisations are based. The compound is significantly smaller than the one that hosts the main office and there are two options for entering the compound: one can enter from the main street as a pedestrian or via the alternative entry from a side road, through which it is easier to avoid the heavy traffic and to reach the employee parking lot. At both entries sits a security guard, who checks individuals for a badge issued by one of the companies onsite. Advice Company's office is located in one of the four flat buildings on the compound and previously served as the main office several years ago, before 
the main office moved to its current location. This location history will become relevant in section 5.4, in my analysis of the perceived office atmospheres. There, I will show how the memories of the main office employees of their former workplace play a major role in shaping their image of the current city office.

After passing one of these first gates, one must walk 50 or 100 metres, respectively, to the Advice Company office entrance. A small placard of about $40 \times 20$ centimetres indicates the company name next to the sliding doors, which lead into the reception area - a room of approximately $5 \times 12$ metres. In the reception area, white tiles line the floor and the walls are faced with dark brown wooden panels garnished with white, illuminated plates on which posters of the latest global Advice Company internal image campaigns are displayed: "Commit to Grow" or "Strive for Big Impact" are written next to images of smartly dressed 20-somethings from all ethnic origins. Behind a dark brown wooden counter with a glass top featuring a small fresh flower decoration sits a man wearing a uniform of a white shirt and a police-style hat. The counter has a small hip-level annex to the left, on which a guest book is left open. Next to the counter is a seating area with two black leather sofas and a chair arranged around a low table. On both ends of the reception area, doors lead to the actual workspace, which is - similar to the set-up of the main office - invisible from the reception. The neat appearance of the desk, with the illuminated posters, is disrupted by a blue, cheap looking plastic rack that is overloaded with papers and a standing fan on the right side.

When I came to this office for the first time I accompanied Poorva, who was primarily located in the main office but travelled to the city office regularly for meetings with the staff there, for whom she was the main HR contact. Hence, my entry was probably easier than what a completely new person would have experienced. But even taking this into account, the access procedure was much simpler than the procedure in place for accessing the main office. Poorva and I arrived by taxi at the side street entry of the compound and she told me to take out the "Consultant" badge I had been given by the main office's receptionist. With this in hand, I passed through the gate and hardly caught a glance from the two security guards who were standing around a small table in the booth next to the barrier. When we reached the office building and entered through the sliding doors into the reception area, the watchman smiled at us and opened a drawer to fetch me a "Visitor" badge before my patron for the day could tell him: "Frauke is our intern at the main office and she will come here for the next three months, okay?" He smiled 
again at me, passed the badge over the counter and told me to return it in the evening. Then I signed in to the visitor's book and Poorva and I swiped our badges on the card reader to open the surprisingly narrow door into the office area.

Over the next weeks, I happened to arrive several times together with Aranjit, a colleague I had accompanied at the main office before. From this I learned that there is indeed no distinction made between the type of visitor arriving at the city office. Both Aranjit and I had to register by writing into the very same book and we both received the same "Visitors" badge to pass through the doors. Furthermore, in this office, swiping the badge served solely to open the door and not to track entry and exit. When colleagues went for chai breaks at the stall on the street, they would ask around to make sure at least one person in their group had a badge to open the door for the others when they returned. In contrast to the main office, in this office, the doors are unlocked from the inside by pressing a button rather than swiping a badge. It is very apparent that this access procedure is significantly less complex and distinctive than the procedure in place at the main office: not only are there fewer gates to pass through and a reception desk guarded by only one person (instead of two), but no distinction in badge is made between the employees located in this office and everybody else, regardless of their relationship with the organisation.

The symbolic rather than primary functional meaning of this procedure is marked by the fact that, during a few weeks when I had to arrive at the office very early (at 7.30am), I found "my" visitor badge (I always got one with the number 6 written on the back) lying on the counter on top of the visitor book next to the pen. Thus, I was able to grant myself access to the office without having to wait for the uniformed receptionist to return from checking on the cleaning staff.

\subsubsection{The street office}

The third and smallest office of Advice Company was only six kilometres away from my home, yet it took me almost 90 minutes to travel there by rickshaw due to the heavily trafficked roads of the morning rush hour. On my first day at the office, there was no opportunity for me to accompany a colleague. However, my interlocutors at the main office had given me instructions on finding the office location. Once the rickshaw driver dropped me off at the 
landmark building ${ }^{1}$, I walked up and down the road a few times looking for the office. Just when I was starting to feel lost and slightly uncomfortable, I finally recognised a building that corresponded with the description I had been given and eventually spotted the small sign with Advice Company's name on it. Despite the directions and description I had been given, my imagination did not match what I saw: a small, two-storey brick building with a veranda and a one-metre high wall separating the property from the street. A big sign informed potentially willing investors of the planned reconstruction of the building; indeed, as I learned later, a department was scheduled to move to a different location in the next year. When I arrived at the veranda, a motorcycle was parked at the building's wall and three men in shirts and business trousers were gathered for a cigarette break. When I approached the group and asked for entry, they looked seemingly irritated and one reacted by questioning with whom I had come. I later learned that a farlang (foreigner) was not such a rare species in the office, but an unaccompanied (i.e. uncontrolled) one was absolutely uncommon. Hence, the situation required informal access control, as exhibited through the colleagues' attentive questions. When I informed them that I was on my own and named the contact person I had been emailing over the past days, one of the men hesitantly pointed to the open door to the right, through which I entered.

Once my eyes adjusted to the sudden gloominess, I detected a hallway and a small desk at which a security guard sat, beneath a narrow staircase leading to the upper level. Before I could explain my presence to the watchman, one of the men leaned his head through the doorway and said a few words in Marathi, of which I only caught the name of my contact person. I imagine he said something like "She's here to see Rahesh", as the watchman reached out with his right arm and pushed open a door without a handle, indicating that I could enter. With another step downwards through the doorway, I found myself standing in the office area directly in front of Rahesh's desk. Rahesh greeted me and introduced me to his team.

In contrast to the previously described offices, this third one does not have a formal access procedure for entry to the office area, and employees only wear their badges when visiting one of the other two offices. The differentiation of this office is not marked by access procedures and visible distinctions such as different types of badges. Rather it is exhibited by the bisection of territorial 
space: the ground level is dedicated to Advice Company's employees while the upstairs provides the work area for freelancers. There are no signs to indicate these territories, but they are distinct in their differences in equipment, and the boundaries are reiterated several times per day. An example of this was given in Chapter 4, Section 4.2.2, when a freelancer's entry into the Advice Company's employee area was perceived by a manager as a border infringement, and strongly communicated as such.

\subsubsection{The production of differentiation through access procedures}

When comparing access procedures across the three offices, it becomes apparent that the main office is not only characterised by its role as the functional headquarters of Advice Company in India (by hosting the top management positions and the largest number of employees across the largest physical space). In addition, the elaborate entry procedures imply that the office is the most client-centric of the Advice Company India offices and that it is an achievement for a person to reach here. This is where contact with clients is managed and physically takes place, and where people are "corporate" and "strategic", as described by their colleagues in other offices (see Section 5.4.1). It might be a coincidence that the main office is located on the sixth floor of the building - several floors higher than the city and the street office, and for many employees, representing countless steps up the career ladder. The office is seen as a place of career advancement - a place where "client interaction happens" and a place where employees can "directly influence projects" and do not have to sit "handcuffed behind the wall", as remarked by Sameer, an employee at the city office (see Section 10.5). The main office also stands in contrast to the street office - the two-storey brick building and the site associated with the "ground reality". The street office hosts the "simple people" those who "prefer to be with my freelance people and not the big bosses there", as stated by Rohan, who was located at this office. While not everybody in the main office is a top manager and/or in direct contact with clients, the teams in the other two offices, by definition, have a supportive downstream function that is further away from the client, and hence less prestigious within the client-centric organisation. 


\subsection{Inside the offices: Differences in space and equipment}

This internal differentiation of the three offices along the continuum of client centricity is not only manifested in the access procedures, but also in the different physical characteristics of the offices.

\subsubsection{Inside the main office}

When the frosted glass door at the reception opens - following the "beep" of the card reader panel - one passes along a 20-metre-long walkway from which doors open into a main meeting room with a capacity of 50 people. On the left side, an A2-sized poster announces the 30 winners of the annual performance awards, with pictures, names, designations and the names of the awards won. These awards feature in the annual performance grading system (see Chapter 4, Section 4.3.3), and nominations are made at a town hall meeting at the main office. To be nominated for an award, one must receive a grade of "outstanding performance" in one's job, and this performance is often validated by several project-related awards that are awarded over the course of the year. At the end of the walkway, the office area emerges with its stunning 180-degree view of the surrounding commercial and residential buildings.

Rows of desks of the same size and style stretch across the carpeted floor, interjected periodically with semi-open chat corners or glass-walled meeting rooms that serve as interactive workspaces. The 461 individually assigned desks are about 120 centimetres wide with 50-centimetre-high walls between them; they mostly feature a fixed monitor, a landline phone and a drawer, which can be locked. The office chairs are also identical and are only sometimes individually marked by an employee's jumper slung over the back or a small cushion on the seat. The desk partition walls allow for individualisation, and employees decorate these with objects belonging to the official organisational context, such as lists of phone numbers or laminated award cards that have been granted by the management team. The desks are, however, also spaces for personal objects and images. Apart from displaying family photos, the employees decorate their desks with decorative objects, cartoons and small figures and images with religious connotations, such as Ganesha miniatures or Catholic rosaries. This latter category of objects not only individualises the desk space but also sends potentially alternative or even competing 
messages to official corporate communications (Gavin 2015: 97, Kostera 1997: 174).

A team of women and men in the distinct dress of cleaning workers not only ensure that the two machines serving complimentary coffee and the water dispensers remain clean and well stocked, but they also collect office chairs that are left in aisles and neatly place them back in front of the desks. In addition to the meeting rooms and chat corners, two seating areas with comfortable sofas and chairs are available for informal or spontaneous conversations. The canteen, with 100 seats, serves daily (between 9.00am and $7.00 \mathrm{pm}$ ) a range of local meals, freshly prepared chai, fruit plates and sandwiches. Instant noodle dishes known as Maggi (although not necessarily from that brand) are extremely popular amongst the employees as an evening snack around 6.30pm.

The seven flat television screens that line the outer walls of the office display Advice Company's corporate videos and cricket matches. Representing a contrast between work and leisure contexts, the screen in the canteen shows music video clips of the latest Bollywood blockbusters, all on mute. The office has two strategically placed washrooms: one - as already mentioned directly in front of the reception area and the other right next to the canteen. The latter washroom is placed just past the dirty dishes return rack, so that employees can conveniently wash their hands after lunch. This is standard procedure, as most lunch dishes, consisting of vegetables/meat and chapatis (wheat-based pitas), are eaten with the right hand. A firm iron door leads to the "recreation area" - a seemingly unfinished hall that can be turned shortterm into a desk area, if required. However, the room is mostly known for hosting a popular table tennis table. After accompanying several colleagues from the Muslim community to this room, I discovered that the office also uses it as a prayer room. At the main office slightly less than $40 \%$ of the employees are women and I was told by an HR colleague that this ratio remained fairly stable over the past two years.

\subsubsection{Inside the city office}

When I first entered the narrow hallway of the city office I was slightly surprised by the smooth and corporate touch that the office radiated, as the stories the main office colleagues told me about the "fun atmosphere" had prepared me to expect a different, less formal, setting (see Section 5.4.2). In this office, a wide, almost windowless hallway leads to several office rooms and a 
recreational area equipped with an air hockey table. The other office rooms along the hallway lead to sub-team offices, the main meeting room and the office of the department manager. At the end of the narrow hallway is the central office, with its 10-metre high ceiling. Although the location does not hide its origins as a production site, the efforts to turn it into a corporate office have been effective: the walls are painted white, the tin ceiling is well maintained and three huge posters with Advice Company's logo decorate a massive wall. The main desk area, with 144 seats, is lit by fluorescent lights. Opposite the entrance are meeting rooms and a cafeteria with windows. An open stairway from the desk area leads to a mezzanine in the form of a gallery.

The main areas on both levels of this office have desks placed perpendicularly, forming bays of four. Individual workspaces are created by glass dividers and low walls. Similar to the main office, in this office, each desk has a landline phone and a drawer. Arranged at the side of the main area are small bays separated by shoulder-high partitions, each with a single desk and an extra chair. These provide spaces for team leads and group managers. The head of this entire department - the "Vice President", as Imran whispered when he walked by - sits in an office at the entrance. The building has two washrooms - one on the ground floor and a very small one on the upper level. The cafeteria has about 30 seats, provides complimentary water and coffee and offers breakfast and lunch options. A "wall of fame", while not as prominently located as in the main office, displays the quarter's top performers with their pictures, names and designations. These awards are distributed publicly (i.e. openly in the office), similar to the awards ceremony in the main office, each quarter.

The upper level of the city office is very similar to the ground floor. But the outside walls reveal the former structure of the building. The upper level must have once hosted smaller office cabins for middle managers, as the desk area is interjected by hip-level walls that probably once had glass windows.

While similar to the main office in terms of equipment, the main differentiation between this office and the main office, as perceived by the employees, is the "relaxed atmosphere" of the city office, which I discuss in Section 5.4. Yet this city office also carries an air of wear and tear, or abrasion, with its relics from a previous set-up and the fact that the entire office is a bit too large for the size of the teams that are permanently based there. This results in several empty desks and two temporarily re-located teams that belong to completely different business sections. The employees pointed out to me that basic operating equipment - such as phones or the Internet - could get disconnected 
during the heavy monsoon season, when the area outside the office would get flooded. Several years ago, this office hosted client-centric functions such as the organisation's decision-makers and client consulting teams. But that is no longer the case and, accordingly, both access procedures and equipment have been scaled down. Poorva, the HR representative for this office explained that the gender ratio at this location dropped in the last 18 months from over $30 \%$ to $25 \%$ of female employees. As one of the reasons she mentioned the increased demand for colleagues supporting the US offices and therefore having to work late in the evening.

\subsubsection{Inside the street office}

Located in a two-storey brick house of approximately 150 square metres on each level, the street office is characterised by a strict division between the ground floor and the upper level. The air conditioned ground floor features two office rooms with desk bays, computers and other office equipment such as printers, phones and office chairs. The two upstairs rooms are of comparable size, but equipped strikingly differently, with 40-centimetre wide plain tables set up along the walls, and white plastic chairs. Three phones and a printer complete the setting, together with a tiny room for meetings. There is no air conditioning and there are no individual workspaces. While the ground floor constitutes the office space for Advice Company's employees, the upstairs rooms comprise the work environment provided for freelancers. This special interaction space for the organisation with its environment is not, in itself, opposed to client centricity, as the meeting room facilities for clients have a similar function (see Chapter 4, Section 4.2.2).

The difference in the status of the street office - not only due to its freelancer area upstairs - becomes salient when one compares its Advice Company internal work area to that of the other two offices. The first striking aspect of this smallest office is the lack of space. The desks form five bays of six workspaces, with three desks on each side. There are a total of 30 workspaces, equipped with fixed flat screen monitors, a keypad on a drawer beneath the deep desk and a fixed computer tower on the floor. The bays are barely wide enough to accommodate two office chairs, back to back. When both opposing workspaces are in use, employees must take care that the backs of their office chairs do not permanently bump against each other. The neighbouring room provides another 20 very similar spaces. The desks are only partially assigned to individual colleagues, as they are meant to cater for multiple users. About 
one-third of the seats are shared amongst team members who are not permanently based in the office and only come in to quickly check their emails or to join a conference call. Landline phones are shared between three desks, which results in such devices being frequently passed along the desk row.

Team leads each have a dedicated, separate cubicle with their own desk and phone, but their desk spaces are frequently shared with team members who wish to join teleconferences or discuss projects. A small office with a door is reserved for the head of department, who is primarily located at the main office but sometimes works from this branch. There is no water dispenser or coffee machine and no space for lunch breaks at this office. At lunch time, small groups of two to five colleagues congregate at a desk and unpack their dabbah - a behaviour that would result in an immediate rebuke from the reception's security officer at the main office. A sink is available for employees to wash their hands after lunch and smoking is permitted on the terrace and in the backyard. The building has two washrooms, one on the ground floor and one upstairs, which seem to be separated by gender (with men on the ground level and ladies upstairs). But I was also told that the ground floor toilet is for managers and the upper floor toilet is for everyone else. The seemingly unresolved nature of this topic was reflected in my interlocutors' frequent suggestions that we go to an adjacent commercial area for a walk after lunch so they could use the washroom there. According to Deepika from the HR team only $15 \%$ of Advice Company's employees at this office are women, a number the organisation wished to increase.

The Internet connection was reportedly shaky and under direct threat during the monsoon season - a circumstance that regularly impeded communication with the other offices. However, this was not raised as a major concern. Instead, the employees frequently emphasised the practicality and suitability of the office for interacting with freelancers. The office was therefore positioned at the opposite end of the client centricity scale, with respect to access procedures and equipment.

\subsection{Atmospheres as "tempered spaces": Office perceptions}

As I illustrated in Sections 5.2 and 5.3, the internal differentiation of Advice Company's offices in the city goes beyond mere segmentation "into similar units" (Luhmann 1995a: 190). The three locations can be distinguished according to the guiding difference of client centricity on the basis of the complexity 
of their entry procedures and physical equipment. As a third dimension of the three office sub-systems, I propose a comparison of the different office atmospheres. This topic was brought up by the employees, themselves, not only when comparing the offices but also when describing their own workplace even without direct emphasising its differentiation to the other offices.

While atmosphere is a term that is frequently used in everyday language, its conceptualisation for ethnographic analysis constitutes a challenge due to the multi-sensory dimensions of perception. The motivation for the following analysis stems from an ethnographic case study on the atmosphere of a south Indian village looking at the interconnectedness between persons and environment (Heidemann 2018). Heidemann approached the "challenge of atmospheres"2 in ethnographic analysis by also drawing on the theoretical groundwork of Gernot Böhme, a scholar who is frequently cited in the anthropology of aesthetics. Böhme argues for a "new phenomenology" that expands the understanding of aesthetics from its sole relation to art and artworks to a general theory of perception. A central element of this is the analysis of the correlation between environmental characteristics (Umgebungsqualitäten) and the sensitivities of the people (Befindlichkeiten) in these environments. Atmosphere is the concept through which this correlation is manifested (Böhme 1995: 16). To Böhme, atmosphere is both a primary term and an object of study, and it refers to the relationship between the shared reality of the perceiver and the perceived:

The primary themes of sensuality are not the things that we perceive, but what one feels: Atmospheres. When stepping into a room I am tempered through this room in one or another way. Its atmosphere determines my own perception. Only when I have entered, so to speak, into the atmosphere, am I able to identify and discern an object. Atmospheres, how they can be sensed in relation to environments as well as to things or people, are the central theme of aesthetics. Aesthetics seeks to explore the relationship between the qualities of environments and sensitivities. It asks how certain, quite objectively ascertainable characteristics of environments can modify our condition within these environments. (Böhme 2013: 15, own translation) Hornung in October 2014: http://www.ethnologie.uni-muenchen.de/personen/profess orinnen/heidemann/workshop_atmospheres_2014.pdf. 
According to Böhme, atmosphere can only be pursued through experience: one must first be exposed to and affectively concerned with it. A room, for example, can have a cheerful or gloomy spirit, but this is not a subjective mood, as such an atmosphere is experienced as quasi-objective and refers to a common state of ego and its environment. In that sense, Böhme defines atmosphere as a "tempered space" (gestimmter Raum) that conveys certain sensitivities or vibes (Böhme 2001: 103).

Atmosphere exists in a definable space. It is dependent on the people who are present and experiencing the space with their senses activated by the atmosphere. It is also dependent on the objects present, and their characteristics. Atmospheres exist, they affect the people present and they are quasi-tangible - or at least describable (Rauh 2012: 25). Simone Egger applies the concept of atmosphere to dimensions of reception in urban space and illustrates, through the example of football arenas, how similarly built structures yield specific atmospheres, enriched by a historic depth of memories, achievements and defeats that form a "cumulative texture". In her example of the FC Barcelona football club, atmosphere is expressed through slogans such as "Más que un club! [More than a club!]" (Egger 2015: 160). Sebastian Uhrich also applies Böhme's concept to football arenas, yet from the other end, so to speak: his work is geared towards an event-marketing perspective and seeks to identify factors that are regarded relevant for purposively fabricating "good atmosphere" in a stadium (Uhrich 2008: : 69-71).

The application of Böhme's understanding of atmosphere to the context of an ethnographic case study is particularly suitable for confined spaces such as offices. Advice Company's employees across all three locations perceive a distinct atmosphere in each of the three offices, even though the city office and the main office are comparable in their physical set-up. Therefore, I will next relate these notions of atmosphere to Advice Company's internal differentiation strategies. As I sat in each of the offices for extended periods of time, I was reminded of Simone Egger's argument that ethnographic research can be regarded as a constant empathetic record of perceived atmospheres, descriptions of localities and experienced sceneries (2015: 159), and that atmospheres require exposure to be received (Rauh 2012: 106). A practical approach of capturing the atmosphere in these three localities was to triangulate the employees' accounts of their office atmosphere with my own percep- 
tions $^{3}$, following Thomas Stodulka's quest to acknowledge the epistemic value of the researcher's affective states and emotions as part of ethnographic reality (2014: 86).

\subsubsection{Main office: Centre of desire or location of distraction and fear?}

The multi-level entry procedures that enclose the main office stand in contrast to the office's obstruction-free open plan office architecture. The interior office space conveys the value of "openness" - which is communicated in the new employee introduction training (see Chapter 4, Section 4.3.2) - to all who pass through the frosted glass door. Suman, a top-level manager, told me he was proud the office had "made the switch to this open-office culture" when the teams moved from their previous location in the city centre:

You know, we got rid of all the office cabins and have everybody now in this open office space. This structure has enabled a lot quicker turnaround time of our projects and of issue resolution. When I need five people for a decision, I get up from my desk, fetch them and we go to a cabin to discuss. After 15 minutes the topic is done, it's so simple. And when I am here, I am always approachable for everyone; people can just walk up to my desk.

Indeed, the open plan office was perceived by the employees to facilitate interaction, but their comments held an ambiguous undertone that can be summarised in the seemingly paradoxical statement of consultant Ruchika: "During a busy day in the office I don't get much done..."

How could a busy atmosphere lead to an employee's perception of not getting "things done"? This question emphasises the fact that work outputs (e.g. presentations and documents) are the sole focus of attention in Advice Company, as most of the annual performance measurement items centre on these deliverables. There is an official assumption about how such outputs are produced, as mirrored in the quote of top-manager Suman, who conveyed an idea of how employees in this office structured their workday "so simply" in order to progress their tasks. Yet what really happens up to the point that the final presentation or documentation is delivered remains in the "black box of

While Rauh (2012) suggests an auto-experiential approach as a philosophical methodology to the analysis of atmospheres, I suggest a triangulated approach that combines interlocutors' statements, observed behaviours and anthropologists' own reports of affectual states. 
organisational functioning" (Czarniawska 1997) and becomes subsumed under the consultant's diffuse notion of "being busy". In order to get a grip on this seemingly subjective notion of atmosphere in the office I took what I call "activity snapshots" of the colleagues I accompanied: I tracked the flow of information over the duration of an hour through different communication channels such as email, chat, phone calls (on mobile phones or landlines), face-to-face conversations and ad-hoc mini meetings of three to five persons at the interlocutor's desk. I use this data on a broader scale in Chapter 6 (Section 6.1) to illustrate differences in job types between the departments.

To convey a notion of the main office's atmosphere, I combined activity snapshot data with a detailed protocol of events during one of Ruchika's "being busy" phases ${ }^{4}$. Ruchika was a client consultant who also led a team of three junior consultants. The following vignette reproduces an hour of one of Ruchika's workdays, during which I accompanied her. Figure 5 illustrates in five-minute intervals - the activities that occurred during this hour. Most occurred in dense sequential order, and some even in parallel.

Figure 5: Communication density in the main office

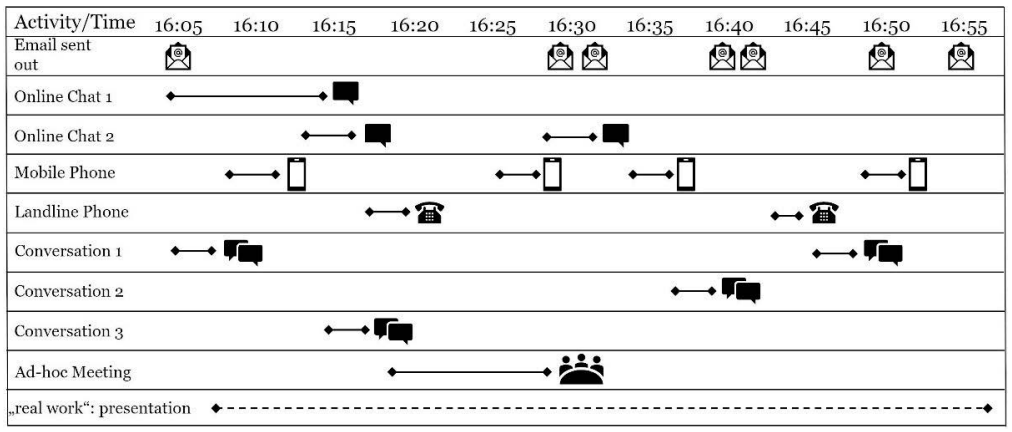

Ruchika had intended to work on a presentation she needed to finish that day. The hour started with her writing a short email to her manager, but this was interrupted when Raveena, one of her mentees, leaned over her desk's

4 Parts of this section have been published in the article "During a busy day I don't get much done" - On the materiality of immaterial labour in a multinational professional services firm (Mörike 2018). 
partition wall to ask Ruchika's advice on replying to a particular client's request. They exchanged a few sentences and Ruchika moved her attention back to her screen. An orange blinking bar appeared at the bottom of her desktop, indicating that she had received an online chat message from a colleague. While she started to respond, her mobile phone rang: a client was calling her to discuss the details of a report Ruchika had sent a few days ago. Ruchika got up from her desk and moved to a corner of the office, where she paced, staring with concentration at the carpeted floor. After a few minutes, Ruchika returned to her desk and opened the chat program to find the name of the colleague she was looking for. She double-clicked his name and sent him a question. While she waited for his reply she finished her still open chat conversation from the beginning of the hour and closed the window. When the reply arrived from the colleague she had reached out to, she wrote back, only to realise that the recipient of her message was already standing at her desk. He pulled a vacant chair from a temporarily unattended desk in the adjacent bay and, together, he and Ruchika leaned over her computer screen and discussed the report documentation. When they did not seem to come to a conclusion, Ruchika stretched out her arm to the landline phone and dialled the four-digit number of a fellow client consultant colleague in a parallel team she knew by heart, without picking up the handset. After two ringtones the call was answered with a "Hallo?" on the other side. A conversation started amongst the three colleagues when it became clear that the issue could not be clarified in a few sentences, the colleague on the phone announced to come over in a second. Indeed, a few seconds later, a man appeared from somewhere behind the meeting rooms and joined Ruchika and the other colleague. Now, all three leaned over the screen, discussing the content. One scribbled a few lines on a note pad and Ruchika moved text boxes around in the document.

Then Ruchika's mobile phone rang again. She checked the incoming caller's name, which was blinking on her phone, rose from her chair and waved with her free hand, signalling for the two colleagues at her desk and her teammates at the surrounding desks to lower their voices. While picking up the phone call she again walked over to the corner and the third colleague, who had initially stood behind the two, took her seat. During her absence, he and the other colleague continued to change the file on Ruchika's laptop. After she returned, she leaned for another few minutes between the chairs over the two colleagues working at her desk, while they came to a conclusion. Both of the colleagues stood up to return to their desks and Ruchika took her seat again. She worked on the jointly discussed document for a few minutes 
while exchanging chat messages with one of the two colleagues about the project. Then she wrote an email to her team about the client call she had just received and another to a colleague in the accounting team.

Shortly later, she again stood up from her desk, walked away and called back the client who had led to the previous discussion. Once she was back at her desk, her manager casually walked by and asked her about the status of the presentation she was supposed to finish by the end of the day. He also asked if she knew the status of a different project in a critical status. She showed him the presentation slides and they briefly discussed them. When she was on her own again, she wrote two short emails before calling one of the two recipients to announce that she had sent him an email with high priority and she would like him to take care of it today because "the client is expecting me to revert back A.S.A.P."

The moment she hung up the phone, her mentee Raveena rose from her chair and leaned over with a question about how she should approach a task she had been given. Raveena picked up her laptop and leaned it over the partition panel to illustrate her issue. Ruchika's explanation was interrupted by the ringtone of her mobile phone. This time she checked the incoming caller's name and answered the call with "Ek second, thik hai? [One second, okay?]". Ruchika lowered the mobile from her ear down to hip level and continued to instruct her mentee for half a minute. Then she attended to the colleague who was waiting on the mobile phone. He was working from home that day and she forwarded him an email while on the phone with him. Towards the end of the tracking hour, Ruchika finally returned to the presentation slides, only to switch back to the email program a few minutes later to write an email she had "almost forgotten". In total, the hour resulted in 14 conversations and 7 written emails, but only a few clicks in the presentation - her "real work", as she explained to me, which she had meant to work on.

When we both sat in the rickshaw that evening (as we were incidentally headed to destinations in the same area that day), Ruchika explained that it had been yet another "of those busy days where you're exhausted in the end and don't really know why, as all the work still remains". She was not aware of the number of communications she had managed in that hour and at a similar scale along the entire day, but she knew she would have to work on the presentation at home that evening.

When the topic was discussed over evening snacks, a colleague who had been with the organisation since before the office had changed locations stated: "This feeling of having done nothing at the end of the day is much 
more since we have moved here." This notion of the office as a "great place to coordinate things and meet people, but not to do the real concentration work" was voiced by colleagues from different functions and hierarchy levels. Sujata from the HR department, with a completely different function and work profile from Ruchika, stated:

To do routine things and coordination I need to be in [the] office, but for creative and concentrated work I want to be at home, have my bed and my things around me, get up and think, continue to work. I need to create that one presentation from scratch for next week; I can't do that in the office. Right now it is quiet, but this is because all of them [she gestures with her arm to the empty desks around her] are in meetings, and our team head is not here. But when he is here you will see that there are people coming to talk to him, they have louder conversations, people on the call...

Her colleague Pallavi got up from her chair and jumped into the conversation: "Oh, yes, you should see it! When my boss is there he always drags me into doing some things I haven't planned for and it all gets turned upside down."

The strategy to simply not be present at the main office for concentrated work tasks was practised in various ways. When I met Aranjit from the main office one early morning at the city office's reception, I asked him if he had a meeting that day at the city office and he replied: „No, I sometimes come here because it is easier to concentrate, when there is urgent work to do. In the main office there are more people approaching me with several work questions, or also non-project related things, that simply distract. Here I can be more focused.“

I met Sneha, a member of the accounting team, regularly when I came to the main office on Saturdays. She remarked that she preferred to come in on the weekend for a few hours to quietly work on tasks requiring concentration over a longer period of time, as she was not able to "properly take care of them in the hectic of the week". Raghunandan, a top-level manager, explained to me that he did his "brainwork" from home in the early morning until 9.00am, and then came to the main office to do "all the chatting with the junior people to give them attention, but also to get a feeling for the potential goof-up of a project before it makes its way through all of the hierarchy".

The main office atmosphere, as perceived by the employees who were based there, was marked by a high communication density and interactions fostered by the open plan set-up. This was the place where "things are happening". But this stood in contrast to notions of "real work" - a working mis- 
understanding that required constant negotiation. But it was not only the colleagues who permanently worked at the main office who reflected on its atmosphere. Similarly, employees from other offices who occasionally came to the main office contrasted its atmosphere to that of their own office. Payul, who was based at the street office but had to work at the main office every few weeks, remarked: „It is usually very loud and bustling at our place. This is why it is for me always a bit weird to work at the main office, where all is so like muted and I have to speak in such a low voice there."

She also recalled several situations in which colleagues with whom she was collaborating at the main office indicated that she should lower her voice. On my first day at the city office, Ananya asked me about my research. When I told her that I had spent several months at the main office before coming to the city office she said that I must have noticed that the main office was "a much more corporate place" than her office. Noting my questioning expression, she explained:

A: Here it is not very corporate, people are not in formals $s^{5}$ and we also don't have many meetings. Most of my work is sitting here and talking to the team around me. But as people get more advanced in their career they also have more meetings and this is how the main office is much more corporate.

FM: In how far is this corporate?

A: Well, the senior managers are all meeting much more people. I also meet people, but they are only my colleagues. But at the main office they must all be having client meetings much more. It is the place where all the managers are and clients come.

Sneha and Payul characterised the main office's atmosphere as a consequence of its employees' advancement along the career ladder, which resulted in seriousness and a more silent code of conduct. Other colleagues, however, sensed an atmosphere of fear at the main office. One morning in the main office I was on my way to the water cooler when I met Devan, a team lead from the city office, where I had accompanied colleagues from his team. Before I could

i.e. a formal dress code, consisting of a business suit or at least trousers and a longsleeved shirt for males and a business costume or suit for females. Alternatively, a three-piece Indian dress, such as a salwaar kameez or a kurti combination, is sometimes worn. 
formulate a question about his perception of the main office, he addressed the topic by himself:

If you ask me about the differences between the two offices, I'd say that over at the city office is more an atmosphere of fun factor [...] Here [at the main office] it is more an atmosphere of fear. Fear is necessary for success, right? So people pick up the phone a few times per day and shout into the phone that they want this and that right now for their clients. No wonder that when somebody hears that from Monday to Wednesday, he will pick up the phone on Thursday just in the very same way. Given a choice I definitely prefer this office here [main office], because... well, it is nice to have a chilled fun atmosphere for some time, but people here are great, they move forward.

On the one hand, this quote illustrates the perceived connection between career achievement and fear in the main office's atmosphere. On the other hand, Devan confirms, through his example of the aggressive phone communication style, the assumption of Systems Theory that individuals within a social system behave according to the framework set by the system: independent of individual traits, team members adopt a group's behavioural patterns, which can be processed in their respective systems. Devan's observation corresponds with Ruchika's self-observation of the way in which her own behaviour varied between the two consulting teams (see Chapter 6, Section 6.1.1). Sameer from the city office also perceived a tension at the main office, but connected this to accountability: „In the main office people are more working for themselves, they own the project; it is theirs - and their head on the table. If they do well, it is their remit, if they mess up, it is their problem. And that's the tension there."

When connecting this perceived "atmosphere of fear" to the intense physical vocabulary used by Gopal - a client consultant who was managing a project that was hanging in the balance - this atmosphere seems tangible. When Gopal described his problematic project, he claimed that he would be "dead meat" if he did not manage it correctly. Then he commented on a crisis meeting that had occurred the day before: "There was a lot of heat yesterday night and we have talked to the other team, but not in a very corporate way. We had to bash them up."

Here, Gopal repeats the idea of a "corporate" office in which interactions occur in a moderate manner. In contrast to moderate handling of events stand moments of crisis, when the success of a client project is at risk and when fear of failure causes friction between teams, resulting in perceptions of a 
heated atmosphere. In these instances, behaviour can readily shift towards more aggressive modes of communication, which Gopal compares to physical violence. Sameer attributed the "tension" in the main office's atmosphere to accountability, and this idea corresponds to a situation in which Gopal had to commit to a final project delivery with a client. He stated, while writing the email: "So now will be the moment when I will have to report concrete figures and stick my head out, risking if they are wrong for my head to be chopped off."

The examples illustrate how the main office, as a tempered space, had a distinctive atmosphere that was perceived by all employees, whether or not they were permanent employees at the location. That aspects of the atmosphere might have been actively created brings to mind the words of Raghunandan, a top manager from the main office. Raghunandan stopped me casually one morning at the canteen after I had started to accompany members of a different client consulting team in his department than I had in the weeks prior. He asked me if I could already determine the differences between the teams. Cautiously, I answered that I thought it was too early for me to have insight into the team members' different perceptions, and returned the question to him, asking if he saw any differences. His assessment indicates how the organisation connected fear and success to client centricity:

They [he gestures towards the desks of the team] are not working towards the client's needs. Sometimes the client requires us to work overnight, sometimes several nights, maybe even for a week. But they [the team members] are not willing to do that, so the client does not want to work with us anymore. They are happy, they do not have that much overtime as the others. But Advice Company does not necessarily want only people who are happy - we want more people who are ambitious like they are in the other team. This is why one person left that team, He/she wanted to achieve more. But soon will be a time when I have to... hehe [laughs drily]... increase the pressure there a bit more [he makes a move with his hand as if to increase the volume on a radio].

The short conversation was cut off by the ringtone of his mobile phone. Raghunandan gave me a brief nod and walked away as he spoke into the device. My gaze followed him along the aisle, while I swallowed hard and made a mental note to be even more considerate of what I said during the fieldwork. The gesture with which he accompanied his final words repeated the employees' 
notion of tension, pressure and fear. But he did not perceive this atmosphere; rather, he intended to create it.

The main office was the most client-centric of the three offices, even on the basis of employees' perception of its atmosphere. The office's cumulative texture as a "corporate place" that each employee characterised differently having an air of seriousness due to employees' achievements and career advancement, defined by contact with clients (i.e. the environment) or showing a high code of conduct through moderate talking (no loud speaking, no "bashing up"), a different dress code ("all formals") and ambitiousness ("people here want to move forward") - marked its atmosphere as distinct, relative to the other offices. The idea of atmosphere is appropriate for an analysis of the organisation's internal differentiation, as all of these notions of the main office's atmosphere correspond to the value client centricity as the leading selection criteria.

The analytical focus on atmosphere as a tempered space allows, however, for more differentiated and contradicting views of Advice Company's main office. The notion of the "corporate" office does not seem to fit the highly interactive work atmosphere, which employees perceived as distracting. Employees even sought to avoid this atmosphere by moving "brainwork" to less "corporate" and client-centric spaces - in both a territorial (home vs. city office) and a temporal (early vs. late hours vs. weekend) sense. Employees' articulations centred on the completion of work tasks, especially with regards to accountability to clients - the environmental system that Advice Company required to sustain itself and the key selection criteria for internal success, prestige and status. This accountability built an atmosphere of fear, which was physically perceptible not only to the employees who permanently worked at the main office, but also by those who visited the tempered space with its wellequipped, open plan design guarded by multi-level entry procedures.

That employees perceived the main office to be located at the top of the organisation's value system powerfully illustrates the idea that, on the one hand, closeness to clients was connected to accountability, prestige and status, which found its bodily correspondence in formal dress and voice control; but on the other hand, this proximity to clients was also connected with fear, tension and pressure, as manifested in the physical characteristics of exhaustive communication density, aggressive shouting and metaphors of execution. Thus, the atmosphere of the main office could be characterised by a predicament: rising high to the bright centre of client centricity came with a significant risk of getting burned. 


\subsubsection{City office: Fun zone with $100 \%$ accuracy commitment}

The employees at the city office support the client consulting teams with projects, often by preparing presentation files. But they not only support their colleagues at the main office; in addition, the majority of the teams at the city office support client consulting colleagues across the globe. In a classic scenario of offshoring and business process outsourcing (Upadhya 2016: 44-45), the teams at the city office work for colleagues in the US, Europe, Australia and Asia. I was told that three to four years ago, positions in this office had been offered to "freshers" - college graduates in their early 20 s who were exploring their first job after earning their bachelor's degree. Now, Advice Company only employs graduates with at least a master's degree. As a consequence, the newer cohort of support analysts are three to five years older than the previous cohort was, and several have work experience from other organisations. In this respect, the newer employees in the city office are not substantially different from those starting entry-level jobs at the main office, apart from the fact that their degrees have generally been issued by lower-ranking universities. This has a direct bearing on the office's position on the client centricity scale, as Sujata from the HR department stated:

We do campus recruitment for this [city] office here at similar B[usiness]schools then to the consultant jobs [at the main office], but with slightly different ranking profile. What they do here is actually a similar work, but it is regarded less qualified because it does not involve direct client interaction. It should not be that way, but in general this is what happens.

While I had first entered the main office with no prior information about it, I was led to have big expectations of the city office. Whenever I mentioned my upcoming research phase there, reactions were remarkably similar to Raveena's: „Oh, you'll be at the city office for the next weeks?? Awesome, you will have soooo much fun there, it's such a young crowd, a real young people's place and a really good atmosphere." With great excitement I looked forward to this research phase in the city office. From the reactions and descriptions I had received, I imagined the atmosphere there to be an appealing blend of a perpetual school trip and a graduation party. The quote from Devan in the previous section compared the main office to the city office, and he reiterated the image of the city office as a young person's place with a "chilled and fun atmosphere". This fit the quotes and remarks from many other employees at both locations. The perceived atmosphere of the city office varied significantly 
to that of the main office, despite a similar yet downsized office set-up, less equipment and less sophisticated entry procedures.

At the beginning of the fieldwork phase in the city office I was introduced to Sanjay, the office manager. He pointed out that his expectation of his employees was "100\% accuracy in the work they deliver" and that there was a "close to zero tolerance on mistakes". He conveyed this requirement as "a very, very clear message" to new joiners. Indeed, the newly joined colleagues I accompanied or talked to, such as Imran, mentioned this strict, zero tolerance rule, which led him to act with high caution. Imran had developed a routine of double-checking his work before sending it to the colleagues he supported (see Chapter 10, Section 10.6). This cautiousness was also reflected in the requirement that all presentations had to be reviewed at least twice by peers for errors in formatting or numerical mistakes before they could be sent to colleagues abroad or in the main office. Accordingly, quiet rumours about colleagues who had repeatedly produced errors in their work and were asked to leave circulated throughout the office. The 100\% accuracy work target as a condition for organisational membership and the everyday work practice of reviews did not seem to fit with the "chilled fun place" atmosphere other employees had reported. Similarly, the data I collected through my hourly communication snapshots and observations of working and collaboration practices did not reveal major differences to the main office. Nonetheless, I realised that I had gradually changed my clothing to jeans and a T-shirt, which I had rarely worn at the main office. In the city office, I came to work in the local kurti/patiala combination only when some female office colleagues agreed that we would wear "formals" the next day to "click some pictures together". So the atmosphere was indeed different in this office.

As my research continued, my conclusion crystallised that the perceived atmosphere at the city office could be understood as the result of work practices that were intersected by memorative narratives of the place in times past. Specifically, this past referred to three to four years prior, which younger colleagues considered the "fun zone". Monique had worked for Advice Company for five years and had recently returned to the city office after working in the main office. She had come back because she liked it better in the city office, even though - in the main office - she had had exposure to other teams and direct interaction with clients. When I mentioned that I was told I would find this office a "fun place", she replied: „Well, it used to be that way, but now people are also more serious, I have noticed since I am back here. Before I left, people would randomly pick up a guitar and play some chords when they 
felt like taking a break. Now the recruits are MBAs and postgrads, so they are a bit more serious, but still good. I really like it."

Attul had joined the department more than three years ago as a fresher and was, in his own words, "one of the few college passouts [with bachelor's degrees] remaining here". Similarly to Monique, he had noted a change over the past few years, which he also attributed to the different entry conditions:

As they [Advice Company] increased the package [the salary], there are now only MBA students here with a different way, different culture. These guys have to work to pay off the debts from their grad schools; we college people were less forced to earn and always had an option to do something different. I have seen this place developing. When I came, it was still a type of trial and error. But it was a great atmosphere here. We used to have so much fun, people were sitting on each other's desks, chit-chatting, going out together for a smoke. But we also did work, there were times when I worked from 10.00am until midnight!

These recollections of the "great and fun atmosphere" related to the past - the early days of the department - and were repeated and retold within the office. In contrast, colleagues working at the main office had a different perspective of the city office. These colleagues connected the city office with their memories of the location as the organisational headquarters at an earlier point in their career, when they presumably had less responsibility, accountability and pressure. Only a handful of my interlocutors from the main office with whom I talked about the city office had actually been there since the headquarters had moved, and even fewer could back up their knowledge about the city office with insight into the current situation. Apart from the HR colleagues, no one was aware that only master's graduates were now being employed in the "young people's fun place".

When recalling their former office, the main office employees highlighted a number of advantages: they perceived it as more cosy and a place in which people used to have lunch together, because the lights would be switched off between 1.00 and $2.00 \mathrm{pm}$ and every employee would bring their lunch and eat during that time. In addition, for after-work gatherings, the city office location had much more to offer. The recollections and reverberations of this "fun atmosphere" by the main office employees meant that they still perceived this as the de facto situation. 


\subsubsection{Street office: The vivid interaction hub}

Similar to my response from colleagues about the city office, comments were almost guaranteed when I mentioned my upcoming research phase or a planned visit to the street office. I would receive reactions such as this one from Preeti, a project coordinator: "What do you want there, it is loud and they don't even have a proper washroom." Others gave me an elaborate description of the upstairs office space, with its narrow desks and plastic chairs, without mentioning that this was the freelancers' exchange zone. All this prepared me to expect a campsite rather than an actual office.

Hence, I was surprised to find an air conditioned workspace, albeit one with a low ceiling. While the environmental characteristics of the workspace certainly had an impact, the perception of a specific atmosphere only unfolded after I was exposed to the setting over a longer period of time. The restricted space necessitated narrow, rocky office chairs, which abutted each other on opposite sides of the desk bays. Even with the greatest care, the chairs' backs would touch each other whenever one of the two colleagues would get up or look for documents from the piles that mounted up beneath each desk. Whenever there was a teleconference, landline phones would be set to speaker mode and the characteristic metallically blurred voices would sound through the room. During the afternoon, two or three conference calls would often happen in parallel and voices would blare from several corners of the office. The computer space next to Mudra, who I accompanied on my first day, was shared between several employees. Over the course of the day, four people would use it for a good hour before leaving again. The manager's desks, which were clearly marked by movable dividers, would at any given moment be used by at least one of their team members, who would either pull a chair to a corner of the desk (where they would place their laptops) or sit directly at the desk, leaning over their supervisor's screen.

Although this office area was for the Advice Company employees and not the dedicated zone for the freelancers, the environmental characteristics here differed substantially to that of the other two offices. This difference extended beyond the differences in equipment: loud voices from telephone speakers dominated the acoustic setting and employees only sat at their desks during conference calls or when they had to urgently complete a task on the computer. Otherwise, they stood together in small groups, whose composition changed frequently. 
The atmosphere in the office was marked by the constant "rushing in and out" (Mudra) of colleagues during the day - as required by their role. They would come into the office only to check their emails or attend a teleconference, or to talk with the freelancer teams upstairs. Upon returning to the office space, they would transform their updates and feedback into emails and other communication formats that could be processed within the system of Advice Company. For my unaccustomed brain, the average sound intensity and the constant flow of changing communication groups posed a challenge. During the first few days, I got a severe headache after only a few hours, which "improved" gradually to the point that I only became tired and struggled to follow conversations in the afternoon. When I realised that, for Payul, this atmosphere constituted the norm, it became apparent to me how intensely she must have felt the difference in atmosphere to that of the main office.

The atmosphere at the street office mirrored its major function as a hub for managing interactions with the environment, and it was geared up to support this. It allowed for smooth switches between the communication style used in the organisation and a style more suited for interacting with the environment, the freelancers. The "boundary work" with the freelancers had its own requirements, and the employees at the street office evaluated the space primarily from the perspective of its suitability for this function. Payul opined that, in this environment, it was very easy for the freelancers to come in and catch up. She could not imagine them doing this if they had to wear a badge and wait in line at the gate every time: "Here is such a big in and out of people in the course of the day that registration etc. would lead to an issue." When I asked Rohan, who was a main contact person for the freelance teams, if he would want to work at the main office, he instantly replied: "Noooo! This would not work, it is too far out for the freelancers. They would have to spend at least 20 rupees per day on travelling. That is too much."

The clients and client consulting teams were so far away that the employees at the street office characterised themselves as "simple people". This was expressed in opposition to employees at the main office, to which only a few colleagues regularly travelled to attend meetings or to work. Despite the expression of a certain sense of belonging to the street office, the colleagues in this location were well aware of the client-centric orientation of the organisation and their position at the lower end of the value system. Rohan's reflection on his department's position within the organisation connected it to the office location: 
Rohan: [...] But you can see we are not taken serious.

FM: How?

Rohan: ]ust take a look around! Are we a priority? [He gestures to a cramped desk drowning in piles of paper] We were promised to move since many months now.

\subsection{Concluding remarks: Client centricity as a continuum}

This chapter began with an analysis of the organisation's internal differentiation along the value client centricity by comparing the access procedures across the three offices. A subsequent comparison of the internal set-up and equipment of each office illustrated an internal differentiation of the three offices on a continuum based on distance to the client. The employees' notions of office atmosphere corresponded to the set-up, but provided a layer of contradicting views.

Based on this analysis, I have shown how the three offices emerge as subsystems within the organisation along the central value client centricity (Figure 6). But instead of providing a mere dichotomist distinction I have argued that the offices are located on a continuum of client-centric differentiation. The main office clearly occupies the highest position on the client centricity scale, as the office with the closest contact with clients. The street office represents the opposite extreme with respect to both physical space and atmosphere, as well as to structure, as this location manages contact with the freelancer teams. The city office breaks the dichotomy, falling between the other locations on the continuum. Louis Dumont refers to this as hierarchical opposition which rests on the assumption that entities within a whole (i.e. a social system) are arranged in a hierarchical relationship. Dumont holds that hierarchical arrangements occur more or less autonomously. He recognises the hierarchical classification not as isolated, but related to the overall system in which entities are arranged: The fact that subsystems (i.e. offices, departments, teams) belong to a whole is simultaneously the cause and the consequence of their placement in a hierarchy. This "assumes that values in relations are never balanced or equivalent [...] but hierarchical when conceived through and defined in relation to the whole" (Kapferer 2011). 
Figure 6: Client centricity scale

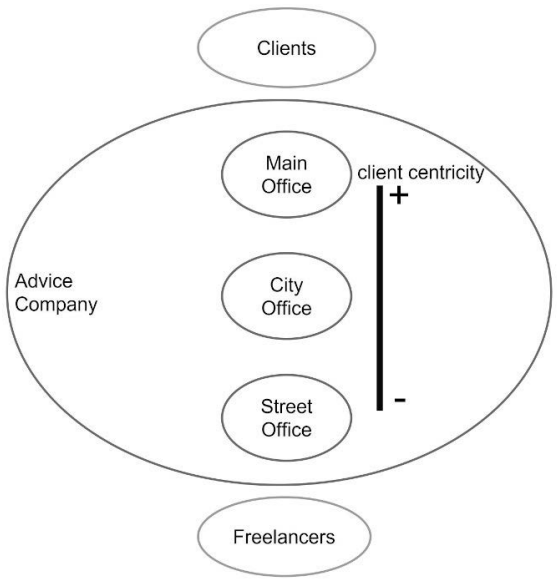

The city centre location occupies the middle ground between the two ends of the continuum for several reasons. First, it previously hosted the most client-centric functions of the organisation and the relics of that past are still visible. A two-stage access procedure is in place, yet not as stringent as that of the main office. The equipment is comparable to that of the main office, but the set-up mirrors traditional organisational structures with desk space representing the organisational hierarchy. Second, the employees, themselves, are in a middle position on the client centricity scale. They have largely entered the organisation from a third-level university, and some only have an undergraduate degree. For this reason, they do not sit at the main office amongst the teams that service and consult clients. They feel "behind the wall" (Section 10.5), supporting global colleagues on client projects in a downstream position, where "client centricity" means 100\% accuracy. But they are in striking distance of achieving a more client-centric function. In contrast to the street office employees, the employees in this office could potentially move from their position "behind the wall" into the client consulting teams, either in the main office or in one of Advice Company's many other offices across the world.

However, the analysis of the atmosphere across the three offices, as perceived by the employees, has shown that, in addition to the distinctions be- 
tween offices on the basis of client centricity, there are also contradictions in the one-directional alignment of the organisation to the client. At the main office, notions of fear, together with the strategy of employees to avoid the atmosphere of high communication density and to travel to less client-centric locations (such as the city office) to perform "real work", indicate an antithetic value according to which selections are made. This value is also reflected in the words of Monique, from the city office, who returned to the office after a stint at the main office. The street office, with its loud and not "corporate" atmosphere, was not only seen to directly oppose client centricity, but was also perceived to be structured according to a different value, in which interaction with freelancers was most important and information was selected accordingly. When client projects go wrong at the main office and pressure rises, the communication style becomes less "corporate" - similar to that of the street office. This indicates that client centricity is apparently counterbalanced by an opposing value. This opposing guiding difference is orientated by a different system in the Advice Company environment - the freelancers - and the employees referred to it as the "ground reality". The street office was deemed closest to this ground reality, and the main office furthest from it (Figure 7).

Figure 7: Guiding difference client centricity/ground reality

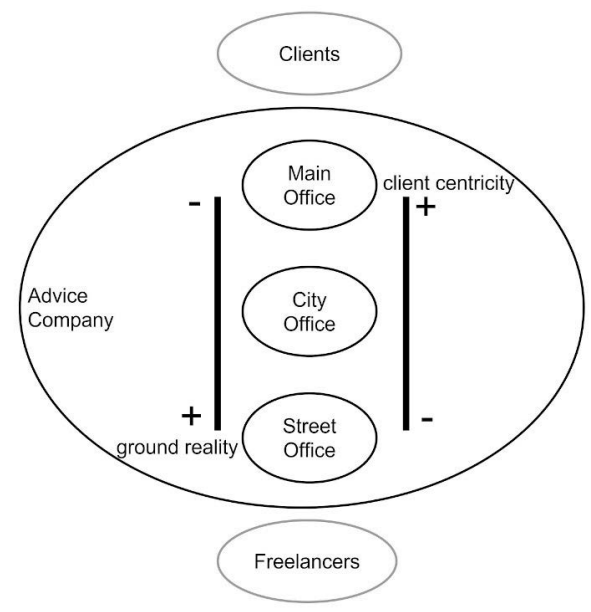


The organisation's guiding difference consists hence of the two opposing and mutually exclusive terms client centricity/ground reality. This relates to Dumont's second proposition that the established hierarchical set-up is neither stationary nor static. Even more, the hierarchical arrangement can be inversed for a limited time and in specific situations:

The reversal is built-in: the moment the second function is defined, it entails the reversal for the situations belonging to it. That is to say, hierarchy is bidimensional, it bears not only on the entities considered but also on the corresponding situations, and this bidimensionality entails the reversal. (Dumont, 1980 [1966]: 225) 



\section{Formal Boundaries, Informal Bridges: Departments and Teams}

Drawing on Advice Company's internal differentiation into the three offices as sub-systems, this chapter continues with an analysis of further organisational differentiation within each office. With a focus on functional and hierarchical differentiation, I will elaborate in Section 6.1 on the boundaries between the sub-systems across the open office floor. Starting with the functional divisions that stem from the organisational chart, I will trace how different teams emerge as sub-systems on the basis of their communication patterns and the self-observations of interlocutors. By presenting case studies of such sub-system in each of the three offices, I will illustrate that these perceived boundaries are consistent across the organisation and reinforce the meta-structure of the organisational system that is aligned with the dominant value client centricity. While this proposition holds for a number of cases, the internal differentiation at the street office implies that the client centricity scale is here reversed in a sense of Louis Dumont's hierarchical inversion, as it instead orientates along the value ground reality.

Section 6.2 focuses on further system differentiation and illustrates the emergence of sub-systems that do not necessarily adhere to the hierarchical or functional structure. Luhmann refers to such sub-systems as "conform/deviant" (1995a: 190). I will analyse these sub-systems in light of the structure developed in Section 6.1, as these deviant sub-systems constitute an egalitarian structure that is not connected to the formal structure of the organisation.

\subsection{Differentiating function and hierarchy: Job types and teams}

While the previous chapter demonstrated that the three offices can be placed on a continuum of client centricity, with distance to the client as the key crite- 
rion for positioning, this section will illustrate how the differentiation of the organisation continues beyond the emergence of sub-systems at each of the three offices. Starting with a basic description of the functional differentiation of departments and teams in the main office and the corresponding work patterns observed there, I will show how individual teams emerge as sub-systems that strongly emphasise their boundaries with other teams, despite a lack of physical divides in the office floor. Along with the functional differentiation, a differentiation of sub-systems into hierarchically organised clusters, which emerge along the superior value client centricity, becomes salient. In the city and street offices, an emergence of both functional and hierarchical sub-systems can be shown.

\subsubsection{Main office: Working around client centricity}

As described in Chapter 5 (Section 5.3.1), the main office is an open plan space with 450 desks of identical size and equipment, and no physical divides (such as walls or offices) to mark departments. However, the office yields a clear territorial divide that becomes salient when one looks at the basic functional differentiation of departments and teams. The teams are, on the one hand, assigned to job roles such as client consulting, project coordination and HR management; but on the other hand, they are also assigned to particular clients and industry sectors. However, not all job roles are affiliated with a client or industry team: The accounting experts, for example, support all projects by creating contracts and invoices, while HR and finance colleagues have no connection at all to client projects. The diversity of the HR and finance team was emphasised in various ways during my meetings, by members of both these and other teams. A senior management board member opened his talk in the main office's central meeting room (to which all employees were invited) with the following statement:

Do we have finance or HR people in here today? Raise your hands... er-hm, quite a few. Well, you might not understand all of what I am saying, but l'd appreciate if you guys look interested.

HR team member Sujata introduced herself at a meeting by making a similar reference to the distinctiveness of her role from that of the other speakers that day: 
Hi all together I am Sujata, for those who haven't met me before. I am from the HR team here, so in this session it is not about business and markets, which I know just little about, but about collaboration.

These departments' positions as sub-systems set apart from the organisation's client-centric structure, yet with a substantial degree of relevance, are mirrored by their location in a corner of the office next to the desks of the top managers from all departments, including the CEO. The entire HR/finance/management area is "shielded" by several desk rows of teams that lack a client or industry affiliation, such as the data organisation and accounting teams. The remaining desks in the main office are assigned to client or industry teams and represent the hot spots of everyday action. The involvement into everyday work with or for clients is the is the first dimension of differentiation along which the sub-systems establish their internal boundaries. The client and industry teams have two further criteria along which sub-systems emerge: job type (client consultant or project coordinator) and client/industry type. The organisational set-up assumes close collaboration between the job types needed to execute the entire project development process. For example, a client consultant must collaborate closely with a project coordinator to execute a client project. Hence, I initially expected sub-systems to emerge in correspondence with client teams.

The actual differentiation, however, arises in the first instance according to job types. In meetings involving employees of several job types from different client teams, consultants communicate as a social system in opposition to project coordinators, and individuals are described by other teams and roles as "this client consultant lady" or "this project coordination guy", rather than "the consultant from the client ABC team". Project coordinator Asif expressed this notion of being identified primarily by his function when he commented on the accountability he felt as a project coordinator: "It is me who is working on the project, but it is the project coordinators who did it all wrong, who screwed up."

The differentiation between job types mirrors the continuum of client centricity and hence imposes a hierarchy, with the client consultants - those with the most direct interaction with clients - in the highest position. Project coordinator Nimesh expressed this explicitly, although he chose a surrogate to make his point: "I have seen my colleagues in the team being treated like a personal helper. We have to be equal; none of us should feel inferior to client consultants." 
The fact that client centricity is the primary factor assigning system membership shows that this value is the leading one in this context, and not ground reality. These boundaries become salient from another angle, when looking at the communication patterns of the various job types. Data collected in onehour "snapshots" of the observable communication of interlocutors across various channels enabled me to develop distinct communication profiles. I analysed a set of comparable snapshot data that had been collected during hours in which the employees perceived their workload as medium-high to high and there were no scheduled meetings/trainings. After plotting the incidents of incoming/outgoing communication per communication channel on a multi-dimensional radar chart, a pattern emerged across five to six employees of the same job type. For client consultants, a strong emphasis on personal (one-to-one) communication was noticeable (Figure 8), while project coordinators conducted a significant amount of their communication via the landline phone (Figure 9). This data illustrates that the job types are not only functionally different, but their work and communication styles also significantly diverge.

Figure 8 (left): Communication patterns of client consultants

Figure 9 (right): Communication patterns of project coordinators

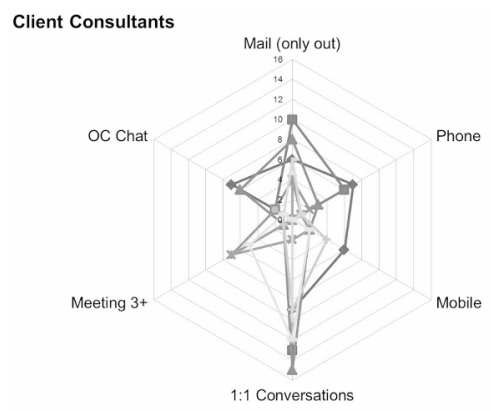

Project Coordinators

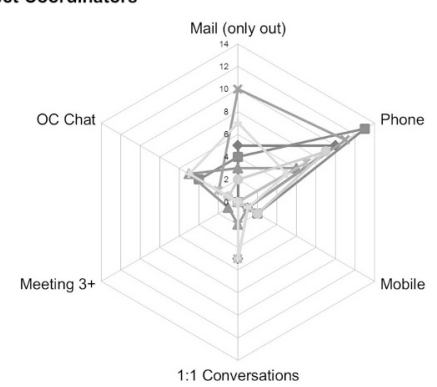

Given the assumption of the organisational set-up that each consultant will collaborate with the project coordinator for a client project, the question arises via which communication channel this collaboration can be realised. The snapshot data suggests a further explanation for why the client teams do not evolve as social systems across different job types: they have only a narrow set of communication channels in common; hence, they have fewer opportu- 
nities to establish a social sub-system through communication. I do not wish to indicate that the two job types do not communicate with each other at all. Advice Company is dependent on their interaction for the maintenance of the organisation. But the data supports the employees' perceptions, which I will present in the next pages, that they operate in separate sub-systems. This is based on the assumption of Systems Theory that without communication, no social system can emerge.

Figure 8 shows the client consultants' strong emphasis on direct face-toface interaction. My observations suggest that this communications predominantly occur within their team: several work tasks are performed by two or three members of the same consulting team (who sit together at one desk) closely interacting and therefore establishing and reconfirming their team as a social system distinct from other systems in the organisation. Therefore the sub-systems emerge according to the specific client teams. This communication pattern also fits with the territorial closeness of the consulting team members, whose desks are located in the same bay.

Consequently, consultants of one client team perceive their team as distinct to other client consulting teams, because the communication events within their team lead to differentiation and boundaries with other teams are explicitly communicated. When I asked Raj, a consultant, to elaborate on his statement that one client consulting team was more problematic than the others, he said that client team A was very stressed out because their customer was extremely demanding. Client consultant Kashish once commented about his team: "The time in my team is for one month as if it was a year in the rest of Advice Company." Ruchika, who had switched from one consulting team to another, commented on her previous team:

Working in that team under that manager brought out the worst in me. I did not know I could behave like that, that I could write emails like that to my colleagues. But with that team it was simply the only way for me, and here in my current team it is very different now.

Her quote also underlines that members of a social system act according to the system's framework, and not on the basis of individual traits or preferences. The constitutive strength of client centricity as a central value in the organisation is illustrated by observations that the emergence of a sub-system such as the client consulting team can only be fostered through physical proximity once the overarching boundary between job types - along the value client centricity - is established. 
This stands in line with Luhmann's Systems Theory, according to which sub-systems emerge by repeating the established boundaries of the superordinate system - in this case, the job type divide. The communication between a consultant and a project coordinator who sits right across the desk row is conducted over the phone, chat or email, but rarely via one-to-one conversation. Anjali, a project coordinator, said that she found it problematic that she was assigned a desk close to the consulting team, whose projects she mainly managed, rather than other project coordinators:

When I asked [why I had to sit here] I was told that it is due to the interaction with the consulting people, but that is not really true. Copal [a consultant sitting two desks next to her] for example also just calls me via phone or sends a chat instead of getting up and going to my desk. So what is that argument!?

She also said that she missed team functions, as she was not part of the consulting teams and the coordinators did not host any functions. Hence, the physical proximity of project coordinators to their consulting colleagues did not overcome the boundaries between the job types, but prevented the emergence of project coordinators as a distinctive sub-system within the organisation. The project coordinator's relatively low share of one-to-one conversations as a strong system-constitutive communication channel corresponded with their dispersal across the office area.

\subsubsection{City office: Same but different}

As described in Chapter 4, the city office has a similar set-up to that of the main office. It has an open plan office and no walls except for a shielded row of cubicles for team managers and a separate office for the department manager. In contrast to the main office, however, the city office shows a significantly more homogeneous functional subdivision of teams: all teams have the purpose of supporting colleagues across the globe, and most teams on the ground floor fulfil this function by creating presentation files based on the data they receive and the formatting guidelines they are given. Yet, right at the beginning one of the team managers described the organisational structure and pointed out a distinct difference in job types across the departments. He felt that the difference between "embedded" teams and "standard" teams was important for my analysis: Employees of embedded teams are assigned to a client consulting team abroad which they mostly support exclusively. In 
contrast, standard teams act as a "pool of resources" to which work requests from several client consulting teams are submitted. The local standard team manager at the city office distributes requests amongst the team members on the basis of each individual's workload. But the requests not only come from colleagues abroad, but also from embedded colleagues. Both embedded and standard teams have a local team lead and a manager in the city office. But employees in the embedded teams receive their primary feedback from their overseas managers and view their local team leads as contact persons rather than a line managers.

The decision of whether a person will join an embedded or a standard team is made at the hiring stage: Advice Company offers either the former or the latter job type at the campus recruitment events, on the basis of university's ranking. Inside the city office, a member of a standard team can switch to an embedded team after one or two years, via a promotion for good performance. This notion of a hierarchical differentiation of the two job types was reflected in informal conversations between colleagues. When I accompanied Niharika from the standard team, Anas (embedded team) walked by and made a joke about the embedded analysts versus the standard ones, and that Niharika should be honoured that he had come to talk to her. They teased each other a bit more and Niharika turned to me with a laugh: "You know, people in the embedded teams don't talk with the standard teams, usually they don't interact much." Although this conversation happened in a casual, friendly context, the boundary between the two sub-systems was clearly communicated.

As explained above, the hierarchical differentiation is dependent on the universities from which employees have graduated, as an embedded team member commented during a casual coffee chat in the cafeteria: "The difference between embedded and standards? You really haven't noticed? I shouldn't say that, but look from which colleges they [the standards] come from." The differentiation is also connected to the client centricity continuum. Tauseef, an embedded team member, explained his job to me by presenting it in opposition to the standard team job:

We are owning the project, we take responsibility. See, that is the difference to the standard team, you might have noticed. They don't own the project. They just deliver the task and that's it. But we are more on the client consultant side, the project is our baby, we are fully committed and we are much more committed to the client. See, this work task I am dealing with now we 
usually would have outsourced to the standard team as there is nothing to learn. But unfortunately the standard team is working full capacity and have declined this request.

Entry into the organisation is therefore associated with a hierarchical distinction based on differentiation within the educational system, and this affects employees' perceptions within the organisation. This represents an interesting interplay not only between the organisation and the educational system, but also between the clients as a third system, through the connection of university ranking and proximity to clients.

Similar to the main office, in the city office there seems to be a distinction between job types, with a hierarchical order associated (embedded jobs are more desirable than standard jobs). Within each of the job types, subsystems further develop along regional and client teams. These teams form the sub-systems in which employees primarily perceive themselves to interact when asked about their work-related networks in the office. Similar to the main office employees, the employees here distinguish and characterise their teams from other teams and communicate the team boundaries explicitly. I asked Niharika to explain her comment that one must be careful when taking over work from other teams in order to help them out. She elucidated that, although all were members of the larger standard team, there were differences from team to team. She would look at a project from her team's perspective, but the other team might view a job from a different perspective. This difference in perspective might affect little things such as formatting decisions in the presentation slides, but also larger issues, such as analytical style. Her explanation exposes the sub-systems and the different selection procedures within them.

Ananya, from an embedded team, told me about a project the department manager was using to try to change the entire knowledge management culture. Upon my probing into which kind of culture he was referring to, she explained:

People here are very closed and do not talk to each other much. The team B, for example, does a lot of special analysis, but nobody knows about it. Within my team I know quite well who is doing what, but outside I don't. I would rather ask my manager in Shanghai before I go to a person here I do not talk much with. 
The perception of teams as sub-systems with clear boundaries hence aligned with the formal structure of the organisation. To illustrate these perceptions, I mapped the sub-systems in a seating map of the city office. Figure 10 shows a map of a part of the seating area, with individual teams indicated by different patterns.

Figure 10: City office team map

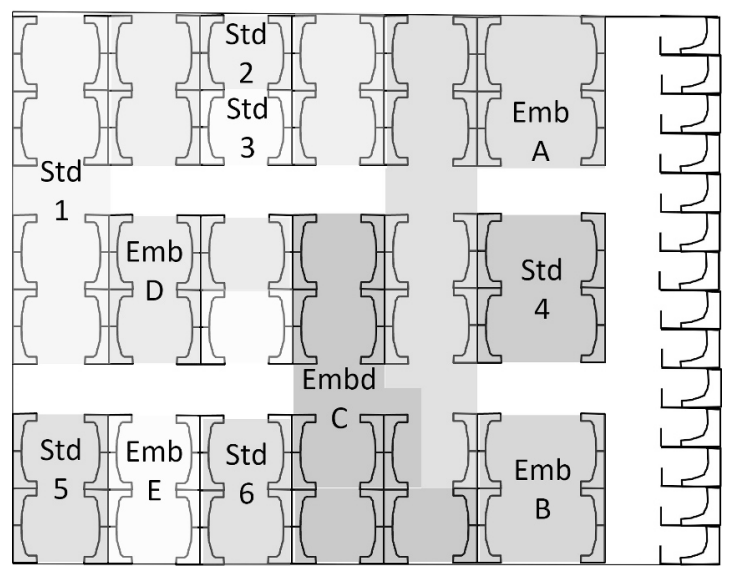

Similar to the differentiation within the main office, the differentiation in the city office shows a primary distinction and assignment of sub-system membership along the guiding difference client centricity/ground reality with client centricity as superior value. This boundary is communicated between the embedded jobs, which are described as more client-centric, and the hierarchically lower standard jobs, which are considered less client-centric. The secondary distinction occurs in line with the organisational matrix, along the distinction of client/regional teams, and is communicated accordingly.

\subsubsection{Street office: Differentiating ground reality}

The work area of the street office consists of two rooms that host up to $50 \mathrm{em}$ ployees. The restricted space around each desk - resulting in physical closeness, shared phones and an interactive working practice between employees, as described in Chapter 5 (Section 5.4.3) - led me to assume relative system homogeneity. I did not expect to find emergent sub-systems across the small 
office space, especially considering that all of the job types performed at the location orientated on the value ground reality, in distinct opposition to client centricity.

My interlocutors, however, differentiated job types at the street office along a boundary between ground reality and client centricity. Consequently, two different sub-systems emerged in this setting. The most prevalent functions at the street office are managing interactions with freelancers and engaging in "boundary work" with Advice Company's environment. A different job type concerns interaction with the main office - specifically aggregating financial figures and managing staff workload. While the latter roles primarily interact with project coordinators at the main office to assign team leads to projects and to communicate this, the former are responsible for the concrete realisation of projects at the street office, including managing the freelancer team leads with respect to the types of projects and work expected from them. Respectively, these employees collect information about the status and progress of work, which is aggregated through the chain of job types (see Chapter 10, Section 10.4).

Similar to the differing communication patterns between consultants and project coordinators at the main office, the communication patterns between the two job types in the street office also diverge. Those whose role is to communicate primarily with the main office align their communication channels to their counterparts at the main office through their use of phone, chat and email. They select their communication style according to the value client centricity and emerge as a sub-system within the street office. In contrast, the execution team leads operate on the basis of the value ground reality: they do not primarily work at their desk, but move frequently between their desk and the freelancers' space in order to catch up on the freelancers' work status. Consequently, the execution team emerges as a sub-system with a different structure and communication style: this team reverts back on emails significantly more slowly than their office peers, they are not available on chat for spontaneous informal communication and they are usually on the move when called on their mobile. Their system is attuned to interaction with freelancers, and not with the rest of the organisation. I illustrated in Chapter 5 how this differentiation is expressed in the self-description of street office employees as the "simple people". The street office orientates along the value ground reality, rather than client centricity. This is apparent in Rohan's answer to the question of where he wants to work in the future: "Here only. I like to talk 
and interact with my team, with my freelancers, the supervisors. I do not like talking with my manager [at the main office]."

At the street office, the ground reality is the most relevant selection criteria. The execution team leads select their communication on the basis of what will be understood by the freelancers, rather than colleagues at the main office. The notion of these different sub-systems was voiced by Mudra, whose job was to distribute upcoming projects amongst the execution team leads, and who did not interact with freelancers:

I don't interact with these people [the execution team leads], I don't feel comfortable when they are rushing in and out. I think we should be sitting in the main office as well. I feel much more closer to the mind-set of the people in the main office [...] I have already asked the manager why we are here, and even wrote an email. But well, these guys [waves indifferently around addressing the office area] share a good rapport with the freelance side people, so they are fine. But I don't. I don't talk to these people; I only talk to her [points to the colleague behind her, who joined with her together].

Her statement not only communicates the boundary between the two subsystems at the street office, which operate on the basis of different values, but her desire to move to the main office also implies that the client-centric subsystem she belongs to in the street office carries less prestige than that which is associated with the execution team leads. The client centricity scale is reversed at the street office, as the ground reality is the primary differentiation criterion (Figure 11 - next page).

\subsection{Lunchmates and batchmates: Informal bridges across the office}

In accordance with Luhmann's Systems Theory, I have elaborated on the internal differentiation of the organisational system into hierarchically and functionally distinct sub-systems. Luhmann, however, distinguishes another stable category with the potential to develop into further sub-systems: "conform/deviant" sub-systems, which include official/unofficial or formal/informal sub-systems (Luhmann 1995a: 190). I touched on this category in Chapter 4 (Section 4.3.4), through the example of continued communication between ex-employees and their former colleagues who still work at Advice Company. While such sub-systems emerge from within the boundaries of Advice Com- 
Figure 11: Reversed values at the street office

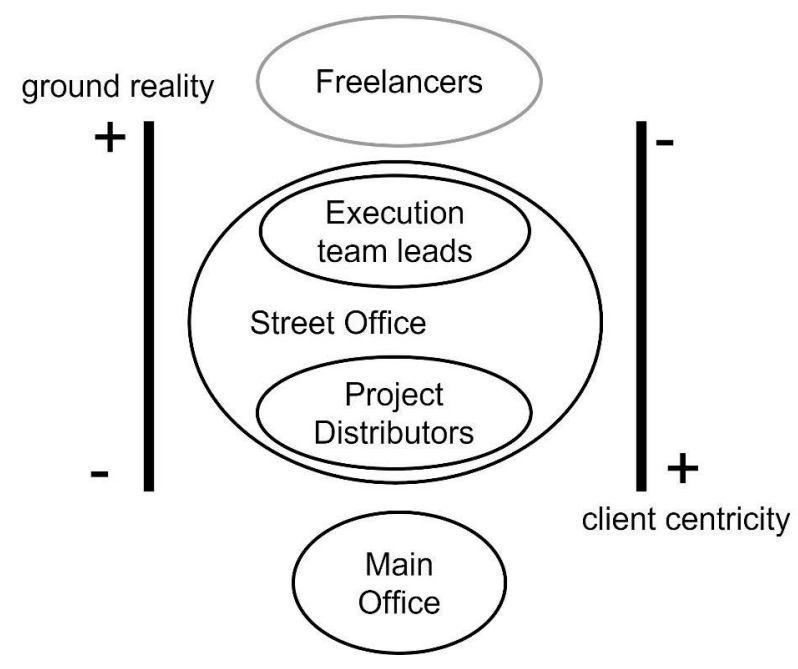

pany, they can proliferate beyond the system and even "last longer than the initial one" (ibid.: 189). In this section, I will trace the emergence of (relatively) permanent sub-systems that cannot be categorised as hierarchical or functional. For this analysis, the clear set-up of the city office and the limited size of the offshore support team (of 120 people) who work on the ground floor enabled an understanding of not only the physical distribution and boundaries of the formal sub-teams, but also the existence of both formal and informal communication systems. I analyse my interlocutors' informal social networks by tracing the persons they accompanied on lunch breaks, chai breaks and other non-work-related situations. For the purposes of triangulation, these observations are matched with interlocutor's self-assessments, which are obtained from individual ego-centred network diagrams. Through this data, a "webwork diagram", as I like to call it, is created. This diagram represents the lived communication praxis at the city office and reveals another layer of the perceived, emic perspective of the organisational structure in this office. 


\subsection{1. "We don't talk much": Perceptions and lived praxis}

Despite the absence of walls in the city office, the employees' descriptions of the office align with the organisational structure and the neatly divided seating arrangements of the different teams (Section 6.1.2). My interlocutors described their interactions with members of other teams as "we don't talk much". During my research phase at the city office a new project was started on the basis of a management initiative that aimed at creating a knowledge sharing platform to foster exchange among employees. The fact that such a project was initiated shows that the management team at the city office shared a similar impression of limited cross-team interaction, as my interlocutors expressed.

Advice Company furthermore used other formats to stimulate interaction amongst employees beyond the primarily subject of work-related tasks. Apart from a yearly cricket tournament, the "Presentation League" was launched in the main office and the city office. Colleagues from different parts of the organisation were invited to compete in mixed teams and asked to create presentations addressing difficult or controversial topics, as pre-selected by the jury of senior managers. These topics were socially controversial and unrelated to client projects; for example, they included suggesting a business plan for a gay marriage website in India and developing an advertising campaign for pharmacy chains intending to sell marijuana at their outlets. The Presentation League was designed to foster discussion amongst the temporary teams. Beyond this initiative, a team comprised of members from all departments organised office leisure activities, such as the Friday afternoon radio hour, during which popular music would be played across the office floor. Although these organisation-driven formats existed and I was able to observe the interactions they triggered, the employees' perception of the formal divide persisted.

Yet, when mapping the persons with whom my interlocutors spent their lunch and chai breaks, a strikingly different picture emerged, characterised by strong interlinkages not only within each employees' team, but also across the entire office. Furthermore, the lunch groups mirrored neither the formal team structures nor the organisational hierarchy (that divided team leads and team members) or the job type division between the standard and embedded teams (Figure 12).

These emergent sub-systems remained stable for several months or even years, as members met regularly for lunch at a table in the cafeteria or a col- 
Figure 12: Lunch group connections at the city office

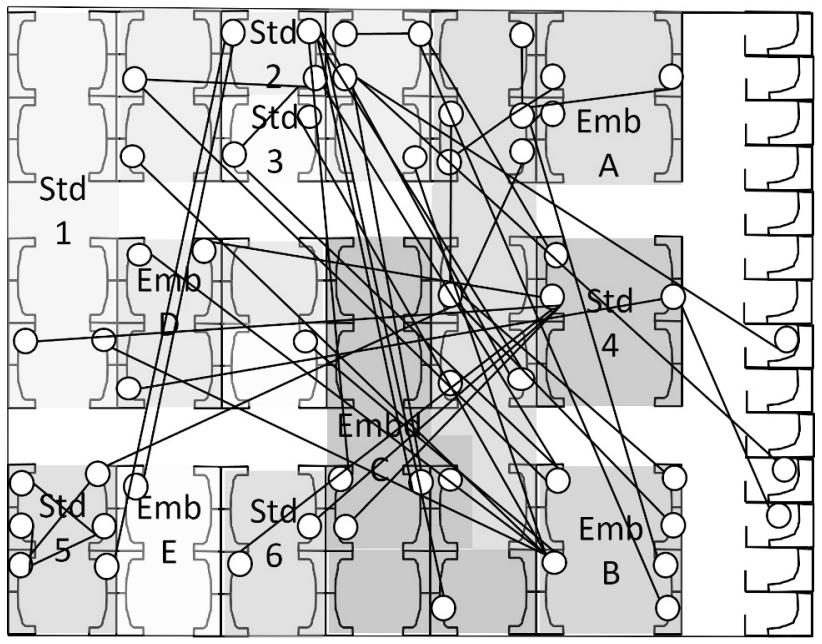

league's desk. When decisions were triggered to place a takeaway order from one of the numerous restaurants nearby, initiators would either start a multiwindow chat session or directly approach the groupmates to ask for their order, then would fetch the bags from reception once the food arrived. When all had eaten, washed their hands and potentially stowed their tiffins back in their bags, the group would gather again to go outside the office for a short walk through the compound or around the corner to the kiosk for mukhwas (anise flavoured breathe fresheners) or chocolates. In the afternoon, some of the groups would gather again for a chai break - each group had their favourite chai stall on the main road.

\subsubsection{Patterns of emerging informational sub-systems}

The lunch groups represented a rather egalitarian structure that diverged from the formal differentiation categories and sub-systems. The membership criteria for the lunch groups varied, but one rather surprising criteria became salient: "batchmateship" - a term frequently used in Advice Company to refer to colleagues who joined the organisation at the same time. A large proportion of the lunch groups consisted of colleagues who had joined the organisation 
at the same time. Some of them had been at the same college and were hired via the intense campus recruitment process. Many of them shared the experience of having "lost" collegemates in each new decision-making round, until they - the two or three "chosen ones" - finally "made it" together. Even when the group members were not from the same college, belonging to the same "batch" and having jointly gone through the process of becoming a member of the organisation at the same time was described as a strong bonding experience that could form persistent informal groups on a level that was independent from the organisational structure.

Sonali and Ameya demonstrated the important link that batchmateship could provide between new colleagues. Sonali, from the city office, asked me which teams I had accompanied at the main office. When I began to name them, Sonali instantly prompted the name of Ameya, who had been her batchmate five years prior. She could recall the team Ameya had been in and knew where she was now. When I was back at the main office, I mentioned to Ameya that I had accompanied a former batchmate of hers at the city office. Accordingly, she did not even have to think for a second to come up with Sonali's name.

But the lunch groups not only evolved around batchmateship; several groups consisted of members from former team structures that had originated in the early days, when the department was known as the "fun place". While the formal sub-systems had dissolved through organisational restructuring and members moving to other teams, the informal sub-system of the team remained intact across teams, job types and hierarchy levels. In one remarkable example, the sub-system even persisted across offices. I accompanied sheeba in the main office and asked her about a laminated group picture at her desk. She told me enthusiastically about her old team at the city office and how it had been closed three years ago. But most of the former members had taken new jobs in other teams at the city office and she desperately wanted a job at the location. A few months later, she managed to get a placement at the city office, and during my fieldwork there, I recognised several of the colleagues in her photo when I accompanied her to lunch.

\subsubsection{Lunch groups: A totally independent sub-system?}

These lunch groups existed as a stable category of emergent sub-systems in parallel to the functional and hierarchical differentiations. They emerged from shared values - either through members' shared joining experience or work 
experience in a "distant past" - and were independent (in their communication structure) from the organisational value system of client centricity. While the communication in these sub-systems did not function or process information according to the organisational framework, the lunch group subsystems nonetheless reinforced the organisational boundaries, as they could not exist without the organisation. The lunch groups could also be seen as networks - forms of social order consisting of reflexive contacts that could emerge within or between social systems (Holzer 2010: 163) - and they repeated the system/environmental differentiation. Thus, the lunch groups represented an informal network within the organisation that were restricted by organisational membership (ibid.: 158).

The independence of lunch groups from the formal structure of the organisation became salient when I initially tried to set up focus groups to discuss topics of misunderstanding. I discussed in Chapter 3 (Section 3.4) why focus groups did not prove helpful for generating data. However, before I refrained from using that method I had the idea of letting my interlocutors decide the persons with whom they would like to have the discussion; one of my thoughts was that lunch groupmates might provide a sound basis for a discussion group. But Ameya opted for a different group, consisting of colleagues in her work team. When I pointed out that we could also set up a group with her lunchmates, she answered: "You know, these guys are my pals, I am not sure if I want to discuss work items with them." Ameya definitely interacted with some of her lunchmates with respect to work-related issues, but she perceived the explicit connection of this lunch sub-system with discussion of her work sub-system as inappropriate. For her, the focus group event did not constitute a condition in which she was willing to transfer information and resources from the informal to the formal context (Holzer 2010: 162).

In other situations, however, a transfer of interaction across the formal/informal divide occurred. As informal as the lunch groups might have been perceived, they certainly had an influence on the formal structures within the organisation. In the interviews, my interlocutors assessed such informal connections as potentially unfair, since friendly contact with a member in the group might result in favouritism with respect to promotion opportunities. When I accompanied Sonali, from the standard team, I observed such an example. Joel, a team lead, who sometimes joined her lunch group, contacted her via the chat program and told her about an upcoming job opportunity in one of the embedded teams. He asked if she would be interested in applying for it. The communication medium he chose indicates 
the semi-formal nature of the conversation. Furthermore, the adjustments made to accommodate the situation necessitated "switching" between contexts (Mische and White 1998: 710). By choosing the chat program, Joel was able to contact her without revealing the content of his message to others, as he would have done had he simply come by her desk. On the other hand, he did not choose to communicate via the more formal email channel, nor did he call her to his cubicle or a meeting room. But he also did not choose to raise the topic over the lunch break, when it would have fallen into the informal sphere. The chat program allowed for the semi-formal style of informing her of this job opportunity without raising the attention of other (also potentially eligible and interested) teammates.

\subsection{Concluding remarks on the organisational system}

Part I has analysed Advice Company as a social system on the basis of Niklas Luhmann's Systems Theory. In Chapter 4, I illustrated how complex organisations shape and underline their boundaries with the environment, drawing on the example of the elaborate entry procedures at the main office. Advice Company further differentiates itself from its environment in its communication with the most relevant environmental systems: clients and freelancers. These systems are at opposite extremes of the client centricity scale, despite being attended to through structurally similar boundaries. In Chapter 5, I demonstrated how client centricity constitutes a superior value in the organisational system, not only with respect to the organisation's external boundaries, but also in its internal hierarchy.

Through a comparison of the three offices' respective access procedures, equipment and atmosphere, it became clear that the offices could be placed on a continuum of client centricity. The main office is the most client-centric location, the city office occupies a middle position on the continuum and the street office is the least client-centric of the three. The analysis of office atmosphere further revealed the oppositional value of the organisation's guiding difference that runs as a counter-current to client centricity: the ground reality.

Chapter 6 carved out the organisation's further differentiation into emergent sub-systems on the basis of job type hierarchies that follow the client centricity paradigm, with jobs with the most direct client interaction at the top of the hierarchy. In the street office, however, the internal differentiation 
orientates on the value ground reality and hence reverses the client centricity scale.

Following Luhmann's categories of conform/deviant system in system differentiation, I visualised (in Section 6.2) the emergence of lunch groups as informal sub-systems. These sub-systems provide communication bridges across the organisation's formal sub-systems and enable translocal ties between them. One hypothesis is that employees' shared history as batchmates or (ex-)teammates creates an egalitarian setting that allows them to bridge the intra-organisational borders.

Part I of this book has demonstrated the structures of the client-centric organisation, in which closeness to the client is he highest value and, consequently, associated with prestige and power. The analysis has illustrated the leading influence of the social system on the communication and behaviour of its members, who act in accordance with the client centricity value paradigm, independent of their office location or functional proximity to clients. Furthermore, the analysis has shown that the emergence of sub-systems is dependent on the organisation's guiding difference client centricity/ground reality. The physical proximity of members in the open plan office setting does not suffice for the emergence of sub-systems, as internal differentiation (predominantly) occurs on the basis of client centricity. The value ground reality seems to counterbalance these structures and sometimes even invert the client-centric orientation of the organisation, although it remains an implicitly expressed opposing value. In Part II of this book, the focus will shift from the structure of Advice Company to its inter- and intra-system interactions. I will demonstrate that working misunderstandings repeat these organisational structures and, at the same time, strengthen them via the system's autopoiesis. 
Part II: Working Misunderstandings 



\section{Working Misunderstandings}

The first part of this work showed how Advice Company is structured as a social system aligned with the guiding difference client centricity/ground reality. This second part focuses on working misunderstandings in the context of this organisational system. Chapter 2 (Section 2.4.2) illustrated how misunderstandings can be regarded from a systems-theoretical perspective as inevitable components of communication and thus important building blocks of a social system. When communication occurs across the boundaries of social systems, the different selection processes in each system are likely to result in misunderstandings. Therefore, misunderstandings can be regarded as a communicative symptom of system differentiation, through which the boundaries between a social system and its environment become salient.

The hypothesis here is that interaction across social systems functions not only despite, but also as a result of these misunderstandings, which arise from the different selections at play in each system. In the following, I will show how a sending system selects information and an utterance and how the receiving system selects its understanding in order to reproduce the information (and therefore recontextualise it) within its own boundaries (Gershon 2005: 103).

Part II commences in Chapter 7 with a review of anthropologists' and other scholars' use of the concept of working misunderstanding ${ }^{1}$. Based on this literature review, I will propose a framework of analytical categories that differentiates between the locus (involved parties) and modus (level of intentionality) of a working misunderstanding: the "L/M quadrant". This framework will then be applied in an analysis of the various working misunderstandings in the organisational system of Advice Company, drawing on several case studies. The case studies relate to the central service commodity of Advice Company: the client project. This main service commodity ensures the survival of the organisation; hence, it must be processed by all of the organisational sub-systems. For this reason, the client project constitutes the main communication operation that reinforces the structures it enables to exist. Section 7.2 introduces the client project as an analytical entity and explores its development across the organisation. 
Chapters 8, 9 and 10 are aligned with the L/M quadrant and illustrate how the four categories of working misunderstandings are encountered in connection with the client project (Figure 13).

Figure 13: L/M quadrant and chapter outline of Part II

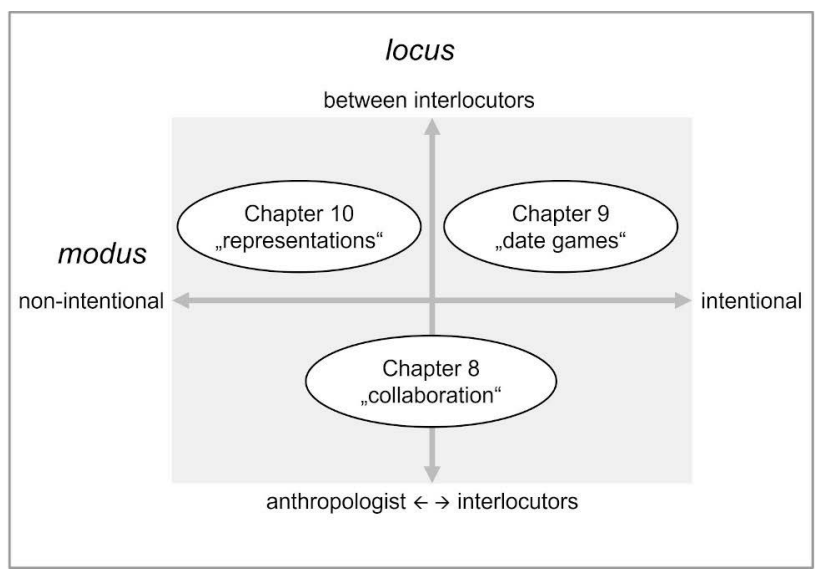

Chapter 8 explores a working misunderstanding between the employees and myself around the notion of "collaboration" (locus interlocutor $\leftrightarrow$ anthropologist). It describes how my understanding of project collaboration - despite differing substantially from the concept as practiced by the employees initially invoked no dissonance and retraces the point at which the working misunderstanding became salient. Furthermore, it shows how I consequently reshaped both the data collection focus and my actions to comply with the notions of collaboration at play in the organisation. In Section 8.1, I illustrate the transformation of this working misunderstanding from a non-intentional modus to an intentional modus over the course of fieldwork, re-shaping my strategy for knowledge management. This case is positioned within the suggested framework under the modus of intentional working misunderstandings - a seemingly marginalised and/or underreflected category in ethnographic accounts (Section 8.2).

Chapters 9 and 10 focus on the working misunderstandings between my interlocutors. Chapter 9 is located within the modus of intentional working misunderstandings and describes a working misunderstanding I call the "date games". It refers to the project planning phase, during which the 
different sub-systems seek to optimise intersystem collaboration by reinterpreting delivery dates. This "bargaining game", as project coordinator Asif called it, assumes that information about delivery dates has the purpose of ensuring timely project completion. In this game, however, it is not necessarily the actual delivery dates that are communicated between teams, but those that are assumed to provoke the desired reaction within the other subsystem. This situation of double contingency can be connected to intentional working misunderstandings.

Chapter 10 suggests that a client project may give rise to an unintentional working misunderstanding, and traces different views on a project as it moves along the project development process. The cases show that a project changes in form and status, from a client's strategic need for decision-making to executable work tasks, detailed presentations and, ultimately, a final strategy. Hence, each project can be seen as a working misunderstanding in its unique "working" quality, which allows each sub-system to attach systemspecific meaning to it. As I will demonstrate, this underlying "working" substance of a project gives opacity to the guiding difference of client centricity to which all communication within Advice Company connects its project understanding. 



\section{Working Misunderstandings}

\subsection{Working misunderstandings and ethnographic insight}

The term "working misunderstanding", in the context of ethnographic research, was first coined by Paul Bohannan in his analysis of colonialism in Africa.

In an African colony, then, the political and economic situation was assessed by the European rulers in terms of European culture; the same situation was assessed by Africans in terms of their various African cultures. Their common heritage and their common humanity assured that for some matters the two evaluations were complementary. Just as surely their separate histories led them to view other matters divergently. [...] Such is the nature of the "working misunderstanding". [...] There were two sides and neither really knew the "codes" - the connotations of word and deed - in which the other group perceived the situation, valued it, communicated about it, and acted. (Bohannan 1964: 12-13)

According to Bohannan, the political and economic structure of colonialism was possible because of the differing interpretations (i.e. selections of understanding) of the situation by the colonisers and the colonised, and remained "working" as long as the two systems were kept apart (ibid.: 25). Or, as Marshall Sahlins put it more precisely: „We have to deal rather with a parallel encoding [...] as a "working misunderstanding." It is a sort of symbolic serendipity, or at least a congruent attribution from two different cultural orders of a special meaningful value to the same event." (Sahlins 1982: 82)

Hence, a working misunderstanding arises when at least two social systems interact with each other on the basis of a common situation, term or activity. Both systems interpret the situation (i.e. select their understanding of it) in terms of their system-specific context, which might fundamentally differ 
from the other's. But the situation can be re-contextualised so that it "makes sense" within the realms of one's own system (Gershon 2005: 103) and this enables a continuity of interaction. Interaction across social systems functions not only despite, but also because of these working misunderstandings. They can be seen as the bridge between systems that enables uninterrupted (i.e. successful) interaction between them, and hence intact and "working" communication.

Such working misunderstandings have been employed by a number of scholars, within both anthropology (Reed 2006, Wijsen and Tanner 2008, Watkins and Swidler 2013, Cole 2014, Losonczy and Mesturini Cappo 2014, Dorward 1974) and other disciplines, such as sociology (Jaffee 2012), law (Chen-Wishart 2013) and history (Spear 2003, Iliffe 1979). The term "productive misunderstanding" in ethnographic studies is almost synonymous (Gershon 2005, Tsing 2005, Livingston 2007, Monteiro and Keating 2009), as is "malentendu productif", which is a direct translation of the term in French (Nadége 2007: 34, Papinot 2007). Other scholars have drawn on the concept of structured misunderstandings (Servais and Servais 2009) - "malentendu bien entendu" (La Cecla 2002) - or have not used a specified term to illustrate the role of misunderstanding as a means of supporting successful social interaction (Durrenberger 1975, Fabian 1995). Guido Sprenger (2016) proposes a differentiation of structured and unstructured misunderstandings, with the former based on the existence of a shared mode of communication a term or set of terms with partial semantic overlap that is used by both parties. Unstructured misunderstandings, in contrast, rely on serendipity, as illustrated by Sahlins (1982).

These accounts of working misunderstandings illustrate the positive nature of misunderstandings and their potential contribution to successful social interaction. Application of the concept, however, remains far from coherent and has often lacked analytical direction. Working misunderstandings are obviously a long-lasting topic of academic interest, as they have featured in publications for the past 50 years. Yet no effort has been made to structure the discourse on analytical positions pertaining to working misunderstandings; here, I propose a starting point. 


\subsection{Working misunderstandings as an analytical category}

To approach a common ground for discussion of working misunderstandings as an analytical category for ethnographic insight I will draw on a selection of the works mentioned above and derive from these accounts the two analytical dimensions on which the suggested quadrant typology builds on: locus and modus.

The locus dimension differentiates working misunderstandings into those arising between interlocutors and those encountered between the anthropologist and interlocutors. I will illustrate this dimension in the following section (7.2.1) using three examples from the existing body of ethnographic studies. Section 7.2.2 will address the modus dimension, which defines working misunderstandings along the lines of intentionality and non-intentionality, on the basis of further ethnographic examples from the literature. Both dimensions (locus and modus) will then be combined into a quadrant typology model, onto which the current body of literature will be structured (Section 7.2.3).

\subsubsection{The locus: Misunderstandings amongst interlocutors or between interlocutors and the anthropologist}

Watkins and Swidler (2013) illustrate, in their work, how the different parties involved in HIV prevention (donors, brokers and villagers) operate on a narrow set of programme labels - or "themes that make everyone happy" that enable all agents to attach different meaning. Intervention programmes aiming at "fighting stigma", for example, can appeal to religiously motivated donors, as they enable HIV intervention without mentioning sexual protection. Of course, for Malawian communities, "fighting stigma" merely refers to reciprocal obligations to kin. Although the theme means something different to each of the involved parties, all are able to work together under it. The common theme allows parties to collaborate without needing to confront the different meanings attached to the same words (ibid.: 203).

Similarly, Anna Tsing collected stories of the different key players involved in a successful anti-logging campaign in a Meratus village in Indonesia. In this process, she realised that all parties seemed to describe different events, corresponding to their differing commitments to nature. It was not despite, but because of the misunderstandings between village elders, provincial nature lovers and national environmental activists that they were able to collabo- 
rate successfully and accomplish the campaign's goal of removing the logging company from the village (Tsing 2005: $245 \mathrm{ff}$ ).

A further ethnographic example of the productivity of misunderstandings is provided by Marko Monteiro and Elisabeth Keating (2009), who collaborated in an interdisciplinary cancer research team with scientists from fields ranging from computer science and biomedical engineering to applied mathematics and medicine. By tracing the communication at the team's weekly meetings, they illustrated how strategies such as the "eyeball norm" (presenting data in such a way that it looks plausible to everyone) were used to facilitate successful collaboration despite a lack of shared understanding across the disciplines (ibid.: 9).

These three studies refer to the same locus of working misunderstanding, as they each analyse how a working misunderstanding supports interaction between interlocutors. They illustrate the successful cooperation of different parties despite incongruent - or even conflicting - pre-dispositions and worldviews. The working misunderstandings discussed in these three studies relate to topics the researchers were able to examine without being part of the misunderstanding, themselves. The authors seemed to have no strong ascriptions to the situation of misunderstanding, and no conflicting understanding that was salient in the analysis.

But working misunderstandings can also arise between an anthropologist and his or her interlocutors. The detection of misunderstandings, in general, is neither surprising nor rare - it is part and parcel of fieldwork and is of value to the anthropologist, as it renders the perception that he or she is in dialogue with agents of a group with a different conceptual framework. The working misunderstanding, however, provides an additional level of insight, as it not only highlights the distinctiveness of the other party's understanding, but it also reveals layers of similarity, as the actions that result from one party's understanding are similar or at least comprehensible to that of the other party, despite the differing understandings. This is why misunderstandings can go unnoticed until a behaviour reveals the difference. The "working" aspect describes the point to which a more or less superficial congruence between the anthropologist's and the interlocutors' ascriptions exists. Such working misunderstandings have seldom been analysed in the literature, and my own case, analysed in Chapter 8, will contribute to filling that gap. The following three studies have, however, already addressed the issue.

Johannes Fabian (1995) describes how he assumed the Swahili term muzungu applied only to a white man when one of his interlocutors told him 
about a muzungu who had joined a religious movement in Zimbabwe. Only years later did he realise that it was the female African-American anthropologist Benetta Jules-Rosette to whom his interlocutor had been referring to as a muzungu in the interview (ibid.: 43). The term muzungu, for the informant, referred to a non-African, while Fabian inferred that it described a white, male person, despite being aware that the Swahili language does not mark gender. But, as Fabian points out, ethnographers must accept misunderstandings and "settle for a version we can live with" (ibid.: 44) in order to continue communication and allow the cross-system interaction to function.

In a methodological reflection on the application of photo-elicitation interviews, Christian Papinot (2007) describes how a misunderstanding between himself and his interlocutors on the meaning of photos proved productive. He had taken a series of close-up photographs of decoratively painted inscriptions and motifs on public transport buses in northern Madagascar, with the intention of utilising these photographs in his interviews to trace his interlocutors' interpretations of the motifs. But his understanding of the photos as a supportive tool of enquiry differed significantly from his interlocutors' understanding of them. His use of photographs in the interviews collided with the Malagasy social definition of a photograph (Papinot 2007: 83). Consequently, the images did not lead to interpretations of the decorations, as Papinot had expected; rather, the close-up, seemingly truncated images were perceived as an invitation to identify the driver of the vehicles. However, this misunderstanding of the purpose of the photos was productive, as it allowed for a conversation that led to the revelation of a connection between the decorative elements and a rivalry that was occurring between vehicle drivers (ibid.: 84).

A final example relates to Anna Tsing's reflection that understandings of a forest can be social, rather than naturalist, which she learned in the course of her fieldwork. She realised that her view of the Meratus forests was steered from a naturalist perspective, which led her to appreciate the variety of species and the forest views from a mountain ridge. Her interlocutors' understanding of the forest, however, was one in which "individuals and households traced their histories: House posts resprouted into trees. Forest trees grew back from old swiddens." (2005: xi). Here, the anthropologist became part of the discourse, as her understanding of a concept, term or situation differed from that of her interlocutors. 


\subsubsection{The modus: Tracing the intentional/non-intentional}

The examples from the literature discussed in Section 7.2.1 centred on the analysis of a working misunderstanding between interlocutors or - in the latter case - between an anthropologist and interlocutors. But despite their difference in locus, one can locate a commonality in each of the working misunderstandings: their non-intentionality (modus). The working misunderstandings happened unconsciously and were unintended - at least, this is my assessment of the situations on the basis of the information provided in the literature. Neither of the involved parties seemed to have insight into the other party's ascriptions, and they did not actively shape their actions to maintain the misunderstandings working.

Working misunderstandings, however, are not always kept "working" maintained solely on the basis of an incidental, undiscovered and semantic overlap across interacting systems. While Sahlins (1982) argues, in his account of Captain Cook, that a working misunderstanding occurred between Cook and the Hawaiians - explaining that Cook's murder was not a necessary consequence but a possible consequence of the working misunderstanding he primarily wished to explain the behaviour of the Hawaiians, who perceived Captain Cook's behaviour as fitting well into their context (Reed 2006: 157). Isaac Reed instead argues that Cook might have understood the role in which the Hawaiians saw him very well and adjusted his behaviour accordingly, in order to avoid being unmasked as a human being (e.g. by avoiding women, unlike his crew members). In order to maintain the misunderstanding working, "each side played certain roles in the other sides drama" (ibid.: 158).

Similarly, in her study of Malagasy women who are married to French men, Jennifer Cole (2014) illustrates how these women strategically play on ambiguities in Malagasy kinship as a working misunderstanding in order to maintain the complex interactions between the French and Malagasy family systems:

She claimed that the French term sœur [sister] and the Malagasy term rahavavy [sister] referred to the same semantic field, even though she knew that rahavavy covered a wider range of kin than her husband would have recognised as sœur. She built a working mis/understanding premised on the gap between Malagasy and French definitions of the term sister to smuggle in - literally - a relative whom she deemed important but whom Pierre [her 
husband] would almost certainly not have counted. (ibid.: 541, emphasis in original)

A party's ability to actively play on such unarticulated ambiguities and semantic overlaps is a particularly interesting aspect of analysis in relation to working misunderstandings, as this ability requires a certain level of knowledge of the other party and conscious shaping of one's actions to bring them in line with the other's expectations.

Anne-Marie Losonczy and Silvia Mesturini Cappo illustrate this in their study of two Ayahuasca shamans in Iquitos (Peru): one of them failed to attract occidental clients due to his inability to play on the working misunderstanding of shamanism between local/mestizo concepts and Western ideas, which the other apparently did very well, due to his experience of travelling through Europe and his frequent contact with occidental tourists and apprentices (2014: 124-26). The latter shaman was able to actively shape his actions to make them more similar to ascriptions of shamanism between the two social systems, allowing the misunderstanding to remain working and preventing dissonance with his European apprentices.

The common notion of misunderstandings sees them as unintentional and contingent. The ethnographic accounts in this section, however, show a certain level of intentionality on the agents' part to keep the misunderstanding working by leveraging opacities in the interacting systems. I therefore suggest to structure working misunderstandings along a second dimension that differentiates between notions of intentionality and non-intentionality. This second analytical dimension of the modus will be applied to case studies of client projects at Advice Company in the context of unintentional (see Chapter 8, Section 8.1 and Chapter 10) and intentional (see Chapter 8 , Section 8.2 and Chapter 9) working misunderstandings.

\subsubsection{Towards a framework of working misunderstandings}

I have illustrated how the analytical category of working misunderstandings can be separated into two dimensions: the locus and the modus. These dimensions, which derive from existing applications of working misunderstandings in ethnographic analysis, are proposed as a categorical orientation to structure the existing body of research on misunderstandings and to foster further discourse on this topic. To account for the interdependencies of the analytical dimensions while retaining a certain level of lucidity, I suggest their arrange- 
ment into a quadrant, along which the examples discussed can be allocated (notwithstanding the risk of oversimplification) (Figure 14).

Figure 14: The L/M quandrant of working misunderstandings

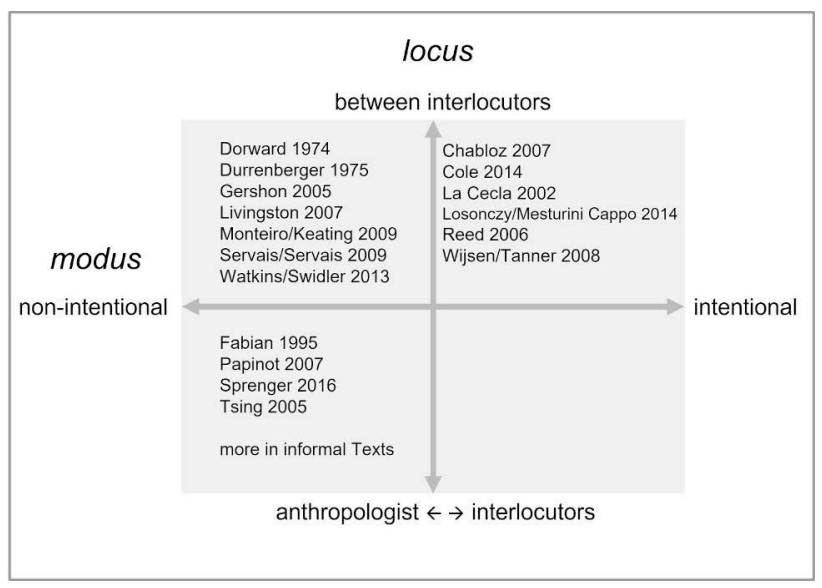

From the several accounts of working misunderstandings, it is apparent that, in general, the locus anthropologist $\leftrightarrow$ interlocutor is an underrepresented category in ethnographic literature. Accounts reflecting the intentional modi of working misunderstandings between the anthropologist and his or her interlocutors constitute a seemingly marginalised category. This category refers to working misunderstandings in which the anthropologist is (at least partially) aware of the differing ascriptions of his or her interlocutors, and actively shapes his/her behaviour to keep the misunderstanding working. Such a situation can of course also occur in the opposite direction, with interlocutors modifying their actions to comply with the anthropologist's different understanding. Uncovering such working misunderstandings is difficult, and misunderstandings of this type were not presented in the literature review.

In the following chapters, I will apply each of the four categories of working misunderstandings and illustrate the potential of the modus and locus as analytical dimensions. By reflecting on an intentional working misunderstanding between the interlocutors and myself, I will address the identified gap in the analysis of working misunderstandings. 


\subsection{The client project as a service commodity}

Section 7.3 takes the client project as the central subject of analysis, following the project development process through the organisation and situating the development steps along the client centricity scale.

\subsubsection{Following the project's process}

Advice Company generates a significant amount of revenue by providing advice and consultancy in relation to clients' specific strategic decisions of their clients. When a client representative approaches a member of the consulting team in order to request help in resolving an issue or question, or when Advice Company pro-actively offers follow-up services on a completed project, an iterative phase of project proposals, negotiations and refinements follows. When Advice Company wins the "pitch" of project proposals over competitors, the project is officially ordered and a contract is signed. In a teleconference between Advice Company consultants and the client, the project scope, expectations and details are discussed. These meetings are often supported by documentation that is sent by the client in the form of a presentation or text file prior to the meeting. This project briefing represents the central communication over the organisational boundary, with the consultants serving as the boundary communication specialists.

Once a project is requested and the briefing is complete, the consultants conceptualise the project and plan the expected degree of involvement from the executing teams. Through internal briefings, the project is handed over to the project coordination team with specific timeline expectations. The project coordinators organise further briefing meetings with the execution team leads in order to inform them of the tasks and required delivery dates. Once the execution teams complete their tasks, the preparation teams transform the information into presentation files and other serviceable outputs. These raw presentations are then analysed by the client consulting teams, who select and condense the information. Through the final shaping of the slides and phrasing of the conclusion's wording, the presentation file is transformed into a strategic advice report. This report is then delivered to the client in the form of a performed presentation talk, often at the client's premises. Figure 15 illustrates this full client project development process. 
Figure 15: The project development process

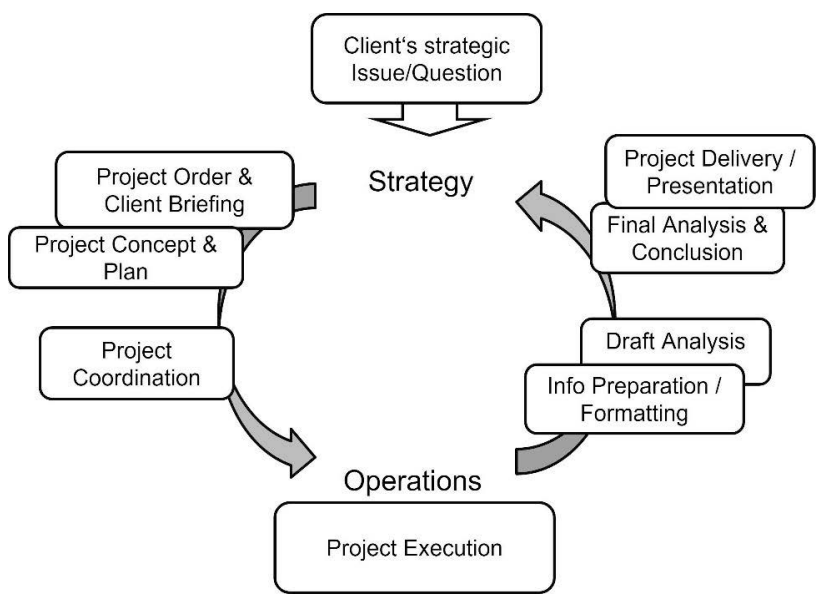

\subsubsection{The client centricity scale and the project development process}

To set the context for the following chapters and to connect the client project to the analysis performed in Part I of this book, I will position the client project and the project development process within Advice Company's organisational orientation along the value client centricity, across the three offices (see Chapter 5).

Once a project is confirmed by a client, the sequence of work tasks starts with the client's project briefing. The client project tasks are shared across all three offices of Advice Company in the city and allocated according to the client centricity continuum. The tasks most directly associated with boundary work to the client system are performed at the main office, while the operational activity around the project occurs at the street office - the location associated with the ground reality. At the city office, the work of the execution teams is transformed into a format that can be processed by the consulting team for the project report; therefore, this work occupies a middle position along the client centricity scale. The final project report is produced and delivered by the client consultants at the main office. The initial tasks of the project and the final actions preceding the delivery are not only the most 
directly connected to the continuity of the organisation, but also the most directly connected to the client.

The concluding step - the project delivery in the form of a presentation is often performed at the client's office. This step represents, both physically and structurally, the closest interaction between Advice Company and the client. In the client-centric organisation, this task is associated with high prestige. Stories of how client presentations went, how challenging questions from clients were mastered, what feedback was received and what funny interactions occurred with clients' representatives are told aloud in the office and even retold for several days after the event. I witnessed numerous conversations amongst client consultants in which references to client meetings were given, demonstrating their high prestige.

Figure 16 illustrates the project process, including the office in which each task is performed. Relating this classification to the structural set-up of the organisation (with its sub-systems differentiated according to the value client centricity), the strategic work aligns to client centricity while the operational work aligns to the ground reality:

Figure 16: Project process and offices

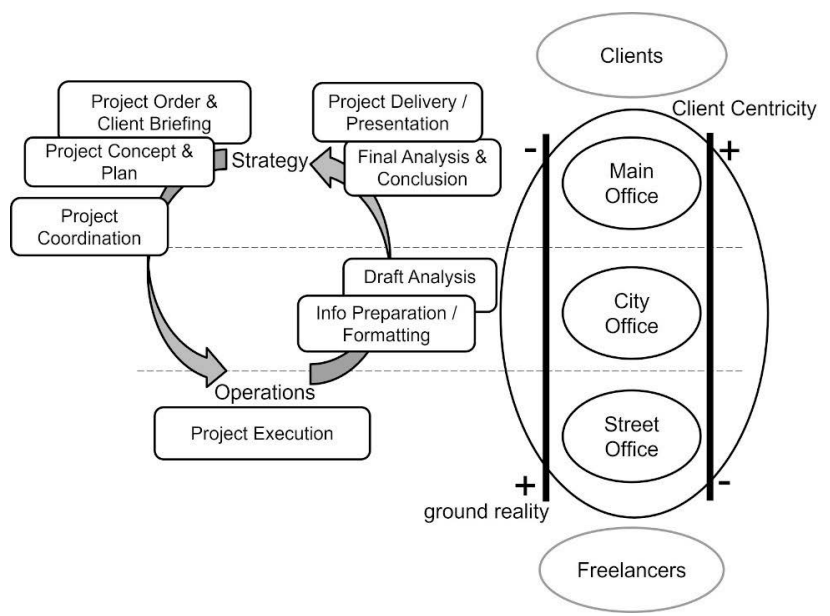

The tasks that are directly associated with generating revenue and a strategic, aggregated form of information (project briefing, planning, delivering the final conclusion and crafting advice) are conducted at the main 
office, while the work completed in the street office (by the less prestigious roles) is framed in opposition to crafting strategy. The project development process can therefore also be understood as running from client centricity to ground reality and back again. In this context, the challenging role of the project coordinators is particularly apparent, as these employees are located at the main office yet they must bridge the significant chasm between strategy and operations - between the mutually exclusive values of the organisation's guiding difference client centricity/ground reality.

In Chapters 9 and 10 I will analyse how the individual sub-systems in the project development process organise their interactions for successful project delivery along intentional and unintentional working misunderstandings, which can be variously positioned within the $\mathrm{L} / \mathrm{M}$ quadrant proposed in this chapter. 


\section{Collaboration as a Working Misunderstanding}

Before focusing on the working misunderstandings between interlocutors, I will discuss in this chapter a working misunderstanding of the locus anthropologist $\leftrightarrow$ interlocutors; that is, between Advice Company's employees and myself. The working misunderstanding centred on disparate notions of "collaboration" in the context of project work, and it remained undetected and working for the first phase of my fieldwork. I will illustrate how the working misunderstanding shifted from the initially unintentional modus to an intentional one, through which I shaped my actions to comply with employees' understanding of "collaboration" (Figure 17).

Figure 17: Chapter allocation on the L/M quadrant

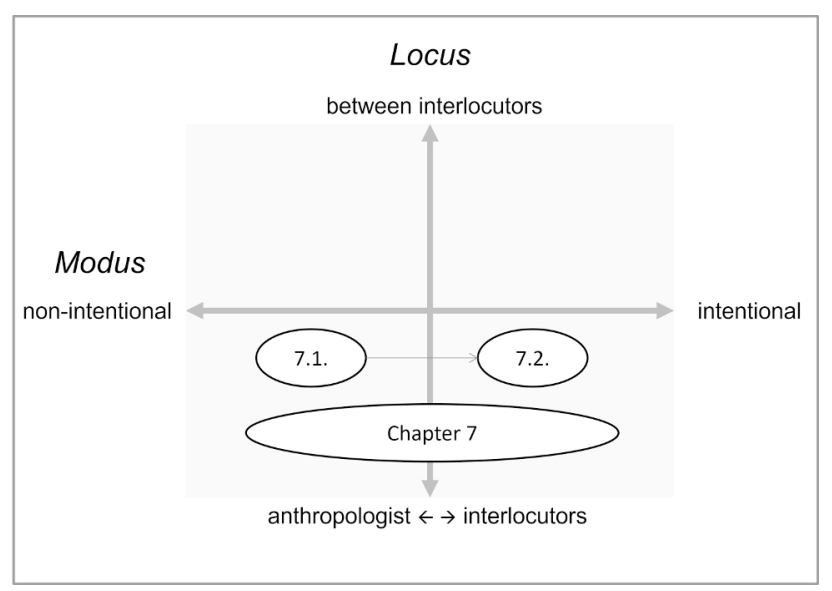




\subsection{Discovering "collaboration"}

The advice most frequently given in the Discover Advice Company training for new employees, which I attended very early in my fieldwork, was that employees should practise "boundaryless behaviour" in the office and "share information openly and freely across the organisation". Along with this advice came a few collaboration activation games, such as passing a sugar cube along a line of team members with chopsticks, in order to emphasise the importance of each team member to the success of the entire task (Section 3.4.2).

Indeed, the physical set-up of the main office stands in congruence with these messages, with its very stringent realisation of an open plan office with no walls or individual cabins for managers. All employees have exactly the same desks, regardless of their designation, and several semi-open chat corners or glass-walled meeting rooms provide interactive workspaces. The office space is very similar to the settings described in popular management books that are thought to facilitate a "culture of collaboration" (Rosen 2007: 116), and its open nature reinforces the notion of the organisation as a single system with values of transparency and egalitarianism. As shown in Section 4.5.1, the quotes of employees who saw the main office as a place for interacting and coordinating work also correspond with this notion, notwithstanding the other perceptions of pressure, distraction and fear that were voiced in connection with the main office's atmosphere.

These facts seemed to affirm my understanding of collaboration, which I thought was based on ideas of mutual knowledge exchange and common access to information. In my notion of collaboration, the central aim was therefore achieving the maximum amount of shared knowledge across collaborating parties within the organisation, as this would allow for the most beneficial work results (Squires and Van De Vanter 2013: 298). This understanding might have been partially related to my work experience as an IT professional managing software implementation, as my aim in this role was exactly to maximise the availability of information across organisations. Current discourses on collaboration in information systems research (ISR) place the topic in the context of cloud data storage technologies and collaboration software innovations (Kogan and Muller 2006, Li et al. 2012, Shah 2014).

During the early weeks of my research, as I accompanied the employees of several client consulting teams, I observed how the team members worked closely together on a task - sometimes literally, with two or three colleagues sitting together at the same desk, discussing and jointly developing presen- 
tations, documents and emails that would be sent to clients. They frequently sought advice from each other, and called across to the next line of desks if the colleague they needed to speak to was not sitting next to them. The interaction was marked by such high communication density and multi-tasking that the intensity of collaboration seemed to provoke questions about potential compromises on the individual's productivity (Heerwagen et al. 2004: 511). This relates back to the seemingly contradictory perception of the office atmosphere as one that both fostered coordination and distracted employees from "real work" (5.4.1).

In my next fieldwork phase, six weeks later, I accompanied colleagues on the project coordination teams, who had the task of supporting the client consultants by organising the work tasks that would be carried out by the freelancer teams ${ }^{1}$. When I asked the employees how they managed the different client projects that were handed to them from the consulting teams, my interlocutors patiently explained to me the various documents from which they drew the project information and in which formats they stored updates in shared databases to be reviewed by the other teams. To ensure compliance with the compulsory activities in the mandated sequence of the project management process, most colleagues maintained elaborate lists of 20 to 25 "to dos" for each of the projects they managed. All of these "to dos" centred on the exchange of project-related information or status updates on the project's progress, and this corresponded to the "boundaryless behaviour" requested by the managers in the initial training sessions. Furthermore, these actions seemed to confirm my ideas of collaboration, which encompassed the aim of achieving the maximum amount of information sharing along the project development process. I remained fully engaged, collecting very detailed data about the various technicalities of the project process and where each piece of information was stored. I did not perceive any dissonance between my notion of collaboration and the observed praxis in the office; nor did I realise that a working misunderstanding about collaboration was at play - the misunderstanding was apparently working very well.

It would remain working for a few weeks longer, until I accompanied Naveed, who happened to work on a project that I identified - after some time - as one that had been initiated by a client in the consulting team during my stint there. It had taken me almost half the day to realise that it was the tasks carried out by the freelancer teams. 
same project, due to the different information Naveed seemed to have about it relative to the information I had picked up from the first team. While the consulting team member had given me an elaborate account of the strategic intent and type of advice the client was looking for, Naveed fed me a summary version of it, focusing on the key data needed for the consulting team. The existence of an information gap between the consulting team and the project coordinators became most apparent in relation to timelines: Naveed seemed to have no information about the date by which the client expected the project to be finished and presented, but I recalled that such a date had been agreed over email. Naveed had only been given a deadline for delivering the tasks he was responsible for. For a while I wondered if I had simply done a poor job of taking notes on the facts of the project, mixing up clients' names or the relevant databases and tools used for collaboration. Despite assuring myself that this was not the case, I still assumed my notion of collaboration to be valid. I remained fully focused on discovering how the information on the project given to the first team could have been reduced and changed across only a 15-metre stretch of desks, despite standardised processes, databases and "to do" lists.

A few hours later I heard and saw Naveed give his main contact at the street office, who coordinated the freelancers' tasks, a much shorter project completion deadline than the one he was actually working towards. This was the moment I realised that there were diverging understandings of collaboration at play between myself and my interlocutors. As I continued to move throughout the different teams and departments that were contributing to the client project, I encountered similar patterns of knowledge management and control. When I later traced the individual work steps along a client project, I realised that only the senior consulting team members were directly involved in meetings and client communication; this corresponded to the perceived hierarchy in the office. All other teams in the project development process were instead briefed by the previous team and were provided deadlines for completing their tasks.

The notions of collaboration in this division aligned with manoeuvring the project information (or parts of it) to achieve the goal of successful project delivery without escalation. The individual teams and their actors were not part of one organisational system, but differentiated sub-systems that selected only particular aspects of information about the project that they determined important for the receiving sub-system. This resulted in a multi-tiered working misunderstanding in the form of a "bargaining game" around delivery 
dates, as Asif called it. Chapter 9 addresses these "date games" between employees.

The working misunderstanding between myself and the employees in the organisation was, however, that my notion of collaboration assumed transparent and equal access to the project's information within the same social system. This notion was supported by the "officially communicated" idea of collaboration by the organisation's leadership team in the new joiner's training and reinforced by the open plan office set-up. However, the everyday working praxis fostered the emergence of individual teams along the project development process. Consequently, the observed communication processes showed a selection of information from the hierarchically higher team (i.e. that which was closer to the client) on what the next team should know, such as teamspecific project deadlines. However, the selection of understanding of this information by the next team sometimes differed from what the initial team expected: the next team factored in this pre-selection of information and reproduced the information within their own sub-system as "Yes, that's the date we [were] given, but they anyhow planned some buffer, so the real deadline must be later". On the practical side, this approach to collaboration led to a rather unexpected situation during fieldwork: by moving between the different teams in this multi-sited corporate setting, I frequently gained significantly more knowledge about the individual projects than the employees had themselves. This opened up a number of considerations relating to knowledge management during the fieldwork with respect to the intentional working misunderstanding.

\subsection{From a non-intentional to an intentional working misunderstanding}

Once I discovered the diverging notion of collaboration between myself and my interlocutors through the multi-sited fieldwork approach, the situation demanded that I take a decision on how I would interact with them in the context of project collaboration. If I were to openly share my knowledge about the various projects, I might bring the carefully crafted collaboration system to a point of unravelling. The alternative was to play on the ambiguities at hand, just as my interlocutors did, to keep the misunderstanding working. As outlined in the next section, I took the latter approach and shifted from a non-intentional to an intentional modus of working misunderstanding. 
As many of my interlocutors were perfectly aware of the strategic information concealment between the teams and played their role, so too did I play mine in "the other side's drama" (Reed 2006: 158) by not sharing my knowledge of the project. While the early phase of my analysis of collaboration patterns clearly involved a non-intentional working misunderstanding between myself and the interlocutors, the situation changed as soon as I gained more insight into the working practices of project collaboration that shaped each team's strategic information selection. From that point onwards, I was able to realise the ambiguities at play regarding the circulating project information and I managed my own knowledge carefully in order to tune my utterances and questions to the knowledge levels of my interlocutors. Through my actions, I actively and intentionally kept the misunderstanding working.

I did not directly voice my understanding of collaboration in contrast to the lived praxis of my interlocutors. I can only speculate on the reflections of my interlocutors on this, as I did not create a dissonance in their expected behaviour - at least, if I did, it did not become salient enough for them to raise it in discussion. It was only during the final research phase, when I followed the project execution process and frequently changed desks, that Kashish leaned over to me in a conspiratorial manner and uttered with a low voice: „I know you probably shouldn't tell me, but I saw you sitting with Neha [the project manager] this morning, so you must have talked about project GREEN. Well, at least tell me if it's on track, I fear something is boiling up there..."

Hence I was not the only person in the organisation playing this role. Kashish was also quite aware that I had understood the notion of collaboration at play in this organisation and shaped my behaviour accordingly. The way he posed his question further suggests that he was equally aware that he was violating the rules of the game by drawing on my knowledge in order to get information that had not been selected for him by the other team. Acting according to my own understanding of collaboration would have meant telling my interlocutor that I had accompanied Neha that morning during her numerous phone calls to the freelancer team leads about the status of their work. I would have also mentioned that one of them had announced the risk of a potential delay of several days, which Neha had decided not to pass on to the consulting team, as she was positive it would be fixed with a bit of overtime work. Instead, I uttered something indifferent about many projects at play right now, each with their own progress levels and challenges. It was a response that fit the working practice and, consequently, Kashish did not repeat 
his question but instead focused on an urgent client request that demanded his full attention for the rest of the day.

With my growing insight into the misunderstanding, I learned to map the boundaries of knowledge exchange and the distribution of information across the organisation. I also learned which parts of my own information about a specific project I had to "switch off" in which team, in order to not impede the set-up shaped by my interlocutor's concept of collaboration. Further, I learned when to be vague about my project knowledge when accompanying interlocutors from various teams, even though my own understanding of collaboration proposed a different behaviour. I had moved into the intentional modus of misunderstanding in order to keep the misunderstanding working.

\subsection{Working (with) a misunderstanding}

Given my pre-disposition stemming from my professional background as a Western IT specialist with a deep-rooted commitment to information provision based on egalitarian ideas of knowledge-sharing, it is not a pure coincidence that I found my understanding of collaboration mirrored in the organisation's official idea of collaboration. This congruence enabled the misunderstanding to persist unidentified over several weeks of intensive fieldwork. The working aspect of the misunderstanding lays in the fact that I was able to apply my notion of collaboration in terms of knowledge sharing for the initial phase of the fieldwork without encountering dissonance to the observed practice: I accompanied my interlocutors to briefing meetings and conference calls and eagerly absorbed their explanations of the various databases recording a project's information. With that level of insight, my idea of collaboration seemed to fit the working practices. Consequently, I collected data on how the employees in and across the various teams shared project information, and via which communication channels and functions.

Only after being in the organisation for a longer time was I able to relate an interlocutor's information on a specific project to the knowledge I gained about the very same project from another team. Gaining sufficient insight into what was communicated for project collaboration delineated my "point of unravelling" (Reed 2006) - the moment I realised the incongruence between my notion of collaboration and my interlocutors' execution of it. Until that moment, the working misunderstanding could be allocated within the quadrant typology under the locus anthropologist $\leftrightarrow$ interlocutors and the non- 
intentional modus. After the point of unravelling, however, my case study experienced a shift towards an intentional modus of working misunderstanding. In this later phase of fieldwork I consciously shaped my behaviour according to the ambiguities relating to project knowledge, in order to comply with my interlocutors' notions and practices of collaboration. Therefore, the misunderstanding can be positioned in the blank spot of the quadrant - in the category of intentional working misunderstandings between an anthropologist and interlocutors.

\subsection{Concluding remarks on collaboration as a working misunderstanding}

In brief, I can contrast my conception of collaboration as an egalitarian, openaccess approach with the understanding of my interlocutors as a strategic, fit-to-purpose concept. My conception was rooted in a view of organisations as systems that are internally differentiated by segmentation and equitable emergent sub-systems. In this view, the selection mechanisms of information are consequently less restrictive. Collaboration in my interlocutors' sense, however, was based on interaction in a hierarchically differentiated system with a functional selection of information.

In spite of these factually diverging notions, the interaction between me and my interlocutors was possible without encountering dissonance, and we were able to discuss various screens within project documentation databases and other tools used for their work. Information about the project collaboration practices at the organisation allowed me to recontextualise the situation (Gershon 2005: 103) in order to make it meaningful within the realms of my own concept of collaboration. This "parallel encoding" (Sahlins 1982) enabled an undisturbed continuity of interaction with my interlocutors due to the working misunderstanding. It remained undiscovered as long as my insight into the other system was limited to the visibility of how information was being exchanged (which matched my expectations).

This seemingly marginalised category is analytically interesting insofar as it is marked by the boundary (La Cecla 2002: 103) between the anthropologist's understanding of a concept and interlocutor's views of it. During the fieldwork described in this chapter, I was convinced of my own perception of collaboration as an egalitarian interaction. Today, more than two years after returning from the field, I am beginning to question whether my ideas of 
collaboration might constitute an idealised understanding of the term. Retrospectively, I cannot swear that my own communication strategies during my time as a project manager in the industry were significantly dissimilar to those of my interlocutors at Advice Company. I will analyse these strategies "date games" - more closely in the next chapter. 



\section{Modus intentional: Date games}

From the locus anthropologist $\leftrightarrow$ interlocutors analysis, this chapter and the following chapter (10) will shift to analyse the working misunderstandings between my interlocutors at Advice Company. In this context, I will refer to misunderstandings between the sub-systems within the organisation, as well as those between the organisational system and its environment. This chapter will illustrate the intentional modus of working misunderstandings through the collaborative practices of information control (described in Chapter 8) that I call "date games". Figure 18 positions this chapter within the analytical dimensions of the $\mathrm{L} / \mathrm{M}$ quadrant.

Figure 18: Chapter 9 on the L/M quadrant

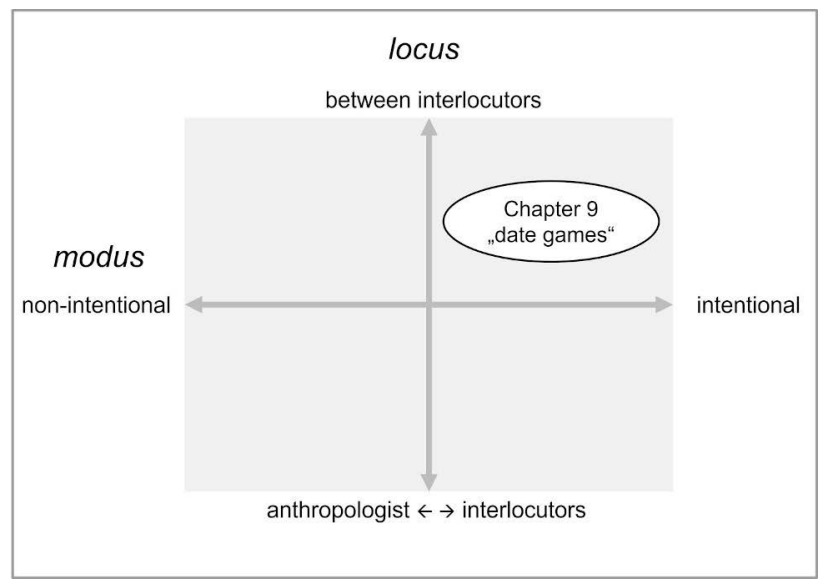

The analysis focuses around the central commodity of Advice Company the client project - and particularly the working misunderstandings around 
delivery dates. A delivery date seems a rather unambiguous, objective and clear piece of data. However, the ethnographic examples will show that the individual sub-systems strategically select information on delivery dates during the project planning phase, and these dates become intentional working misunderstandings between interlocutors.

To approach the "date games" from a theoretical perspective I will introduce the concept of double contingency through the framework of Systems Theory (Section 9.1). Section 9.2 provides ethnographic accounts of date games as working practices within Advice Company and analyses them from the theoretical perspective of double contingency in the context of working misunderstandings. The examples illustrate how the value client centricity structures the date games in the project planning stage. In Section 9.3, I contrast the use of these games in the execution phase, during which the value ground reality becomes more relevant; this reverses the direction of the games, at least to the point of escalation. Section 9.4 provides examples of date games in the system/environment interaction and traces collaboration practices beyond the organisational boundaries. I show that the date games also involve clients and highlight the circumstances under which the date games are terminated when as the working misunderstanding reaches a point of unravelling. In a concluding section, I position such cases within the L/M quadrant and reflect on the way in which delivery dates depict intentional working misunderstandings as a central element of client project collaboration.

\subsection{Double contingency and cross-system interaction}

When two social systems interact, the encounter is coined by uncertainty, as both sides know that the other may select from a range of possible actions and it is unclear which action will be selected. The other system's selection of action is unforeseeable unless a mandatory option exists. Luhmann, in line with Talcott Parsons, refers to this as contingency: "Something is contingent insofar as it is neither necessary nor impossible; it is just what it is (or was or will be), though it could also be otherwise" (1995a: 106). As the condition applies to both systems with respect to the other, it can be understood as double contingency. This leads to an interdependency whereby each system 
seeks to pre-empt the actions of the other on the basis of its own selection. This is problematic, insofar as social systems are "black boxes" to each other: ${ }^{1}$

The basic situation of double contingency is then simple: two black boxes, by whatever accident, come to have dealings with one another. Each determines its own behavior by complex self-referential operations within its own boundaries. What can be seen of each is therefore necessarily a reduction. Each assumes the same about the other. Therefore, however many efforts they exert [...], the black boxes remain opaque to one another. (Luhmann 1995a: 109)

This opacity of interacting social systems thus allows for only an assumption of the action that will be selected by the other system as a consequence of one's own system's selection of action. In contexts in which each system seeks to prompt a specific action within the other system, this is only successful on the contingency of a trigger-causality, rather than an effect-causality, unless the systems are structurally coupled. As the latter is not the case amongst the subsystems within Advice Company, their interactions remain in a situation of double contingency and are caught in a self-referential circle that is difficult to resolve: "I will do what you want if you do what I want" (Luhmann 1995a: 117).

The next sections illustrate how the interactions between Advice Company's emergent sub-systems are coined by "date games" as strategies for dealing with the other system's opacity and the double contingency situation. I will furthermore argue that intentional working misunderstandings around delivery dates provide a method of overcoming the self-referential circle.

\subsection{Date games and working misunderstandings}

The ethnographic accounts provided in this chapter pertain to cross-system communication in relation to project planning and progress tracking.

$1 \quad$ Gregory Bateson also uses the term in this sense: "It's a word that comes from engineers. When they draw a complicated diagram of a complicated machine they use a sort of shorthand. Instead of drawing all the details they put a box to stand for a whole bunch of parts and label the box with what that bunch of parts is supposed to do. [...] But it's not an explanation of how the bunch works" (Bateson 1972: 40, emphasis in original). 
They serve to illustrate how collaboration between sub-systems is organised through the use of delivery dates as working misunderstandings. Such situations do not occur with every project across the organisation, yet they are observable in various independent situations across different teams and divisions. The examples selected reflect this wide distribution of the phenomenon.

\subsubsection{Mitigating double contingency}

When a client announces interest in commissioning a project with Advice Company, the client consultants, whose function is to engage in boundary work, are the recipients of this request. As specialists in communication with the environment, they are responsible for triggering actions within the organisational system on the basis of environmental impulses. Together with communicating specifics about a project (see Chapter 10), a client sets an expected delivery date for the final project and sometimes a few milestone dates to measure interim progress. These dates are then discussed within the client consulting team and the team usually proposes that a later delivery date be agreed with the client. Client consultant Aniket remarked: „Some clients will always crunch your timelines, whatever you tell them. They push, whether feasible or not, and even if we deliver the project only 1 day before the initial deadline they say: ,See, this is why I pushed you.“

From this quote, one can already anticipate the nature of the tensionladen date game with the client systems, which are addressed in Section 9.4. At this point, I will continue to describe the process from the moment a delivery date is agreed with the client. After this point, the project is officially launched and the project development process begins, together with the mechanisms of information selection by each sub-system, as deemed necessary for collaboration (see Chapter 8). When the client consultants inform project coordinators about the project, they select the delivery date they assume is required for a successful and timely project delivery to the client. This selection occurs on the basis of assumptions about the action that will be triggered within the opaque sub-system, as client consultant Neelam indicated:

You know, the data preparation teams will be late in any case, because the execution guys will only start working when the deadline comes near... So I give the project coordinators shorter timelines, keeping the buffer for me. 
Otherwise I will be the one sitting there doing long hours to deliver to the client on time.

Along the project development process, teams are briefed by the functionally preceding team, which is hierarchically higher on the client centricity scale, and this team communicates deadlines for the tasks they are briefing. These deadlines are selected and processed within each sub-system on the basis of the same mechanism, as Asif from the project coordination team explained: „The client consultants might agree for example on 20 days [until project delivery to the client], but they give us only 15 days. And we also do the same only: when project coordination gets, say, 15th of next month to have it completed, we tell to the execution teams the 12th."

Asif describes an example for the double contingency in the cross-system interaction: When client consultants inform project coordinators about the delivery date of a new project, they select this information based on the expectation that the project coordinators will work towards that shorter deadline, so that even a delay will not result in an issue. But client consultants have no insight into the selection mechanisms within the project coordinator's system and hence cannot pre-empt the action that will be triggered within the project coordination team. Accordingly, the client consultants' sub-system is opaque to project coordinator Asif and the selection mechanisms that lie behind the delivery date are a black box for him. But equally, he makes assumptions about the alternatives that may have been chosen by the client consultants - decisions that are contingent for him. He does not know if the delivery date he was given is congruent with the client's final deadline, or if/which alterations to the date occurred to trigger a specific action within his system. He can choose to work towards the deadline given or decide that it is an artificial date that was set to allow the client consultants additional time to create their final report. Conversely, the client consultants do not know if Asif will choose to work towards the date they communicated to him or if he will select an understanding of the date as a ballpark timeline that he may or may not aim to fulfil. This is the double contingency that both sub-systems must deal with, along with all other (sub-)systems involved in the project development process.

The date games are, on the one hand, a strategy of extending the double contingency and reducing the risk emanating from the opacity of other subsystems for successful interaction. On the other hand, they enable cross-system interaction: delivery dates are bits of information that can be processed by the involved sub-systems; hence, they engender follow-up communication. 


\subsubsection{Playing on client centricity}

The double contingency lends an interesting twist to the date games, making them more than a waterfall of rational risk mitigation practices. Asif's quote suggests that the different teams suspect that the delivery date information they receive has most likely been edited (i.e. pre-selected) by the preceding team in a way that this sub-system perceives as appropriate for achieving their goal of a timely delivery. Sheeba, a project coordinator, commented upon reviewing the briefing on a new project with - as was relatively common urgent timelines: „Those consultants play oversmart on us. They have some 5 to 10 days' buffer, but don't tell us. And we only have unnecessary pressure, which we need to push down to the other teams."

Similar to her colleague Asif, Sheeba was perfectly aware of the fact that she was kept in the dark about the actual delivery dates that were agreed with the client and that they were most likely later than she was told. Project coordinator Neha explicitly mentioned the opacity of the client consultants' subsystem and the selection mechanisms that led to the delivery date she was given for completing the project: „I know the execution teams won't deliver, because they can't deliver and still I have to pressurise them. We want to understand more about the reason of pressure. Here nobody says "No" easily, first all say ,Yes'."

Apart from Neha's desire to gain more insight into the client consulting team's selection processes behind the delivery dates, her statement reproduces the internal boundaries of the organisation. As the client consultants orientate on a different value than the execution teams, who operate on the basis of the value ground reality, she expresses the notion that she has to protect the execution teams from the client-centric consulting teams (these boundary iterations are covered in detail in Chapter 10).

The quotes furthermore suggest that the project coordinators select an understanding of the delivery dates that incorporates consultants' unjustified accumulation of extra time for their final work step in the project. This leads to the interpretation of delivery dates as ambiguous and up for negotiation, rather than clearly defined and binding. Project coordinator Sandesh accordingly referred to the project planning process as a "bargaining game", referring to the delivery date "game" as a local metaphor: "You know, it's like a bargaining game, and sometimes you have to play it like that to win it."

The relative meaning of the delivery date was also confirmed by Rohan from the project execution team in the street office: „We sometimes have to 
commit to unrealistic timelines, when they tell us client needs it. And everybody knows it's unachievable, even consultants. But project goes ahead only and we deliver with delay." It might not be surprising that the rules of the "bargaining game" were determined by the value client centricity - at least most of the time. When I accompanied embedded team member Anas at the city office, he commented on his strategy of "outsourcing" a task to the standard team. The fact that he associated the term "outsourc[ing]" with the standard team, which was located within five metres of his desk, communicates the boundary between the two job types I illustrated in Chapter 6 (Section 6.1.2), which outlined the internal differentiation of the city office. Anas claimed that, in these cases, he never disclosed his real delivery date and always kept a buffer. When he needed to send something to his manager on a Friday evening, he would tell the standard team he needed it by Wednesday. When I asked for his reasons for doing so, his answer was similar to the statement of client consultant Aniket at the main office: „Because I need that buffer, as their [the embedded team's] work will most likely not be up to the mark, so I will have to spend time in reworking it. If I tell them my real delivery date the standard team might also only deliver on Friday evening and then I will be the one who is sitting late and getting under pressure." As a member of the embedded team with an overseas manager, he saw himself as occupying a more superior position on the client centricity scale than his colleagues on the standard team. This led him to feel entitled to request an early delivery date.

Similarly, project coordinator Preeti utilised an interesting argumentation strategy during an update call with a freelancer team manager at the street office. The team manager was apparently unable or unwilling to guarantee that his freelancers would deliver the outstanding tasks by the deadline they had initially agreed to. After several minutes of discussing why such a situation had occurred, Preeti raised her voice: "I can't tell the client that because of your freelancer's skill-set problem this work can't be done by end Feb!"

Her leverage of the term "client" is remarkable, as Preeti would most likely have no opportunity to tell the client anything about the project. She was not in a function assigned to boundary work. But her work location - the main office - differentiated her as an employee located in the most client-centric office in the organisation, and this suggested that she would have a closer position to the client than her colleague in the street office. She was able to 
select this message due to the opacity of the social system of the main office to the street office employee she was interacting with.

These examples show that the date games were only a superficially rational sequence of interdependent decisions, such as the kind assumed by game theory - a method of economic modelling introduced in the 1940s by John von Neumann and Oskar Morgenstern (2007[1944]). Beyond the use of date games as a risk mitigation praxis, they were also used as the basis for collaboration across sub-systems. They facilitated collaboration not only across offices, but also across teams within each office. Considering the physical proximity of each of the teams in the offices, it seems rather unlikely that such a level of information control could have been exercised. But given the internal differentiation strategies and boundary manifestations of emergent sub-systems (as illustrated in Chapter 6, Section 6.1), such a phenomenon is comprehensible.

The date games imply an idea of collaboration as a means of purposive information control, iterating the hierarchical structure of the organisation along the client centricity scale. In conjunction with the concept of collaboration discussed in Chapter 8 , the date games illustrate that information is not supposed to flow freely through the organisation for all sub-systems to dispose of. Rather, information is a resource - a commodity associated with the client centricity scale: the more directly it comes from a client the greater potential it has for manifesting the organisational hierarchy. The date games also provide insight into each sub-system's view of the other systems in its environment, with respect to anticipated actions: the decision to communicate an earlier delivery date is made on the basis of an assumption that the other system will deliver late. Such assumptions are a relevant aspect of why the delivery dates can be understood as working misunderstandings.

\subsubsection{Delivery dates as working misunderstandings}

When the client consulting teams communicate a delivery date to their project coordinators they deliberately select an earlier deadline than the one they are working towards. As a consequence, the delivery dates used for planning are not as precisely defined as the agreed calendar dates suggest. The client consultants, the project coordinators and the execution teams each assume that the communicated delivery date will not be met: "everybody knows it's unachievable" (Rohan). 
The calendar deadline that is communicated between sub-systems, which serves as a basis for interaction, is therefore a working misunderstanding: the point in time the client consultants or the project coordinators understand as the expected delivery date is not transparent to the other system(s). Within the client consulting team, the expected moment of task completion lays somewhere between the project completion date agreed with the client and the delivery date that is communicated to the project coordinators - the latter of which they do not expect to be met. When the client consultants and project coordinators interact in their project planning on the basis of the communicated delivery date of the 2oth of April, neither of the two parties encodes this date as the corresponding moment on the calendar, but as a later date. But the interaction works successfully on the basis of these calendar delivery dates.

The working misunderstanding can also be classified as intentional, because the interacting parties are fully aware of the fact that the communicated delivery date is subject to differing ascriptions. In spite of these different meanings across the systems, the communication praxis is repeated within Advice Company. Project collaboration is even dependent on the ambiguity of the delivery date, as the two sub-systems would otherwise be caught in the self-referential circle of double contingency. Hence, they need the date games and the delivery dates to operate as working misunderstandings in order to facilitate cross-system interaction.

The working misunderstanding remains at play as long as the margin between the project delivery and the client's completion date is deemed manageable by the client consultant. But at times the opacity of delivery deadlines leads to delays that endanger the timely project delivery to the client, and thus the success of Advice Company. In these moments, escalation strategies replace collaboration.

\subsection{Date games reversed: Status reports and escalation}

The rules of the game are set by the organisational structure, which provides the consulting teams with the most direct information from the client side in the environment about project delivery dates. The other teams only receive information from the preceding team in the project delivery process and must take their decisions on the basis of this information. The project planning phase is characterised by decision-making on the basis of an asymmetric 
information structure between client consultants and other teams, with interdependent selection processes connected with this information structure. For the following phase of the project execution, however, we witness an opposite information structure, as the execution teams make use of the most direct information from the other side of the organisational environment the freelancers.

\subsubsection{Flipping the game: Status reports}

When the project is in its execution phase, date games can occur in the opposite direction. At this stage, the freelancer team managers decide which information to select in the communication process - namely how long it will take for their freelancers to fully complete the given tasks and collect the data required. The sub-team at the receiving end of this communication is the project coordinator team. This team must decide which understanding to select from the information impulse at their sub-system's boundaries and how it should be processed within their own system. Such a message might include information from the freelancer team leads about a delay in the project progress; the project coordinator must subsequently decide if this delay might still be okay or if it will require intervention. The project coordinators must then take a decision on which information should be selected for the client consulting teams.

In this situation, the project coordinators exhibit information control for successful collaboration with the client consulting teams. Some use Asif's strategy, which he revealed when I accompanied him. Client consultant Raveena came to his desk and requested a status update on one of her projects that was nearing the end of the execution phase. Asif told her that he could not prepare the status update on the project that day, as they had agreed he would send her an update on Thursdays. He referred to the number of other projects he had to take care of and promised to put her project update on the top of his list for Thursday. Raveena was not content with the situation, but accepted Asif's objection. Once she left to return to her desk, Asif commented that Raveena might have escalated her requests if she had seen today's data on the project progress, which had been slower than expected. He thought that, had she seen this, she would not have listened to his explanations about the progress agreements he had made with the freelancer team leads for the outstanding work. He expected the progress to increase significantly over the next few days, and he hoped to provide Raveena with an update on Thursday 
projecting a delay that was minor enough to remain within her tolerance margins and to skirt escalation.

In this case, Asif selected information about the project status according to his understanding of the agreed delivery date. The next example shows how project coordinator Neha received pre-selected information from the execution teams on project GREEN ${ }^{2}$, which was almost a week past-due. Over lunch, she complained with the other project coordinators that she was in trouble because the freelance team lead had apparently given her "fake information”, as she called it: „Last week he told me that the work tasks are completed and the freelancers would only have to consolidate their stuff. But now he tells me that they need time until Sunday to complete the work." She said it was not clear to her if he had intentionally given her the wrong information or if he had just blindly trusted his freelancers' statements about having the work tasks completed and passed this update on to her without verifying it. Her colleague Preeti confirmed that notion:

It's a trust issue. He might well have given you wrong info and now blames it on the freelancers. That's very easy, because they are not part of the organisation, nobody will ask them, nor will they have to face any consequences. Same is with the client consultants. They never mark [copy] us on client emails to not share the timelines they have agreed with the client. Sometimes they push us to complete the work tasks even one, two months before the project delivery presentation is due.

Preeti's comment illustrates her suspicion that the client consulting teams use their functionally more direct access to the client as an information advantage to play date games. But her comment also illustrates that the freelance team lead might use his information advantage to play the date game in the other direction, by selecting information according to his system's understanding of the actual delivery date. As he has access to the most direct information on the work completion status, he is in a position to select which information is communicated to his project coordinator colleagues in order to prevent an escalation - just as Asif did with his client consultant colleague.

The date games operate according to the value client centricity, with subsystems that more closely interact with the client most knowledgeable of the agreed project completion date with the client. Consequently, the client consultants are able to select information for communication with the next sub- 
system, the project coordinators. The employees at the street office work at the least client-centric location in the city and hence receive the most indirect and pre-selected delivery dates for the completion of work tasks. During the execution phase, however, the information levels get flipped upside down. The work tasks providing the data basis for a client project are fulfilled by the freelancers. The freelancer team leads operate according to the ground reality as the leading value, and during the execution phase, these roles have the most direct information on the project status. The freelancer team leads select the status update information they perceive as relevant for successful interaction in order to prevent escalations of issues to management. In this phase of the project, ground reality is the more relevant value (Figure 19).

Figure 19: Project Phase and Information levels

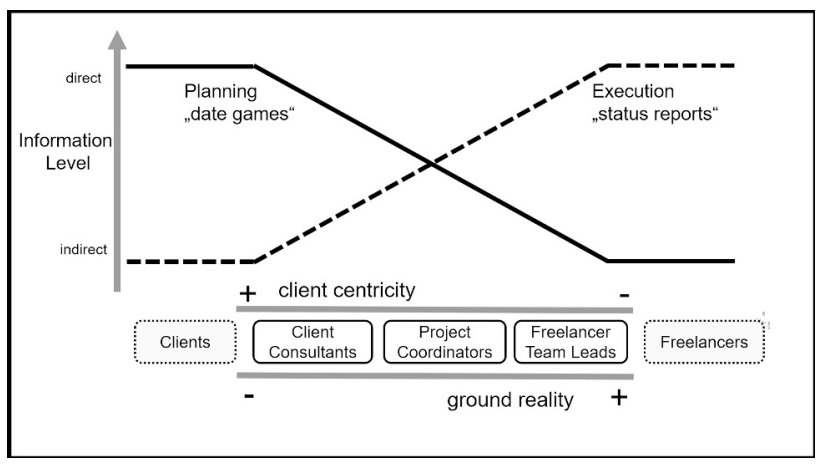

Figure 19 illustrates furthermore that project coordinators are in the middle, between the client consultants and the freelancer team leads, and must consequently master the different information selection processes at play. In order to execute their function, they must operate intentional working misunderstandings to maintain interactions without provoking dissonance. Project coordinator Neha's manager referred to this as "diplomacy":

Neha: I had a feedback call with my manager yesterday. I was told I have to be more diplomatic. How can I do that?

FM: What do you mean by diplomatic? 
Neha: My manager said I should be more diplomatic in my communication to not have issues coming up, no escalations to him, you know. But it is very difficult for me. I am used to be[ing] very open and say[ing] how it is [...] So here it is very difficult for me to not tell things or sometimes I feel I almost lie about it. But my manager said I should be more diplomatic when things go wrong and not tell everything at that moment. I was told I have to manage things more on my own now without the help from the management level and be more diplomatic so that things don't get escalated, I should be more independent. I have to mentor these two new joinees, so I should be like that.

"Diplomacy", as a trait that Neha's manager demanded that she develop, can hence be regarded as the ability to manoeuvre successfully between different understandings of delivery dates and status information. For the project coordinators, it refers to the art of manipulating the working misunderstanding in relation to a delivery date on both sides, interpreting both the delivery date they have been given by the client consultants and the actual deadline by which they need to complete the tasks. They must also be able to use a delivery date to collaborate with execution team leads, in order to enable them to complete the project tasks in time for the client consultants, despite their differing interpretations of that deadline.

\subsubsection{Escalations as emergency breaks}

While the level of information is inverted from the planning phase to the execution phase, the overall hierarchical differentiation of the organisation along the client centricity scale remains. As a consequence, the working misunderstanding about status reports during the execution phase can be instantly brought to a point of unravelling through the mechanism of escalation. When a client consultant sees the successful and timely delivery of the project to the client jeopardised, even when his or her own buffer is factored in, he or she must inform the next management level of the situation.

Once a project status is escalated to management by a client consultant, the collaboration between sub-systems immediately ends. Until that moment, the interaction between sub-teams is a carefully balanced communication structure based on intentional working misunderstandings that cater for the opacities of the sub-systems. When a project is raised to escalation, however, the client consulting team - or its manager - can request direct and detailed 
information on the execution process and insight into the mechanisms of the other, less sub-systems. An escalation of a project status to higher management attention is followed by a wave of teleconferences and meetings, during which managers of the involved teams review the situation in detail and have their staff work on micro-level action plans. The choice of an escalation path to enforce project completion is regarded as ultima ratio, as explained by client consultant Gopal:

There are two options how you can make people work for your project quicker and harder. The primary one is rapport. If you share a good rapport with people they will work for your project quicker when you ask them to. If you don't, then your work might be put back and might get lapsed. So this works based on the relationships we have. The second option is escalation via the managers. But via this route a person might do the work maybe twice or thrice for you, but then it will not work anymore, so I try to avoid that if possible. Issue is, when you escalate, people don't work only. So what happened now on this project was, we figured we have to work hard on the execution tasks. So I in a first step I involved Srinat [a colleague with a good informal connection to the execution teams], but the people just did not move at all. Then we played it hard via email escalations to both division heads. This helped to get a clearer picture of the situation and all that is still to be done. But basically, now we have to plan in next week to pacify their [the freelancer team leads] burned egos and be friendly. And only then they will start working. You have to treat them with a hot/cold blow strategy.

Through Gopal's perception of having "burned egos" from an escalation, one can assess its dysfunctional nature in the organisational system. Using escalation to cut the interaction across sub-systems (and hence the working practice of date games and status reports) might achieve a short-term goal, but it has serious consequences for the communication system. The working misunderstandings with respect to delivery dates and status reports are important for collaboration across the organisational system, and escalation strategies cannot serve as an alternative strategy to overcome the double contingency. 


\subsection{Date games across system boundaries, and their limits}

Thus far, I have shown how working misunderstandings around the delivery date play an important role for the successful collaboration of the organisational sub-systems when delivering a client project. When these date games are played within Advice Company, the framework is set by the organisational structure of information directness and sub-systems' hierarchical positions along the client centricity scale. When timelines for project delivery are negotiated with clients, however, the communication process sprawls across system boundaries. In this section, I will illustrate this with two case studies that show how the date games are played beyond organisational limits and sometimes reach a point of unravelling.

\subsubsection{Boundary work positions}

Within the organisation, client consultants process the delivery date information for further handling. But these delivery dates are the outcomes of the consultant team's "boundary work" (Holtgrewe 2003: 64). The consultant teams comprise a functional sub-system concerned with the organisational boundaries with the environment - in this case, the clients. As I illustrated in Chapter 4 (Section 4.2), the freelance team leads perform the same "boundary work", but they do so in order to craft and maintain the organisational boundaries with the freelancers (Holtgrewe 2003: 65). While both sub-systems are structurally similar, their disparity in status is determined by the organisation's dominant value client centricity that is of fundamental significance to the client projects.

Client consulting teams are in a hierarchically higher position than the other sub-systems that perform "boundary work", such as the freelance team leads. Client consultants paradoxically are, however, in a weaker position to shape the relationship with the environment according to the organisation's own interests, as the decision to place a project order with Advice Company is taken solely by clients. In contrast, the decision to hire freelancers for their services lays with the freelance team leads; similarly, the HR department determines the type of relationship the organisation has with tertiary education 
institutions ${ }^{3}$. Hence, in these cases, the decision-making lies within the organisation.

The delivery date games that are played across organisational boundaries represent an interesting analytical category due to their close connection in time. Organisations differentiate themselves from their environment not only by communicating their boundaries, but also through time dimensions. Luhmann speaks of the emergence of system-immanent time, which must still adhere to the time of the environment (Luhmann 1995a: 185). By analysing the manipulation of delivery dates, one can trace how the organisation processes impulses from the environment - from clients into its own context - and how varying time dimensions play a role in the organisational boundaries. We have seen above that the date games build on and (re)produce internal differentiation. Similar system-environment date games are at play with clients, yet with distinct differences to the internal date games, as I will illustrate in the following two cases from two different client consulting teams within Advice Company.

\subsubsection{Playing client delivery date games}

Sneha, a client consultant, informed Deepak, a junior colleague, about a client request that had just arrived in her inbox. After exchanging general information about the project's topic and scope and a preliminary action plan, Sneha asked Deepak to call the client to speak about timelines (i.e. delivery dates). During the phone call, the client told Deepak that she wanted the project to be delivered by the 15th of December. Deepak promised to check if that would be possible, but he warned her that it might not be achievable. As his reason, he explained that the earliest the project execution teams could complete their tasks would be the end of November, depending on the freelancers' productivity.

But instead of revising her deadline, the client argued that she needed the project report and recommendation for a planned top-level management board meeting on the 17th of December. She would need the recommendations to form the basis of a discussion at that meeting, so she could push for

The HR department decides whether or not to establish a campus recruitment agreement with a business school and the execution team leads are responsible for the decision to utilise a freelancer's service on the basis of Advice Company's skillset requirements. 
a strategic decision before the year's end. Deepak again announced that he would check on the feasibility of her request and get back to her. He got up and walked to Sneha's desk to discuss this phone conversation with the client. With a frown, Sneha said that she would not buy into the client's delivery date timelines, and her idea of a realistic project delivery was "something like midJanuary". Then she told Deepak in an explicit, though not unfriendly tone that he should not share with clients any information about when the project execution work would be complete. The reason for this is that the client might push for delivery of the report soon after the execution team's work tasks were complete.

While both looked at the calendar on Sneha's computer screen, on which she clicked back and forth between the weeks, the two began to discuss whether the client's board meeting on the 17th of December was a bluff. Each brought to the discussion facts they knew about the client's organisation (relating to its management structure and their experiences with past projects). They finally concluded that the client must have mentioned the meeting as a bluff, and that the said board meeting would not actually happen - at least not on the 17th of December. Therefore, Sneha calculated the project plan with a potential delivery date of the end of December. Before concluding the ad-hoc meeting, she reiterated to Deepak that he should not share timelines about individual work tasks with clients.

The next day, when I asked Deepak about the timelines for the new potential project, he told me that he had decided not to send the revised delivery date proposal to the client, but to ask (via email) for a phone call to discuss the timelines. When I asked if there was a chance for alignment he replied in the affirmative, because Sneha had said that she would be able to deliver the final project in January, but would have the option to offer a partial update and preliminary analysis on the 2oth of December. He rated this scenario as a good basis for agreement, as both he and Sneha had come to the conclusion that the client was bluffing about the board meeting on the 17th of December. Deepak therefore felt confident that the client was creating a buffer for herself that they would be able to negotiate.

The call with the client was scheduled for the afternoon. When Deepak and Sneha gathered in a meeting booth to prepare for it, Sneha proposed to enter the discussion with a project delivery date of the 17th of January, with a preliminary report a week earlier, on the 1oth of January. Her final comment before Deepak started to dial into the conference call crisply summarised her bargaining strategy: "The client will be upset with this, but we anyhow should 
put this timing into our initial proposal. Why crunch timelines at the beginning, let her beg for it."

Consequently, the client got an initial delivery date proposal of the 17th of January, which was then "negotiated and mutually agreed" for the 3rd of January with a preliminary report on the 2oth of December.

This example illustrates the strategies employed by the consulting teams in executing their "boundary work". Both Advice Company and the client organisation had the target of finalising the project in the most convenient timeframe for each of them and the double contingency made this a challenge for both sides, as such targets were rarely in congruence. The client opened the communication by selecting a first delivery date, which Deepak and Sneha the boundary specialists - processed within the system as "unachievable".

The follow-up communication Deepak selected was aligned with the project process within the organisational system. His feedback was that the client's requested deadline was "unachievable because execution teams only ready too short beforehand". The selection of understanding from the client system can only be inferred from the follow-up communication, for which the date of the executive board meeting was selected to iterate the validity of the expected delivery date. Hence, the selected understanding of Deepak's response to her request was definitely not "unachievable", but might have been "if they stretch they maybe can".

Processing this selection of understanding in the client's system hence led to the decision to "increase pressure" by selecting an utterance that rebuked the client's internal hierarchical structure (executive board), its system-immanent time (meeting date) and the constitutive necessity of decision-making in the system's time context (requiring a strategic decision before year-end). The selection of understanding from both Deepak and Sneha of this utterance is remarkable: they selected to understand it as a bluff - determining that the strategy employed internally at Advice Company for collaboration across subsystems was being used by the client to achieve a buffer on her end.

Both boundary specialists assumed that the decision of the client system was to select an artificial meeting date in order to increase the pressure on them; hence, they decided not to align their delivery dates. The client, respectively, was most likely aware that her counterparts at Advice Company would not understand her delivery date request as the date that would ultimately be demanded. Therefore, "delivery dates" became an intentional working misunderstanding in this cross-system communication: both sides were aware 
of the other party's differing understanding, yet this misunderstanding was used to foster the project initiation process.

According to Luhmann's concept of communication, information cannot flow directly to another system; instead, it causes an irritation at the boundary that gets processed according to the system's structural framework. The communication described above illustrates that social systems do not communicate directly, but via the selection of an utterance and understanding. When Deepak said "unachievable", the client responded with a revised delivery date request. If there had been direct communication between the systems, then this follow-up communication would not have made sense, as the status "unachievable" would have triggered follow-up communication to inquire about a possible delivery date.

It becomes clear how these intentional misunderstandings of the delivery date are used for the interaction: they reiterate each system's structures and shape them through internal processing mechanisms. Sneha emphasised the organisational border of Advice Company when she said - twice - that it was not advisable to give a client information about the planned delivery of tasks from the execution teams, as doing so would provide too much information about the organisation's decision-making options when playing the date game with the client. This informational edge with respect to the internal processes of the organisation maintained the equilibrium between clients and consulting teams in the delivery date games, as clients had an advantage, by default, through their decision-making power.

\subsubsection{When the date games stop working}

The second example, from a different client consulting team, illustrates what can happen if this equilibrium is hampered by an advantage of information and decision-making power on the client's side:

Manhas and Brijesh were client consultants on the same team. During a cigarette break, Manhas explained to me that they were "arming" themselves for a discussion about the delivery dates and pricing of a new project opportunity with a - in his words - "stressy client". The client had basically demanded that Advice Company deliver the project as soon as possible and had given the consultants his expectation of what he thought was an achievable delivery date. Back at the desk area, Brijesh and Manhas prepared for the call. Both reviewed the background information about the project before calling their teammate at Advice Company's Delhi office. All three discussed the 
delivery dates they would suggest and the arguments they would use to back up their proposal, as they liked to avoid committing to very tight timelines.

The twist that added complexity to the upcoming call, as Brijesh explained to me, was that the client was a former employee of Advice Company who had "moved to the client side" almost two years ago. Therefore, he knew the organisation's internal processes around client projects, including, of course, the date games. Consequently, his knowledge about the organisation, in combination with his decision-making power with respect to assigning projects to the organisation, gave him a favourable position, as - to him - Advice Company was not completely opaque and contingent. A few minutes prior to 3.00pm I accompanied Brijesh and Manhas to a meeting room. Manhas dialled into the teleconference and the three of us sat around the phone in the middle of the small table, which was set to speaker mode.

As foreseen by Brijesh, the phone call proved difficult. The client gave the two consultants a "hard time pressurising on delivery dates"4 ${ }^{4}$ In contrast to the client from the previous example, who referred to system-internal dependencies on management meetings or other relevant deadlines (e.g. year end) in her own organisation, the client here built his argumentation solely on the project process within Advice Company. He demanded to see the internal project design for his review in order to give his input to it. He had also prepared his own calculation of the project's time plan, with an assumption of how long the execution teams should take to complete their tasks and when the project could be finalised by the consultants. When both Manhas and Brijesh tried to push back on these delivery dates, he continued with his argumentation that they had support from the teams in the city office and consequently should be able to deliver. The gap between the delivery dates the two parties aimed at amounted to several weeks, not days.

During the phone call, the tone became more aggravated with each negotiation round. When Manhas told the client that Advice Company would not be able to count on support from the city office team, the client continued to remind his counterparts of his professional past at the organisation. He was surprised to hear about the situation and concluded that something must be wrong with the company's strategy. When he worked at Advice Company he had support available and was of course also expected to increase his workload to achieve timelines for client projects. 
The discussion continued for another 25 minutes, during which Manhas, whom I had come to know as a calm and quiet person, started to show signs of impatience and frustration: between the client's wordy argumentations he would grimace and/or push the "speaker mute" button on the phone, so the three of us could hear what the client was saying but any conversation on our end would not be transmitted to the client. This function enabled Manhas and Brijesh to enter into lively discussions amongst themselves, during which both heavily gesticulated.

But it didn't help. The final agreement was that the two consultants would revise their timeline to bring it closer to the client's expectations. The client did not budge from his initial delivery date. When the call ended and Manhas finally disconnected the phone, he commented that "this guy is just talking on and on, lets nobody speak". While we left the meeting room and walked back to the desk area, both Manhas and Brijesh continued to discuss the call. Upon sinking into his office chair, Manhas uttered with a frowning side glance towards me that now his work intensity levels had doubled ${ }^{5}$.

The communication in this example is remarkably different to that of the previous example. Here, the delivery date game was played from a special point of departure: the client had previously worked at Advice Company and therefore had once been part of the organisational system he was now interacting with from his new position at a client's organisation in Advice Company's environment. He had selected an understanding of the initial delivery date Manhas gave him according to the interests of the client system he was now a member of (i.e. to achieve as early a delivery as possible). But his information processing not only occurred along the guiding difference of the client's system, but it also incorporated his knowledge of Advice Company's structure, or what he believed to be the structure. This can be inferred from his follow-up-communication: he did not move from his initial proposal and justified his iteration through his assumptions about the client project development process at Advice Company.

The client's decision to insist on his initial delivery date was grounded on the certainty of his knowledge of the possible decision options of his counterparts at Advice Company and which option they might choose. Thus, the 
interaction was not determined by double contingency. Manhas tried to balance the asymmetry in negotiating power by selecting an utterance about the unavailability of the support team at the city office, attempting to indicate to the client that his assumptions about the client project process within Advice Company might no longer be valid. This follow-up communication allowed for the assumption that the client might have selected an accurate understanding of the information. But the client made clear - through his reference to client centricity as the leading paradigm within Advice Company - that he still had knowledge about other aspects of the organisation's structure that he could consider for his decisions in the delivery date game.

An expression of this notion of intrusion into their system could be registered in Manhas' reaction of switching off the phone microphone to discuss with Brijesh a possible resolution to their situation. He tried to actively reestablish the boundary towards the client by technically cutting the communication channel. At the same time, he sought to reinforce the social system of him and Brijesh, through conversation. The call nevertheless ended with an unravelled working misunderstanding and two slightly dejected consultants. This situation can be compared to Bohannan's ascertainment that the colonial working misunderstanding only remained "working" as long as the two systems were kept apart (1964:25). The date games as working misunderstandings could not be played with this client, as the ex-employee was aware of the processing mechanisms within Advice Company. Apparently, this was not a singular phenomenon. Cathy, a client consultant I did not accompany but happened to share good rapport with, replied to my question of whether she had ever had former Advice Company employees as clients:

Yes, that happens indeed from time to time, as quite a few colleagues are happy to move to the client side. And this is always potentially problematic. Why? Because they believe to know exactly what is going on here, but they don't understand that their knowledge is several years old. And since they left, a lot might have changed. I once had a client who accused me of having made a mistake and complained with my manager. But in the end it came out that here simply our processes have changed and we didn't do the things anymore the way he thought we would.

I described the above meeting incident, which I had experienced several months before our conversation, and asked if she could relate to it: 
Oh yes... [laughs] I can very well relate to that! See, on the one hand, yes, that client was right insofar as it is theoretically possible to deliver the project in a shorter timeframe. But this only works if we consider only that one client's project. In reality we need several projects running in parallel to cover our business, and so all are working on several projects in parallel. Plus, if something goes wrong on our end and we have to do re-work, then the shorter timeframes are again unfeasible. So, netnet [the strict net] timeframe of a project might be shorter, but not in actual practice. Of course it is difficult to deal in a project timing conversation with a client, who actually knows all this. Because he is also very aware that you just can't say officially: "Yes the actual sum of work steps is shorter and we could deliver your project earlier, but we have to consider other clients' projects and to cater for potential issues on our side." This is why it is always a bit tricky to negotiate with excolleagues on the other end at a client.

Other ethnographic studies have described this "breaking point" of the misunderstanding (Losonczy and Mesturini Cappo 2014:2), as well as a "cognitive unravelling" (Reed 2006: 159) or "fallen mask" (Cole 2014: 545).

I have demonstrated in these cases how date games as intentional working misunderstandings around delivery dates are a constitutive element of crosssystem communication that enable each system's boundary work. However, the second case study illustrated the result of the opacity of the two interacting systems ceasing to subsist. Similar to the escalation strategies in the previous section, this situation ultimately prevents the misunderstandings from "working".

\subsection{Concluding remarks on intentional working misunderstandings}

The date games, as played both inside the organisation and across the organisational boundaries, use delivery dates as an intentional working misunderstanding. The different (sub-)systems attach their own meaning to the dates through system-specific selection processes in the communication events; this enables an uninterrupted series of follow-up communication and decisions - constitutive operations of the autopoiesis of the organisational system. 
In the communication processes observed in the example of the internal team-specific project deadlines, the hierarchically higher team selects information that they assume will produce their desired outcome once processed by the subsequent team in the process chain. The selection of understanding within this next team and the processing within the sub-system, however, might be different from what the previous team expects. I have illustrated how the subsequent team factors an assumed pre-selection of information by the preceding team into its decision. The fact that all interacting parties are aware of the differing ascriptions of the communicated delivery date makes it an intentional working misunderstanding.

During the planning (i.e. date games) phase of a project, information levels align with the organisational structure along the client centricity scale. However, once the project is in the project execution (i.e. status reports) phase, the situation reverses: in this phase, the freelance team leads have the most direct up-to-date information on the project's progress and the orientation changes towards ground reality as the more relevant value.

This assumption of a pre-selection of information and an intentional working misunderstanding relating to the meaning of "on track for delivery date" was reflected when Kashish asked me to reveal knowledge about the execution progress of his project GREEN, as he feared "something might be boiling up there" (see Chapter 8, Section 8.2). He was aware of the selection processes that had occurred before the project's status update had arrived with his team, and that his idea of "on track" might subsequently differ significantly from that of the other sub-systems. This incongruence between information power and hierarchy level during the execution phase strongly characterises the second phase of the client project, which depends on the intentional working misunderstanding around communicated delivery dates for bottom-up collaboration.

In most cases, the top-down planning phase did not unravel the misunderstanding, as to the system's structure prevented individual sub-systems from gaining insight into the other sub-systems' available information (e.g. the definite agreed delivery date with the client). But the situation differed in the bottom-up situation. If project status information that had been transmitted to the consulting teams failed to be understood in line with the interpretation of the delivery date they had initially selected in the planning phase, an escalation process would start. Therefore, I argue for the inclusion of another aspect of working misunderstandings in the toolbox of analytical 
categories: the distinction between ongoing working misunderstandings and those that terminate at a point of unravelling.

Through the involvement of consulting team leads, instant direct access to project status information from execution teams could be obtained. At this moment, the collaboration would end to make room for a more intense and resource-binding mode of communication. To this point, however, collaboration would rely on intentional working misunderstandings of delivery dates (and status updates) to enable successful interaction between the sub-systems, as project coordinator Sandesh concluded in an interview:

We [project coordinators] feel that the consultants know it [the delivery date] is unachievable, we know it is not do-able, and the execution teams definitely also know it. Yet we go ahead only - it's "I-pretend-I-don't-know" style of working here that does the job.

This quote connects the working misunderstanding around date games to the intentional modus: all parties were aware that the communicated delivery date had been pre-selected by the previous sub-system and they therefore allowed themselves to attach their own meaning to that date. The impulses that arrived at the sub-system's boundaries in the form of information about a delivery date were not congruent to the selection of the sub-system's understanding of it. Because of these ambiguities at play around the actual delivery date, which all interacting systems were aware of, the project collaboration led - in the vast majority of cases - to the successful, timely project delivery to the client. This also underlines the assertion of organisational sociologist Nils Brunsson that "a lack of rationality is not necessarily a disadvantage for organisations" (2006: 35). While this chapter has illustrated how the intentional modus of working misunderstandings can serve as a fruitful analytical category, the following chapter will trace the non-intentional modus of working misunderstandings. 



\section{Modus Non-Intentional: Project Representations}

This chapter seeks to open the "black box" of the client project and illustrate its different representations along the development process. I will show that each representation depicts a non-intentional working misunderstanding between the Advice Company sub-systems (Figure 20). For this, I will go beyond the project planning layer that was the focus of Chapter 9, in which I demonstrated how collaboration between sub-systems on a client project functions on the basis of intentional working misunderstandings around delivery dates.

Figure 20: Chapter 10 position on the L/M quadrant

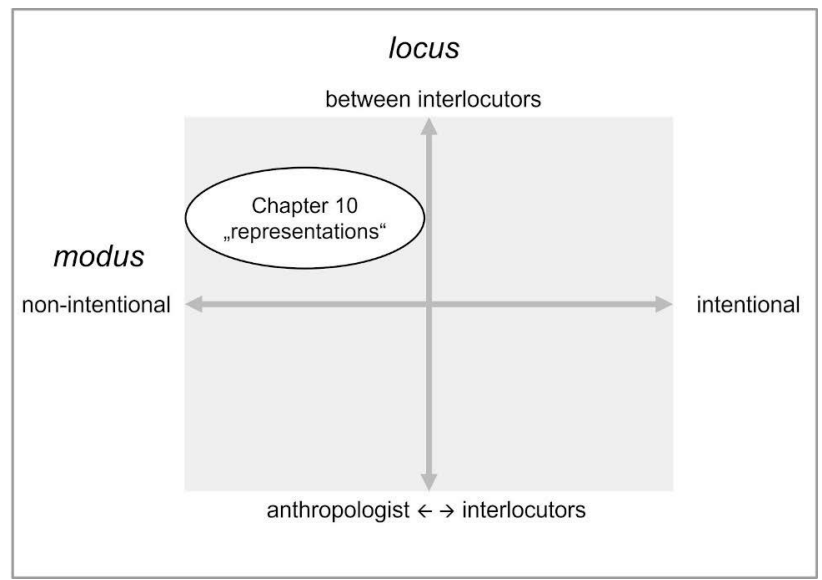




\subsection{Organisational decision-making and "black boxes"}

When a client files a project with Advice Company, he or she aims to get advice on a strategic question such as whether to invest resources in a certain business area. As I outlined at the beginning of Part I, decision-making is one of the constitutive operations of an organisational system. The advice provided by Advice Company supports a client's decision-making process when decision options are connected to a high level of uncertainty. As the "institutionalised purpose of decisions is to achieve certainty, to determine and stabilise the future" (Brunsson 2006: 7), decisions must be taken for a client's organisation to continue to exist. Information stimuli from a system in the client's organisational environment are relevant for the client's internal decision-making process (Luhmann 2006a: 340f.). Once a decision is made, the uncertainty under which the choice was made no longer plays a role for subsequent decisions made by the organisation. The circumstances of the previous decision-making situation - including whether there were alternative attractive choices, whether the options had clear pro or contra arguments or whether opting for one choice over another was merely based on chance and accompanied with doubts - is no longer visible. This reduces complexity, and such reduction is one of the key interests of system emergence, as all decisions are made on the basis of prior decisions; in other words, decisions engender decisions (Luhmann 1997: 830).

When a decision becomes fact, its production circumstances are sealed in a "black box" - a term stemming from cybernetics, where it stands for a complex array of commands or machine processes about which no details need to be known except for the inputs given and the outputs received (Latour 1987: 2). Luhmann refers to the black box to illustrate the predicament of double contingency in cross-system interaction (1995a: 109). Latour employs the idea of black boxes in the sense of Gregory Bateson (1972: 40) and describes the process of "black-boxing" as changing the status of a scientific proposition from its initial, fragile phase - during which it stands under debate and faces controversial challenges - to a broadly accepted concept - a scientific fact or an assumption upon which subsequent research builds on, rather than challenges (Latour 1987: 7).

The projects filed by Advice Company's clients can be compared to such black boxes. The client knows the input (strategic question and briefing) and is presented with the output in the form of a report and/or presentation. Information about the project development and the steps taken towards the 
final recommendation is provided to the client in the form of an official standard project process and quality control documentation. What really happens before the project is finally delivered, however, is kept mostly within the organisational boundaries, subject to rigid information control (i.e. selection) by the boundary work specialists - the client consultants. Latour's concept of black boxing (in terms of knowledge production) and Luhmann's understanding of the enclosed uncertainty of a decision-making situation once it has been taken, provide links between the seemingly incompatible positions of Systems Theory and Actor Network Theory (ANT).

\subsubsection{Opening the black box}

The theoretical basis for unpacking the black box is based on concepts from ANT - namely "circulating references" (Latour 2000: 88) and "translation chains" (Rottenburg 2009 [2002]: xxxii referring to Latour 1987) in knowledge production. Rooted in the realms of science and technology studies, ANT seeks to uncover the conditions of science in the making and emergence of outputs (scientific facts). The key assumption of ANT is that a wider understanding of agency encompasses not only human beings, but also nonhuman entities (Latour 1996: 369). Whether human or not, an actor in ANT is able to actively or indirectly affect a network. One of the most prominent examples of this is Michel Callon's study of commercial scallop cultivation, in which he uses the same vocabulary for all actors, irrespective of whether they are fishermen, scientists or scallops - a principle he calls generalised symmetry (1986: 200). These basic assumptions inhibit a direct connection of ANT with Systems Theory: Luhmann does not account for individuals, be they human or non-human, as acting entities of a social system. To Luhmann, communication is what constitutes a social system and reaffirms its boundaries, not a group of individuals or actions (Luhmann 1986: 175). Nevertheless I would argue that it is indeed possible to utilise these two concepts of circulating reference and translation chains from ANT for the system-orientated analysis of client projects in this chapter. In the following, I will explain these concepts and describe how they can be fruitfully used in conjunction with Systems Theory - to trace a client project through the organisational sub-systems of Advice Company. 


\subsubsection{Translation chains and organisational systems}

The concepts of translation chains and organisational systems can be approached through a case study from Latour, in which he shadows a research project seeking scientific answers about the soil quality at the boundary of the Amazonian rain forest (2000: 36). Latour accompanies the project from the initial extraction of soil samples to the submission of the scientific publication to a journal. The case study illustrates that the initial referent - a piece of land in the Amazonian region - is followed by representations of it in the course of the research process: a modicum of soil in a cardboard box, a code number for its colour and a graph printed on paper in the final publication. All of these representations, however, refer back to this piece of soil - the initial referent. Through processes of amplification and reduction, representation of an initial situation or object (i.e. referent) evolves and is translated into the specific format that is able to be processed by the environment it is meant to interact with.

It is important that all communications refer back to the initial referent which is the proposition of the circulating reference (ibid.: 89). Yet because it is necessary for the information to be understandable to each receiving system, it must be reduced and/or amplified in different ways - or, in Richard Rottenburg's, terms, translated:

Because this individual pieces are not direct substitutes for an external reality but instead bring forth a cascade of further substitutes, one is never dealing with a single referent but rather with a diversity of internal or transversal referents that have been organised into a chain such that they support themselves as they proceed along it. From this perspective a representation is always a cascade of re-re...representations. Because the practice of representation is best understood as a translation, I will call this a translation chain. (Rottenburg 2009 [2002]: xxxii , emphasis in original)

The idea of translation chains shows a parallel with Systems Theory insofar as the different sub-systems in the organisation are acknowledged to select and process information according to their function in the project development process and the organisation's guiding difference. Although Luhmann strongly opposes the notion of the "transmission" of information (Luhmann 1995a: 139), he sees ritualisation as the function of "translat[ing] system-external uncertainties" into internal schemes, and as an organisation system's choice to internally thematise uncertainty using self-created protocols and 
other documents for evidence (ibid.: 185). As I demonstrated in Chapter 9, such processes of information selection are at play at Advice Company with respect to delivery dates, both for system-environment communication and communication between sub-systems. In order to grasp the processes behind these different representations of a client project, I will adopt the concept of translation chains and apply this to the project development process at Advice Company. A similar analysis of translation chains is documented by Kiepenheuer-Drechsler in an ethnographic study tracing the projects of integration policy-makers in Germany (2013: 247) and by Rottenburg in the example of a fictitious development aid project that draws closely on his experiences of various projects he accompanied in the 1990s (Rottenburg 2009 [2002]).

As I demonstrated in Chapter 8 through the concept of double contingency, collaboration between departments and across organisational boundaries relies on the selection of information and understanding within each sub-system. The argumentation in Chapter 9 centred on intentional information selection based on assumptions of how this information would be processed in the receiving sub-system. In this chapter, the viewpoint will expand to examine the flow of client projects along the organisational value chain, from a project order to its delivery. I will show how the client project becomes a chain of translations and how it circulates through the organisation, with each sub-system creating its own representation of the project. Each of these representations refers back to the initial referent of the project order, yet presents it in a form that the respective sub-system can process. I will further argue that these representations are unintentional working misunderstandings that not only enable, but also structure the collaboration process. I will consider the way in which processes of information selection, utterance and selection of understanding are at play in client projects.

According to Systems Theory, a project cannot be considered an acting entity and neither can the employees of Advice Company be considered as individuals in the analytical focus. Rather, it is these employees' communication that constitutes the organisation's social system. Such communication is shaped by selection processes, which align with the employees' functions within their respective organisational sub-systems. A project cannot move through the organisation independently, and nor can information flow from one sub-system to the other. It is only communication about a client project that stimulates the next sub-system based on a selection of understanding for processing according to the receiving sub-system's framework. 
I will trace and connect these impulses of information selection and utterance in one system and their stimulation of another system to Latour/Rottenburg's translation chain model: impulse and stimulus are both references to the same client project, yet are never congruent as they undergo frequent selection processes of reduction and amplification. Alexa Färber incisively asserts in her study of knowledge representation at the Expo that shares of reality are not always congruent to their corresponding representations (2006: 15). I will show, in the next pages, how these different and incongruent references are exactly what constitute working misunderstandings around a client project.

\subsubsection{From client centricity to ground reality, and back again}

In Chapter 7 (Section 7.3), I illustrated the basic flow of actions associated with a client project, from winning the initial bid to delivering the final report to the client. I connected the project process to the organisation's guiding difference client centricity/ground reality to illustrate how a project's "strategic" tasks are connected to the value client centricity and how its operational tasks are orientated along ground reality. Chapter 9 illustrated how these different values employ intentional working misunderstandings for (mostly) successful interaction during the project planning and status update phases. The remainder of this chapter will show that the entire project development process is textured by the guiding difference client centricity/ground reality and denotes the black box of organisational functioning I seek to open. Taking the client project as the central commodity, I will trace its representations along the individual sub-systems and offices and demonstrate its relevance as a working misunderstanding. The following sub-sections of this chapter address the key steps along the project development process and the analysis commences at the beginning of the process - at the organisational boundary between Advice Company and the client systems in its environment.

\subsection{Lead management: Translating uncertainty}

Client projects are the main exchange commodity that generates income for Advice Company. In contrast to other basic resources that are drawn from the environment (e.g. skilled graduates), financial income must be immediately and perpetually generated in order for the organisation to maintain its 
existence. Advice Company's services are also necessary for maintaining its clients' organisations by supporting their decision-making processes.

Figure 21: Allocation of Section 10.2. in the project process

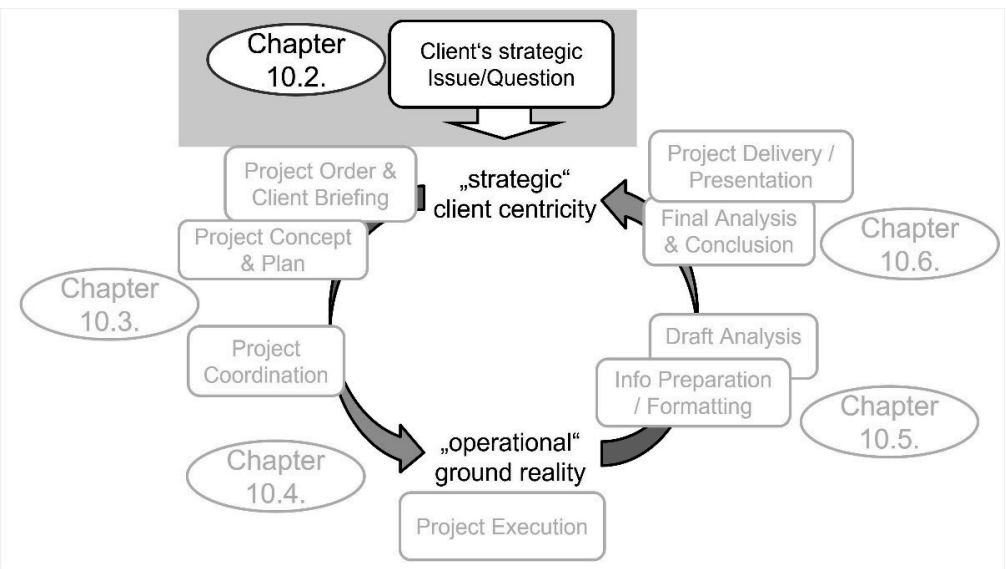

\subsubsection{The forecast meeting}

Before a project can begin, a client must agree to give the project order to Advice Company. While the organisations in Advice Company's environment rely on decision-making for their existence and draw on the services of Advice Company to support their decision-making, only a handful of projects are agreed upon upfront as repeat contracts. Hence, Advice Company's income from client projects is predominantly irregular, and there is a degree of uncertainty with respect to the organisation's survival. Unless an organisation has a monopoly on the service it provides, clients will always have the choice of hiring a competing organisation. Even when contracts are initially secured, in the event of financial shortfalls at a client's organisation, they might be subsequently cancelled.

According to Luhmann (1995a: 185), an organisation translates this contingency into internal processes and documentation. In Advice Company, this translation is conducted by a top-down allocation of sales targets to management-level consultants and through regular meetings to monitor the status of leads (project opportunities) in order to foresee potential income resource 
threats as early as possible. I attended such a meeting with the first client consulting team I accompanied. All seven team members who were present in the office at that time joined the meeting, and two others dialled in via telephone - one from the office in Delhi and one from home. When all computer and phone connections were established, the manager ${ }^{1}$ who was leading this client consulting team started teasing Aniket, who had dialled in from home and whose connection was mediated by the black, star-shaped speaker phone in the middle of the table. The manager asked if he was sitting comfortably in his home at the window, with his feet up on the table with a chai tea and samosas in front of him. A wave of laughter flooded the room and the meeting began with an announcement of the agenda. This agenda centred on an spreadsheet file, which Bright had opened on his laptop; as his laptop was connected to a projector, all who were present in the room could view the file. Aniket and his colleague in Delhi were also able to see Bright's screen through the meeting software they were logged in to.

Along the spreadsheet's $\mathrm{x}$-axis were months, and the $\mathrm{y}$-axis featured the names of client organisations. For the future months, potential projects were listed - some with a concrete project name, others with a topic area and still others with only a question mark. But all of these leads had an estimated budget associated with them, which was discussed in terms of its likelihood of actualisation. Each row (i.e. project) was addressed and the team member in charge of the client gave a brief description of the project's background. Meeting participants commented on the leads' feasibility of becoming real project orders during the time span indicated. In the course of the meeting, the numbers revealed a potential gap in the revenue target for the next quarter. As a mitigation strategy, the manager asked Bright to send a new project proposal to the potential clients; Neelam was asked to check on the status of the most recent project offers sent to clients; and Sunidhi, one of the new joiners, was asked to contact some of the clients on the list that had a less clear prospect of generating further income.

After the discussion and review of future projects, the next item on the agenda was reviewing the status of ongoing and completed projects. These projects were monitored in the same spreadsheet, but in a different section. The focus of the discussion around ongoing projects was their status on the project development process and potential issues that might obstruct an here. 
on-time delivery. The main concern with respect to completed projects was whether the final payment had been received. Meeting participants were assigned to check with the finance department or the client for outstanding payments. This task was very unpopular amongst the consultants and sometimes even led to conflict. At a coffee break, Ruchika recalled a situation in which a client had disrespectfully addressed one of her consulting team colleagues as a "money collector" in an email response, after the young colleague had reminded the client about an outstanding payment. Ruchika said about that incident:

When I saw that mail I was thinking: oh just somebody, either one of our managers write an email to the guy that yes, money collection is also part of her job, but also many other highly valuable tasks. She is the most junior in the team and has been working nightshifts the last two days to get a report document to the client, and she just happened to ask about the invoice in the same email while sending the deliverable through. The report document she sent had been requested very urgently, and our manager had called us every few minutes when it will be finished and he put a lot of pressure on us, we could hardly work. So how does she feel in that moment?! The problem is that you never know if and when the managers will stand up for you, actually there is nobody standing up for you in that particular matter.

Ruchika's quote provides insight into the team's internal hierarchies and frictions, but it predominantly shows the perceived misfit of "valuable" (i.e. client-centric) tasks that the client consultants did not perceive as meaningful boundary work.

When the meeting was over, almost two hours later, the data file containing all of the projects showed a few updated cells with more details about potential new projects. For other potential projects, the status field displayed only a question mark. Months before a client project became a reality, it would exist in a cell on a spreadsheet and would gradually take shape, from a vague question mark to a brief reference of the topic and, finally, to a concrete project name. The question mark might not even indicate a project interest announced by the client; it could merely indicate a potential opportunity that had been identified or assumed by one of the team members about what might be saleable as a project to the client, despite the opacity of client systems for Advice Company. 


\subsubsection{A structure of uncertainty}

The above example illustrates how the perceived uncertainty and serendipity of the project business in Advice Company's environment is translated within the organisational system into the format of a spreadsheet file - the epitome of structured data. Spreadsheets are the main tool used in finance and accounting departments to track an organisation's financial status. Furthermore, such files are generally used to manage and aggregate quantitative facts and data. Considering this connotation of a spreadsheet, the immense disparity between the uncertain prospect of client projects and their precise representation in a spreadsheet becomes salient.

Furthermore, spreadsheets have the technical capability to easily aggregate figures from individual teams and departments to produce a financial business forecast for review at the board level. Such figures reflect Latour's idea of a black box through the translation mechanisms described by Luhmann: uncertainty in Advice Company's environment is met with Advice Company's expectations of potential project orders, which are translated into spreadsheets. These spreadsheets are produced by each team and aggregated into a forecast of future revenue. On this basis, decisions are made by management seniors.

But it is not only the specific media into which the environmental uncertainty is translated, but also the arrangement of data in the file that illustrates the structural change from the amorphous cloud of unsecured project possibilities in the organisation's environment to a format that can be processed internally. The more or less informal phone conversations with clients about upcoming developments in their organisation and important changes in the national economy - as well as client consultants' expectations of getting clients' approval for new projects - find their representation in a matrix of data on a spreadsheet. This is analogous to the mechanism Latour observed in his case study of the "pedocomparator" - a tool used by soil research specialists to pack soil samples into standardised, small carton boxes that are subsequently placed in an assigned compartment in a grid arrangement (2000: 61). Similarly, the potential projects in Advice Company's environment are "packed" into cells on a spreadsheet in a grid set-up that corresponds with the organisational structure.

Furthermore, updating project opportunities from unnamed potentials to actual sources of income retraces the gradual solidification of a lead with amorphous texture to a concrete client contract. This suggests a quasi-evolu- 
tionary development of project opportunities once they find their representation in a spreadsheet cell. However, such a development does not always occur: As I passed by client consultant Gopal's desk on my way to the coffee machine, he stopped me with a "Morning, got a minute? Things don't go as planned". I asked him how the preparation for an important client meeting (taking place the next day) was progressing. He told me that it had actually been cancelled by the client and pushed back four weeks. He assumed also this new date to be rather volatile and I thought that the change might have actually granted him a few relaxed workdays. So I asked him why he still seemed to be concerned. He explained that the delay would cause a severe budget gap in the second quarter that had not been calculated for and this would cause an issue for his budget forecast. In this case, the project's question mark status did not transform into a real project at the right time, despite assumptions that it would.

In significant contrast to Latour's example from the Amazonian rain forest, a client project finds its representation in the organisation even before it is a concrete project moving along the development process. Here, it is Luhmann who accounts for these translation processes from an organisation's insecure and contingent perception of the environment to concrete information that it can process. This is also an example of Luhmann's structural coupling (Luhmann 1991: 1432): as soon as the project prospect becomes real, it takes on a structure - a space in which it can fit further processing. In this respect, I would argue that Luhmann, despite the incompatibility he claims between the suppositions of Systems Theory and ANT, not only agrees with the translation chain model, but even amplifies its scope to incorporate the stage of uncertainty about the environment of a social system.

\subsection{From strategy to project actions}

The previous section outlined how the amorphous stage of a project opportunity transforms into solid income for the organisation during the forecasting cycle. Section 10.3 focuses on the project development process from the point that a client files a project with Advice Company. 
Figure 22: Section 10.3 in the project process

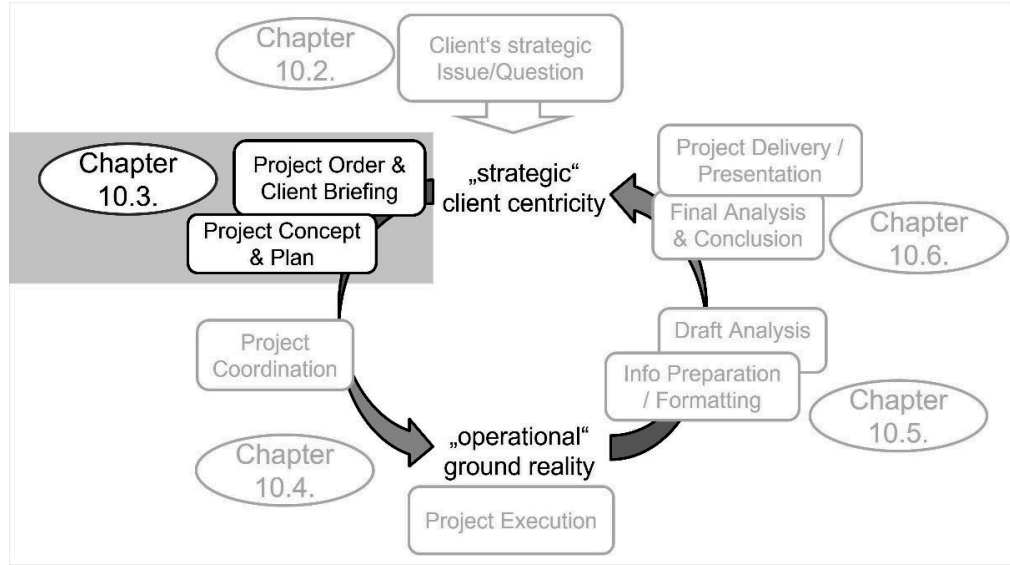

\subsubsection{The project briefing as a communication process}

A project is "communicated into being" (Sprenger 2017) through an official briefing. Depending on the client organisation and its relationship with Advice Company, this briefing might take the form of an email with a spreadsheet in a pre-agreed format (comparable to an order form), a face-to-face meeting or a conference call; whichever form it takes, the briefing outlines the requirements of the project in detail and often supports these notes with presentation slides. This step depicts the communication process that marks the beginning of the project. The project-constituting communication process consists of information that is selected by the client and its transmission through verbal utterances and/or documentation.

Clients' selections of information can differ as widely as their transmission formats. Several clients - mainly the largest ones - have specialised organisational roles assigned to performing the boundary work between the organisation and Advice Company. While the persons in these roles serve as the primary contacts for administrative questions, their primary task is to translate their system-internal need for decision-making into information that can be processed by Advice Company. They are knowledgeable of the services provided by Advice Company and understand the relevant differences in scope, price range, delivery timeframes and types of input needed to constitute a suf- 
ficient project briefing for each service. These boundary work specialists, who perform a significant amount of the translation activity between the systems, optimise the information selection and utterance for system compatibility. Here, the systems are - again - structurally coupled. But this is not always the case, as Cathy (client consultant) explained:

This [project briefing] can vary from client and division. Major clients sometimes have a kind of form, mostly a spreadsheet, in which they write very specific details about what they want from us. At that level it is almost like an order. But obviously, this [is] only possible, when you have knowledgeable people at the client side, who transform what is needed into Advice Company's language, you know, like using exactly our terms for the different things. And they also speak of themselves as those interfaces, who organise the successful collaboration work. That's the easiest. Then there are clients, who have a few presentation slides about what they expect, which we can discuss. Sometimes, our contact person is a manager, who then speaks a lot about the bigger strategic intent and other things, which are not really relevant, until we reach their concrete issue. And then you might talk to a client, who has been told by his boss to book a project with us for the go/no-go decision at their end. These people then have no idea what kind of services we offer. And this means you have to work out with them together at the meeting how a project might look like.

In the latter case, the translation process is performed through the selection of understanding of a communication made by Advice Company's client consultants. Their function is to translate the client's need for decision-making into the service offering of Advice Company that best corresponds to the client's need. This step delineates the first node in the translation chain: The client's need for decision-making finds its representation in the project briefing for Advice Company and its subsequent translation into the organisation's service structure, pertaining to project types, deliverables and timeframes. Depending on the type of briefing, the information undergoes selection mechanisms that vary from complete pre-alignment to the terminology and service structure of Advice Company to an unstructured summary of ideas in the client's system "language". At the latter extreme, the translation effort lays predominantly with Advice Company's client consulting teams, who must select an understanding of information. Advice Company's selection of understanding translates the client's need for decision-making into a form that is compat- 
ible with the organisational structure - aligned with its teams, process and timeframes - and thus makes the information usable.

\subsubsection{Project briefing and working misunderstandings}

Despite being boundary work experts, client consulting team members see the potential for misunderstanding in this first translation step, as the following situation shows: I was having a morning coffee chat in the cafeteria with Sakshi, a client consultant, when Ruchika walked past and spontaneously joined us. Ruchika spoke about her current nightshift work on a project for which a client wanted a last-minute decision to support. Ruchika's team had conducted the first phase of the project in three days and would have to run through the entire process at similar speed. With a sideways smile to me she said I should have been on the late evening phone calls with the client, because: "The potentials for misunderstanding and the likelihood for something to go awfully wrong shoots up a lot in such crunched timelines, as we might not get exactly what the client is actually asking for." Delighted about the way in which the conversation was going, I asked what she would do if she felt the misunderstanding might rise. Her answer was to have everything documented, in email. "This", she said with a frown, "doesn't really prevent the misunderstanding, but at least we have a chance to prove how it came about in case it really becomes a real issue."

I asked if she had ever encountered such a "real issue" - a situation in which the misunderstanding had become apparent. Indeed, she recalled such a project, which had taken place several years ago, when it was only during the final delivery presentation to the client when the client said the report was not at all what had been asked for. Ruchika painted the picture of a client that had gone through an internal restructure during the project phase and consequently changed the stakeholders involved. Therefore, she concluded, the information on what had been initially defined as a requirement "got lost between the various handovers". But she described the situation to have been a real disaster, and claimed that such a thing had only happened to her once during her almost 10 years in the business. She said that minor versions of this scenario tended to occur frequently, "especially when things are getting a bit hectic". But from her view, major situations only tended to occur when there were too many stakeholders on the client's side and when one department on the client's side did not understand how their requirements might differ from what another department might need. 
Ruchika's explanations of the pitfalls for misunderstanding in the briefing phase illustrate two points: First, she affirms that for the "disaster" she mentioned, the project and its intended scope became a working misunderstanding between the client and Advice Company that was maintained throughout the entire process, despite several interim updates to the client. In this situation, the working misunderstanding reached the "point of unravelling" (Reed 2006) during the final delivery presentation. Second, she holds that minor working misunderstandings frequently occur in situations under time pressure, yet these do not tend to lead to a point of unravelling or a "disaster" as extreme as the example she gave. In her view, working misunderstandings seem rather to be concomitants of high-performance project work. Nevertheless, she was aware of them and consequently documented Advice Company's selections of understandings in her emails to the client. She employed this risk mitigation strategy in order to be equipped for the eventual situation that a working misunderstanding might not work anymore.

Furthermore, she refers to instances of different understandings of the project across the client's various sub-systems; in such instances, Advice Company's representations might not refer to each of these systems' presumptions about the project. From this we can infer that she views the project as a working misunderstanding within the client's organisation: although the various sub-systems in the client's organisation might refer to the same project, they might have substantially differing understandings of what the project is actually about.

\subsubsection{Internal briefing and mechanisms of reduction}

When I accompanied Karan, a project coordinator, he mentioned just after lunch how happy he was with how the day had been going and that he would likely complete his to do list that afternoon. Only minutes later, in what felt like an ironic comedy scene - a colleague from the client consulting team appeared and announced that he had just received information from a client about a bid win for a project. The project order would be signed by the client soon and the work would need to begin sooner rather than later. Could Karan join the project briefing at $2.30 \mathrm{pm}$, in 20 minutes?

Karan and his manager Duniya joined the client consultant Faisal for the briefing session. As the meeting had been spontaneously arranged, no meeting room was available and we instead gathered around a table in one of the semi-open chat corners. Faisal opened his laptop and showed a few slides of 
a presentation that bore the client company's logo and had obviously been taken from the client's original presentation (the slides numbers were not in sequence and the word "cont." appeared twice in a heading, indicating several slides should have been available on the subject, though no related slides were shown). Faisal went through the slides, recalling what the client had said with respect to certain points. After spending about five minutes on three slides about the project background, the next 20 minutes of the meeting centred on the retrospective time planning of the activities that would be needed to reach the delivery date that Faisal had indicated to Karan and Duniya. The briefing ended after 35 minutes and Karan was tasked with confirming the budget figure and timeline by the end of the day.

Back at his desk, Karan wrote the new project's name, "RUBIN"2, on a fresh sheet of his note pad and, below it, several lines of actions and a rough estimate of the work efforts associated with each of them. Then he opened several chat windows in parallel and made phone calls to discuss with his colleagues from other departments whether his assumptions might be right. These conversations focused solely on the work tasks. The actual topic and decision-making need of the client did not play a role - they were subsumed under "this new RUBIN project". It was not a surprise that Karan's day did not end at the casually early time he had envisaged after the lunch break. When we met again the next morning he was still chasing his colleagues for pricing confirmation. In addition to writing actions on his note pad, Karan had printed his standard "to do" list for a project, to which he had added the project name RUBIN to the top right corner and the name of the client consultant. The roughly 25 checklist points were grouped into the different project phases he would have to manage from that point onwards until the work tasks were complete and the client consultants produced their final report. He explained the phases and checkpoints to me while opening the official forms in an online tracking tool associated with each phase.

The representation of project RUBIN was, by this point, a printed, standardised to do list individualised with the project name, the responsible client consultant and the deadlines associated with each action. This was an example of the break between form and materiality in Latour's translation chain model (2000: 85) - in this case through the mechanisms of reduction: Faisal selected the information on project RUBIN according to what his client consulting team would require to process it. For utterance, he selected a briefing session 
with a presentation as an impulse that would be received by the project coordination team. Likewise, Duniya and Karan selected the understanding of the project briefing according to their sub-system's framework, and the stimulus from the intra-organisational environment resulted in a momentum within their own team. This momentum was translated into the various follow-up communications that Karan began. The representation of project RUBIN as a sequence of action steps on Karan's standard to do list, which constituted an information format that could be processed by his sub-system, can be contrasted to the format of Faisal's briefing.

This situation provides insight into the various nodes in the chain of representations: as the project moved to the next sub-system in the project development process its materiality transformed into a reduced set of actions to be managed. While each action step referred to project RUBIN and the client's need for Advice Company's support in a decision-making process, the actions were information that could be processed by the relevant sub-system of project coordinators, or at least what the client consulting sub-system selected as relevant information for the sub-system.

The following case illustrates the information selection and reduction processes at play from another angle: Project coordinators Mayank and Asif, who sat close to each other, were each attending two different teleconferences when client consultant Aniket appeared at their desk area. He first approached Mayank to talk with him about a new project. After a few sentences, Mayank shrugged his shoulders and pointed with one hand to the telephone, indicating that he was in a call and could not attend to Aniket's request at that moment. Aniket then turned around to Asif, who switched his headset to mute mode but left one side of the speakers against his ear so he could continue to listen to the conversation in the call. With a move of his hand to this ear, he showed that he could not fully devote his attention to his client consulting colleague.

Although Aniket must have been aware that Asif was also attending a teleconference, he started to talk about a project that urgently needed to be pushed forward. Asif frowned and pointed out that this was, in his opinion, not only tight but even unrealistic, and that Aniket would never manage to get all of the required information clarified with the client in order to start the activities needed from all groups. But Aniket insisted that the briefing to the execution teams at the street office should happen the next day and suggested that he could start with just a small sub-segment of the tasks and continue when all was planned out in more detail. He said that he would see the project 
with "you" (the project coordinators) from now onwards and that he had only come by at this moment "to make you guys aware".

Asif lifted his hand, indicating that Aniket should hold his conversation for a second. He pushed the "un-mute" button of his phone and actively participated in the teleconference by announcing his support for the suggestion made in the call. When he muted the microphone once more, Aniket offered to sit with Asif to briefly review the project. Asif said he could do so at 5.00pm, but Aniket pressed his lips together and offered 6.30 to 7.00pm; Asif replied that he would have left the office by this time. Aniket pretended to be surprised, but Asif and Aniket had been working together for almost two years and both were completely aware of each other's usual working hours. Nonetheless, Aniket asked why he would not be there, and while returning to his desk, uttered that official office hours were until 6.30pm. In the hour following the conversation, both agreed via chat to "sit together" the next morning at 8.30am.

This example illustrates the nature of the break between the client's strategic need for decision-making and the project's representation as an executable entity. The information Aniket selected about the project presented it as a set of work activities that the project coordinators had to fit into their sub-system. The boundary between these systems was not only perceived logically by Aniket, but was also depicted territorially within the office, and Aniket actively crossed this territorial boundary in an effort to transmit the information. When Asif indicated with his follow-up communication that he would not be willing to assign the new project in the timeframe suggested, Aniket reminded Asif of the official office hours in the wider organisational system and their obligations in connection with organisational membership.

Aniket's communications oriented on the value client centricity and leveraged his higher status as a client consultant on the client centricity scale (as did those of Faisal, the client consultant in the previous case). Asif countered Aniket's demand with the argument that the client and/or Aniket would not be able to provide all of the information he needed for the execution teams so quickly. Asif hence communicated according to the value the ground reality in this case as a counter-balance to client centricity. Asif challenged Aniket's client project request, an action clearly opposing client centricity. In this case, the ground reality value appeared as a relativisation of client centricity, or a demarcation of boundaries for the opaque meaning of client centricity. 


\subsubsection{Project representations as unintentional misunderstandings}

Hitesh from the project coordination team perceived the diverging project representations of the client consultants and his team as reflecting a need for "more effective alignment". He described that the client consultants took a "download" from the client and gave a "download" to the project coordination teams; this metaphor emphasises the hierarchy of the client centricity scale even more explicitly than "briefing". In contrast to Asif's ground reality-orientated response, Hitesh argued in accordance to the client-centric value: in his opinion, there needed to be more time invested in the "download" step, and he wanted his consulting colleagues to "share experience from the client and to get us more involved". To my surprise, his teammate Asif voiced a similar viewpoint just a few days after the incident with Aniket:

We want to have more involvement on the consulting side; we need to understand what the client wants. I mean, we have to build up trust by being clear what the client wants. See, when I say to the service people here, that I want a tea, I might get a nice, fresh leaf tea or a simple one from the machine [instant powder tea produced by the coffee machines in the office]. It is like the Chinese whisper, you know.

Asif argues here for a more client-centric alignment of the project coordinators. I will come back to this seemingly paradoxical reference to the two opposing values in the next section and at the end of this book. For the time being, I would like to concentrate on the qualitative difference of these cases to the date games described in Chapter 9. The descriptions here suggest the absence of intention in the incongruence of the information presented about a project in the transmission process. Hitesh described the two-step process of "downloads" with the implicit assumption that representations would be more congruent if more time were spent on them. He did not verbalise that an intentional selection of a reduced share of project information arrived at the boundaries of his sub-system, but he clearly perceived his understanding of the project as different to that of the client consultants.

With his reference to Chinese whispers, Asif expressed a similar notion of unintentionally reduced and/or altered information on a client project, yet positioned it in the realms of misunderstanding through his example of the different products associated with "tea". But in contrast to the tea scenario, in which both interacting parties very quickly realised their differing associations of "tea" as soon as the cup stood in front of them, a client project's diver- 
gent understandings were likely to remain undetected, beyond the subtle notion that only a brief reference to the project would be processed by the project coordinators' sub-system. Project RUBIN and its multiple counterparts found its representation in a reduced share of action steps that formed the basis for a project understanding. This represented a "re-re-representation" in the sense of Rottenburg (2009 [2002]: xxxii) of the client's strategic question as initial referent voiced in the client briefing. Such representations did not substitute the external stimulus of the client; rather, they were a series of connected representations that were employed and understood at various aspects of the translation chain and project development process within the organisation.

\subsection{The client project as a plan and the "ground reality"}

The previous section illustrated how the client project becomes a representation of the client's need to make decisions. From the external impulse in the form of the project briefing, the boundary work experts select an understanding that subsequently serves as the basis of further selection within Advice Company. The client project, as the central commodity, can therefore be understood as an unintentional working misunderstanding, as the parallel encoding of the project meaning is variously perceived by the employees as a consequence of the multi-level "download" procedures that are conducted by the individual sub-systems. While the translation chain - to this point encompasses nodes within the main office, Section 10.4 puts the spotlight on the node between the project coordinators at the main office and the freelancer team leads at the street office. This node is located at the boundary of two hierarchically distinct sub-systems at two office locations on opposite ends of the client centricity scale. As the street office operates on the basis of ground reality as a value, successful interaction is even more challenging than the interaction in previous translation nodes.

\subsubsection{Briefing calls: Perspectives from two office locations}

As described in Section 10.3.3, once the project coordinators receive the "download" from the consulting teams and the deadline for the project execution phase, the client project is represented as a list of tasks for the execution teams to complete. These tasks are furthermore detailed in yet another spreadsheet file that bears the name of the project and the different 
Figure 23: Allocation of Section 10.4 in the project process

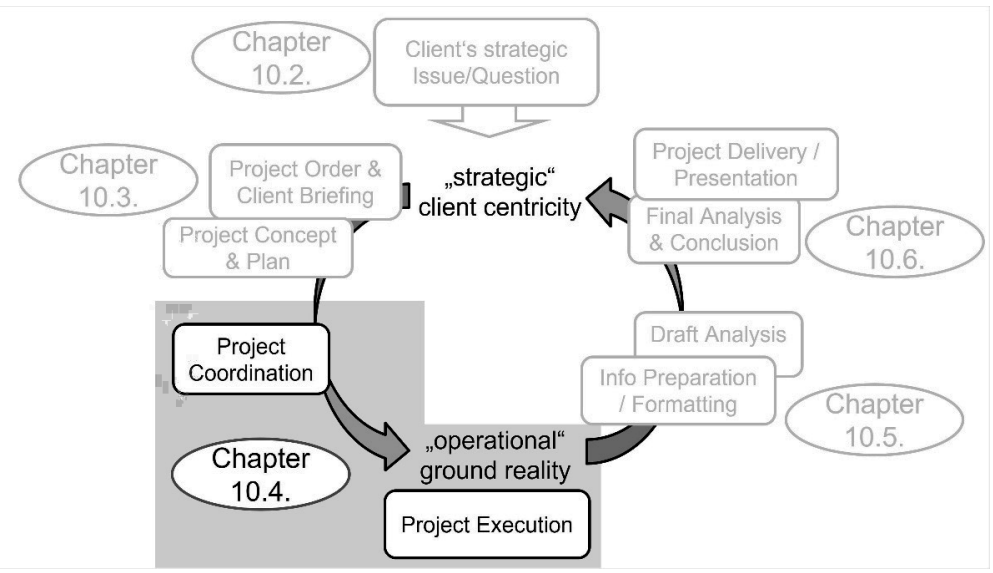

teams across India who will be involved in completing the data collection activities. ${ }^{3}$ For each team, the assigned work packets in different categories, such as business areas, are listed below. Thus, a client's recommended strategic decision is represented in a spreadsheet that is, again, aligned with Advice Company's organisational structure of office locations and teams. Both the information that is selected by the project coordinators and the format chosen for communication constitute the initial communication with the next sub-system - the execution teams (or, more precisely, the employees who coordinate the work of freelancers). This interaction is organised in the form of another briefing session, which almost always takes the form of a teleconference, as the execution team leads are based at various street offices across India ${ }^{4}$. Depending on the project, less than a handful of different street offices might be involved in smaller projects, while major projects can require a dozen or more locations to contribute to the project with their freelancers' work. The call is typically scheduled a few days beforehand via email, through which the dial-in details and (ideally) the briefing document

To protect Advice Company's identity, this analysis makes only generic reference to "(data collection) activities", "actions" and "tasks" (used synonymously).

4 For reasons of anonymity I am not able to disclose the total number of street offices run by Advice Company. 
and description of the work tasks are also shared. I attended several briefing calls, at both the main office and the street office, and it is from these calls that I collected the following cases. The first briefing call is described from the perspective of employees at the main office:

When I was sitting with the organising project coordinator in one of the meeting rooms of the air conditioned main office with a coffee in front of me and the speaker phone in the middle of the table, the boundary with the other organisational sub-system became almost materially salient. To organise the briefing call that I was sitting in on, Asif had spent a good 30 minutes in the past two hours ensuring that the participants would actually join. He had contacted each of the five execution team leads individually via mobile and landline phones to remind them of the briefing call at $3.00 \mathrm{pm}$. He had also sent out email invitations two days before and monitored the responses. In two cases, he had convinced colleagues to join despite them claiming to have conflicting events scheduled.

Asif sat at the meeting room table and dialled into the conference. One participant was already there, and Asif started a casual conversation with him. In the following minutes, three of the other four participants joined in, as announced by the characteristic "beep" of the phone software. Asif checked the names of the attendees on his list and sent a text message to the missing participant's mobile. Then he sent a chat message to Kanika, the client consultant for the project, to tell her that the briefing was starting. He officially opened the call by asking if all of the participants had the briefing documents printed and in front of them. All of them assured him that this was the case. Two of the four participants had dialled into the call from locations outside the street office: one seemed to be at a public café or plaza and another was attending the teleconference whilst travelling in a rickshaw. From time to time we heard the all-present horn sounds or the colleague giving directions to the rickshaw driver. Asif did not comment on this situation, but only asked the colleague after a few minutes to "mute" her phone unless she had any questions to ask. The background sounds were amplified by voices that flashed up from time to time, which I later identified as stemming from another briefing call that was taking place within the office for a different project.

Client consultant Kanika entered silently through the glass sliding door of the meeting room. She took a seat and concentrated on her mobile phone, apparently occupied with another issue she needed to deal with. Asif and Kanika had previously agreed that Asif would conduct the main parts of the briefing and Kanika would join in if needed. Asif went through the briefing file line 
by line and asked for feedback on whether his words had been understood, demanding an explicit "Yes, that's clear" or "No questions on that" from all of the four execution team colleagues. After 15 minutes, the mobile phone connection of the colleague travelling in the rickshaw weakened significantly and we heard the automatic voice of her network provider say that "The conversation partner is temporarily unavailable" for 20 seconds, until the line was finally cut off.

Once Asif could be heard again by all participants, he continued with the briefing. All of the tasks seemed to be very clearly conveyed until he reached the assumed productivity rate of the freelancers for the work packages. Kanika had assumed twice of what the execution team leads felt was realistic, and the discussion became so intense that Kanika decided to actively enter the conversation in order to defend her assumptions. One of the team leads emphasised their current difficulties in finding suitable freelancers and at the same time accused "you head office people" of "play[ing] down the reality". But Asif sharply terminated the argument by announcing that he would be calling everybody individually after the conference call to discuss their respective views and to "work out something".

He later told me about the motivation for his intervention: that it would be "easier to convince them of the plan at hand when talking to them one by one", as "in a conference situation it could be a discussion forever". With this intervention and mitigation offer from Asif, the briefing finished after 40 minutes. Kanika and Asif remained in the meeting room for another 10 minutes to review the timelines Kanika wanted for the project. After a bit of negotiation in light of the controversial discussion with the execution team leads, they agreed on a deadline a full week later than Kanika's requested date. Kanika confirmed this with a "Don't be late!"

As an example of a briefing call from the perspective of Advice Company's street office, the following situation occurred: When I accompanied Sachin at the street office, he attended a briefing call for a project managed by Sharan, a project coordinator I had accompanied just two weeks prior in the main office. I sat in a row of three people in the bigger office room of the street office and the phone - shared between their desks - was moved as close to Sachin's work area as the cord allowed. The phone was switched to speaker mode at maximum volume, and so were another two phones at a distance of five and eight metres, respectively, which were both connected to the teleconference line. At first I thought Sachin might have forgotten about this briefing call, as 
at 4.00pm he was standing next to his manager's desk, pointing at the laptop screen and lively discussing an email relating to a project issue.

But then he suddenly checked his mobile, walked to his desk and bent over his computer screen to bring up the email about the briefing call. He opened the attachments and sent them to the printer while dialling in to the conference. He fetched the briefing documents from the printer at the end of the desk row, adjusted his chair and looked at the printed pages while completing the dial-in procedure. By $4.10 \mathrm{pm}$, all of the attendees had connected to the conference call.

Sharan, the project coordinator at the main office, conducted the briefing. It was strange for me to hear his familiar voice through the speakers and I automatically pictured him sitting in the meeting room with the glass door. While this scenery unfolded in my mind's eye, I realised that I hadn't imagined my current setting in the street office when attending previous briefings at the main office. Despite the background noise of the office, the other teleconference attendees' environments and a constant cracking on the line, Sachin was attentive and asked plenty of clarifying questions about the briefing documentation.

I found it fascinating that he could remain concentrated for almost an hour, while I struggled to understand Sharan's words amidst the cacophony of sounds. My head seemed close to exploding upon reaching its capacity limits to handle the manifold impressions, sounds and stimuli. The communication felt more direct and "real" in comparison to the communication I had experienced at the other two offices: it seemed as if this location was missing the filter of "corporateness" that the employees had referred to in their comparisons of the three offices' atmospheres. Suddenly, the meaning of "ground reality" seemed to materialise and the distance to the client-centric parts of the organisation seemed to reinforce the contrast of atmospheres at each end of the telephone line.

Later in the call, Sachin was in doubt over whether the assumed handling time for each work package was realistic. He thought that the efforts might take much longer than anticipated by the project design, and that this would have a direct impact on cost. He put the speaker on mute and discussed this with the colleague next to him, who told him with intense gesticulation to point it out right away. But Sachin said to me in a whisper that he would bring it up at the end of the call. Indeed, he raised this question at the very end. Sharan reacted as indifferently as Asif had by saying "Yes, yes, potentially this might be the case in a few categories", and mumbling something incom- 
prehensible about the need to catch up on it separately. Then Sharan asked if there were any more questions and ended the call immediately, referencing another meeting he had to prepare for. Later in the afternoon, Sachin told his colleague about Sharan's reaction and the rushed ending of the call. When I had an opportunity to talk with Sachin about this during a cigarette break, he voiced his opinion that Sharan had been completely aware of the issue, but did not want to discuss it over the call. He decided not to send an official email but intended to "maybe catch him via phone [in the] next days..."

On the one hand, these examples illustrate how interactions were practised between the project coordination and execution teams. The selection of information and understanding spanned the main office and the street office. This interaction was characterised by the hierarchical disparity between the more client-centric main office (with the project coordinators) and the most distant locality to it: the street office (with the execution team). The briefing call had been initiated by the project coordinators, who hosted the virtual space in the form of the teleconference into which participants from the street office dialled in with passcodes that had been sent to them beforehand. Asif was also aware that he was dealing with a sub-system that was not structured similarly to the main office. He therefore called participants beforehand to make sure they would join the briefing and did not choose to perform other actions that were more relevant to them - more relevant according to the ground reality's value. The project coordinators also provided the project information and thus the content of what would be discussed. The briefing call was led by the project coordinators and the focus seemed to be on sending information rather than receiving feedback. Both Asif and Sharan ended the call quickly when critical remarks came up. Their roles were hierarchically higher and they had the power to end the call at their discretion. This fits with the understanding of briefing calls as "downloads": information is downloaded from the system that possesses the information, and when the download is complete, the sending system cuts the connection. In the briefing call, feedback was not part of the interaction. The process of "working out something" (i.e. negotiation between the client-centric and ground reality oriented subsystems) was carried out "offline".

The cases described above illustrate "re-re-presentations" of client projects. The information that was selected was not necessarily a set of documents - a plan that was unmarked by date games and interdependent decision-making between the project coordinators and execution team 
leads - but rather a matter of conviction and negotiation. This aspect will be further developed in the following section.

\subsubsection{Negotiating client project representations}

The project coordinators must manage communication about a project with the execution team leads, who are structured orientating on the value ground reality. Being located at the main office, the project coordinators are structurally closer to the client and also in direct physical vicinity to the clientcentric client consulting teams. That this communication with the street office must be crafted differently than communication at the main office can be inferred from Preeti's reason for requesting that project coordinators be allocated a joint desk in the office:

[I]t is not good if the project coordinator in charge for the projects sits right next to the consulting person allocated to the same, which makes negotiations difficult. I can hardly say anything! Our role here is as a negotiator between the two sides. You know, before [the team was established] consulting and execution teams hated each other. So when sitting next to them [the client consultants] and talking on the phone [to the execution team leads], they suddenly intervene disturbing my conversation. For example, when I am calming a team lead that we will find a solution for the concerns on time or cost, the consultant might ask me why I am committing to anything on her behalf. But consultants don't understand that I just want to relax the situation and convince the execution team lead to carry on, to go ahead. Consultants only push - I negotiate. Because I know that the work could be done and I have to convince them [execution team leads] of that.

This is an example how the opposing value to client centricity becomes dominant in this context as a corrective value that I would also subsume under the ground reality. The client consultants operate on the basis of client centricity, which project coordinator Preeti deems incompatible with the orientation of the execution team leads' system ("push[ing]" vs. "negotiat[ing]"). This requires the project coordinators to select a different communication style with the execution team leads than that used with the client consultants. Similarly, Asif, during the escalation situation with the execution team leads, chose to continue communication outside of the official briefing call and thus outside the client consultant's awareness. The separation of the two conversation channels had to be maintained for the project to continue along its devel- 
opment process. This led to different representations of the project, which were apparently incompatible, yet relevant for the project's functioning. The different representations of the client project can hence be understood as unintentional working misunderstandings that enabled interaction across these sub-systems.

The project - as a bundle of tasks to be completed in a tight timeframe and within a limited budget - was, according to Preeti and Asif, not up for discussion in relation to feasibility; rather, it had to be believed. That these negotiations were based on a working misunderstanding of the client project (as invoked by different representations of it) becomes apparent from Rohan's position on the erroneous estimations given by "these main office people":

Rohan: When I enter into a discussion you will observe me making complaints, and main office people also making complaints. But they do not understand the ground reality. They should come here to experience this. Not only come for one visit in their AC [air conditioned] car and then leave again. Come for some weeks, come and try to do our work themselves, experience ground reality. Something like internship [here].

FM: Okay, and the other way round? Did you ever see what happens with the work your team does on it?

Rohan: No. I have never seen a presentation for client or some of analysis that happens. It will also be better for me to see what happens. Because maybe a task I think is not so important is actually very important for the client. I would be very interested on that.

Rohan uses "ground reality" here as a spatial metaphor of the hierarchical position of the street office, which occupies the lower end of the client centricity scale, as other examples have shown. He additionally indicates the street office's potential for inverting the organisation's guiding difference, as in this office, it is the ground reality that determines the possibilities, rather than client centricity. He describes the different representations of a client project, which all relate to the same need for decision-making yet are far from the same. With his request for main office colleagues to "experience the ground reality", he expresses the view that their lack of familiarity with this sub-system inhibits them from understanding its challenges for specific project situations; hence, there is an unintentional working misunderstanding. At the same time, Rohan reflects on his own lack of insight into the client consulting 
aspect of a project and, along with this, the potential for misunderstanding his contribution to it, in connection to the client.

These different representations constitute unintentional working misunderstandings due to the opacity of the different sub-systems to each other. When the client consultants refer to the "ground reality" they refer to the black box of project work tasks that are completed by the project execution teams, together with the freelancers. Representations of the clients' projects are opaque to other systems, leading to different understandings, and Rohan's own representation is also opaque. The different sub-systems must be opaque for the project to function. While client consultants, project coordinators and execution team leads process different representations of the project within their respective sub-systems, the client-centric structure of the organisation allows these representations to be related and even logically connected due to their orientation towards the client - or an idea of it. This orientation is particularly salient in representations of the "ground reality", as outlined in the following section.

\subsubsection{The boundary specialists: Organising the freelancer's work}

When I accompanied Rohan to one of his briefing meetings to the freelancers, he described to me the steps he had already undertaken for a project. As soon as he had received the official briefing call for the new project he had reviewed a list of potential candidates in his freelancer pool and contacted them via mobile phone. Within hours he had an idea of staff availability and started to plan a briefing meeting with the freelancer teams' group managers. The freelancers were, as I learned, organised into teams of four or five, each headed up by experienced freelancers who acted as Rohan's primary contacts. For this project, it took Rohan another three days to secure the availability of all of the project coordinators and prepare all of the material for the work execution process. When it was time for the briefing session, we went into the small meeting room upstairs in the street office - a five square metre windowless compartment with tin walls, equipped with approximately six chairs and a round table, on which Rohan took a seat. Five freelancers sat on the chairs, leaving one unoccupied. The rest of the room was jammed with stacks of documents and a printer. Rohan distributed a printed copy of the project briefing note, which was a cut-down version of the documentation that Rohan had received. They reviewed the document together; Rohan read each item aloud and provided a brief explanation, emphasising some of the specific requirements on 
the data to be collected, which were also described in the document. After one hour, the initial introduction was finished and Rohan suggested that the group take a short break before returning for questions. After another $45 \mathrm{~min}$ utes, the briefing ended rather abruptly. The participants would return now to their freelancers and provide them with the information about the type of data collection task to be done for this project while Rohan and I returned to the office area downstairs. Rohan's assessment of the project was that it was simple, with fairly standard requirements. He did not expect any problems because the freelancers were familiar with such work tasks and could easily accomplish them.

The project representation had been reduced to a narrow set of data collection tasks that the freelancers were expected to execute. Rohan's assessment of the project as simple and fairly standard suggests that, in his view, the different projects from various clients could be reduced to a set of standard building blocks, which would be combined anew with each project. This view is very difficult to relate back to the strategic decision of the client and again makes apparent that Rohan, as the execution team lead, orientated to the ground reality as a value. For him, a project was easy for freelancers to handle (and thus good for him) if it was simple and similar to previous projects. It is unlikely that a client consultant would have provided a similar project description (see, for example, the "most remarkable projects" described in Chapter 4, Section 4.2.1). The two values are hence incompatible. But because the client project could function as a working misunderstanding, collaboration was possible.

\subsubsection{Information selection and representation of the "ground reality"}

Representations to the environment can be observed in the practice of client visits to the execution teams. As part of the carefully managed information selection for clients about their projects with Advice Company, clients are sometimes invited to accompany one of the execution teams during small part of their workday.

This practice can be compared to a situation in Rottenburg's "parable of development aid" (2009 [2002]), in which recipients of the development aid project reports execute "on site visits" to "confront the paper-based reality with the haptic experience of the development aid projects". These visits are in Rottenburg's case, however, another set of specific and carefully managed representations of the project. Instead of a paper report, a recently painted 
maintenance shed for a recently built well is visible, but not a maintenance discipline in the project, itself. As Rottenburg asserts, this "on site visit" does not result in a confrontation with a representation of reality, but a confrontation with further representations: the wide-angled, abstract representation of the development aid project in the form of the project report is related to smaller and less abstract representations - but they remain mere representations of the reality (2009 [2002]: 93).

I accompanied a freelancer team of two ladies when they collected the data relevant for the client project. The situation I witnessed was similar to the one of the development aid project - I was presented a carefully managed representation rather than what Rohan and his freelancer teams would understand as their working reality. The individuals with whom I interacted were carefully selected senior-level college graduates and did this job already for several years, as they told me in a casual chat. The average freelancer, they claimed, would be much younger (in the early 2os) and at the early stage of his or her university education. And, no, they usually would not follow the procedures in that much detail and would not collaborate in that way. They would not work together but individually on the data collection and much faster, as much higher productivity would be required from them. Both of the ladies I accompanied were, however, "real freelancers", and this would not have been the case had I been a client visiting the street office. In such cases, as I later learned, Rohan himself would have taken over the freelancer's role. Rohan reasoned that my case was different, as usually people would "only come to check on them". But as he had heard that I was interested in their "actual work" and (from his other colleagues) that I was "okay", he felt it was neither useful nor necessary to arrange for a "normal client visit".

Hence, what I was allowed to see and experience was a specific representation of a project's "ground reality", with only partial overlaps with the perceived daily praxis of the freelancers. This representation of a project was different to the representation that clients would have seen. The representation that Rohan had selected for me was marked by mechanisms of amplification: the work procedures I observed were executed in more detail and over a significantly longer timeframe than would usually be attributed to the task. When I returned to the main office the following week I was puzzled to realise that the common representation of the client project at the "ground reality" was that of the client visit, with its numerous selection processes and amplification mechanisms: 
Even Sharan, for whose project I had accompanied the freelancers mentioned, registered surprise that I had been out and about with a freelancer team "on my own" - by which he meant without Rohan accompanying me as a representation manager throughout the day. His own experience of such scenarios was limited to client visits, during which he had experienced Rohan as a "great collaborator" who took over the freelancer's role in front of the client. In this theatre-like performance, when creating the project representation for the client, Sharan was unable to assess the processes of reduction or amplification at play.

I started to ask colleagues in the main office about their experiences of project execution at the street office; indeed, only a few had been actively involved beyond a controlled client visit. Those who had actually interacted with freelancers consisted of a handful of project coordinators who had worked as freelancers during their studies and leveraged this experience into a permanent job with the organisation, and about a dozen colleagues who had entered the organisation via a trainee programme and, through this, had spent a few weeks at the street office location. But the remainder of my interlocutors at the main office only reproduced the stories of the "ground reality" that circulated at coffee breaks, or had been, like Sharan, part of the entourage accompanying a client visit.

These examples illustrate that the ground reality opposed client centricity even with respect to representations. But they also suggest an interdependence of the two values. On the one hand, clients sometimes requested contact with the ground reality; without this insight they perceived the project as incomplete. On the other hand, this contact was manifest as a carefully orchestrated event, with Advice Company's employees - rather than "actual" freelancers - taking over the role. The client's exposure to the representation of the project at the ground reality was one that could be processed within the client's system without causing dissonance, such as by challenging the quality of work task execution or other criteria that would impede the effectiveness of the final project report for the client's decision-making. This crafted representation of a project during a client visit again suggests (as in Chapter 10, Section 10.4.2) the incompatibility of the two values and the inversion of their relevance according to the context. In these moments, the ground reality was more relevant, even for the client's system.

This section has elucidated several representations of the client project during the project execution phase within the sub-system that operates according to the ground reality value. The representations relate to each other 
and can be understood as a translation chain, in the sense of a circulating reference. Work on a client project proceeds along the project development process and unfolds the translation chain of project representations. Each subsystem attaches a different meaning to a client project and selects a different communication about it. For the client consultants, this might involve a complex array of business-strategic options; for the project execution team leads, it might involve a concrete set of data collection actions in a given timeframe. All of these selections cover different aspects of the initial reference point: the client's request for guidance around a decision. It is not despite of these different understandings that the project moves ahead, but because of the opaque quality of the non-intentional working misunderstanding, which allows for parallel encoding and therefore enables collaboration across sub-systems and locations.

In the following, I will analyse the representations of a client project at the city office and demonstrate that the working misunderstanding, with its potential for parallel encoding, serves as a key element of employees' motivation and successful project delivery.

\subsection{From data to presentations: Project view from "behind the wall"}

This section focuses on the client project's representation from the perspective of the employees at the city office. In their support roles for client consulting teams across the world, their function is to deliver outputs in the form of preformatted presentation files.

Their position in the middle of the client centricity scale corresponds with their own and others' ambiguous views on their contribution to the client project. I will illustrate how the employees in the city office reflect on their work as only marginally intellectually challenging, while positioning their function in relation to the project within the wider realms of strategic decision-making. Despite their legwork role in terms of project contribution, the employees in the city office select an understanding of their contribution that is as closely aligned with the client's decision-making need as possible. However, there is also a perception of disconnectedness and feeling of impediment, which can be summarised in a colleague's description of his position in the work process and his ability to execute as "sitting hand-cuffed behind the wall". 
Figure 24: Allocation of Section 10.5 in the project process

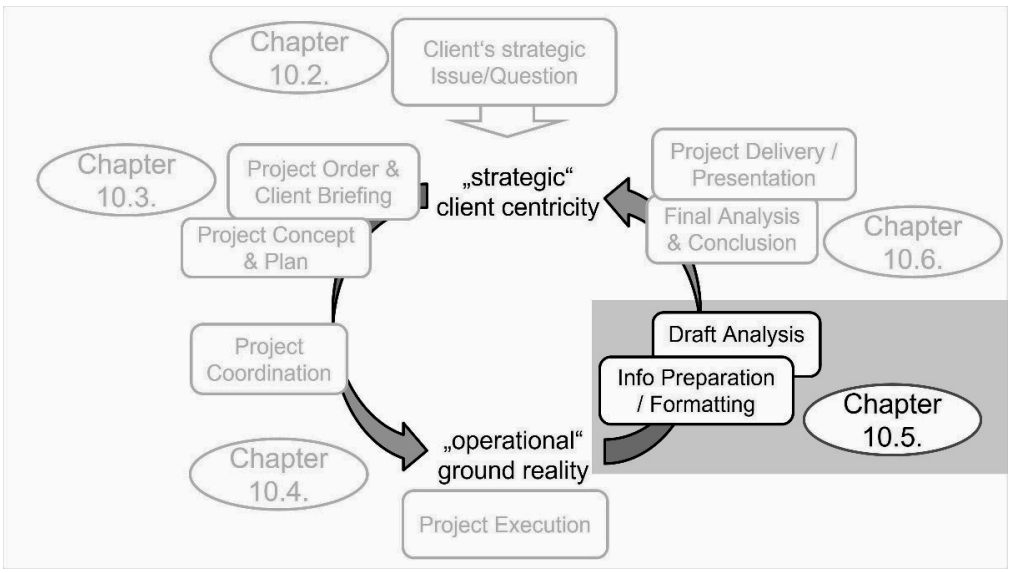

The client project as a working misunderstanding is, in this context, of central significance, as it allows employees to interpret their work as clientcentric, and this enables a more favourable assessment of their function than the work tasks would otherwise permit.

\subsection{1. $100 \%$ accurate information conversion}

When the freelancer teams complete their work tasks, the data they collected cannot be immediately processed by the client consulting sub-system. Apart from needing technical conversion into databases, which is performed by a team of experts in another city, the data must be converted into presentation slides and documents; such conversion work is performed by employees of the city office. It is of particular importance that the output exactly matches the templates and that the data is copied correctly. What comes across as simple preparation to enable client consultants to perform their strategic analysis is, in reality, an iterative series of tasks spanning several days that is executed under time and performance pressure.

For a series of three days I accompanied Amitabh, a member of the standard team, who was working on a presentation for a client consultant overseas. He had received the project briefing and started working on it only an hour before I joined him on Thursday morning. He claimed to be in a state 
of "controlled hectic", as he needed to deliver a first draft of the presentation (called a "shell") that evening. A shell is a presentation without data, analytical findings or recommendations, but with all the tables, fonts and images in their final format. This skeleton must be formatted exactly as it will appear to the client, almost like a form that will later be filled out. Once the input from the execution teams is available, the relevant content is transferred into the presentation. Amitabh was busy with this work, aligning table frames to the millimetre, placing graphics in the right corners, checking back with a colleague on the correct measures for the client organisational logo and so on. His nose seemed to almost touch the screen when he checked his work after finishing each slide. At the end of the day - at 8.30pm - he sent the presentation to the client consultant, changed his frameless, light and stylish glasses with cheap, thick and solid travel frames and equipped himself for his 90-minute commute back home.

Amitabh was in the office the next morning at 9.00am and immediately returned to his "shell" presentation, for which the client consultant had already sent him feedback. The feedback consisted of comments in little purple boxes that were embedded into the slides. The client consultant had requested that the slides be split up and grouped differently. Amitabh estimated that this work might take him the whole day. For the entire morning, he aligned images and changed the font sizes. After lunch, he continued with the presentation but became stuck in the process, as he had questions about how the slides in that part of the presentation should be set up, and in which sequence. The client consultant had described his ideas in the purple-boxed comments, but Amitabh was still unclear. Together with his colleague Nimrat he read through the comments, but they could not come to a clear solution. As the different interpretations of the instructions diverged substantially in their implications for his workload and his manager was off so could not help him with the decision, Amitabh decided to refrain from further formatting until his colleague started his workday and could advise him directly. However, as his colleague was overseas and would not begin work until 8.30pm in Amitabh's time zone, Amitabh intended to leave the office early and take the call from home. Instead, he became caught up with minor formatting tasks and the afternoon flew by. In the end, he stayed in the office on this Friday until $9.00 \mathrm{pm}$ in order to clarify what was expected from him.

When we met again on Monday morning, Amitabh told me that he had been sick with a fever and cold over the weekend and still did not feel fully recovered. "But", as he remarked, "the report has to go today. So no choice, that's 
the situation". His manager had asked the team to meet just after 11.00am to "align workloads". They discussed the deliverables of the day (i.e. who had to send what and who needed help). Amitabh announced that he could not take any additional tasks as he was still occupied with some of the formatting on his project and had not yet had the chance to work on his other tasks. Upon the astonished "Why?!?!" from his manager, Amitabh explained that the client consultant had initially sent an example presentation but now wanted the presentation to convey more details, and this involved much more work. Amitabh's manager asked him if he now knew exactly what was expected, so he could get it right when he sent the slides that evening. Amitabh confirmed that he did and returned to working on the presentation.

Up to this point, all of Amitabh's activities had been formatting and editing tasks relating to inserting empty tables with the right background colour and pasting graphics in the correct spot. At lunchtime, he finished the "shell" and was able to proceed to the next step. In this step, he copied numbers from the spreadsheet files he had been provided into the presentation file; these numbers were either absolute values or, depending on the slide context, scores that had been converted into verbal information such as "Risky", "Top", "Middle" and "Low". For the conversion ranges, Amitabh prepared a simple sheet, which he placed next to his keyboard. His eyes flew from the presentation to the spreadsheet and over to the conversion sheet, and I only heard the clicking of buttons on his keyboard when he moved back and forth on the screen, copying and pasting data in a regular sequence. His movements were fast and controlled and he worked without emotional impulse or comment.

From time to time he would pause for a moment, resting his elbows on the desk while closing his eyes and leaning over the keyboard, letting his forehead fall into his open palms. As if to justify his need for short breaks, he explained to me that this step took a lot of concentration in order to avoid typos or transposed digits. Such mistakes had to be avoided at any cost, so that they were not inadvertently reported back to the client in the official feedback. Such a scenario would have an immediate impact on Amitabh's performance rating and affect his promotion opportunities at the end of the year. This was the concrete manifestation of the department's 100\% accuracy commitment.

The copy and conversion job was completed at 5.30pm, at which point the presentation was ready to be checked by a colleague from a parallel team, to whom Amitabh sent the file. He walked over to the colleague and explained the structure of the slides and what to watch out for in the review. Amitabh's 
next and final action was "analysis" - a step that not only Amitabh, but also most of his colleagues looked forward to in the work process, and the step that was most frequently cancelled to make up time. "Analysis" involved looking at the data in the slides and summarising their conclusion in a concise caption. This was the creative part of the work - the single aspect that was not predetermined by style guidelines or data conversions. In this step, Amitabh had the opportunity to demonstrate his ability to connect the information he prepared with the client's decision-making need.

Despite his cold, Amitabh continued to work until 10.30pm on the presentation before finally sending it off to the client consultant. He could have sent the presentation sooner, without the "analysis" sentences, but this work task was of upmost importance to him, despite making up (at most) a mere $10-15 \%$ of his entire contribution to the project, in terms of time.

"Analysis" was important for most of the employees in the department, as this activity veered away from their otherwise standardised, narrow pathway of tasks. On the rare occasions that Amitabh or his teammates would get hold of the final version of the project report provided to the client, word-byword comparisons would be made to determine how many of "their analysis" sentences had been retained. This ratio was a criterion of internal status.

When I interviewed Amitabh two days later and asked him to describe his job, he did not talk about the formatting or number copying that had kept him in the office late over the past days. Instead, he substituted the pronoun "I" with "we" (referring to Advice Company) and positioned his work within the broader realms of business strategy and advice given to clients. He explained that "we" support a client's strategic decisions by providing valuable advice on business strategy. Although he did not comment or even critically assess his monotonous tasks in relation to client projects, this shift in focus during the interview illustrates that Amitabh preferred to relate the project representation more to the client than it actually was. At the same time, he emphasised his suggested insights into the projects he dealt with; these insights were most likely not explicitly his own, but inferred from the shares of information that were transmitted from the client consultant sub-system.

In this way, the client project represents a working misunderstanding between the client consultants and the city office teams. The sub-systems can collaborate because of their "parallel encoding" (Sahlins 1982) of an understanding of a client's project. The city office team rely on this mechanism, as there is only limited possibility for them to gain insight into a project beyond the information they receive via the briefing. 


\subsubsection{Amplification towards "analysis"}

Both of these points became even more poignantly apparent following Imran's emotional outburst. Imran was an embedded team member who was working on a presentation for his overseas manager. He had to align dozens of star-shaped points on a graph to indicate the development trend of a client's business. After about an hour, he sighed and his head sank down, stopping an inch before hitting the keyboard. Two seconds later he turned his head to me while remaining in this position, looked up and said: "This is just the most depressing work!" Although his theatrical outburst was so amusing that I couldn't help but answer it with a smile, his voice and expression were serious when he straightened up again:

„You know, it is the most stupid task to do, putting squares on squares for hours. And when you do that all these depressing thoughts come to your mind, because you don't have much to do, such as "Why am I doing this job"... Look!". He kept moving stars around the presentation slide. "And they do not even want to stay that way!!" He bristled in anger when the stars did not align the way he wanted them to, and shook his head: „No, it really is the most unproductive work - my head is almost paining because of the stars. And I am not even sure if this really is what she [the overseas manager] wants in the end. At first she indicated I should not pay attention to formatting, so I sent her my analysis of the material and now it is only about formatting."

Imran remained busy formatting the presentation slides for most of the afternoon and the following morning. I realised that I, too, was equally bored and impatient just by sitting next to him. But suddenly, with an accentuated gesture, he reached for the computer mouse and my attention turned back to him. With ostentation he saved his work, energetically rose from his chair and indicated with a move of his head that I should follow him to the canteen. There, he told me over a chai that he was finally done with the "stars job".

When we returned to his desk, he sat down and stretched his upper body and arms as if preparing for a race. Then he leaned forward, rubbed his hands and wiggled his fingers in the air like a piano player. With a broad grin he looked over to me while announcing with raised eyebrows: "And now - analysis!!" Then he explained what he thought the interpretation and argumentation of the client report should be, as well as the recommendation to the client. He dove into the slides for a good hour, formulating table headings and developing alternative colour coding for the graphs. 
When he completed his analysis and sent the presentation off to his manager, he told me about a previous project he had worked on that had been "much more creative", and he showed me the appreciation he had received for it. The client consultant had written in the feedback email that he "helped shaping the final project report to win the clients satisfaction". That email was positively commented on and acknowledged by both his local team lead and the division head. When I did not seem to display his expected reaction to the division head's email he pointed out to me who had sent the email, in case I did not know: "You know who that guy is? He is veeery senior. [He lowers his voice to a whisper] It's from that guy in the office in front, the Vice President."

The next day Imran showed me the email response from his manager about his work on the presentation. Although she was still demanding a number of changes that would keep him busy with another round of formatting for at least the rest of the day, he was content with her email, as it showed that she acknowledged the effort he had put into the presentation over the past few days. I asked how he had come to that conclusion, as I did not see any words that explicitly mentioned this in her email. He said that her tone was a bit softer, as it was normally very strict and commanding. He pointed to places in which - in comparison to her other, more neutrally phrased, emails - she had used words that mimicked spoken conversation - "Aaaaah, now I see" and "Hmmmm, not so sure about that" - as direct responses to his text. To Imran, this indicated that she was aware of his efforts and the issues he had encountered, as she repeated the explanations he had given in the email that accompanied the draft he had sent her the previous day. But an even clearer indicator was for Imran the fact that she had used the smiley emoticon - :) twice. All of these indicators, in conjunction with her suggestion at the bottom of the email that they could "have a phone conversation at some point" about the project, indicated to Imran that he had made a breakthrough. Imran proclaimed with a smile: "Now she is at the point where she is ready to talk with me."

While slightly more explicit than the first, this second case illustrates a similar disparity between the work that was predominantly associated with the project contribution and the imagined focus of this work. Imran clearly expressed his frustration with the formatting tasks that were expected of him and contrasted these to the more highly valued "analysis", both implicitly through his energetic attitude as soon as he could proceed to "analysis" - and explicitly - through his reference to the appreciation email. What is noteworthy is that the appreciation email did not distinctly compliment his analysis, 
but more broadly supported him in "shaping the report to the client's satisfaction". Following the overall client-centric orientation of the organisation, Imran attributed the appreciation to his analysis work - a task that was more closely associated with the client's decision-making need than was presentation formatting.

The role played by the "analysis", in contrast to the editing tasks, at the city office is even more accentuated when one considers the office's position in the middle of the client centricity scale. While the sub-system Imran and his colleagues at the city office belong to is apparently structured on the basis of client centricity as the dominant value, their function is rather to relay between ground reality and client centricity. Formatting presentation slides and copying data from databases and spreadsheets makes the information provided by freelancers - the ground reality - usable within Advice Company. In the most literal sense, the information is internally reproduced through these presentations, and the target is to achieve $100 \%$ accuracy in this reproduction. For the editing and copying tasks, the ground reality is therefore the prevailing value - at least according to the department manager's view of the city office. The "analysis" task, in contrast, is orientated towards client centricity. Without "analysis", the presentation is merely reproduced information from the ground reality that cannot be processed by the client's system and therefore requires further transformation. "Analysis" is accordingly a client-centric task that corresponds with the city office employees' self-description.

Figure 25: Balancing tasks at the city office

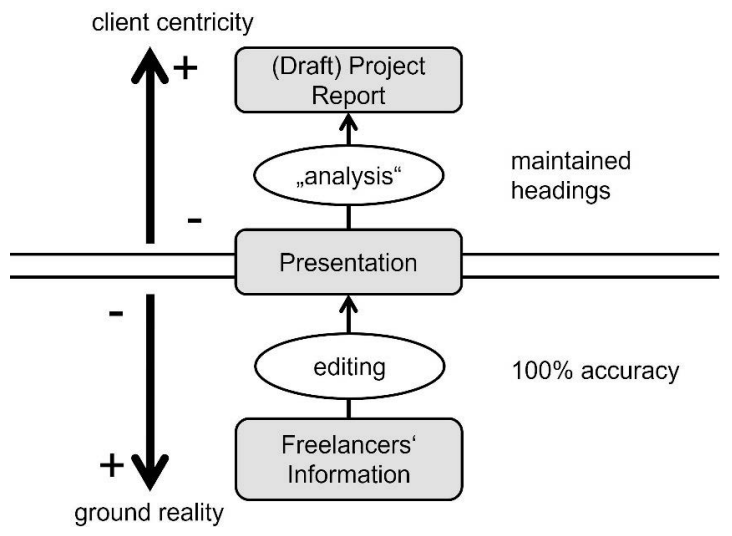


Similar to the project coordinators, the city office employees must manage two incompatible values, as the two cases have illustrated (Figure 25). But the employees have developed strategies to keep the two values separate, by communicating the difference between tasks. Imran explicitly marked the end of the editing tasks and even changed his location through a break to highlight the beginning of the "analysis" phase. The number of "analysis" headings that prevail in the final client report is communicated as an indicator of status amongst the employees (while an error-free presentation is not), and Amitabh did not even mention his editing tasks when he was interviewed about his job. Thus, the activities relating to the different values are maintained as separate and the system remains in function.

\subsection{3. "Handcuffed behind the wall"}

The reading of a client project as a client-centric activity centred on analysis was prevalent amongst the city office employees, and this related to a notion of their position as peripheral. The case study of Imran shows that he worked with the project representation he was provided with, and he had to cope with his distance to the actual client consulting team. With his interpretation of his manager's email, he attempted to establish an idea of closer interaction - a closer position (of himself) to the client consulting function, invoked by means of communication. It was his hope to overcome the key issue that a client consultant might not brief him on all of the important aspects of a project and what the client really wants, "because she might forget some aspects in the briefing, but if I sit next to her she might remember and say something later".

This inclination towards the client, together with the willingness to make a successful contribution to the client, was also voiced by Ananya. She told me about a situation that had occurred a few days earlier when a client had raised a question about a report that had been resolved by the client consultant she supported in a major Asian capital city. She had only heard about the client's question today, several days later, and only by chance in the team call. She was convinced that she would have been able to resolve the issue and to answer any tricky questions the client might have. But, as she reasoned: "I am too far away, I can't action and when I hear about it, it's done already. So I can never really prove myself and my real abilities."

Her colleague Anas told his teammate about a project that had received a major review request from the client and resulted in tremendous cost for Ad- 
vice Company because a portion of the project work had to be redone. According to Anas, this had occurred because the client consultant had been unable to clarify the questions that had risen from the report. Anas remarked:

I so much wish I had been there and heard about the questions from that guy. I am sure that I might have been able to tell the client a solution to his question and with this have saved the company a lot of bucks! [He emphasises the second sentence with a punch of his right fist into the open palm of his left hand.]

Niharika explained this notion of disconnect through the example of a project for which she did not receive positive feedback, because she had not been told about a shift in the client's focus. Thus, she had not been able to incorporate the new focus in the presentation. She complained about the unjust evaluation but actually wanted to make a different point when telling the story:

My general point is that the analysts in the US are in constant touch with their top-level managers who know what the client wants and how the report should be done. So the client consultant gets that learning from the manager through experiencing the update process of the report and their conversations and so on. But this information never gets back to me, I never get to hear about it. And with the next project I am supporting I have the feeling all [colleagues] over there have made a shift closer towards what the client is expecting there in the US but I haven't.

Sameer, who primarily supported colleagues in the main office, summarised his view of disconnectedness in this way:

We are all here working for a remote team and it is them [the client consultants in other offices] who actually have the projects and we are all somehow behind the wall. I feel a bit like handcuffed; I cannot give my best, as I would if I was there and on the project right from the beginning. But I don't know the background of the project and so on.

These accounts illustrate the notion of the city office's peripheral position, away from the client-centric functions. The office's (functional) distance to the client-centric consultant teams make it depend on the information that is selected by the client consultant managers. The employees' interpretations of their work are marked by a notion of disconnectedness and an inability to work as closely to the client as they feel they are capable of. As I showed in the previous section, their interpretation of their position is more aligned 
with the organisation's dominant value client centricity than their function. The overseas managers' view of this situation will be shown in the following section.

\subsubsection{A perspective from in front of the wall}

During my research stint in the city office it was Cory from one of the overseas offices - who had combined a holiday with a few days visiting colleagues in the city office - who enabled me to view the project work of the city office teams from the other side of the wall, from a Western, client consulting perspective:

FM: How is your interaction with the team from abroad?

Cory: In the first six months communications were primarily via email and with filling out that spreadsheet with the required work and the needed info on the project. You could almost compare this with an order form: you enter what you need, send it across and the work gets done. If something is completely unclear they [the city office employees] might ask, otherwise they just do their tasks on the report until they send me the first draft. And to this I also send the feedback via email and comments in the slide deck. Only recently I have started to chat more [via the Advice Company internal instant messaging program] and sometimes even make an IM call [instant messenger voice call] at the end of my day to say what I have done today and what I expect them to do during their day, so that it is done when I come back in tomorrow.

FM: Where does your "real life" experience of being here deviate from your expectations?

Cory: Well, I did not really have an image or an idea of individuals here. The interaction for me in the past was very transactional: you fill out the form and you get the work done. It is difficult to understand that there is someone, a real person actually doing that work. And actually, the workload is mostly very high on my end, so I just don't have the time to think about these things. The work gets done, it saves you a few hours' time, but basically you're consequently asked to take up more projects. At my office there is the direct pressure from clients, more work pressure I think. And compared to here it is all much more, like "heads down and working", you know. 
FM: Where would you say are the main areas of collaboration or "working together"?

Cory: $\mathrm{Hm}$, it is in my opinion not really "working together" since they would hardly speak up and always try to fulfil my idea rather than their own. But being here, I am actually surprised how, you know like... similar people are here to myself.

Cory's perspective allows for a number of inferences to be drawn. First, it is clear that the formatting and data transformation delivered by the city office teams are a black box for him. He does not initially reflect on the fact that an individual colleague completes the tasks he orders via a form. The lived working praxis of Amitabh, Imran and Niharika, with their struggles to give meaning to the endless hours of dreary formatting tasks and their notions of disconnectedness from the client-centric system they see themselves as working for, remain invisible to him. Cory - and most likely the majority of his colleagues - is equally unaware of the city office teams' internal value system of work (distinguishing "analysis" from editing) and their desire to establish a new layer of relationship beyond that which is structured by the presentation delivery. By comparing the project briefing to an order form and referencing the client pressure he is exposed to, Cory confirms the organisational structure along the client centricity scale. His position is close to the client and the order form procedure is justified by his high workload. In his view, his city office colleagues are indeed "sitting behind the wall" and their work on projects is only necessary for completing draft presentations.

\subsubsection{Working misunderstanding for motivation}

I have illustrated in this section how the work on client projects in the city office is marked by the challenge of managing the two opposing values ground reality and client centricity. The expressed feelings of insufficiency relate to the fact that only the "analysis" work on a client project at the city office is attributable to the value client centricity. However, the majority of the work in this office is spent reproducing the information that is provided by the freelancers; that is, reproducing the ground reality by formatting and transferring data according to a $100 \%$ accuracy commitment. Hence, the organisational performance measure orientates the function to the ground reality 
value. But in Advice Company, informal prestige and motivation is gained from tasks associated with client centricity, such as "analysis" work. The employees' strategy of keeping the incompatible systems separate creates a "rere-representation" of the client project along a mechanism of amplification: despite devoting only $10-15 \%$ of their work effort to "analysis", the employees view this single aspect of their work as their primary source of motivation. Amitabh did not even mention the other $85 \%$ of his work effort when I asked him to describe his job, and Imran attributed the appreciative email of his manager to his analytical skill. This ambiguity in the project representation - allowing room for the employees to ascribe their work contribution to a client-centric category - is the working misunderstanding that maintains the structural set-up. While Cory views the same tasks differently, the parallel encoding of client projects enables the collaboration between the city office employees and the client consultants across the world.

\subsection{From presentation files to strategy}

In this final section I will focus on the last phase of the client project process, when the project report is finalised for delivery to the client. This step is performed by the client consultants at the main office and delineates the moment at which a saleable commodity is created (Figure 26). This perspective completes the insights into the black box of the "client project".

At the end of this final step of the project process stands the delivery of the project report. This is the moment when the client's need for decision-making is confronted with its representation at the end of a long translation chain. The project report is finalised by the client consultants only after the various representations of the client project have served as the basis for collaboration across the organisation's sub-systems and undergone mechanisms of amplification and reduction. I will show in this section how the last phase of the client project is marked by another set of representations that are dependent on a non-intentional working misunderstanding. To illustrate the contextual meaning of time pressure, the analysis commences with a change of perspective on the client project from a function-centred to a project-centred view. I will continue to examine the processes at play within the client consulting teams around the project analysis. In a concluding section, I will discuss the client consultants' strategy for maintaining the black box of the project for the client, drawing on opacity as an organisational strategy for self-sustainment. 
Figure 26: Allocation of Section 10.6 in the project process

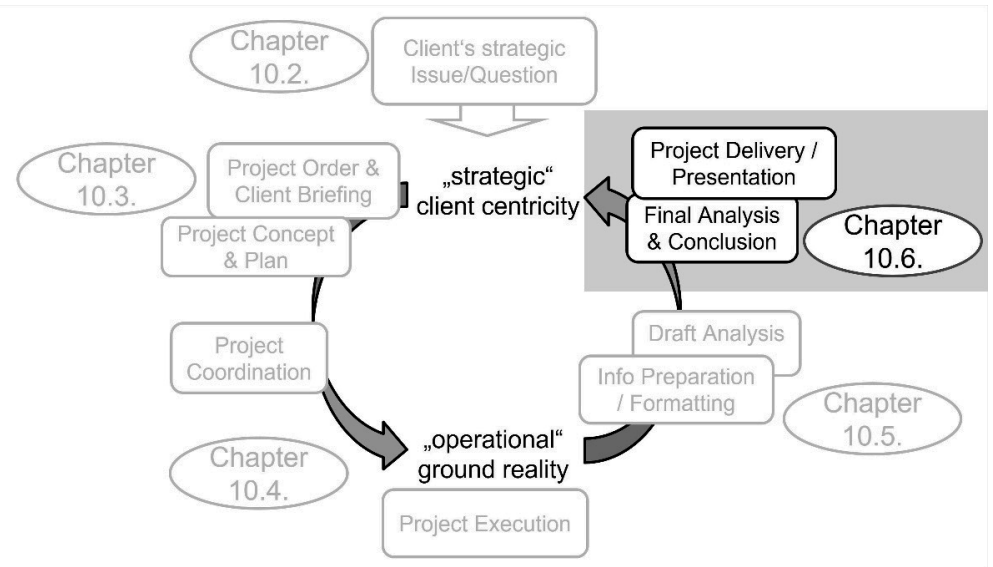

\subsubsection{Deadlines from a project perspective}

In Chapter 5 (Section 5.4.1) I described the dense sequence of communications and the different topics the client consultant Ruchika had to address on a "busy day". Cory, the overseas client consultant, was shown to mirror this praxis in his description of the city office employees as "heads down and working" (see Section 10.5.4). This perception corresponds with the buffering strategies of delivery date games played by the client consultants with the clients and project coordinators to ensure the timely delivery of project reports (see Chapter 9).

Hence, I was prepared for a similarly dense and high-action experience when embarking on the quest to follow three client projects through the project development process in the final phase of my fieldwork. The change of focus proved useful for generating a wide array of insights into the emic perspective of the working praxis at Advice Company, which I have already discussed with respect to a working misunderstanding around notions of collaboration between myself and my interlocutors (see Chapter 8). But the most relevant aspect for this section was the insight that the projects I had accompanied were far from resembling the steady and intensive demand for task completion that characterised the client consultants' daily work praxis. Two of the three projects I accompanied reached the final report phase during 
the two-month timeframe that was allocated to this last fieldwork period. I had permission to track a third project, but this project was postponed indefinitely due to severe issues encountered in the middle of the execution phase relating to an ongoing project with the client. The following vignette of the final phase of project GREEN illustrates the project as understood by the client consultants.

Kashish was the client consultant for project GREEN. The project was in its final stage and the deadline for delivery to the client was approaching. On the 2nd of May, Kashish told me that he had received the first draft of the report and would now have to prepare a cut-down preview version to send to the client in a week's time, on the 9th of May, and would need to deliver the full presentation on the 15 th. As he told me, he still had plenty of time on that project and many other things to do and deadlines to meet. Hence, he would not work on the project immediately, but might spend a bit of time on it sometime in the next week. I approached Kashish again two days later, on the 5th of May, and he told me that he might work on the project that afternoon. We agreed to meet up for a coffee and a chat after lunch and that I would accompany him for the afternoon. But when I returned to his desk a few hours later, a more urgent task was on top of his list and he did not look at the project GREEN report at all. The situation remained the same the next day (6th May). When I came to Kashish's desk the next morning, on the 7th, he told me that the project would be handed over to Neelam that afternoon, as he had too many other things on his plate to take care of. He would send Neelam an email with details of what had been done thus far and Neelam would take care of the project. While a lot was going on from Kashish's perspective, the progress on project GREEN had been slow - if indeed there had been any during the previous days.

On the following morning, 8th May, I therefore joined Neelam, who sat a few desks away from Kashish. Neelam told me that he did not have the slightest clue about the project and that he needed to see how he could get it done. Using chat, he asked his manager Aishwarya for a clarification call at 2.00pm. I asked why the project had been transferred to him. He answered that he might have "some free time and Kashish is blocked". "See, my work plan for the next 12 days, it is okay." He showed me an email containing a list of 12 deliverables, before taking me through his tasks for the next days:

Now at noon, I have to send this presentation for a different project, but see, it is almost done, I just have to add a few comments. Then at 2.00pm I can 
connect on that CREEN project with my manager, at 2.30 I have to leave for a client meeting. Then I will be back here around 5.30 and work on the other presentation which will have to go tonight. Will be a long evening... And then, on Friday noon the preview report for GREEN will have to go and then I have to finalise another presentation which I have to present at the client's office on Monday. This I will send on Friday EOD [end of day] to my manager and the team lead for feedback and incorporate this hopefully on Saturday so that I can relax on Sunday.

After almost a year's fieldwork at Advice Company, I was still astonished to hear Neelam's definition of "some free time", though I tried hard not to show it. Shortly thereafter, as Neelam concentrated fully on finalising the presentation (which was due in a few hours), his teammate Swati walked past on the way to her desk. She looked at me and smiled: "Ah, so you're with Neelam now." I explained to her that I came with the project, which had been handed over from Kashish the previous night. Her smile froze and she leaned towards Neelam, asking: „Which project?“ „This GREEN project.“, he replied. Swati's eyes widened. Then she straightened up, raised her eyebrows and twisted the palm of her right hand up in a typical move indicating a non-verbal "What?" or "Why?". Neelam answered her unarticulated question.

Neelam: Nobody told me about it, just Kashish told me last evening.

Swati: With whom did he talk about that?

Neelam: With Aishwarya.

Swati: And she has spoken with...?

Neelam: Ashish [the head of the team]. [He continues in a lower voice] The inputs from the other teams are said to be okay, but I asked Kashish - not much happened so far.

Swati: So you got the project yesterday. When is delivery date?

Neelam: On the 15th... but preview has to go tomorrow.

Swati: Tomorrow? [Her eyebrows rise up again and she frowns.] 
Neelam: Yeah, well, first draft is there, I will connect with Aishwarya post lunch and will get one of the interns to help me on it.

Indicating resignation, Swati raised her gaze to the ceiling and remained in that position for a few seconds. Then she gave me two light pats on the shoulder while she turned towards her desk and walked off without another word.

During lunch at the canteen with the entire team, Neelam rose from the table shortly after $2.00 \mathrm{pm}$, announcing with a frozen grin towards Kashish: "Apologies to all, but I got to get up. I have an important call to make." Kashish smiled broadly and said: "No idea what you're talking about..." But everyone at the table knew that Neelam had to leave to call his manager to gain a better understanding of project GREEN, which had been handed to him from Kashish.

After a short phone conversation with his manager about the project, Neelam left for a client meeting and returned around 7.00pm. He immediately started to work on the preview report on GREEN for the next day's delivery and detected an issue with the argumentation in the recommendation. He called Kashish over and both discussed how the issue could be mitigated. At $9.00 \mathrm{pm}$ they reached a solution and Neelam decided to go home to work on another presentation that was due that evening. He would finish the GREEN presentation later. When he came to office the next morning he told me he had worked on the first presentation until $12.30 \mathrm{am}$ and got up again at $4.00 \mathrm{am}$ to bring the GREEN preview report to a status he deemed sufficient to send to his manager for review. Then he went back to sleep for another three hours before arriving back at the office. This was his routine when he had work to do, he explained to me. That day, he would finalise the preview report on GREEN with his manager's comments and send it off before attending to the next project deadline of that afternoon.

While Neelam booted his laptop, his manager Aishwarya approached from the other end of the office. She announced that the preview report on GREEN could not be shared with the client that day because the client had not yet paid the minimum deposit of $50 \%$ of the project cost. The finance controller had just given her the latest payment status for the team and the payment had not arrived as of that morning. She would raise this with the team lead later, but for today, the report definitely would not go out. This was discussed for a few more minutes, before Neelam re-arranged his time plan. He agreed to meet up with Swati in 15 minutes so they could work on the presentation due that afternoon. The project GREEN deadline had disappeared, and it had 
immediately slipped off Neelam's focus. It did not come up again until the middle of the following week, when the payment situation was resolved.

Besides providing an impression of the working praxis of one of the client consulting teams, this case illustrates that a project viewed as a single entity entails different dynamics than the client consultant's praxis in the project context. The client consultant's task list is determined by immediate project deadlines: the more presentation deadlines, the higher the workload, and workload seems to fluctuate at a steady continuum between high and very high (as perceived by the employees). Consequently, I expected the work associated with a client project to be densely packed. But instead, I observed in this last phase of the project a fascinating contrast: as soon as all previous tasks had been completed and finalisation of the report was the only action pending, the work associated with a project would converge towards zero for days or even weeks. This was shown in the example of project GREEN, when deadlines for other projects overlaid Kashish's to do list until project GREEN's approaching delivery date augmented its position on the priority list.

In light of the upcoming delivery date, work activities on the project surged from zero one day to Neelam's nightshift the following day. While the project presentation remained literally untouched for days, it was progressed within 24 hours to a level at which it was almost ready to send to the client. An ironic twist occurred when the deadline evaporated due to the client's outstanding payment: at this point, Neelam's attention instantly redirected towards the next deadline and progress on project GREEN fell to zero again.

This effect became salient only from the viewpoint of a single project. Earlier in the fieldwork process, when I accompanied client consulting colleagues throughout their workdays, only their struggle to meet the sequence of deadlines for different projects marked the character of this final project phase. Now my observations suggested that the overall duration of this final project phase was predominantly determined by delivery dates and not the actual work required to make final recommendations. These mechanisms of reduction occurred at the last node in the project translation chain. Paradoxically, this meant that the most relevant activity for transforming client projects into saleable commodities was frequently performed under tremendous time pressure by the client consultants, even though a sufficient margin of time was built into each project. Furthermore, project analysis and recommendations, which comprised the most prestigious tasks in the organisation, took only a marginal amount of time, both in relation to the entire work involved in a client project across the organisation and in relation to the total 
activities performed by a client consultant. Another noteworthy aspect of the example of project GREEN is that Kashish passed the work on to Neelam, who was not thrilled to receive it. While Neelam did not admit this directly, it became apparent in his short conversation with Swati and his brief verbal teasing of Kashish at lunch. The finalisation of the project report was, in this case, yet another unpopular task on the list that the team manager had distributed to another team member.

Giving advice to clients - explaining an approach to a successful strategy - is the image of the client consultant job at Advice Company and the work that other employees mention as their career target. But this work appears in everyday praxis as a well nurtured working misunderstanding and a well-sealed black box for clients. The fact that the actual time spent on clients' advice is minor in comparison to the overall duration of a client project allows for an interesting parallel to be drawn with the working practice of the city office employees. As I showed in Section 10.5, the city office employees use the mechanisms of amplification to frame an understanding of their job according to the client-centric task of "analysis" work, in contrast to the editing tasks that actually comprise the majority of their work. The next section will show how the client consulting teams also use processes of amplification both in their finalisation of project reports and their perceptions of their work tasks - similar to the city office employees.

\subsubsection{Analysis as a process}

Despite the time pressure under which project presentations are often finalised, the process is marked by a rigid sequence of review steps along the hierarchical structure of the client consulting teams. Before a project report is actually provided to a client, it must follow a sequence of review and input. Client consultant Raveena explained to me that she usually provided a first draft to her manager, who would give her feedback that she would incorporate before sending the presentation to the next level - the team manager Ashish. Ashish's feedback would also be incorporated into the final version before it was shared with the client. For big (i.e. high budget) projects or important clients, sometimes even the division head would be consulted for feedback, and this entailed a different level of complexity. Together with the deadline effect described above, last-minute nightshifts seemed inevitable.

The function of this process can be seen as another mechanism of amplification at this last node in the translation chain. The involvement of senior- 
level employees in the final information selection for the client communication underlines the importance of this activity and affirms the organisation's structure along the client centricity scale. The presentation review chain reveals the nature of the client project as a non-intentional working misunderstanding, as illustrated in Raveena's assertion that thought processes - and especially recommendations - differ from person to person. For this reason, presentations undergo multiple reviews and iterations until the moment they are delivered to the client, sometimes even in the car on the way to a presentation at a client's office.

Sunidhi, a junior client consultant, described the most difficult situation for her as when the division head, who was four hierarchy levels above her, spontaneously gave her feedback on a report she was working on. I had observed this situation multiple times: the division head had the habit of appearing at a desk and asking what a colleague was working on. Then he would look at the client question or project briefing for a few seconds and explain in three to five minutes (often also drawing on a note pad or piece of paper) how the argumentation should run. He would refer in these moments back to successful project presentations with similar cases. Then he would continue to the next person or his mobile would ring, and the interaction would be concluded. Sunidhi admitted hesitantly in our conversation that she never knew what to do with this feedback. She felt too intimidated to reach out to him ${ }^{5}$ to ask him to clarify, so instead she tried her best to guess his meaning.

\subsubsection{Playing on the black box}

All of these procedures and practices remained invisible to the client when he or she finally received the recommendation for the pending decision in the client's organisation. The need to keep the project a black box with only the recommendation output revealed is relevant insofar as Advice Company's recommendation serves to support clients' decision-making processes. For this purpose, ambiguities around different viewpoints on a project and the various mechanisms at play in the translation chain must remain invisible. At the same time, the consulting teams can utilise this situation, as the following example illustrates: 
Over lunch Raveena recalled an incident that had occurred two months prior, when a client had asked for clarification on a project they had just delivered. Although Raveena and Bright discussed the problem for hours, as of 10.30pm they had not reached a satisfying answer to give to the client. In the early morning, they continued to discuss the problem and finally involved the division head. As they had to provide an answer at noon, all three devised an email with a number of points addressing the question and an answer they deemed would be well received. The final claim in the email was that "the data and information available supports that", and the email was sent. The group waited in tension for the rest of the day to receive the client's reaction. It never came. Apparently, the client was content with the answer and did not wish to dive for deeper insight into the black box.

As Raveena was retelling the story, I could infer that it had been a remarkable incident for her and that the integrity of the black box was important, both for her and for her two colleagues. Second, I concluded that mere reference to the black box of analysis by the client consulting team was sufficient for the client to accept the explanation.

\subsection{Concluding remarks on working misunderstandings}

Part II has focused on working misunderstandings and their productive quality for facilitating social interaction. Based on a literature review, I have shown that the concept of working misunderstandings has been applied across various disciplines over the last five decades and that the diverging applications of this concept in ethnographic research can be positioned on a quadrant model consisting of two analytical dimensions: differentiation of the involved parties (locus) and level of intentionality (modus). Relating to the L/M quadrant typology, the chapters of Part II have analysed working misunderstandings in the context of client projects at Advice Company according to each of the four categories in the model (Figure 27).

The working misunderstanding around notions of "collaboration" between myself and my interlocutors uncovered differing ideas of collaboration practices and approaches to information selection (see Chapter 8), illustrating that working misunderstandings in that locus category bear opportunities, rather than threats, for ethnographic insight. These notions of collaboration formed the background for Chapter 9, which sought to understand the complexities behind "date games" as intentional working misunderstandings beyond 
Figure 27: Part II categories of working misunderstandings

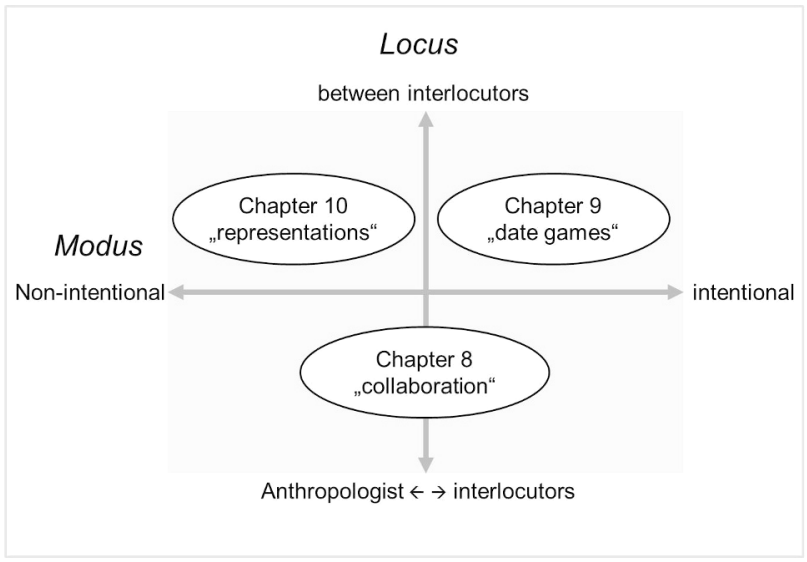

seemingly rational interdependent decision-making. While the date games are structured on the basis of the organisation's prevailing value client centricity, the intentional working misunderstandings during the execution phase (relating to "status updates") suggest an inversion of the values' relevance in this context towards ground reality - at least until client-centric escalation strategies sever communication. This intentional modus of working misunderstandings provides a different and counterintuitive perspective on the relevance of the value ground reality for organisational functioning. The limits of intentionality within this category of working misunderstandings are, however, not sharply defined. The question of how we can draw a line with respect to practices of deception or "misrepresentation" (Strauss 1993: 182) is one that invites a wider focus on working misunderstandings as a research field. However, the systematic classification of working misunderstandings along the two dimensions of the typology quadrant allows us to solidify such questions.

The non-intentional modus of working misunderstandings was the focus of Chapter 10, in which I traced the different representations of a client project. Resonating with Latour's concept of the circulating reference (Latour 2000), these representations were shown to be shaped by amplification and reduction at each node in the translation chain along the project development process. A client project comprises a working misunderstanding, as 
it allows for interacting sub-systems to "attach" their representations of the project without impeding the communication required for organisational functioning. The analysis of a client project as a non-intentional working misunderstanding enabled further insight into the black box of organisational functioning. I illustrated how the opposing values client centricity and ground reality are incompatible and that working misunderstandings keep them operationally closed whilst enabling interactional openness. The cases also showed that the relevance of the two values is reversed at several points in the project process. This assertion supports earlier inferences suggesting that both values are required for a successful client project and the existence of Advice Company as an organisation.

I have shown in this Part II of this book how the L/M quadrant structure can be applied as an analytical tool for ethnographic insight on the complex topic of working misunderstandings. Hence, I propose this model as a starting point to foster a streamlined discussion leading to coherent new terminology and/or a new analytical direction for working misunderstandings. 


\section{Conclusion}

This ethnography has centred on the two research questions of how misunderstandings shape an organisational system and why they can be understood as a productive element of organisational functioning. Based on Luhmann's Systems Theory I identified communication as the constitutive operation for social systems and presented working misunderstandings as a crucial phenomenon for maintaining the flow of communication and the function of the organisational system.

My conclusions have built on ethnographic cases of the interactions, conversations and events I observed (and sometimes also participated in) between February 2013 and June 2014 in and around the offices of Advice Company. Some of the situations described, concerning the organisational structure, the hierarchy and status of job types and the collaboration and interaction processes, are probably very familiar to the readers. As most of us are members of a complex organisation, readers may find that the practices described here resemble their personal experiences - even if the analytical perspectives might suggest new and sometimes counterintuitive points of view. Other case studies might seem unusual or different, though these again may describe situations that are similar to those encountered by readers who have ever started a job in a new company or university. Those who can recall such a moment most likely vividly remember the challenging first weeks of adjusting to a different organisational framework and discovering its tacit ways of working without falling into its many pitfalls. Consequently, some of the practices and structures I have analysed might be similar to those that recur across many MNCs in the professional services sector. Others might be unique to Advice Company as an organisation. It is difficult to draw this line. However, an analytical focus I deliberately avoided was the role played by the Indian location of the fieldwork. 


\subsection{How "Indian" is Advice Company?}

Advice Company is an MNC of Western origin that launched its Indian operations during the country's economic liberalisation in the late 1980 s and early 1990s (Nayak 2011: 38). Following economic reforms that derestricted governmental regulations, both Indian organisations and foreign MNCS sought to benefit from the emerging markets and opportunities. Although several foreign MNCs (such as many in the steel sector) were already operating plants in India at that time, the early 1990 s led to a significant increase in global players within the consumer goods and services sectors in the Indian market (Mazumdar 2012: 28-31). The offices in which I conducted my fieldwork are located in an Indian megacity that was referred to by my interlocutors as "the country's cultural melting pot". On the basis of my own experiences working at regional head offices of MNCs in capital cities around the world, I had expected to find several nationalities amongst my interlocutors, at least at Advice Company's main office. Instead, apart from two European employees who had migrated to India several years back and a handful of colleagues from India's direct neighbouring countries, all of the employees, contractors and freelancers I interacted with were Indian citizens. They came from all over India and represented groups that could be connected to the Indian middle classes, with their "knowledge based occupation, a career-oriented work-culture and valorization of education" (Panini 2015: 19). In particular, employees who were younger than 35 were also international travellers; some of them had lived, studied or worked abroad for extended periods of time, much like myself. In light of this organisational framework and my interlocutors' presuppositions, the question arises: What role did India and a group of interlocutors who were almost exclusively Indian play in this research?

Studies of the IT services and development industry have emphasised differences in the organisational practices of Indian IT companies, relative to their European counterparts (Upadhya 2016); the different use of workforces between German and Indian MNCs (Mayer-Ahuja 2011a); and the differences in workplaces between Indian and German companies (Gupte and MüllerGupte 2010). Drawing on dimension-based models of organisational culture, other works have considered MNCs "not only a manifestation of globalisation", but also "an embodiment of the fundamental values of capitalism" (Shah 2015: 37), and voiced the hypothesis that employees of MNCs in India show lower levels of social/family collectivism than their colleagues in Indian organisations (ibid.: 44). Similarly, one study sought to trace which "Indian societal 
values influence the organisational culture in three MNCs operating in India" (Khandelwal 2009: 125) through an application of Hofstede's dimensions of individualism/collectivism and high/low power distance. As I was unable to gain insight into Advice Company's work practices at their overseas offices (apart from the limited insight provided by Cory's perspective on the city office, as discussed in Chapter 10, Section 10.5.4) and brought forward my concerns to employ dimension-based concepts relating to the metaphor of "organisational culture" (Section 2.2), it would be presumptuous of me to even attempt to answer the question of how "Indian" Advice Company is. Instead, I asked the experts - my interlocutors - during interviews and informal conversations about the extent to which Advice Company could be considered, in their opinion, an "Indian" company. It might not be surprising that their answers turned out to be as differentiated as the complex organisation, itself. The following selection of quotes seeks to convey an impression of the employees' answers across the different offices, job types and work experiences:

Advice Company is very different to an Indian public sector bank. There you can find the typically Indian office. People leave at $4.30 \mathrm{pm}$ if things are done or not. And there are rigid hierarchies. Here is more a modern environment with different [longer] work times and more open attitude.

If you want to see Indian work culture, then you have to go to government offices. Women wear sarees and the senior-most person never works - they get the work done from juniors. Here at Advice Company it's not that way: everybody works. And here's no attitude, you talk to your manager and even the director.

Before I joined I expected a cold and distant climate at an MNC, but I found friends and a good work environment.

You can see it's an MNC because there is no "Sir" or "Maam" culture. But we kind of follow hierarchy here that would be more Indian. I worked with a different MNC before and we did not have this there.

Any office in India would just be like that: the boss plays a key role, we have a high dependency and orientation on the manager. 
It's a typically Indian work culture: there is no law. You can make the person work for more than the required time the person is paid for - and it's still okay.

People here rely less on processes and more on people. Every client has a one favourite employee they can rely on. That's typically Indian.

In spite of this diverse set of statements, however, an implicit idea of "Indianness" in the context of business organisations is conveyed. This notion of "Indianness" manifests itself in the image of public sector and government offices, which is contrasted with a fictitious process-orientated Western organisation with fewer traits of the "Indian office". Only one of my interlocutors, who had briefly lived in Tokyo, mentioned other organisational contexts. This interlocutor reflected on Japanese firms' lifetime employee perspective, which stands in contrast to the higher rate of job changes in India.

The quotes from other interlocutors centre on shared ideas of "typical Indian offices" relating to hierarchical power relationships in various manifestations and levels of sociality. Rigid hierarchies with impermeable communication chains were perceived as opposed to the modern "open attitude" and approachability of managers, as represented by the use of first names in MNCs. The employees thus considered "Indianness" to describe a high level of sociality with an emphasis on personal relationships, in contrast to a "cold", process-orientated work environment, as typically found in an MNC. While the fixed office hours of public organisations were contrasted with Advice Company's flexible work hours in a positive and client-centric connotation, another view was rather critical, relating to forced unpaid overtime work. The differing rating of unfixed work times expresses the organisation's discourse on the opaque meaning of client centricity and the differently perceived consequences of this orientation. These contradicting opinions led the employees to select different reference points for their ideas of "Indianness". In one case, Advice Company was not contrasted to a government office but to another MNC in the private sector economy; in this comparison, Advice Company was presented as more "Indian". Similarly, other colleagues compared Advice Company to government offices or to other MNCs.

Advice Company's "Indianness" is therefore relative to the point of reference selected by each interlocutor: compared with a public sector bank, the practice of addressing colleagues by the first name makes Advice Company less "Indian"; reversely, an MNC with an independent work orienta- 
tion between managers and mentees positions Advice Company as more "Indian". The statements localise experienced or (stereotypically) inferred organisational frameworks in relation to perceptions of Advice Company. This leads me to conclude that a shared notion of "Indianness" can be assumed to exist. However, at Advice Company, my interlocutors saw stronger or weaker traits of "Indianness" in the organisation, depending on their chosen point of reference. This assertion leads me back to my focus on communication in the organisational analysis.

\subsection{Advice Company as a client-centric social system}

Part I (chapters 4-6) showed that Advice Company comprises a social system with both clients and freelancers in its differentiated environment. Clients and freelancers play a significant role in constituting and maintaining the organisational boundaries: interactions with each of these systems in the environment are managed by functions that specialise in boundary work. Advice Company controls this interactional openness through information selection, dedicated transmission formats and differentiated locations. While the interaction with clients and freelancers is structurally similar, the two types of interaction play complementary roles for the organisation. Clients provide the monetary resources by which the organisation is maintained. Through their orders and the subsequent revenues, Advice Company is able to maintain its organisational activity, which includes hiring freelancers for work tasks. As the initial impulse for a project originates from clients, clients are of upmost importance to the organisation. The freelancers receive monetary compensation and therefore occupy the other end of the perceived hierarchy scale. This is reflected in the location of the interaction: while clients are received in the main office's meeting rooms, which feature tea, coffee and biscuits, the freelancer zone in the street office features plastic chairs and lacks air conditioning.

Advice Company's internal differentiation is consequently structured according to the value client centricity (Figure 28). Differences between the three offices in size, access procedures and equipment mirror the functions they host in relation to distance to the client. Consultants, who directly interact with clients, not only fulfil the most prestigious job in the organisation, but they are also located in the sleek, spacious and "corporate" main office next to the organisation's top managers. In contrast, employees who deal directly 
Figure 28: Client centricity scale

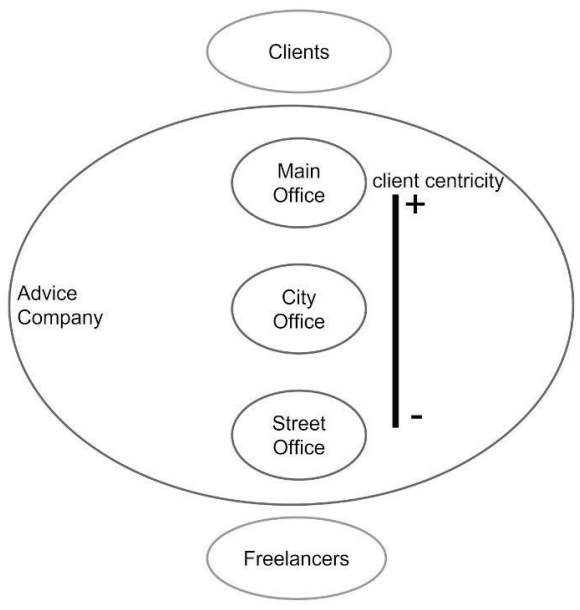

with the freelancers refer to themselves as the "simple people". They work in the "suburban", windowless and sparsely equipped street office with the freelancer's zone upstairs. The city office depicts an intermediary position on the scale, with its light ceiling and decent facilities as the workplace of those whose function is to support client consultants across the world, including those from the main office.

Furthermore, the internal role differentiation and job status on the micro-level, independent of formal management hierarchies (as demonstrated in the relationship between consultants and project managers in the main office) is also aligned with the value client centricity. This status differentiation repeats itself in the city office between the standard and embedded teams: although the job tasks are similar (if not identical), the former teams maintain a lower status due to their lack of direct attachment to an overseas consultant manager and hence greater distance to the client than their peers on the embedded teams.

Even in spatial terms, client centricity is the metaphorically more valued paradigm: when briefing projects and tasks, clients provide "downloads" to consulting teams, who give "downloads" to project coordinators. From their desks on the sixth floor of the main office, client consultants therefore trigger a proverbial downwards slope to communicate with the "ground reality" - 
the opposite of client centricity in a state of hierarchical opposition (sensu Dumont, 1980 [1966]).

As I showed in Part II, the value client centricity not only steers the organisational structure, but also shapes the working patterns around client projects. Both project planning processes and mechanisms of collaboration are based on notions of client centricity, with employees who are closest to the client making a conscious selection of information about the client project for subsequent teams. The relevance of client centricity for the organisation is thus apparently so high that its self-determined structures are geared up to select stimuli from the environment according to this guiding difference, and internal sub-systems repeat this selection process in the same way.

\subsection{Guiding difference as working misunderstandings}

\subsubsection{The opacity of client centricity}

In spite of its relevance for Advice Company, client centricity remains a value of surprising opacity. For example, exactly how far an employee's client-centric attitude should go is unclear to the employees. The point at which client centricity should end is - in every team and situation - subject to negotiation. Should it end at $11.00 \mathrm{pm}$, or when a client request is perceived as utterly senseless? When an employee is in bed with a feverish cold? Or when a client treats an employee disrespectfully over the phone? In attempting to grasp client centricity I have shown an array of its representations, such as the awards (displayed on desks) granted by clients or Advice Company for outstanding performance and the corresponding narratives of my interlocutors' most remarkable projects. The city office employees' eagerness to give meaning to their countless hours of presentation slides formatting through their "analysis" work and their desire to pursue a career path to a client consulting role or to "move to the client side" are other relevant examples.

Similarly, the main office - located on the sixth floor of a building with large glass windows providing stunning views over the area - is the client interaction hub and, with its "corporate" atmosphere, represents client centricity. This location is nevertheless characterised by contradicting notions of distraction, success and fear. These contradictions, the different representations and employees' constant negotiations illustrate that client centricity remains a perpetual working misunderstanding - a value that allows all inter- 
acting parties to attach their own meanings. This enables the organisational system to continue its operations without encountering dissonance across its sub-systems. If Advice Company were to attempt to define client centricity, it would most likely not be able to accommodate the broad set of understandings under which this highly differentiated complex organisation operates. The working misunderstanding of client centricity is not an obstacle, but one of the conditions of successful organisational functioning.

\subsubsection{Ground reality as a corrective limit}

The organisation's dominant value client centricity with its different, contextrelated meanings is counter-balanced by an opposing value which is less explicitly expressed and similarly opaque. As the second value of the organisation's guiding difference it is perceived as a force running against client centricity which is subordinated and therefore not explicitly named. I have decided to call this value ground reality as it is a term used by my interlocutors in the context of causing irritations to the client centric work processes. I have subsumed all the different notions of opposition to client centricity in the value ground reality, which has a different meaning depending on the context.

Ground reality finds its most seizable manifestation in the street office the bare, functional and slightly worn location on a side street of a "rather suburban area", as an employee described it. Ground reality furthermore finds indirect representation at the main office through the specialised function of project coordinators, who manage the translation from client-centric subsystems to the execution teams. The ground reality is the predominant cause of issues and escalations on client projects - the most prevalent examples of unmet client centricity in the organisation. Such issues might be due to the execution teams, or due to their freelancers, who may cause a delay by not delivering on time or not performing tasks to the required quality. At the same time, the ground reality serves to correct client expectations and delineates the boundary of client centricity. With these representations, it becomes apparent that ground reality is a working misunderstanding that allows for parallel encoding. The representations further suggest that ground reality is incompatible with client centricity, as shown in the division of formatting tasks from "analysis" in the city office.

But the ground reality is also represented in the data produced by the freelancers, which constitute the basis of a successful client project. In Ad- 
vice Company's operationally closed organisational system, these data outputs cannot enter directly. Rather, they are the result of mediation that is enabled by the execution teams, who operate according to the value ground reality. This sub-system is necessary for the freelancers' outputs to be utilised for further organisation-maintaining operations. As suggested by the practice of carefully managed client visits at the street office, a client project requires the ground reality for successful completion. In this stage, the relation of the values is reversed, the hierarchy inverted: ground reality is more relevant than client centricity.

\subsection{Mutually exclusive values}

The existence of these two opposing values becomes salient in the organisation's internal differentiation. The necessity of both values for the organisational structure (rather than simply client centricity) can be derived from the fact that differentiation along the client centricity scale is valid in one moment, but reversed in another. Furthermore, Advice Company acknowledges the existence of these two values, as there are specific functions in place to manage the translation between them. The project coordinators manage this translation between client centricity and ground reality, while the city office teams play a central role in making the ground reality (i.e. information from the environment) accessible to client-centric sub-systems by converting it with tremendous effort - into easy-to-use presentations.

These functions must manage the fundamental (and contradictory) incompatibility of the two values. This notion is subsumed in the following quote of project coordinator Nidhi when discussing the challenge of mediating between client consultants and execution teams:

Some of the consultants are like puppets to the client, like: "Ok, you want this thing", "Ok, ok, we'll give this thing" and then they'll start pressurising the other teams. This is not how work should happen. Then you are running out of quality inputs [from the execution teams] and our reputation will go down if we are not working as per the quality. [...] A major challenge is to balance these two kinds of people [the consultants and the execution teams]. The project coordinator's role is to balance these two departments, but it's a real challenge-it's a real challenge. You can't keep both of them happy and keep both of them unhappy. 
The first part of Nidhi's quote relates to the issue of overstepping the organisational boundary. If client consultants get so close to the client that they become part of the environment, no further translation can occur between the client (i.e. the environment) and the organisation. Her remark on the loss of quality positions the ground reality as a corrective to client centricity, though one that is actualised over the long term, given the loss of reputation. In the second part of her quote, Nidhi addresses the mutual exclusiveness of the two values, characterising the conflicting nature of her translation work with no options (in her opinion) for a solution. Remarkable is her standpoint which renders it as unachievable to operate according to both values and thus impossible to select her communications in a way that makes both client consultants and execution team leads "happy". An easy option would most likely be for her to take a client-centric standpoint by prioritising the client consulting team's needs and pressuring the execution teams. Yet with her assertion that both departments cannot be kept unhappy, she acknowledges the relevance of the ground reality for organisational functioning and therefore the need to invert the values, at times (Figure 29). This is in the sense of Dumont the effect of "hierarchical encompassing": "One observes that every time a notion gains importance it acquires the capacity to encompass its contrary" (Dumont 1980 [1966]: 244-245).

Figure 29: Model of the guiding difference

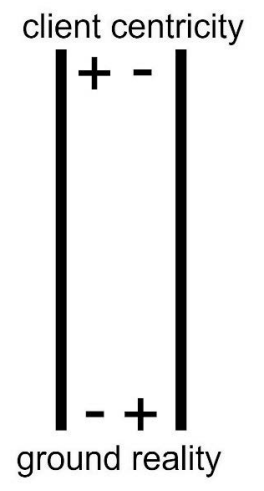




\subsection{Closing the black box}

In this work, I have opened the black box of organisational functioning to untangle, on a micro-level, the mechanisms and selection processes that shape Advice Company's structure and interactions. The analysis of this complex organisation has addressed two research questions: First, I have illustrated how the value client centricity shapes the organisation into a client-centric structure by leveraging opacity to effect a working misunderstanding. Analogous to this is the organisation's differentiation along the opposing yet similarly amorphous value ground reality. This value enables the emergence of further internal differentiations that resist the client-centric structure but are of vital relevance for the organisation's existence. Second, I have illustrated the productive role of both intentional and unintentional working misunderstandings for the organisation's operations, drawing on the example of the client project. I have shown that a client project must operate as a working misunderstanding in order to allow for meaningful selections of understanding and processing in the various organisational sub-systems.

Answering these two research questions, however, has led to an additional conclusion: the black box must remain closed. This detailed, micro-level insight into the black box of organisational functioning has revealed different areas of working misunderstandings that enable successful complex operations. I have also shown that these operations rely on the opacity between systems and the ambiguities associated with the two values of the guiding difference. When the black box is closed, the system works - and works very well: Advice Company is highly successful in the industry; the organisation has an excellent reputation in its field and its advice is valued by clients across the globe.

The aim of full transparency in organisational functioning and collaboration processes is an understandable assumption of normative management theories such as transaction cost economics (Acheson 2002) and (economic) value chain models of organisations (Porter 2001). Bringing working misunderstandings to a "point of unravelling" (Reed 2006) by enabling deeper insight into the selection processes of other sub-systems might be desired by management boards in an attempt to maintain the "dream of rationality" behind organisational decision-making processes (Brunsson 2006: 13). But the organisational system requires opacity - as represented by the black box - in order to function. 
I have shown that working misunderstandings are part and parcel of the interactions in complex organisations. They emerge in conjunction with system differentiation and provide the mechanism for maintaining boundaries between sub-systems while allowing for interactional openness. I have thus shown how and why working misunderstandings arise and why they are productive in the context of complex organisations.

While these insights might allow for a set of resolution models or checklists to ward off misunderstandings, the key conclusion from my work is that it would be unproductive for organisations to attempt to defeat them. Accordingly, the Indo-German project incident from my own experience, as presented at the beginning of this work, appears in a different light. Maybe we should have relied more on the opacities between the two systems - the black box - and the working misunderstandings, rather than bringing communication to a sudden end by escalating it. Hence, the answer to my ex-colleague's question of how I could resolve the issues around collaboration might have been to continue communicating and accept that the Indian offshore-team was opaque to us and that both we and they had to operate according to different understandings of the project. Such an answer is not normative, but it follows the approach of the descriptive discipline of organisational anthropology.

This ethnography, which was motivated by my own curiosity and interest in the functioning of complex organisations, has aimed at producing scientific insights. In this case, the insights - as generated from a detailed view into the black box - have led to the informed conclusion that to maintain system functioning, we should close the box again.

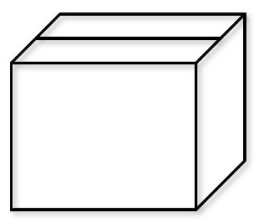




\section{Acknowledgments}

Many people and institutions provided help and support for this project, from the initial idea to my fieldwork in India, to the writing of this monograph. I would like to use this opportunity to express my gratitude for their help, here.

The generous candidness of the organisation's CEO to open the doors of his offices for me provided me with a rare opportunity that I cannot value highly enough. This CEO will need to remain as anonymous as the organisation he led, but I am grateful for his trust in me and my project. Similarly, his $\mathrm{HR}$ director was of a congenial understanding with respect to my $\mathrm{PhD}$ research and endowed me with full freedom for my fieldwork. But the frame would have remained empty without the many employees who allowed me to accompany them and who patiently answered my numerous questions many of whom even prolonged their workday for our interviews. After switching off their computers at the end of the day, some lavishly showed me their after-office life by taking me along to their favourite coffee shops and opening their homes for me on festival weekends with their families and Sunday chillouts with their friends. Via social media, I am still in loose touch with many, in closer touch with a few and have even met a handful of my former interlocutors in Europe. I would like to thank all of them for their openness and support. Many thanks go to my flatmates Nidhi and Tania, who facilitated the easiest start into a new setting I could have wished for, and who became good friends in the 11 months we shared "our home".

My primary supervisor Prof. Dr. Guido Sprenger endorsed my project from the very beginning and his regular doses of advice were milestones that motivated me each time anew. I cannot thank him enough for his enthusiasm, intellectual engagement and time spent reading my work and talking about it. I thank Prof. Dr. Michael Schönhuth for his continuous interest in my project and for his open feedback whenever I approached him with questions. 
I thank Dr. Christian Strümpell for the numerous pieces of advice he gave me during the preparation phase before I left for fieldwork, which proved invaluable. I am grateful for the support of Dr. Susanne Spülbeck, who devoted many hours to Skype, supervising me regularly throughout my year of fieldwork. Her focused questions and comments helped me shape the research phase and circumnavigate potentially difficult situations. I thank the German Academic Exchange Service (DAAD) for the scholarship funding of the fieldwork phase in India.

I thank the Heidelberg Graduate School for the Humanities and Social Sciences (HGGS) for the opportunity to exchange thoughts with fellow PhD students across different faculties in an appreciative interdisciplinary environment throughout my three years in the structural doctoral programme. Many thanks are due to my fellow PhD students at the Institute of Anthropology for their intellectual companionship in our doctoral colloquiums and beyond. Special thanks go to Carmen Grimm for cross-reading our draft texts and organising critical feedback sessions about them.

I would also like to thank Dr. Franziska Herbst and Dr. Anne Bastian for their friendship and the many motivating, cheerful and reflexive conversations we had about this dissertation. I am particularly grateful for the unlimited support of my parents for my project, and also for taking me away from it - whether from my fieldwork or my desk at home - for short periods of time. Finally, for her patience, understanding, encouragement, intelligent humour and love throughout the entire dissertation phase, I thank Lena. 


\section{List of Figures}

Figure 1: Fieldwork phases and locations, p.57.

Figure 2: Chapter outline, Part I, p.77.

Figure 3: Map of entry procedures to the main office, p. 87.

Figure 4: Structural similarities: Clients vs. freelancers, p.105.

Figure 5: Communication density in the main office, p.137.

Figure 6: Client centricity scale, p.151.

Figure 7: The guiding difference client centricity/ground reality, p.152.

Figure 8: Communication patterns of client consultants, p.158.

Figure 9: Communication patterns of project coordinators, p.158.

Figure 10: City office team map, p.163.

Figure 11: Reversed values at the street office, p.166.

Figure 12: Lunch group connections at the city office, p.168.

Figure 13: L/M quadrant and chapter outline of Part II, p.176.

Figure 14: The L/M quandrant of working misunderstandings, p.186.

Figure 15: The project development process, p.188.

Figure 16: Project process and offices, p.189.

Figure 17: Chapter allocation on the L/M quadrant, p.191.

Figure 18: Chapter 9 on the L/M quadrant, p.201.

Figure 19: Project Phase and Information levels, p.212.

Figure 20: Chapter 10 position on the L/M quadrant, p.227.

Figure 21: Allocation of Section 10.2. in the project process, p.233.

Figure 22: Section 10.3 in the project process, p.238.

Figure 23: Allocation of Section 10.4 in the project process, p.247.

Figure 24: Allocation of Section 10.5 in the project process, p.259.

Figure 25: Balancing tasks at the city office, p. 265.

Figure 26: Allocation of Section 10.6 in the project process, p.271.

Figure 27: Part II categories of working misunderstandings, p.279. 
296 Working Misunderstandings

Figure 28: Client centricity scale, p.286.

Figure 29: Model of the guiding difference, p.290. 


\section{References}

Acheson, James (2002): Transaction Cost Economics: Accomplishments, Problems, and Possibilities, in Ensminger, Jean (ed.): Theory in economic anthropology. Walnut Creek: AltaMira Press, 27-58.

Alvesson, Mats (1995): Management of knowledge intensive companies. Berlin: de Gruyter.

Amit, Vered (2000): Introduction: Constructing the Field, in Amit, Vered (ed.): Constructing the field: ethnographic fieldwork in the contemporary world. London: Routledge, 1-18.

Appadurai, Arjun (1988): The social life of things: commodities in cultural perspective. Cambridge: Cambridge University Press.

Appadurai, Arjun (2008): Modernity at large: cultural dimensions of globalization. Minneapolis: University of Minnesota Press.

Augustynek, Marta (2010): Arbeitskulturen im Großkonzern: eine kulturanthropologische Analyse organisatorischer Transformationsdynamik in Mitarbeiterperspektive. Münster: Waxmann.

Bachmann, Götz (2014): Kollegialität: Eine Ethnografie der Belegschaftskultur im Kaufhaus. Frankfurt: Campus Verlag.

Baba, Marietta (2006): Anthropology and business, in Birx, James (ed.): Encyclopedia of anthropology. Thousand Oaks: SAGE, 84-117.

Baer, Drake (2014): Here's Why Companies Are Desperate To Hire Anthropologists. Business Insider, 27.3.2014. URL: http://www.businessinsider.co $\mathrm{m} /$ heres-why-companies-aredesperateto-hireanthropologists-2014-3?IR $=\mathrm{T}$ [last accessed 18.05.2021]

Banerjee, Abhradip, Das, Arnab, and Chakrabarti, Gopalkrishna (2011): Tricks and Truths of Drug Marketing: An Insider's Experience of an Indian Pharmaceutical Organization. Asian Journal of Social Science, 39 (5), 581-604. 
Barab, Sasha, Thomas, Michael, Dodge, Tyler, Squire, Kurt, and Newell, Markeda (2004): Critical Design Ethnography: Designing for Change. Anthropology \& Education Quarterly, 35 (2), 254-68.

Barley, Stephen and Kunda, Gideon (2004): Gurus, hired guns, and warm bodies: Itinerant experts in a knowledge economy. Princeton: Princeton University Press.

Baskerville, Rachel (2003): Hofstede never studied culture. Accounting, Organizations and Society, 28 (1), 1-14.

Baskerville, Richard and Myers, Michael (2015): Design ethnography in information systems. Information Systems Journal, 25 (1), 23-46.

Bateson, Gregory (1972): Steps to an ecology of mind: Collected essays in anthropology, psychiatry, evolution, and epistemology. New York: Ballantine.

Batteau, Allen (2000): Negations and Ambiguities in the Cultures of Organization. American Anthropologist, 102 (4), 726-40.

Baviskar, Amita and Ray, Raka (eds.) (2011): Elite and everyman: the cultural politics of the Indian middle classes. London: Routledge.

Bayer, Julia (2013): Media Diversity in Deutschland. Kulturwissenschaftliche Perspektiven auf journalistische Praxis. München: Ludwigs-MaximiliansUniversität Institut für Ethnologie Dissertationen. URL: https://edoc.ub. uni-muenchen.de/16086/1/Bayer_Julia.pdf [last accessed 18.05.2021]

Beech, Nic (2011): Liminality and the practices of identity reconstruction. Human Relations, 64 (2), 285-302.

Bernard, Harvey (2006): Research methods in anthropology: qualitative and quantitative approaches. Lanham: AltaMira Press.

Biao, Xiang (2007): Global "body shopping" : an Indian labor system in the information technology industry. Princeton: Princeton University Press.

Bohannan, Paul (1964): Africa and Africans. New York: The Natural History Press.

Böhme, Gernot (1995): Atmosphäre: Essays zur neuen Ästhetik. Frankfurt am Main: Suhrkamp.

Böhme, Gernot (2001): Aisthetik: Vorlesungen über Ästhetik als allgemeine Wahrnehmungslehre. München: Fink.

Böhme, Gernot (2013): Architektur und Atmosphäre. München: Fink.

Borg, Elisabeth and Söderlund, Jonas (2013): Moving in, moving on: liminality practices in project-based work. Employee Relations, 36 (2), 182-97.

Borgatti, Stephen and Everett, Martin (2000): Models of core/periphery structures. Social Networks, 21 (4), 375-95. 
Brandhoff, Boris (2009): Autopoietic Systems, Not Corporate Actors: A Sketch of Niklas Luhmann's Theory of Organisations. European Business Organization Law Review, 10 (2), 307-22.

Breidenbach, Joana and Nyíri, Pál (2001): Kulturelle Kompetenz als Business. Organisationsentwicklung, 4 (1), 70-77.

Breidenbach, Joana and Nyíri, Pál (2009): Seeing culture everywhere: From genocide to consumer habits. Seattle: University of Washington Press.

Brosius, Christiane (2010): India's middle class: new forms of urban leisure, consumption and prosperity.London: Routledge.

Brunsson, Nils (2006): Mechanisms of hope: maintaining the dream of the rational organization. Herndon: Copenhagen Business School Press.

Callon, Michel (1986): Some elements of a sociology of translation: domestication of the scallops and the fishermen of St Brieuc Bay. The Sociological Review, 32 (1), 196-233.

Cattani, Gino and Ferriani, Simone (2008): A Core/Periphery Perspective on Individual Creative Performance: Social Networks and Cinematic Achievements in the Hollywood Film Industry. Organization Science, 19 (6), 824-44.

Cayla, Julien, Beers, Robin, and Arnould, Eric (2014): Stories that deliver business insights, MIT Sloan Management Review, 55 (2), 55-62. URL: http:/ /sloanreview.mit.edu/article/stories-that-deliver-business-insights/ [last accessed 18.05.2021]

Cefkin, Melissa (2010a): Introduction, in Cefkin, Melissa (ed.): Ethnography and the corporate encounter: reflections on research in and of corporations New York: Berghahn, 1-40.

Cefkin, Melissa (ed.) (2010): Ethnography and the corporate encounter: reflections on research in and of corporations New York: Berghahn.

Chen-Wishart, Mindy (2013): Legal Transplant and Undue Influence: Lost in Translation or a Working Misunderstanding?. International and Comparative Law Quarterly, 62 (1), 1-30.

Chhokar, J., Brodbeck, F., and House, R. (eds.) (2008): Culture and leadership across the world: the GLOBE book of in-depth studies of 25 societies. Mahwah: Erlbaum.

Cole, Jennifer (2014): Working Mis/Understandings: The Tangled Relationship between Kinship, Franco-Malagasy Binational Marriages, and the French State. Cultural Anthropology, 29 (3), 527-51.

Czarniawska, Barbara (1997): Narrating the organization: dramas of institutional identity. Chicago: University of Chicago Press. 
Czarniawska, Barbara and Mazza, Carmelo (2003): Consulting as a Liminal Space. Human Relations, 56 (3), 267-90.

Czarniawska, Barbara (2007): Shadowing and other techniques for doing fieldwork in modern societies. Malmö: Liber.

Dahlén, Tommy (1997): Among the interculturalists: An emergent profession and its packaging of knowledge. Stockholm: Stockholm University Department of Social Anthropology Dissertations. URL: http://su.diva-porta l.org/smash/get/diva2:377715/FULLTEXTo1.pdf [last accessed 20.05.2021]

Dalsgaard, Steffen (2013): The field as a temporal entity and the challenges of the contemporary. Social Anthropology, 21 (2), 213-25.

Dalsgaard, Steffen and Nielsen, Morten (2013): Introduction: time and the field. Social Analysis, 57 (1), 1-19.

Davala, Sarath (1995): Labour strategies of multinational corporations in India. New Delhi: Friedrich-Ebert-Stiftung.

Davenport, Tom (2007): 'The rise of corporate anthropology', Harvard Business Review, 28, 1-2. URL: https://hbr.org/2007/11/the-rise-of-corporateanthropo [last accessed 18.05.2021]

De Neve, Geert (2009): Asking for giving Baki: Neo-bondage, or the Interplay of Bondage and Resistance in the Tamilnadu Power-loom Industry, in Mollona, Massimiliano, De Neve, Geert, and Parry, Jonathan (eds.): Industrial Work and Life: An Anthropological Reader Oxford: Berg, 363-86.

Deery, Margaret and Jago, Leo K. (2002): The core and the periphery: an examination of the flexible workforce model in the hotel industry. International Journal of Hospitality Management, 21 (4), 339-51.

Denny, Rita and Sunderland, Patricia (eds.) (2014): Handbook of anthropology in business. Walnut Creek: Left Coast Press.

Desai, Malavika, Majumdar, Bishakha, Chakraborty, Tanusree and Ghosh, Kamalika. (2011): The second shift: working women in India. Gender in Management, 26 (6), 432-50.

Diel-Khalil, Helga and Götz, Klaus (1999): Ethnologie und Organisationsentwicklung. München: Hampp.

Dirksen, Vanessa, Huizing, Ard, and Smit, Bas (2010): 'Piling on layers of understanding: the use of connective ethnography for the study of (online) work practices. New Media \& Society, 12 (7), 1045-63.

Dorward, David (1974): Ethnography and Administration: A Study of Anglo-Tiv 'Working Misunderstanding'. The Journal of African History, 15 (3), 457-77.

Dumont, Louis (1980 [1966]): Homo hierarchicus: the caste system and its implications. Chicago: University of Chicago Press. 
Dumont, Louis (2013): On value: The Radcliffe-Brown Lecture in Social Anthropology, 1980, HAU: Journal of Ethnographic Theory, 3 (1), 287-315.

Durrenberger, Paul (1975): Understanding a Misunderstanding: Thai-Lisu Relations in Northern Thailand. Anthropological Quarterly, 48 (2), 106-20.

Eaton, Tara (2011): A cultural analysis of information technology offshore outsourcing: an exercise in multi-sited ethnography of virtual work. Detroit: Wayne State University Dissertations. URL: http://digitalcommons.wayne .edu/cgi/viewcontent.cgi?article $=1369 \&$ context=oa_dissertations [last accessed 18.05.2021]

Egger, Simone (2015): Stadt, Ästhetik und Atmosphäre. Dimensionen der Wahrnehmung im urbanen Raum, in Irene Götz, Moser, Johannes, Ege, Moritz und Lauterbach, Burkhard (eds.): Europäische Ethnologie in München: ein kulturwissenschaftlicher Reader Münster: Waxmann, 13766.

Eriksen, Thomas H. (2006): Engaging anthropology: the case for a public presence Oxford: Berg.

Eriksen, Thomas H. (2007): Globalization: The Key Concepts. Oxford: Berg.

Fabian, Johannes (1995): Ethnographic misunderstanding and the perils of context. American Anthropologist, 97 (1), 41-50.

Färber, Alexa (2006): Weltausstellung als Wissensmodus: Ethnographie einer Repräsentationsarbeit. Münster: LitVerlag.

Faßauer, Gabriele and Geithner, Silke (2016): Entgrenzung und Grenzarbeit in Co-Konfiguration: Eine tätigkeitstheoretische Perspektive. Industrielle Beziehungen, 23 (2), 92-112.

Fine, Gary (2009): Kitchens: The Culture of Restaurant Work. Berkeley: University of California Press.

Freeman, Carla (2009): Femininity and Flexible Labour: Fashioning Class Through Gender on the Global Assembly Line, in Mollona, Massimiliano, De Neve, Geert, and Parry, Jonathan (eds.): Industrial Work and Life: An Anthropological Reader, Oxford: Berg, 257-70.

Frohnen, Anja (2005): Diversity in action: Multinationalität in globalen Unternehmen am Beispiel Ford. Bielefeld: Transcript.

Gadamer, Hans-Georg (2010 [1960]): Gesammelte Werke: Hermeneutik : Wahrheit und Methode. - Grundzüge einer philosophischen Hermeneutik. Bd. 1. Tübingen: Mohr Siebeck.

Gadamer, Hans-Georg (2004): Truth and Method. Translation by J. Weinsheimer and D. Marshall (Wahrheit und Methode). London: Continuum. 
Gamst, Frederick and Helmers, Sabine (1991): Die kulturelle Perspektive und die Arbeit: Ein forschungsgeschichtliches Panorama der nordamerikanischen Industrieethnologie. Zeitschrift für Ethnologie, 116, 25-41.

Garg, Arun Kumar (1992): Multinational corporations in India: export performance and promises. Meerut: Friends.

Garsten, Christina (1994): Apple World: Core and Periphery in a Transnational Organizational Culture: A Study of Apple Computer Inc. Philadelphia: Coronet Books.

Garsten, Christina (1999): Betwixt and between: temporary employees as liminal subjects in flexible organizations. Organization studies, 20 (4), 601-17.

Garsten, Christina and Nyqvist, Anette (2013): Entries: Engaging ôrganisational worlds, in Garsten, Christina and Nyqvist, Anette (eds.): Organisational anthropology: doing ethnography in and among complex organisations London: Pluto, 1-28.

Gavin, Diane (2015): Organizational Ethnographic Research: Intersections of Place, Cultural Identities, Constructed Memories, and Sense Making Within Institutions. Journal of Psychological Issues in Organizational Culture, 6 (2), 95-100.

Gellner, David and Hirsch, Eric (eds.) (2001): Inside organizations: anthropologists at work. Oxford: Berg.

Gershon, Ilana (2005): Seeing like a system. Anthropological Theory, 5 (2), 99116.

Gjerde, Per (2004): Culture, Power, and Experience: Toward a Person-Centered Cultural Psychology. Human Development, 47 (3), 138-57.

Glauser, Laura (2016): Das Projekt des unternehmerischen Selbst. Eine Feldforschung in der Coachingzone. Bielefeld: transcript.

Goffman, Erving (1959): The presentation of self in everyday life. New York: Doubleday.

Gunn, Wendy, Otto, Tom, and Smith, Rachel Charlotte (eds.) (2013): Design anthropology: theory and practice. London: Bloomsbury.

Gupte, Niteen and Müller-Gupte, Anke (2010): Indischer Arbeitsplatz aus deutscher Sicht, in Casper-Hehne, Hiltraud, Gupte, Niteen and Stilz, Gerhard (eds.): Kommunikation über Grenzen: aktuelle Ansätze zur interkulturellen Verständigung Göttingen: Universitätsverlag Göttingen, 159-90.

Hampden-Turner, Charles and Trompenaars, Fons (1997): Response to Geert Hofstede. International Journal of Intercultural Relations, 21 (1), 149-59. Hannerz, Ulf (2003a): Being there... and there... and there!: Reflections on Multi-Site Ethnography. Ethnography, 4 (2), 201-16. 
Hannerz, Ulf (2003b): Transnational connections: culture, people, places. London: Routledge.

Hannerz, Ulf (2006): Studying Down, Up, Sideways, Through, Backwards, Forwards, Anway and at Home: Reflections on the Field Worries of an Expansive Discipline, in Coleman, Simon and Collins, Peter (eds.): Locating the Field: Space, Place and Context in Anthropology Oxford: Berg, 23-43.

Hanson, Nathalie (2014): Recognizing Agile in Denny, Rita and Sunderland, Patricia (eds.): Handbook of anthropology in business (Walnut Creek: Left Coast Press), 379-95.

Hawkins, Beverley (2008): Double Agents: Gendered Organizational Culture, Control and Resistance. Sociology, 42 (3), 418-35.

Heerwagen, Judith, Kampschroer, Kevin, Powell, Kevin, and Loftness, Vivian (2004): Collaborative knowledge work environments. Building Research \& Information, 32 (6), 510-28.

Heidemann, Frank (2018): Dorfatmosphären. Hatti und keri der Badaga in Südindien, Paideuma - Mitteilungen zur Kulturkunde, 64, 51-73.

Hofstede, Geert (1996): Riding the waves of commerce: A test of trompenaars'"model" of national culture differences. International Journal of Intercultural Relations, 20 (2), 189-98.

Hofstede, Geert, Hofstede, Gert Jan, and Minkov, Michael (2010): Cultures and organizations : software of the mind; intercultural cooperation and its importance for survival. New York: McGraw-Hill.

Holtgrewe, Ursula (2003): Gute und schöne Dienstleistung. Gestaltung der Grenzstellen und der Kunden, in Jacobsen, Heike and Voswinkel, Stephan (eds.): Dienstleistungsarbeit - Dienstleistungskultur: Beiträge zu einer Fachtagung am Landesinstitut Sozialforschungsstelle Dortmund 2003. SAMF Arbeitspapier 2003-1 Dortmund: Landesinstitut Sozialforschungsstelle, 65-76.

Holzer, Boris (2010): Netzwerke und Systeme. Zumverhältnis von Vernetzung und Differenzierung, in Stegbauer, Christian (ed.): Netzwerkanalyse und Netzwerktheorie : Ein neues Paradigma in den Sozialwissenschaften Wiesbaden: VS Verlag für Sozialwissenschaften, 155-64.

Hopgood, Stephen (2006): Keepers of the flame: understanding Amnesty International. London: Cornell University Press.

House, Robert, Hanges, Paul, Javidan, Mansour, Dorfman, Peter, and Gupta, Vipin (2004): Culture, leadership, and organizations: The GLOBE study of 62 societies. Thousand Oaks: Sage. 
Huber, Birgit (2012): Arbeiten in der Kreativindustrie: eine multilokale Ethnografie der Entgrenzung von Arbeits- und Lebenswelt. Frankfurt am Main: Campus.

Hüsken, Thomas (2006): Der Stamm der Experten: Rhetorik und Praxis des Interkulturellen Managements in der deutschen staatlichen Entwicklungszusammenarbeit. Bielefeld: transcript.

Iliffe, John (1979): A modern history of Tanganyika. Cambridge: Cambdridge University Press.

Jaffee, Daniel (2012): Weak Coffee: Certification and Co-Optation in the Fair Trade Movement. Social Problems, 59 (1), 94-116.

Jiménez, Alberto Corsin (ed.) (2016 [2007]): The Anthropology of Organisations. New York: Routledge.

Johri, Lalit (1983): Business strategies of multinational corporations in India : case study of drug and pharmaceutical industry. New Delhi: Vision Books.

Jones, Michael (2007): Hofstede- Culturally Questionable? Paper presented at the Oxford Business \& Economics Conference, 24-26 June, 2007. Oxford.

Jones, Michael, Moore, Michael, and Snyder, Richard (eds.) (1988): Inside organizations: understanding the human dimension. Newbury Park: Sage. Jordan, Ann (2003): Business anthropology. Long Grove: Waveland Press.

Jordan, Brigitte and Dalal, Brinda (2006): Persuasive Encounters: Ethnography in the Corporation. Field Methods, 18 (4), 359-81.

Kapferer, Bruce (2011): Louis Dumont and a Holist Anthropology. In Otto, Ton; and Nils Bubandt (eds), Experiments in Holism: Theory and Practice in Contemporary Anthropology, London: Wiley, 187-208.

Keiding, Tina (2010): Observing Participating Observation-A Re-description Based on Systems Theory. Forum Qualitative Sozialforschung, 11 (3). URL: http://www.qualitative-research.net/index.php/fqs/article/view/153 8/3041 [last accessed 21.11.2016]

Khandelwal, Kanika (2009): In search of Indian-ness: cultures of multinationals. New Delhi: Kanishka Publishers.

Kiepenheuer-Drechsler, Barbara (2013): Vielfalt plus Zusammenhalt: eine ethnologische Perspektive auf die Praxis Berliner Integrationspolitik. Bielefeld: transcript.

Kitner, Kathleen (2014): The good Anthropologist: Questioning Ethics in the Workplace. In Denny, Rita and Sunderland, Patricia (eds.): Handbook of anthropology in business Walnut Creek CA: Left Coast Press, 309-20. 
Kleiner, Art (2001): The Dilemma Doctors. strategy+business, 23. URL: $h$ ttp://www.strategy-business.com/article/17251?gko $=444 \mathrm{C1}$ [last accessed 18.05.2021]

Klepic, Jure (2014): Applying Anthropology Concepts to Business Models. Huffington Post, 21.09.2014. URL: http://www.huffingtonpost.co $\mathrm{m} /$ jure-klepic/applying-anthropology-con_b_5849242.html [last accessed 18.05.2021]

Kogan, Sandra and Muller, Michael (2006): Ethnographic study of collaborative knowledge work. IBM Systems Journal, 45 (4), 759-71.

Kostera, Monika (1997): The Kitsch-Organization. Studies in Cultures, Organizations \& Societies, 3 (2), 163-77.

Krasberg, Ulrike (1998): Kultur als Ethnographie der Grenzüberschreitung, in Giordano, Christian, Colombo Dougoud, Roberta, and Kappus, ElkeNicole (eds.): Interkulturelle Kommunikation im Nationalstaat. Münster: Waxmann, 31-44.

Krause-Jensen, Jakob (2013): Counter-espionage. Fieldwork among culture experts in Bang \& Olufsen, in Garsten, Christina and Nyqvist, Anette (eds.): Organizational Anthropology. Doing Ethnography in and Among Complex Organizations. London: Pluto, 43-57.

Kühl, Stefan (2011): Organisationen: Eine sehr kurze Einführung. Wiesbaden: VS Verlag für Sozialwissenschaften.

La Cecla, Franco (2002): Le malentendu. Paris: Éditions Balland.

Latour, Bruno (1987): Science in action: how to follow scientists and engineers through society. Cambridge: Harvard University Press.

Latour, Bruno (1996): On actor-network theory: A few clarifications. Soziale Welt, 47 (4), 369-81.

Latour, Bruno (2000): Die Hoffnung der Pandora: Untersuchungen zur Wirklichkeit der Wissenschaft. Frankfurt am Main: Suhrkamp.

Lee, Daniel (2007): Observing Communication: Niklas Luhmann and the Problem of Ethnography. Soziale Systeme, 13 (1-2), 456-67.

Li, Yulong, Tarafdar, Monideepa, and Subba, Rao (2012): Collaborative knowledge management practices. International Journal of Operations \& Production Management, 32 (4), 398-422.

Livingston, Julie (2007): Productive Misunderstandings and the Dynamism of Plural Medicine in Mid-Century Bechuanaland. Journal of Southern African Studies, 33 (4), 801-10. 
Lobo, Nancy and Shah, Jayesh (eds.) (2015): The trajectory of India's middle class : economy, ethics and etiquette. Newcastle upon Tyne: Cambridge Scholars Publishing.

Losonczy, Anne-Marie and Mesturini Cappo, Silvia (2014): Ritualized Misunderstanding Between Uncertainty, Agreement, and Rupture. Communication Patterns in Euro-American Ayahuasca Ritual Interactions, in Beatriz Caiuby Labate and Cavnar, Clancy (eds.): Ayahuasca Shamanism in the Amazon and Beyond New York: Oxford University Press, 106-28.URL: http:/www.oxfordscholarship.com/view/10.1093/acp rof:oso/9780199341191.001.0001/acprof-9780199341191-chapter-6 [last accessed 18.05.2021]

Lueders, Christian (2000): Beobachten im Feld, in: Flick, Uwe, von Kardorff, Ernst und Steinke, Ines (eds.): Qualitative Forschung. Ein Handbuch Reinbek: Rowohlt, 384-401.

Luhmann, Niklas (1964): Funktionen und Folgen formaler organisation. Berlin: Duncker \& Humblot.

Luhmann, Niklas (1984): Soziale Systeme: Grundriß einer allgemeinen Theorie. Frankfurt am Main: Suhrkamp.

Luhmann, Niklas (1986): The autopoiesis of social systems, in Geyer, Felix and van der Zouwen, Johannes (eds.): Sociocybernetic paradoxes: observation, control and evolution of self-steering systems London: Sage, 172-92.

Luhmann, Niklas (1988): Closure and openness: On reality in the world of law, in Teubner, Gunther (ed.): Autopoietic law: A new approach to law and society Berlin: De Gruyter, 335-48.

Luhmann, Niklas (1991): Operational Closure and Structural Coupling: The Differentiation of the Legal System. Cardozo Law Review, 13 (5), 1419-42.

Luhmann, Niklas (1995a): Social systems. Translation byJohn Bednarz with Dirk Baecker. Stanford: Stanford University Press.

Luhmann, Niklas (1995b): Soziologische Aufklärung 6: Die Soziologie und der Mensch. Opladen: Westdeutscher Verlag.

Luhmann, Niklas (1997): Die Gesellschaft der Gesellschaft. Frankfurt am Main: Suhrkamp.

Luhmann, Niklas (2006a): Organisation und Entscheidung. Wiesbaden: VS Verl. für Sozialwiss.

Luhmann, Niklas (2006b): System as Difference. Organization, 13 (1), 37-57. Madden, Raymond (2010): Being ethnographic: a guide to the theory and practice of ethnography. Los Angeles: Sage. 
Mahadevan, Jasmin (2009): Standortübergreifende Ingenieursarbeit als interkulturelles Projekt - vom interkulturellen Training zum Cross-Site Consulting, in Koch, Eckart Koch and Speiser, Sabine (eds.): Interkulturelle Projekte - Angewandte Interkulturalität München: Mehring, 10720.

Maitra, Shipra (2008): Dynamics of High-Tech Urban Spaces: Lessons from IT Sector Expansion in the National Capital Region, India, in Ramachandraiah, Chennamsetty, van Westen, A. and Prasad, Sheela (eds.): High-tech urban spaces: Asian and European perspectives New Delhi: Manohar, 25992.

Malinowski, Bronislaw (2005 [1921]): Argonauts of the Western Pacific: an account of native enterprise and adventure in the Archipelagoes of Melanesian New Guinea. London: Routledge.

Jumpertz, Sylvia (2009): Was macht eigentlich ein Organisationsethnologe? ManagerSeminare 141, 56-61. URL: http://www.managerseminare.de/ms _Artikel/Spezialisten-in-Serie-Was-macht-eigentlich-ein-Organisations ethnologe,187273 [last accessed 18.05.2021]

Marcus, George (1995): Ethnography in/of the World System: The Emergence of Multi-Sited Ethnography. Annual Review of Anthropology, 24 (1), 95-117.

Marcus, George and Fischer, Michael (1986): Anthropology as cultural critique: an experimental moment in the human sciences. Chicago: University. of Chicago Press.

Martens, Wil (2006): The distinctions within organizations: Luhmann from a cultural perspective. Organization, 13 (1), 83-108.

Martinussen, John (1988): Transnational corporations in a developing country: the Indian experience. New Delhi: Sage.

Mayer-Ahuja, Nicole (2011a): Grenzen der Homogenisierung: IT-Arbeit zwischen ortsgebundener Regulierung und transnationaler Unternehmensstrategie. Frankfurt am Main: Campus-Verlag.

Mayer-Ahuja, Nicole (2011b): 'I felt like a kid in front of them': Work Organization and Human Life Cycle in Indo-German Software Programming, in Behal, Rana, Fall, Babacar, and Mah, Alice (eds.): Rethinking work: global, historical and sociological perspectives New Delhi: Tulika Books, 9-22.

Mazumdar, Surajit (2012): Industrialization, dirigisme and capitalists: Indian big business from independence to liberalization. New Delhi: Nehru Memorial Museum and Library.

Mazzarella, William (2004): Shoveling smoke: advertising and globalization in contemporary India. New Delhi: Oxford University Press. 
McDonald, Seonaidh (2005): Studying actions in context: a qualitative shadowing method for organizational research. Qualitative Research, 5 (4), 455-73.

McSweeney, Brendan (2002): Hofstede's model of national cultural differences and their consequences: A triumph of faith - a failure of analysis. Human relations, 55 (1), 89-118.

Meyer, Erin (2014): The culture map: Breaking through the invisible boundaries of global business. New York: PublicAffairs.

Mintz, Beth and Schwartz, Michael (1981): Interlocking Directorates and Interest Group Formation. American Sociological Review, 46 (6), 851-69.

Mische, Ann and White, Harrison (1998): Between Conversation and Situation: Public Switching Dynamics across Network Domains. Social Research, 65 (3), 695-724.

Moeran, Brian (2007): A Japanese advertising agency: an anthropology of media and markets. London: Routledge.

Monteiro, Marko and Keating, Elizabeth (2009): Managing misunderstandings: the role of language in interdisciplinary scientific collaboration. Science Communication, 31 (1), 6-28.

Moosmüller, Alois (2007): Interkulturelle Kommunikation aus ethnologischer Sicht, in Moosmüller, Alois (ed.): Interkulturelle Kommunikation: Konturen einer wissenschaftlichen Disziplin Münster: Waxmann, 13-50.

Moosmüller, Alois (2009): Kulturelle Differenz: Diskurse und Kontexte. In Moosmüller, Alois (ed.) Konzepte kultureller Differenz Münster: Waxmann, 13-45.

Moosmüller, Alois and Schönhuth, Michael (2009): Intercultural competence in German discourse. In Deardorf, Darla (ed.): The Sage handbook of intercultural competence Los Angeles: Sage, 209-32.

Mörike, Frauke (2016): Working misunderstandings and notions of collaboration. Towards a framework of working misunderstanding as analytical category for ethnographic insight. Civilisations, 64 (1\&2), 153-68.

Mörike, Frauke (2018): "During a busy day I don't get much done". On the materiality of immaterial labour in a multinational professional services firm. Ethnoscripts, 19(2). https://journals.sub.uni-hamburg.de/ethnoscri pts/article/view/1178 [last accessed 14.05.2021]

Mörike, Frauke and Spülbeck, Susanne (2019): Unternehmenskultur zwischen akademischer Welt und betrieblicher Anwendungspraxis. In KlockeDaffa, Sabine (Hg.), Angewandte Ethnologie - Perspektiven einer anwendungsorientierten Wissenschaft, Wiesbaden: Springer, 537-58. 
Mörike, Frauke (2019): Ethnographic Methods for Human Factors Researchers: Collecting and Interweaving Threads of HCI. In Extended Abstracts of the 2019 CHI Conference on Human Factors in Computing Systems (CHI EA '19). https://doi.org/10.1145/3290607.3298814 [last accessed 15.05.2021]

Morrill, Calvin, Buller, David, Buller, Mary, Larkey, Linda (1999): Toward an Organizational Perspective on Identifying and Managing Formal Gatekeepers. Qualitative Sociology, 22 (1), 51-72.

Murty, Sudarshan (1998): Multinational corporations in India. Jaipur: RBSA Publishers.

Nadége, Chabloz (2007): Le malentendu. Les rencontres paradoxales du 'tourisme solidaire'. Actes de la recherche en sciences sociales 107 (5), 3247.

Nakar-Wallraff, Janaki (2010): Experiences in Intercultural Training in Germany and India. A Comparison of the German and Indian Mind-set at the Workplace. In Casper-Hehne, Hiltraud, Gupte, Niteen and Stilz, Gerhard (eds.): Kommunikation über Grenzen: aktuelle Ansätze zur interkulturellen Verständigung Göttingen: Universitätsverlag Göttingen, 39-44.

Nassehi, Armin (2005): Organizations as decision machines: Niklas Luhmann's theory of organized social systems. The Sociological Review, 53, 178-91.

Nayak, Amar (2011): Indian multinationals: the dynamics of explosive growth in a developing country context. Basingstoke: Palgrave Macmillan.

Neuberger, Oswald and Kompa, Ain (1987): Wir, die Firma. Der Kult um die Unternehmenskultur. München: Heyne.

news.com.au (2016): Bosses are refusing to pay for work drinks, but it could be their loss, October 26th 2016. URL: http://www.news.com.au/financ e/small-business/bosses-are-refusing-to-pay-for-work-drinks-but-it-c ould-be-their-loss/news-story/bd76d769e13881897677441f9d47e297 [last accessed 19.05.2021]

Ogasawara, Yuko (1998): Office ladies and salaried men: Power, gender, and work in Japanese companies. Berkeley: University of California Press.

Orr, Julian (1996): Talking about machines: An ethnography of a modern job. London: Cornell University Press.

Ouchi, William and Wilkins, Alan (1985): Organizational Culture. Annual Review of Sociology, 11 (1), 457-83.

Panini, M. N. 2015. Embourgeoisement and the Middle Classes in India. In Lobo, Nancy and Shah, Jayesh (eds.): The trajectory of India's middle class : 
economy, ethics and etiquette, Newcastle upon Tyne: Cambridge Scholars Publishing, 14-39.

Papinot, Christian (2007): Le 'malentendu productif': Réflexion sur la photographie comme support d'entretien. Ethnologie française, 37 (1), 79-86.

Parry, Jonathan (2009): Satanic Fields, Pleasant Mills: Work in an Indian Steel Plant, in Mollona, Massimiliano, De Neve, Geert, and Parry, Jonathan (eds.), Industrial Work and Life: An Anthropological Reader (Oxford: berg), 65-82.

Pereira, Vijay and Malik, Ashish (eds.) (2015): Investigating Cultural Aspects in Indian Organizations: Empirical Evidence. Heidelberg: Springer.

Petermann, Werner (2010): Anthropologie unserer Zeit. Wuppertal: Hammer.

Peters, Tom and Waterman, Robert (1982): In search of excellence. Lessons from Americans Best Running Companies. New York: Harper \& Row.

Pink, Sarah (ed.) (2006): Applications of anthropology: professional anthropology in the twenty-first century. New York: Berghahn Books.

Platt, Jennifer, Crothers, Charles, and Horgan, Mervyn (2013): Producing Ethnographies: Workplace Ethnographies in History. Journal of the History of the Behavioral Sciences, 49 (1), 45-62.

Porter, Michael (2001): The value chain and competitive advantage, in Barnes, David (ed.): Understanding business: Processes London: Routledge, 50-66.

Rajmanohar, T. P. (ed.) (2007): India Inc. going global: the M\&A way. Hyderabad: Icfai University Press.

Rastogi, Gaurav and Pradhan, Basab (2011): Offshore: how india got back on global business map. New Delhi: Viking.

Rauh, Andreas (2012): Die besondere Atmosphäre: ästhetische Feldforschungen. Bielefeld: transcript.

Reed, Isaac (2006): Social dramas, shipwrecks, and cockfights: conflict and complicity in social performance, in Alexander, Jeffrey, Giesen, Bernhard and Mast, Jason (eds.): Social performance: symbolic action, cultural pragmatics, and ritual Cambridge: Cambridge University Press, 146-67.

Reeves, Carla (2010): A difficult negotiation: fieldwork relations with gatekeepers. Qualitative Research, 10 (3), 315-31.

Roberts, David (1995): Reconstructing Theory: Gadamer, Habermas, Luhmann. Melbourne: Melbourne University Press.

Rosen, Evan (2007): The Culture of Collaboration. Maximizing Time, Talend and Tools to create Value in the Global Economy. San Francisco: Red Ape. 
Roth, Hans-Joachim (2002): Kultur und Kommunikation: systematische und theoriegeschichtliche Umrisse Interkultureller Pädagogik. Wiesbaden: Springer.

Rottenburg, Richard (2009 [2002]): Far-fetched facts: a parable of development aid, translation by Allison Brown and Tom Lampert. London: MIT Press.

Røyrvik, Emil (2013): The allure of capitalism: an ethnography of management and the global economy in crisis. New York, Oxford: Berghahn Books.

Sahlins, Marshall (1982): The Apotheosis of Captain Cook, in Izard, Michel and Smith, Pierre (eds.): Between belief and transgression: structuralist essays in religion, history, and myth Chicago: University of Chicago Press, 73-102.

Salzinger, Leslie (2009) Gendered Meanings in Contention: Anarchomex, in Mollona, Massimiliano, De Neve, Geert, and Parry, Jonathan (eds.), Industrial Work and Life: An Anthropological Reader Oxford: Berg, 221-38.

Sandler, Jen and Thedvall, Renita (2017): Meeting Ethnography. Meetings as Key Technologies of Contemporary Governance, Development, and Resistance. New York: Routledge.

Scheffer, Thomas (2010): Ethnographie mit System am Beispiel von Englischen Strafverfahren, in John, René, Henkel, Anna, and Rückert-John, Jana (eds.): Die Methodologien des Systems: Wie kommt man zum Fall und wie dahinter? Wiesbaden: VS Verlag für Sozialwissenschaften, 141-60.

Schleiermacher, Friedrich and Bowie, Andrew (1998 [1838]): Schleiermacher: Hermeneutics and criticism: and other writings. Cambridge: Cambridge University Press.

Schnegg, Michael and Lang, Hartmut (2002): Netzwerkanalyse - Eine praxisorientierte Einführung. Methoden der Ethnographie, 1 (1.3), 2-54.

Schurz, Robert (1995): Negative Hermeneutik: zur sozialen Anthropologie des Nicht-Verstehens. Opladen: Westdeutscher Verlag.

Schwartzman, Helen (1989): The meeting: gatherings in organizations and communities. New York: Plenum Press.

Schwartzman, Helen (1993): Ethnography in organizations. Newbury Park: Sage.

Seidl, David (2005): The Basic Concepts of Luhmann's theory of social systems, in Seidl, David and Becker, Kai (eds.): Niklas Luhmann and organization studies Copenhagen: Liber Malmö, 21-53. 
Seitz, Tim (2017): Design Thinking und der neue Geist des Kapitalismus. Soziologische Betrachtungen einer Innovationskultur (29). Bielefeld: transcript.

Servais, Christine and Servais, Véronique (2009): Le malentendu comme structure de la communication. Questions de communication 15, 21-49.

Shah, Chirag (2014): Collaborative information seeking. Journal of the Association for Information Science and Technology, 65 (2), 215-36.

Shah, Grishma (2015): Zippies and the Shift in Cultural Values in India, in Pereira, Vijay and Malik, Ashish (eds.): Investigating Cultural Aspects in Indian Organizations: Empirical Evidence Heidelberg: Springer, 31-56.

Shore, Cris (2000): Building Europe: the cultural politics of European integration. London: Routledge.

Silverman, David (2007): A very short, fairly interesting and reasonably cheap book about qualitative research. Los Angeles: Sage.

Simon, Andrea (2016): How Corporate Anthropology Can Help Women Drive Change. Forbes online, March 28th 2016. URL: http://www.forbes.com/si tes/womensmedia/2016/03/28/how-corporate-anthropology-can-help-w omen-drive-change/print/ [last accessed 19.05.2021]

Singh, Vir (1979): Multinational corporations and India. New Delhi: Sterling Publishers.

Sinha, Jai and Sinha, D. (1990): Role of social values in Indian organizations. International Journal of Psychology, 25 (3-6), 705-14.

Smith, Vicki (2001): Ethnographies of Work and the Work of Ethnographers, in Atkinson, Paul (ed.): Handbook of ethnography Los Angeles: Sage, 22033.

Spear, Thomas (2003): Neo-Traditionalism and the Limits of Invention in British Colonial Africa. The Journal of African History, 44 (1), 3-27.

Sprenger, Guido (2016): Structured and unstructured misunderstandings. Towards an anthropological theory of misunderstanding. Civilisations, 64 (1\&2), 23-40.

Sprenger, Guido (2017): Communicated into being: Systems theory and the shifting of ontological status. Anthropological Theory, 17 (1), 108-132.

Spyridakis, Manos (2016): The liminal worker: An ethnography of work, unemployment and precariousness in contemporary Greece. London: Routledge.

Squires, Susan and Van De Vanter, Michael L. (2013): Communities of Practice, in Caulkins, Douglas and Jordan, Ann (eds.): A Companion to Organizational Anthropology Oxford: Wiley, 289-310. 
Sridhar, Devi (ed.) (2008): Anthropologists inside Organisations: South Asian case studies. Los Angeles: Sage.

Srinivasan, Vasanthi, Murty, L.S., and Nakra, Monisha (2013): Career persistence of women software professionals in India. Gender in Management, 28 (4), 210-27.

Steenkamp, Jan-Benedict and Geyskens, Inge (2012): Transaction cost economics and the roles of national culture: a test of hypotheses based on Inglehart and Hofstede. Journal of the Academy of Marketing Science, 40 (2), 252-70.

Stein, Felix (2017): Work, Sleep, Repeat. The Abstract Labour if German Management Consultants. London: Bloomsbury.

Stevenson, William, Bartunek, Jean, and Borgatti, Stephen (2003): Front and backstage processes of an organizational restructuring effort. The Journal of Applied Behavioral Science, 39 (3), 243-58.

Stodulka, Thomas (2014): Emotion Work, Ethnography, and Survival Strategies on the Streets of Yogyakarta. Medical Anthropology, 34 (1), 84-97.

Strauss, Anselm (1993): Continual permutations of action. New York: de Gruyter.

Strümpell, Christian (2006): 'Wir arbeiten zusammen, wir essen zusammen': Konvivium und soziale Peripherie in einer indischen Werkssiedlung. Münster: Lit.

Sturdy, Andrew, Schwarz, Mirela, and Spicer, Andre (2006): Guess who's coming to dinner? Structures and uses of liminality in strategic management consultancy. Human Relations, 59 (7), 929-60.

Sunderland, Patricia and Denny, Rita (2007): Doing anthropology in consumer research. Walnut Creek: Left Coast Press.

Taras, Vas, Steel, Piers, and Kirkman, Bradley (2016): Does Country Equate with Culture? Beyond Geography in the Search for Cultural Boundaries. Management International Review, 56 (4), 455-87.

Tempest, Sue and Starkey, Ken (2004): The Effects of Liminality on Individual and Organizational Learning. Organization Studies, 25 (4), 507-27.

Tian, Robert, Lillis, Michael, and Van Marrewijk, Alfons (2010): General Business Anthropology. Atlanta: North American Business Press.

Tilak, Jandhyala (2013): Higher education in India: in search of equality, quality and quantity. New Delhi: Orient Blackswan.

Tsing, Anna (2005) Friction: an ethnography of global connection. Princeton: Princeton University Press. 
Turner, Victor (1964): Betwixt and Between: The Liminal Period in Rites de Passage. The Proceedings of the American Ethnological Society (1964), Symposium on New Approaches to the Study of Religion, 4-20.

Uhrich, Sebastian (2008): Das Konstrukt Atmosphäre im Fußballstadion Konzeptionalisierung und Operationalisierung, in Benkenstein, Martin (ed.): Neue Herausforderungen an das Dienstleistungsmarketing Wiesbaden: Gabler, 41-77.

Upadhya, Carol (2016): Reengineering India: work, capital, and class in an offshore economy. New Delhi: Oxford University Press.

Upadhya, Carol and Vasavi, Ar (eds.) (2008): In an outpost of the global economy: work and workers in India's information technology industry. Delhi: Routledge.

Valtonen, Anu, Markuksela, Vesa, and Moisander, Johanna (2010): Doing sensory ethnography in consumer research. International Journal of Consumer Studies, 34 (4), 375-80.

Vedpuriswar, A. V. (2008): Globalization of Indian companies: concepts and cases. Hyderabad: ICFAI University Press.

Venaik, Sunil and Brewer, Paul (2016): National culture dimensions: The perpetuation of cultural ignorance. Management Learning, 47 (5), 563-89.

von Neumann, John and Morgenstern, Oskar (2007): Theory of games and economic behavior. Princeton: Princeton University Press.

von Nordenflycht, Andrew (2010): What Is a Professional Service Firm? Toward a Theory and Taxonomy of Knowledge-Intensive Firms. The Academy of Management Review, 35 (1), 155-74.

Voswinkel, Stephan (2005) Welche Kundenorientierung? Anerkennung in der Dienstleistungsarbeit. Berlin: Sigma.

Watkins, Susan and Swidler, Ann (2013): Working Misunderstandings: Donors, Brokers, and Villagers in Africa's AIDS Industry. Population and Development Review, 38, 197-218.

Wels, Harry (2003): Private wildlife conservation in Zimbabwe: Joint ventures and reciprocity. Leiden: Brill.

Wijsen, Frans and Tanner, Ralph (2008): The Limitations of an Ecumenical Language: The Case of Ki-Swahili. Anthropos, 103 (2), 549-54.

Williams, Amanda and Irani, Lilly (2010): There's methodology in the madness: toward critical HCI ethnography. CHI'10 Extended Abstracts on Human Factors in Computing Systems (ACM), 2725-34.

Winkler, Ingo and Mahmood, Mustafa (2015): The Liminality of Temporary Agency Work: Exploring the Dimensions of Danish Temporary Agency 
Workers' Liminal Experience. Nordic journal of working life studies, 5 (1), 51-68.

Winkler, Jessica, Dibbern, Jens, and Heinzl, Armin (2007): Der einfluss kultureller unterschiede beim IT-offshoring. Wirtschaftsinformatik, 49 (2), 95-103.

Wittel, Andreas (1997): Belegschaftskultur im Schatten der Firmenideologie: eine ethnographische Fallstudie. Berlin: Sigma.

Wolcott, Harry F. (2003 [1973]): The man in the principal's office. An ethnography. Walnut Creek: AltaMira Press.

Wright, Susan (1994): 'Culture' in anthropology and organizational studies, in Wright, Susan (ed.): Anthropology of organizations, London: Routledge, 1-31.

Ybema, Sierk, Yanow, Dvora, Wels, Harry, and Kamsteeg, Frans (2009): Studying everyday organizational life, in Ybema, Sierk, Yanow, Dvora, Wels, Harry, and Kamsteeg, Frans (eds.): Organizational ethnography: Studying the complexity of everyday life Los Angeles: Sage, 1-20. 


\section{Cultural Studies}

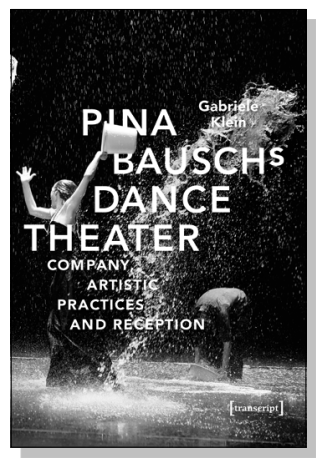

Gabriele Klein

Pina Bausch's Dance Theater

Company, Artistic Practices and Reception

2020, 440 p., pb., col. ill.

29,99€ (DE), 978-3-8376-5055-6

E-Book:

PDF: 29,99 € (DE), ISBN 978-3-8394-5055-0

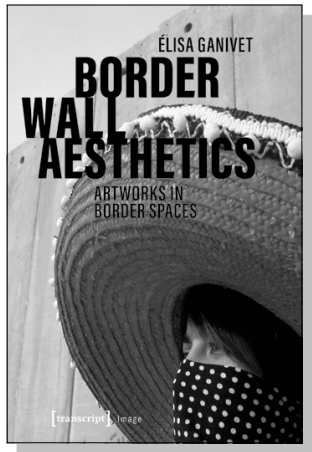

Elisa Ganivet

\section{Border Wall Aesthetics}

Artworks in Border Spaces

2019, 250 p., hardcover, ill.

79,99€ (DE), 978-3-8376-4777-8

E-Book:

PDF: 79,99€ (DE), ISBN 978-3-8394-4777-2

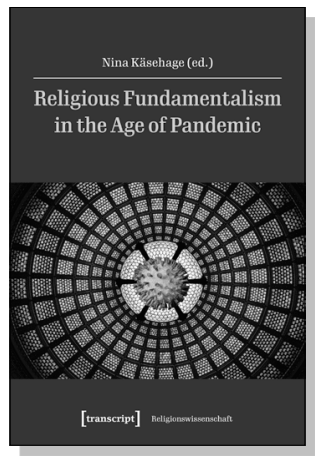

Nina Käsehage (ed.)

\section{Religious Fundamentalism} in the Age of Pandemic

April 2021, 278 p., pb., col. ill. 37,00 € (DE), 978-3-8376-5485-1

E-Book: available as free open access publication PDF: ISBN 978-3-8394-5485-5 


\section{Cultural Studies}
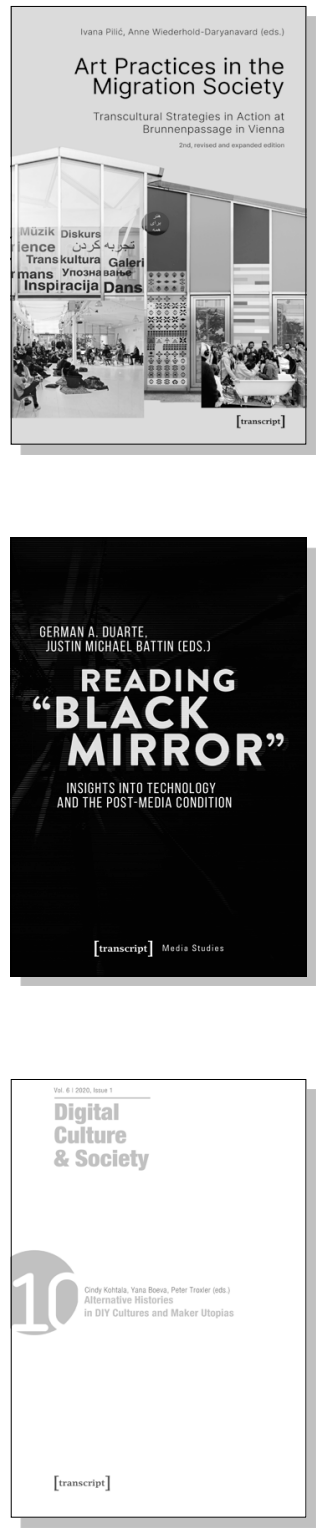

Ivana Pilic, Anne Wiederhold-Daryanavard (eds.) Art Practices in the Migration Society Transcultural Strategies in Action at Brunnenpassage in Vienna

March 2021, 244 p., pb.

29,00€ (DE), 978-3-8376-5620-6

E-Book:

PDF: 25,99€ (DE), ISBN 978-3-8394-5620-0

German A. Duarte, Justin Michael Battin (eds.)

\section{Reading "Black Mirror"}

Insights into Technology and the Post-Media Condition

January 2021, 334 p., pb.

32,00€ (DE), 978-3-8376-5232-1

E-Book:

PDF: 31,99 € (DE), ISBN 978-3-8394-5232-5

Cindy Kohtala, Yana Boeva, Peter Troxler (eds.)

\section{Digital Culture \& Society (DCS)}

Vol. 6, Issue 1/2020 -

Alternative Histories in DIY Cultures and Maker Utopias

February 2021, 214 p., pb., ill.

29,99€ (DE), 978-3-8376-4955-0

E-Book:

PDF: 29,99€ (DE), ISBN 978-3-8394-4955-4 
